\title{
Ru-Catalyzed Chemo- and Enantioselective Hydrogenation of 2,4-Pentadien-1-ones: Synthesis of Chiral 2,4-Pentadien-1-ols
}

Chengyang Li, ${ }^{\dagger}$ Wenkui Lu, ${ }^{\dagger}$ Bin Lu, ${ }^{\dagger}$ Wanfang Li, ${ }^{\dagger}$ Xiaomin Xie, ${ }^{\dagger}$ and Zhaoguo Zhang $*, \dagger$,

†Shanghai Key Laboratory for Molecular Engineering of Chiral Drugs, School of Chemistry and Chemical Engineering, Shanghai Jiao Tong University, 800 Dongchuan Road, Shanghai 200240, China

\$College of Science, University of Shanghai for Science and Technology, Shanghai 200093, China

${ }^{\S}$ Shanghai Institute of Organic Chemistry, Chinese Academy of Sciences, 345 Lingling Road, Shanghai 200032, China

zhaoguo@sjtu.edu.cn

\section{Support Information}

\section{Table of contents}

1. NMR Spectra. .S2

2. HPLC Data. $\mathrm{S} 90$ 
1. NMR Spectra.

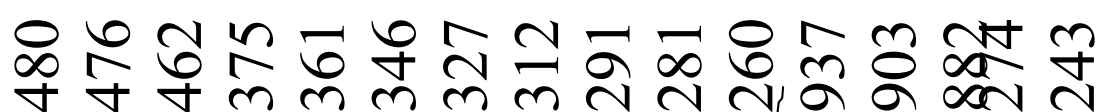

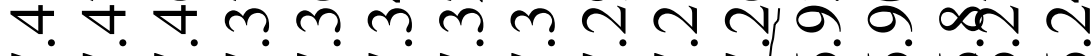
NNN N N N N
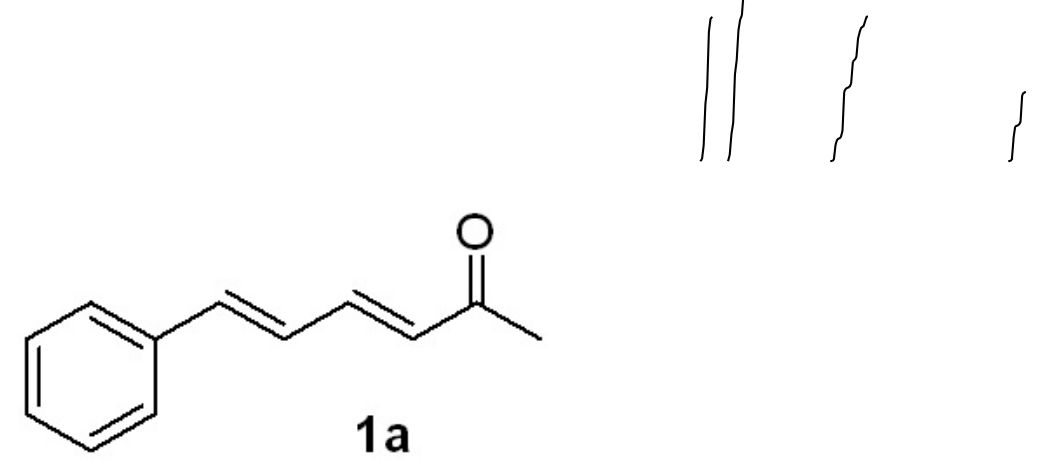

${ }^{1} \mathrm{H}$ NMR $\left(500 \mathrm{MHz}, \mathrm{CDCl}_{3}\right)$

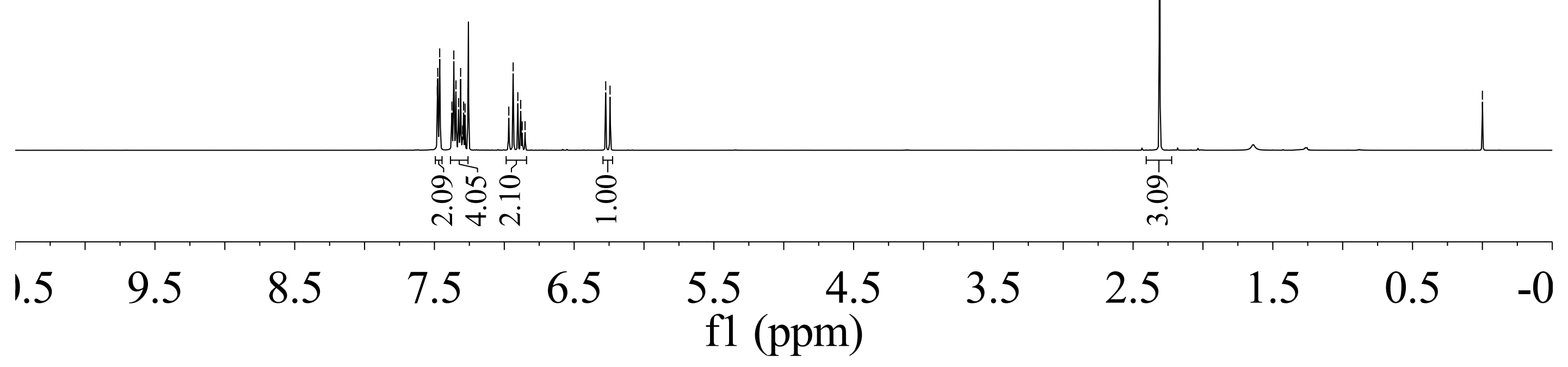



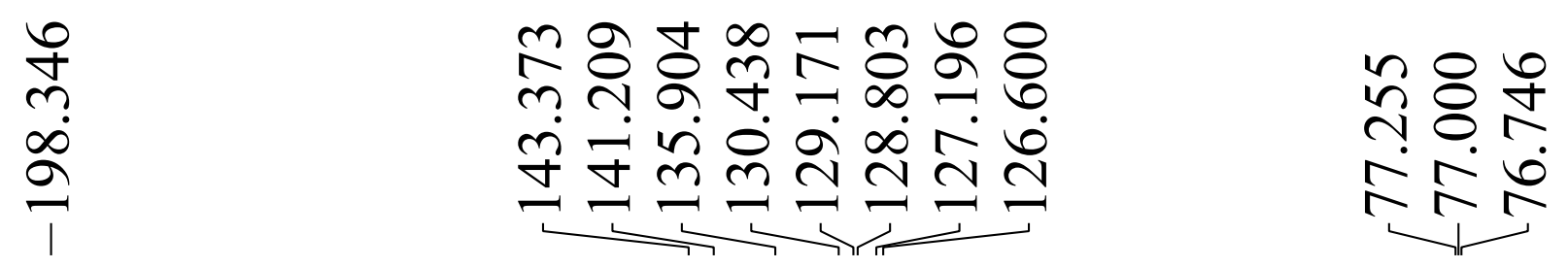

N

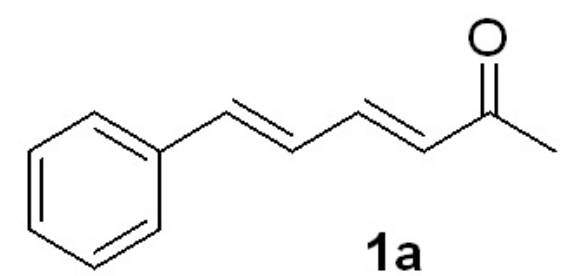

${ }^{13} \mathrm{C}$ NMR $\left(125 \mathrm{MHz}, \mathrm{CDCl}_{3}\right)$

$\begin{array}{rrrrrr}210 & 190 & 170 & 150 & 130 & 110 \quad 90 \\ & & & & & \end{array}$



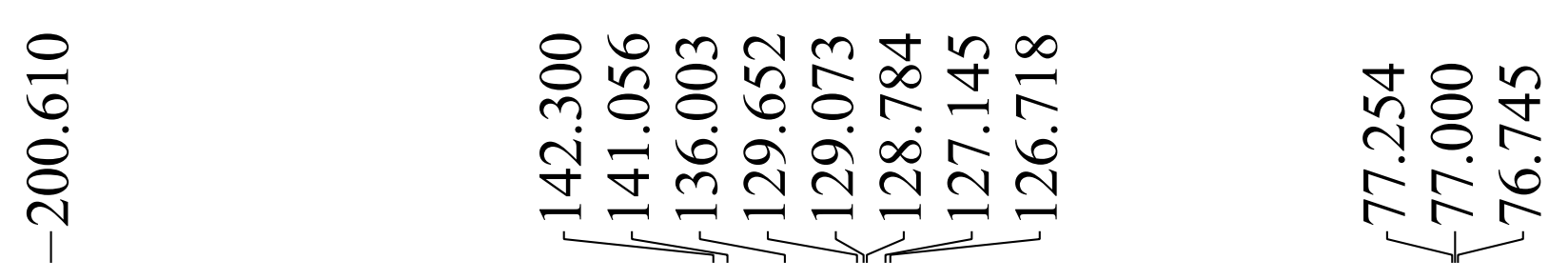

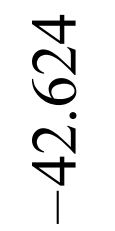

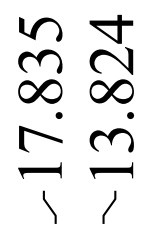

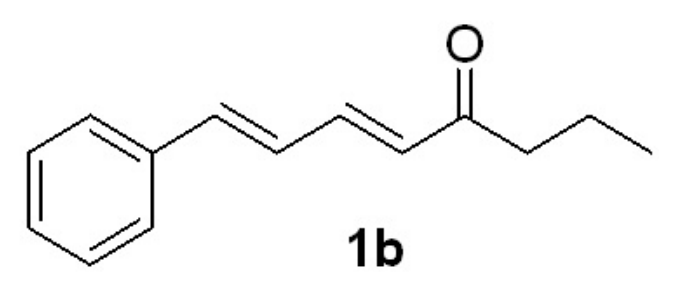

${ }^{13} \mathrm{C}$ NMR $\left(125 \mathrm{MHz}, \mathrm{CDCl}_{3}\right)$

\begin{tabular}{|c|c|c|c|c|c|c|c|c|c|c|}
\hline 210 & 190 & 170 & 150 & 130 & 110 & $\begin{array}{l}90 \\
\mathrm{~m})\end{array}$ & 70 & 50 & 30 & 10 \\
\hline
\end{tabular}




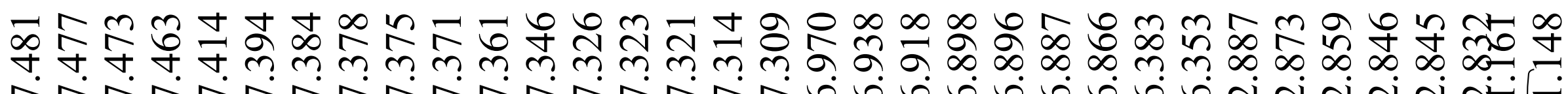

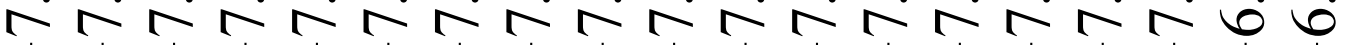

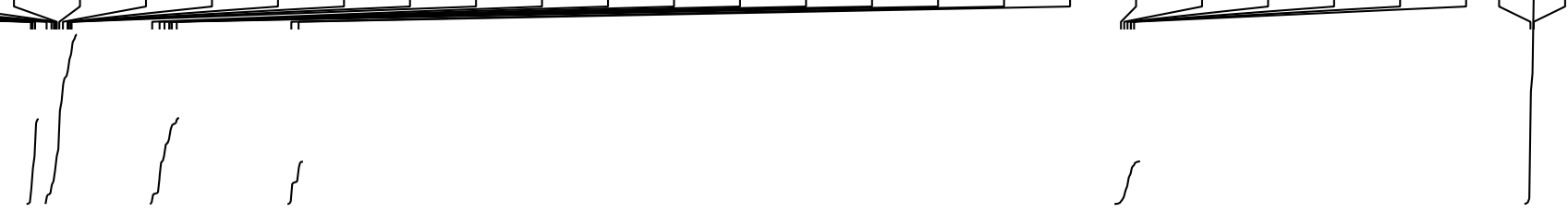

$\stackrel{8}{8}$

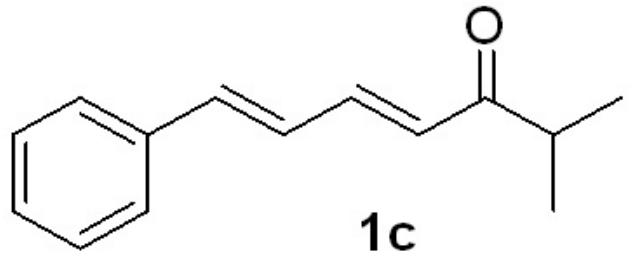

${ }^{1} \mathrm{H}$ NMR $\left(500 \mathrm{MHz}, \mathrm{CDCl}_{3}\right)$

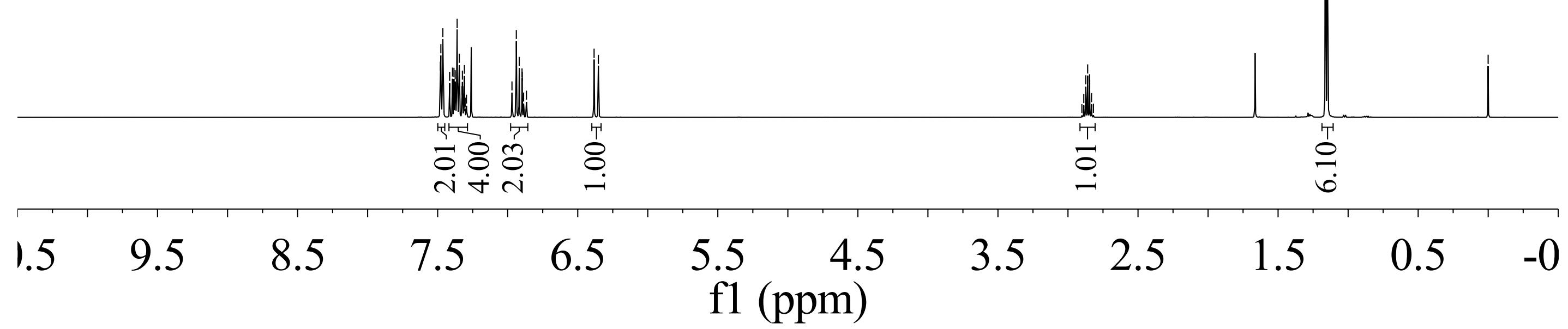




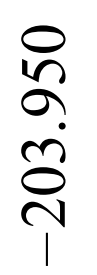
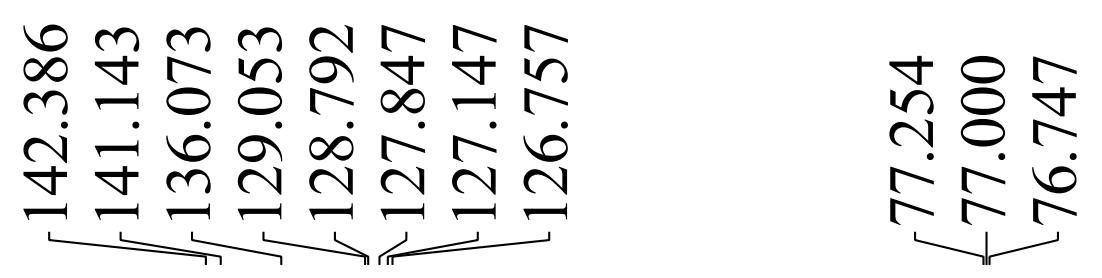

กิ่

\begin{tabular}{l}
$n$ \\
\multirow{1}{\infty}{} \\
1
\end{tabular}

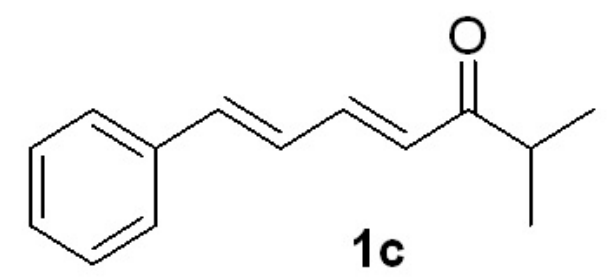

${ }^{13} \mathrm{C}$ NMR $\left(125 \mathrm{MHz}, \mathrm{CDCl}_{3}\right)$

\begin{tabular}{|c|c|c|c|c|}
\hline 210 & 190 & 170 & 150 & 130 \\
\hline
\end{tabular}




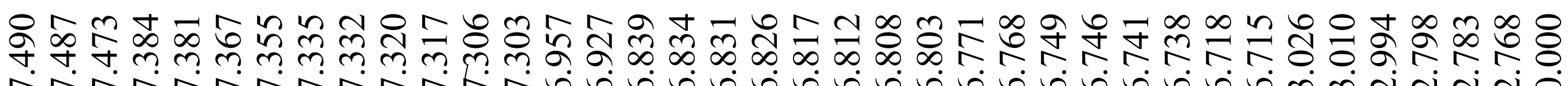

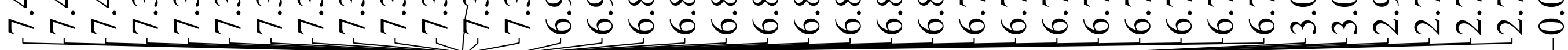
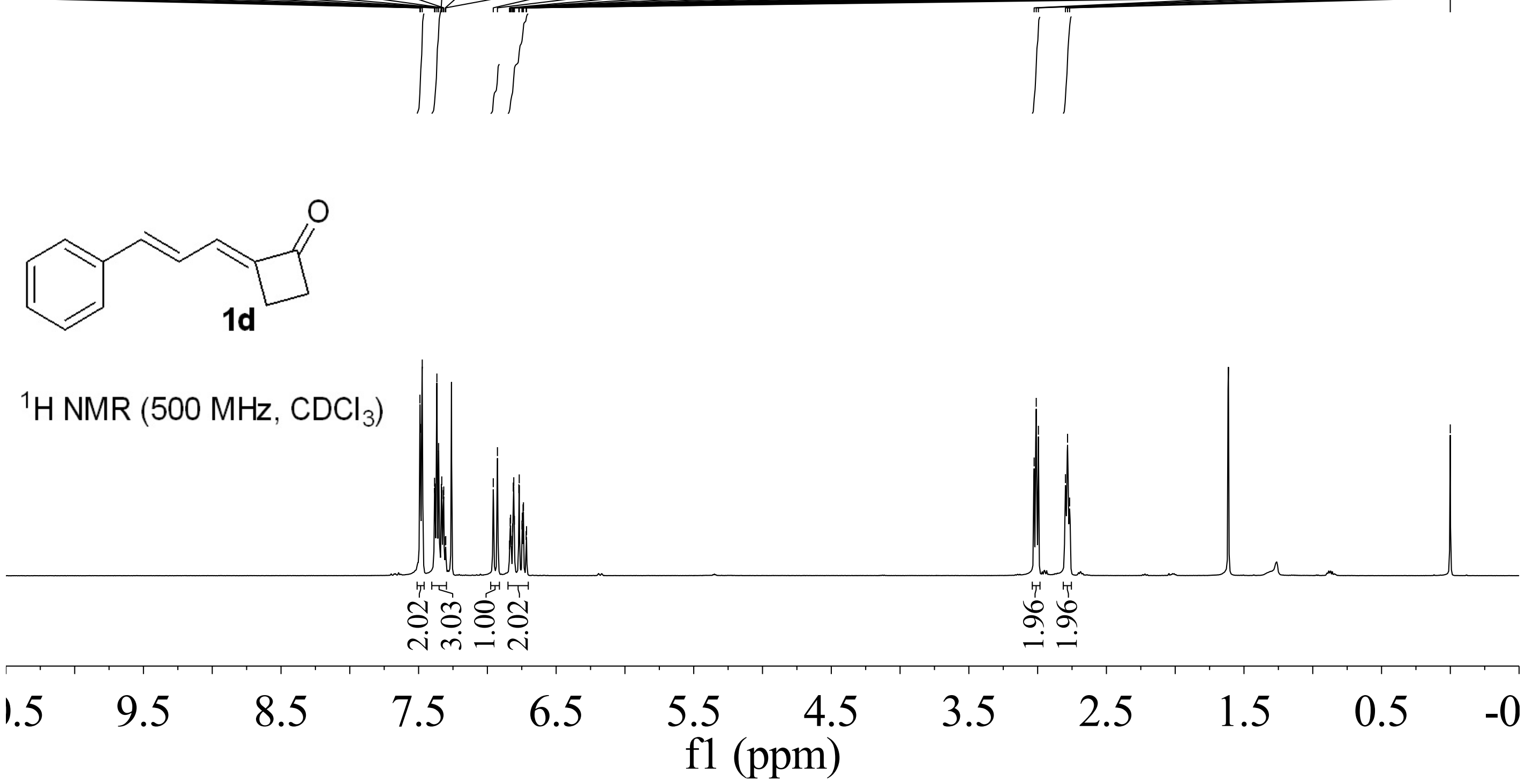

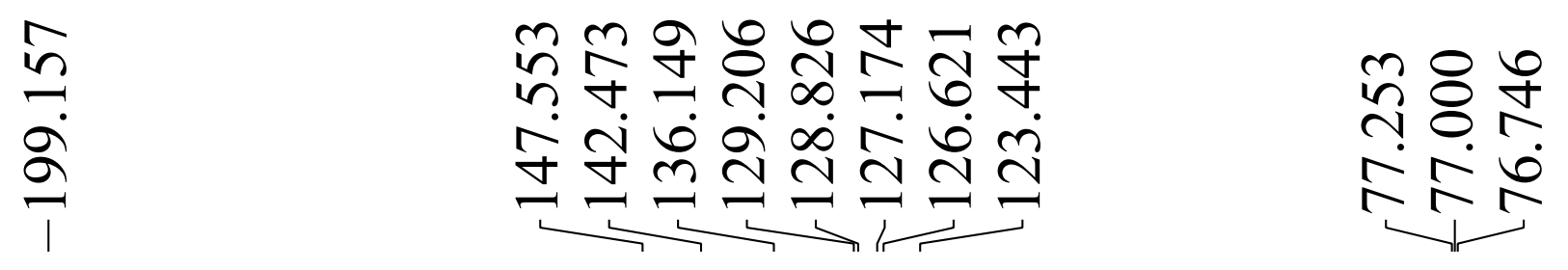

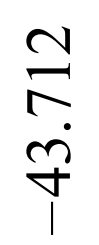

$\stackrel{ }{\stackrel{2}{ }}$

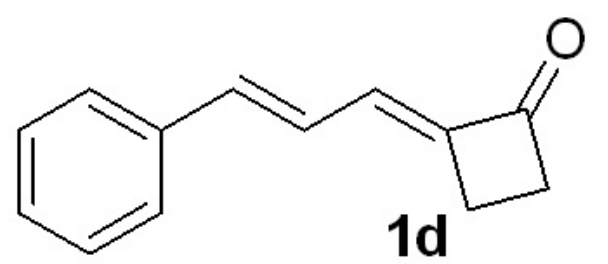

${ }^{13} \mathrm{C}$ NMR $\left(125 \mathrm{MHz}, \mathrm{CDCl}_{3}\right)$

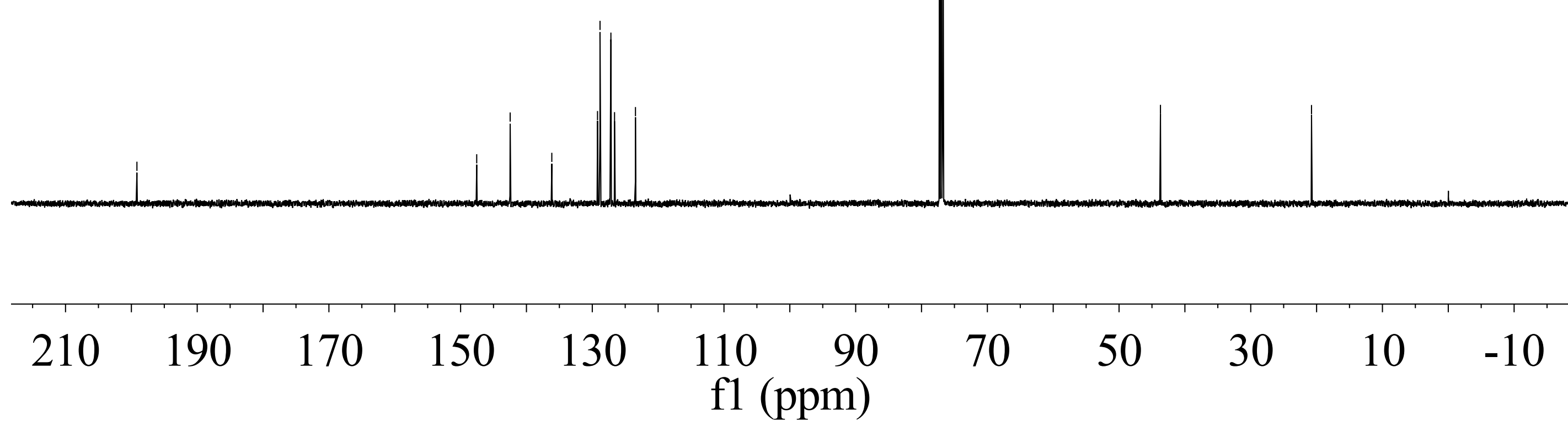




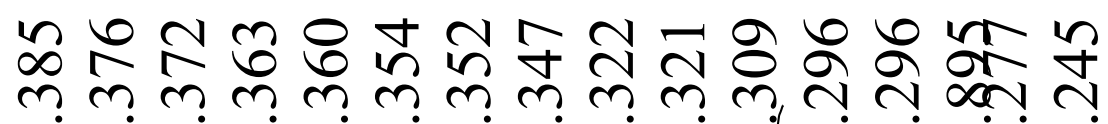

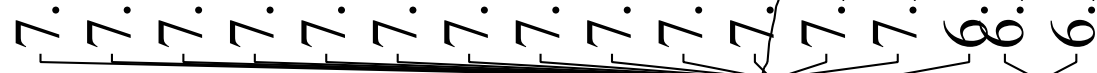

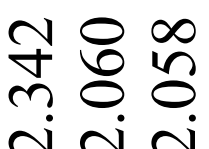

N

$\underset{8}{8}$

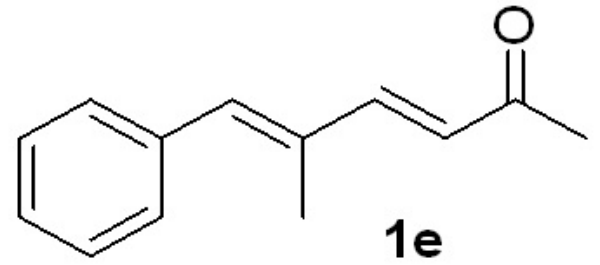

${ }^{1} \mathrm{H}$ NMR $\left(500 \mathrm{MHz}, \mathrm{CDCl}_{3}\right)$
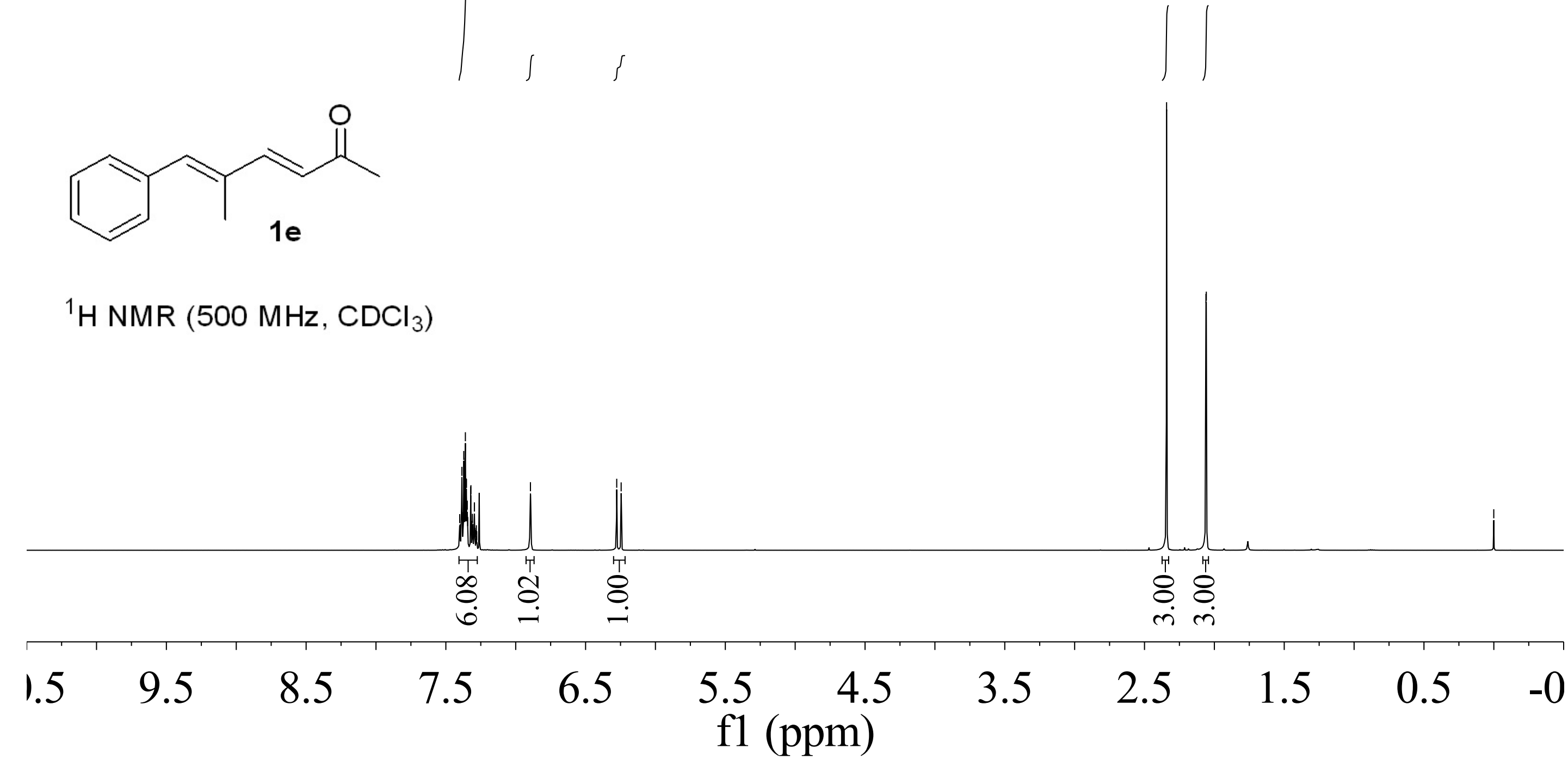

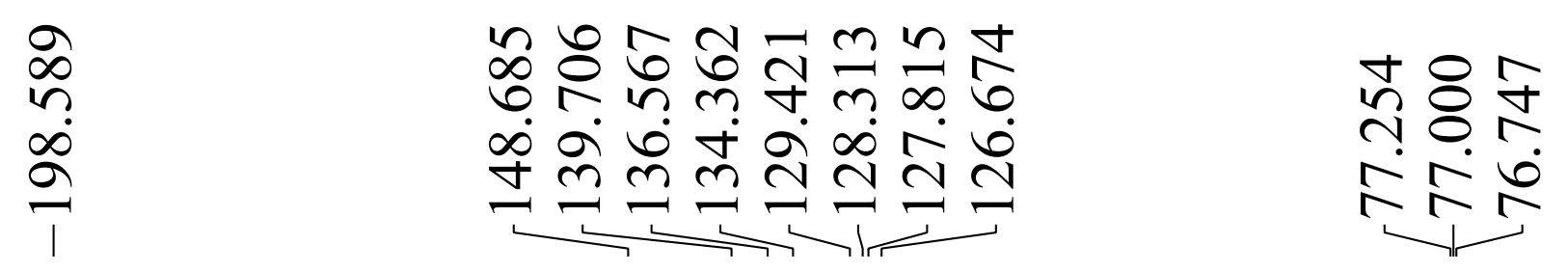

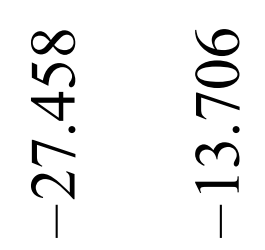

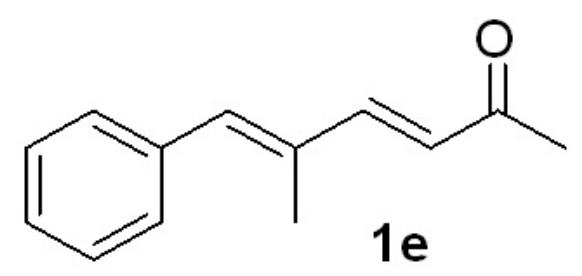

${ }^{13} \mathrm{C} \mathrm{NMR}\left(125 \mathrm{MHz}, \mathrm{CDCl}_{3}\right)$

\begin{tabular}{|c|c|c|c|c|c|c|c|c|c|c|}
\hline 210 & 190 & 170 & 150 & 130 & 110 & $\begin{array}{c}90 \\
\mathrm{~m})\end{array}$ & 70 & 50 & 30 & -10 \\
\hline
\end{tabular}


๗ָำ

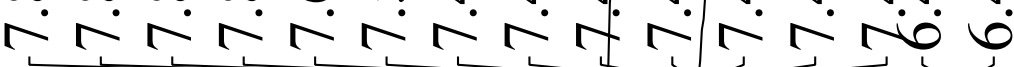

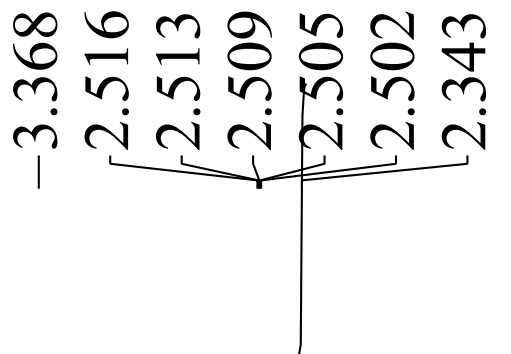

8
8
0
1

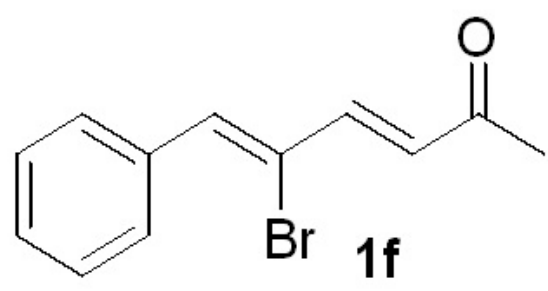

${ }^{1} \mathrm{H}$ NMR (500 MHz, d6-DMSO)

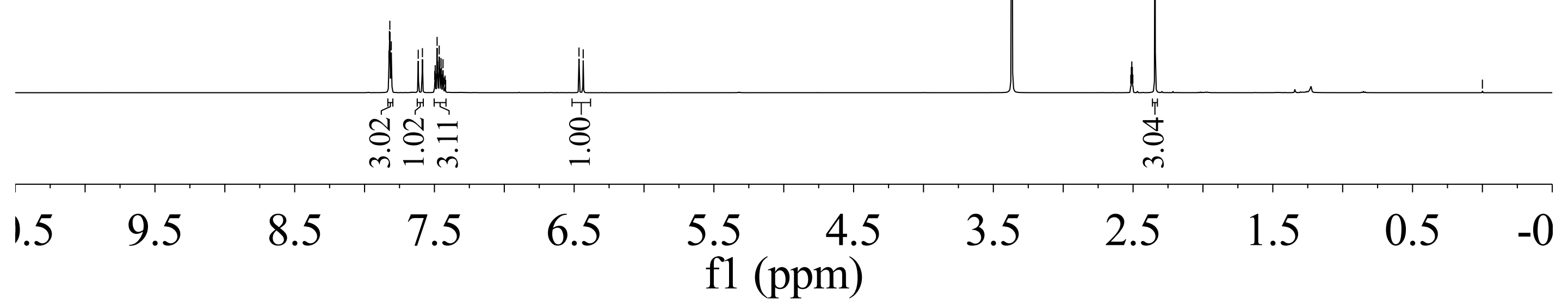



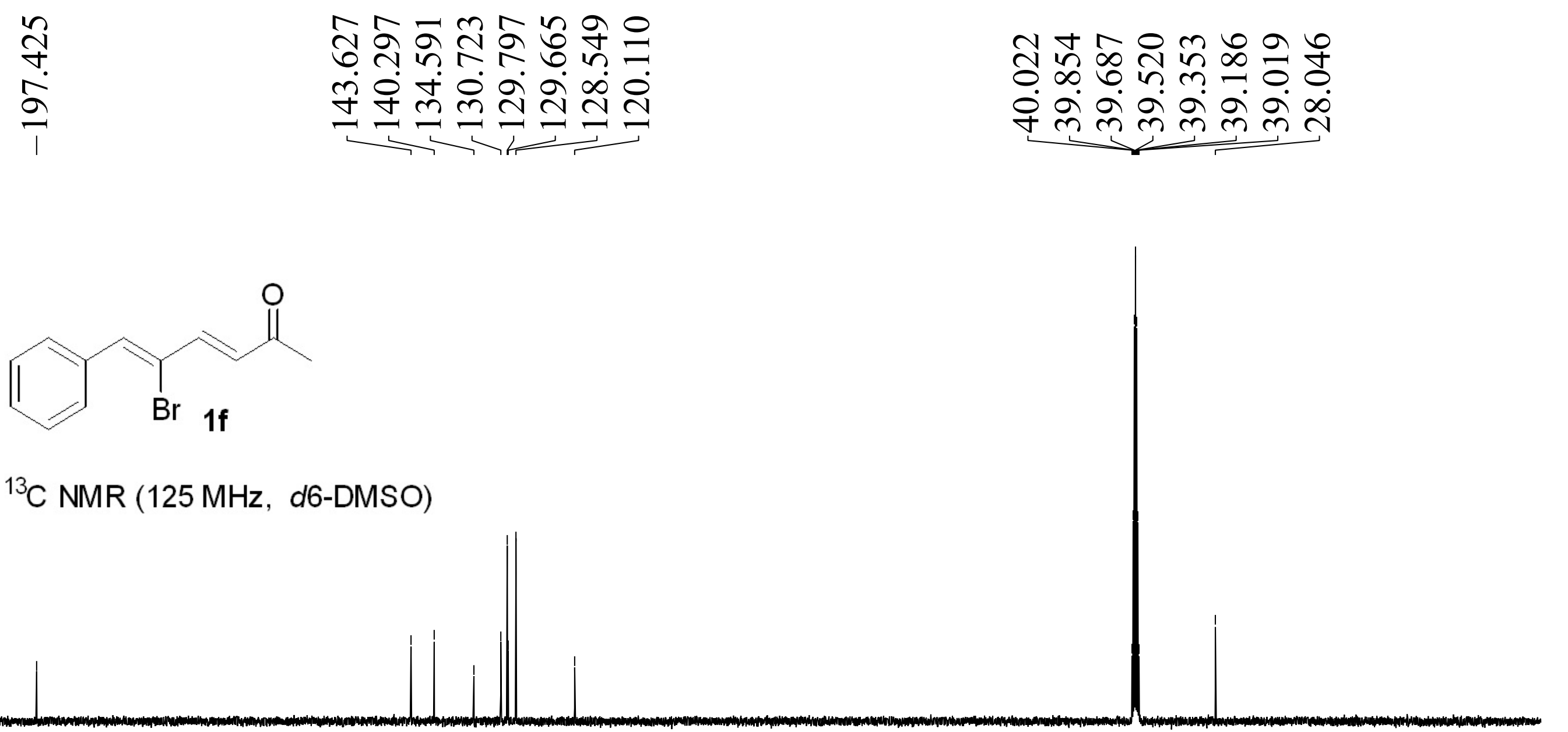

${ }^{13} \mathrm{C}$ NMR (125 MHz, d6-DMSO)

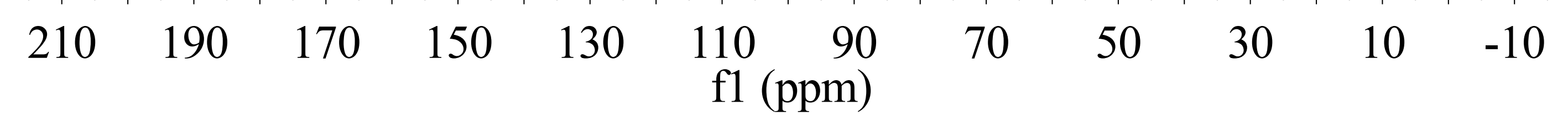




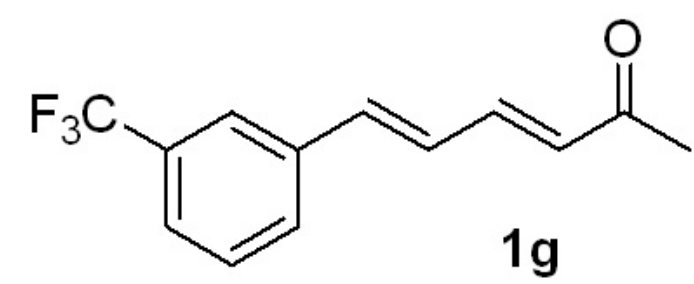

${ }^{1} \mathrm{H}$ NMR $\left(500 \mathrm{MHz}, \mathrm{CDCl}_{3}\right)$

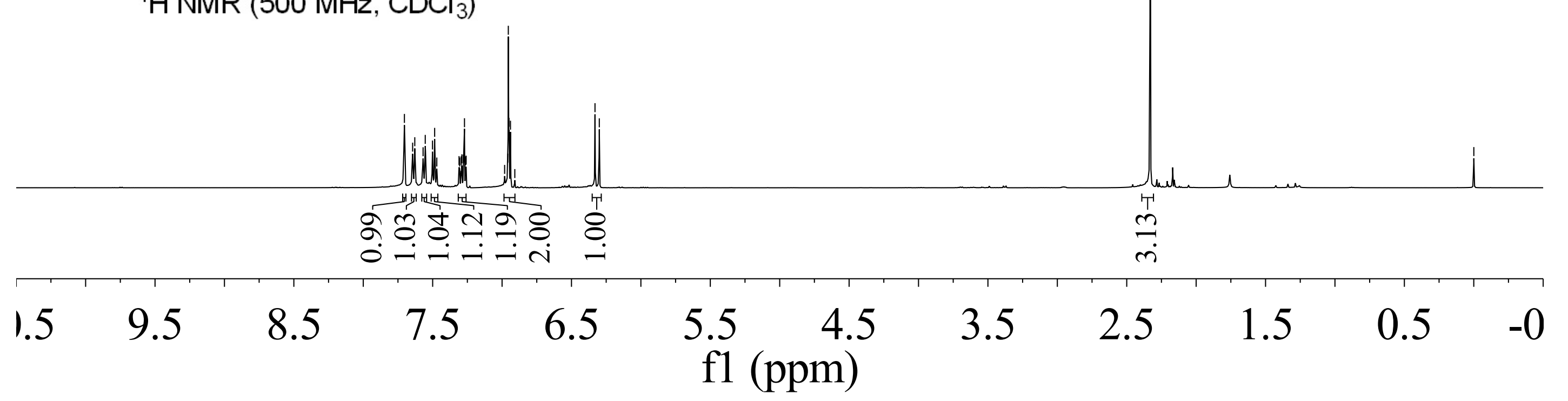




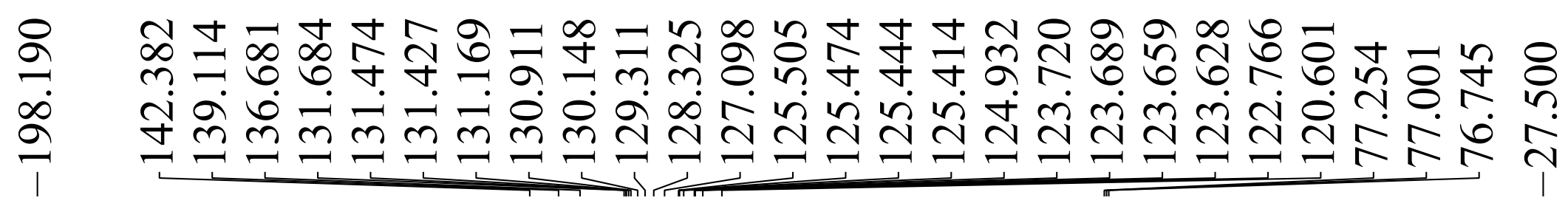

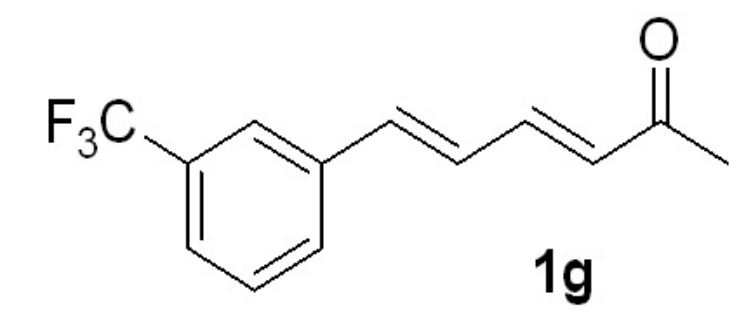

${ }^{13} \mathrm{C} \mathrm{NMR}\left(125 \mathrm{MHz}, \mathrm{CDCl}_{3}\right)$

$\begin{array}{llllll}210 & 190 & 170 & 150 & 130 & 110 \quad 9 \\ \mathrm{fl}(\mathrm{ppm})\end{array}$




$$
\begin{aligned}
& 0 \\
& \infty \\
& \multirow{1}{*}{} \\
& 1
\end{aligned}
$$

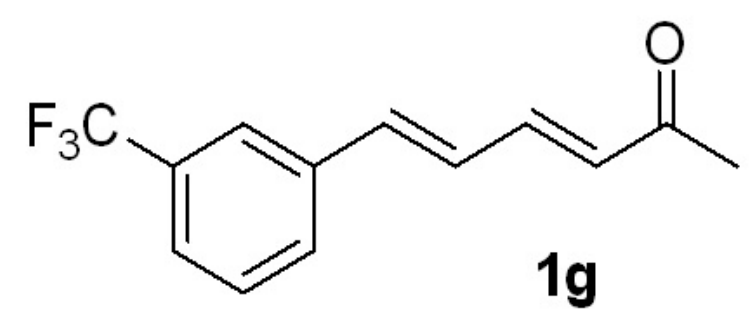

${ }^{19} \mathrm{~F} \mathrm{NMR}\left(471 \mathrm{MHz}, \mathrm{CDCl}_{3}\right)$

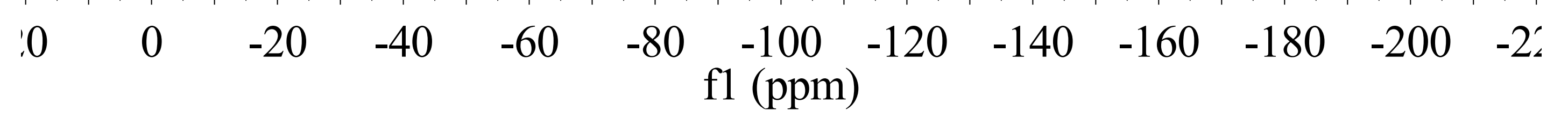




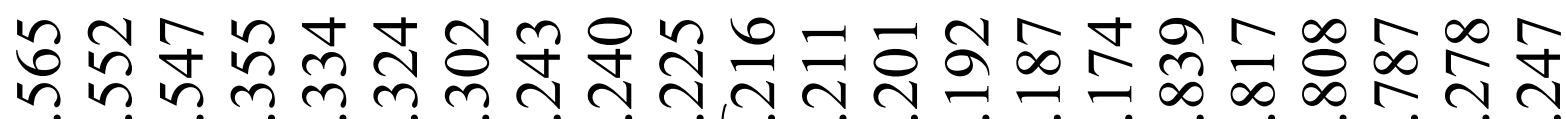

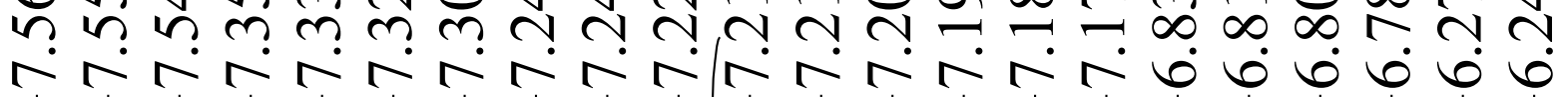

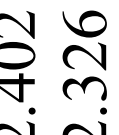

$\oint_{1}^{8}$

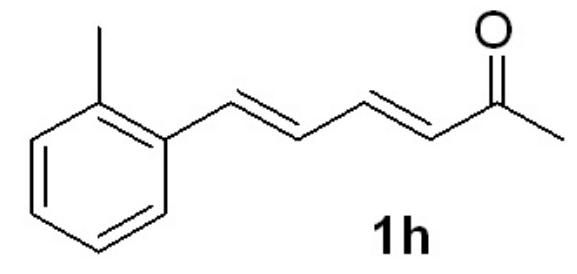

${ }^{1} \mathrm{H} \mathrm{NMR}\left(500 \mathrm{MHz}, \mathrm{CDCl}_{3}\right)$

N
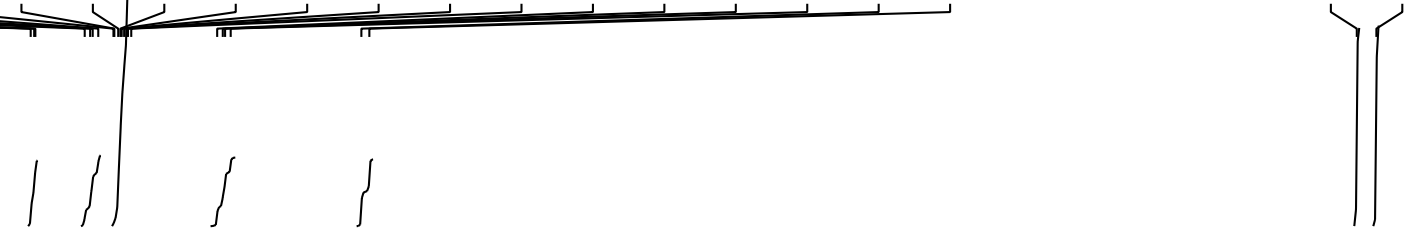


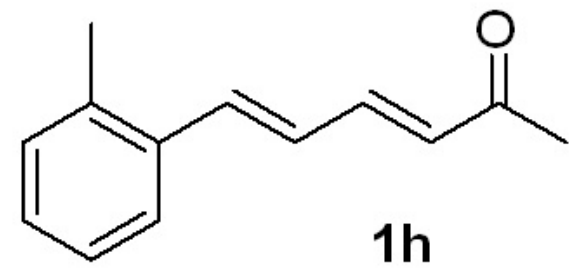

${ }^{13} \mathrm{C}$ NMR $\left(125 \mathrm{MHz}, \mathrm{CDCl}_{3}\right)$

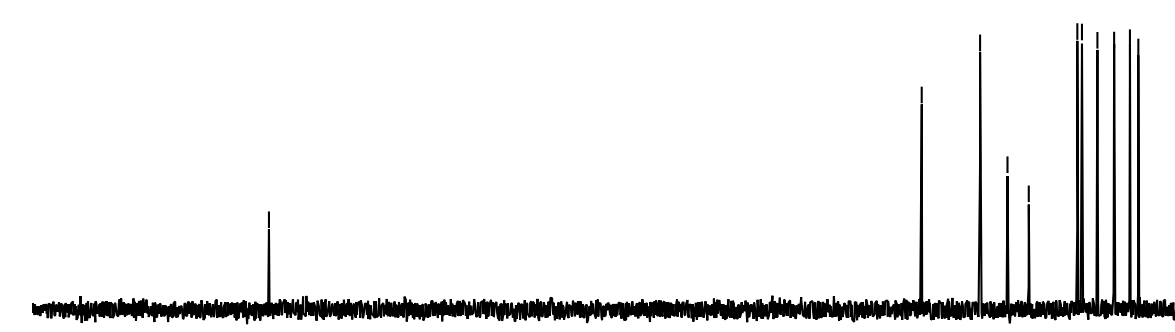

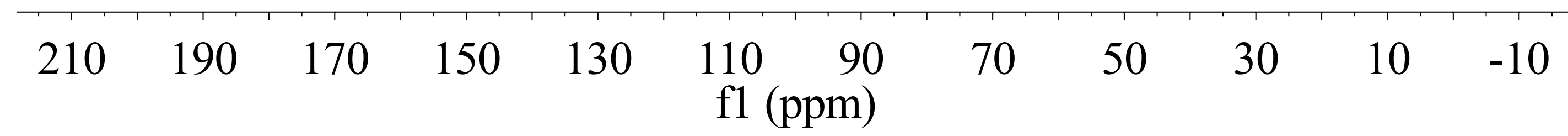




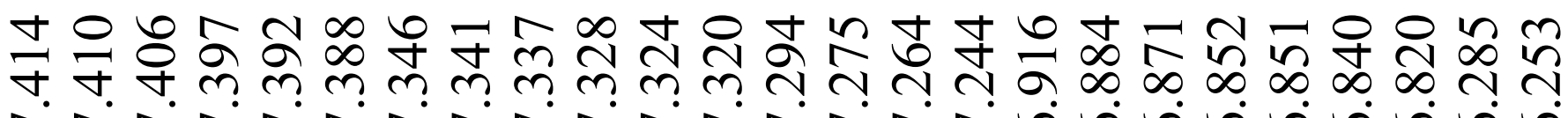

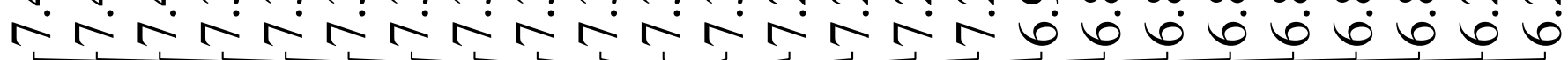

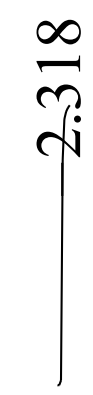

$\underset{\wp}{8}$

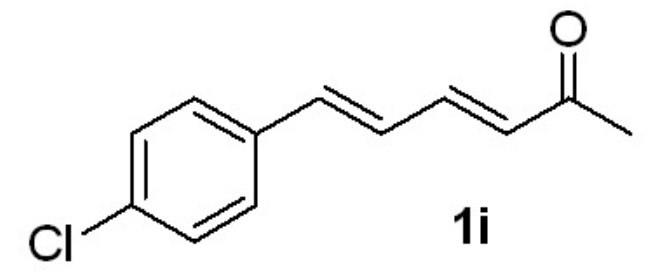

${ }^{1} \mathrm{H}$ NMR $\left(500 \mathrm{MHz}, \mathrm{CDCl}_{3}\right)$

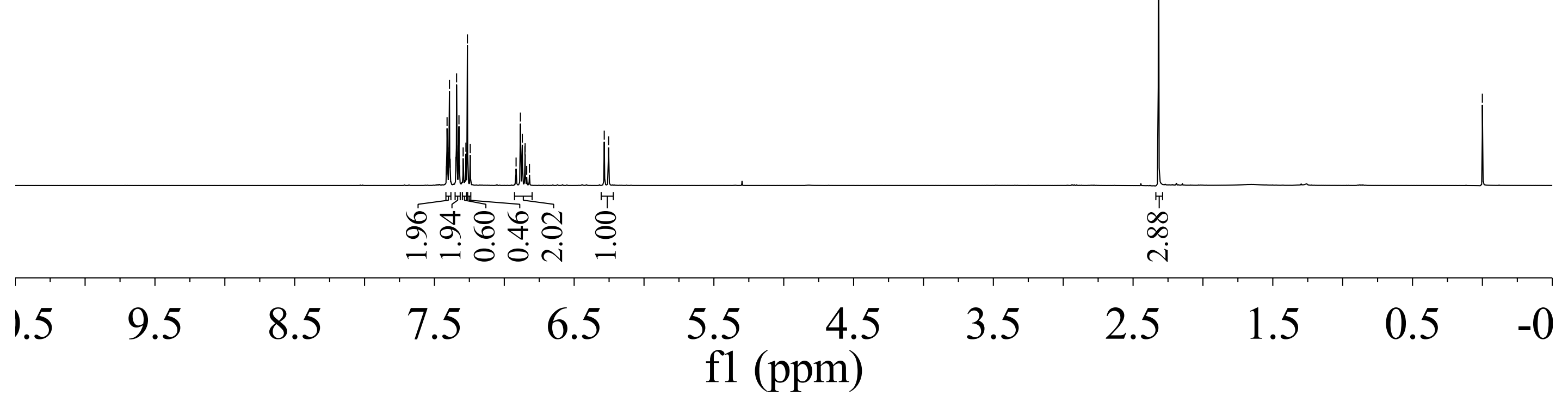



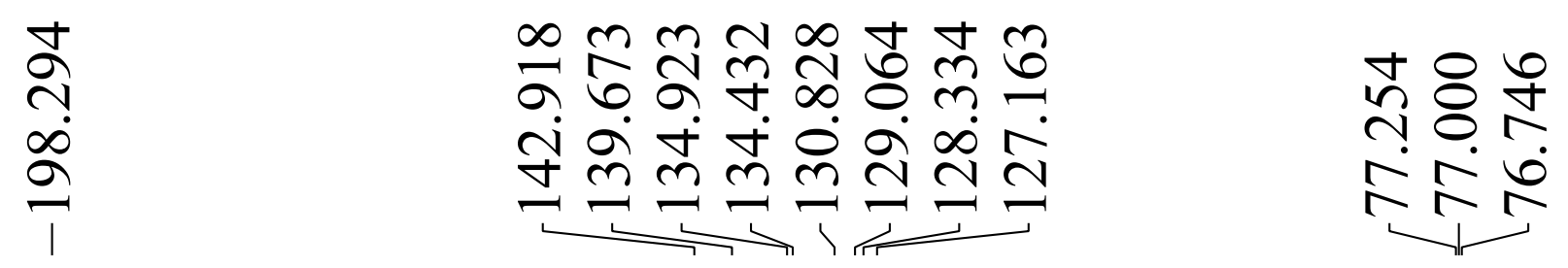

$\frac{n}{7}$

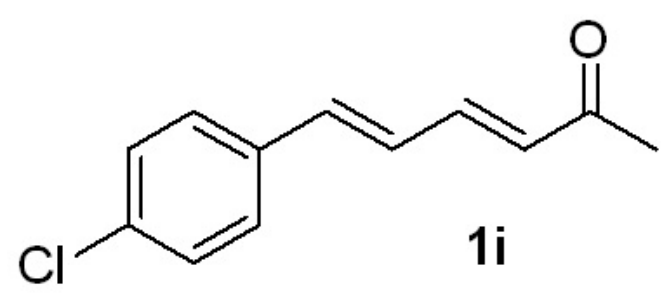

${ }^{13} \mathrm{C}$ NMR $\left(125 \mathrm{MHz}, \mathrm{CDCl}_{3}\right)$

$\begin{array}{rrrrrrr}210 & 190 & 170 & 150 & 130 & 110 \quad \begin{array}{r}90 \\ \text { f1 (ppm) }\end{array}\end{array}$

$\begin{array}{lllll}70 & 50 & 30 & 10 & -10\end{array}$




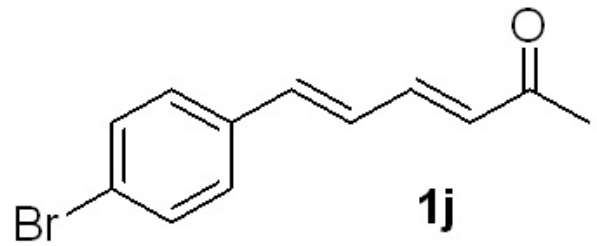

${ }^{1} \mathrm{H} \mathrm{NMR}\left(500 \mathrm{MHz}, \mathrm{CDCl}_{3}\right)$

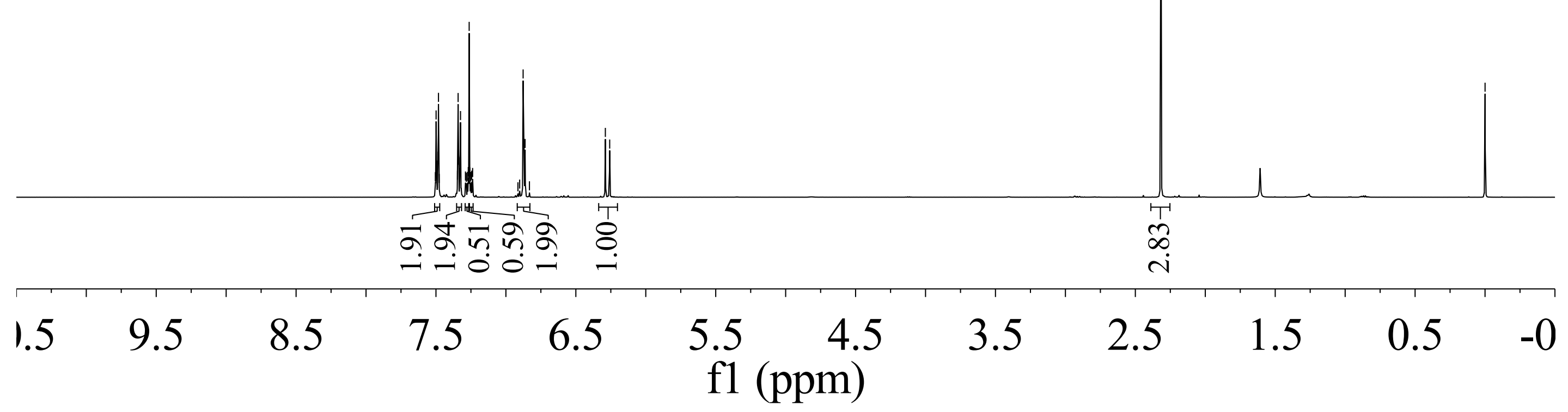



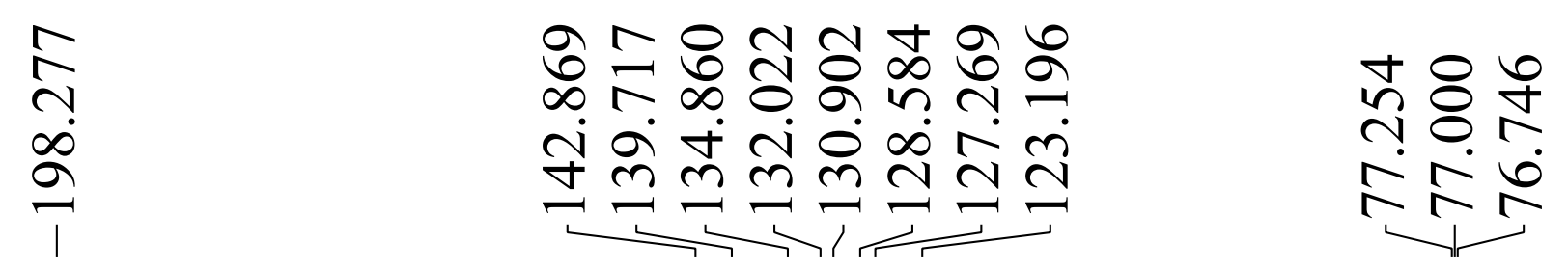

ণั

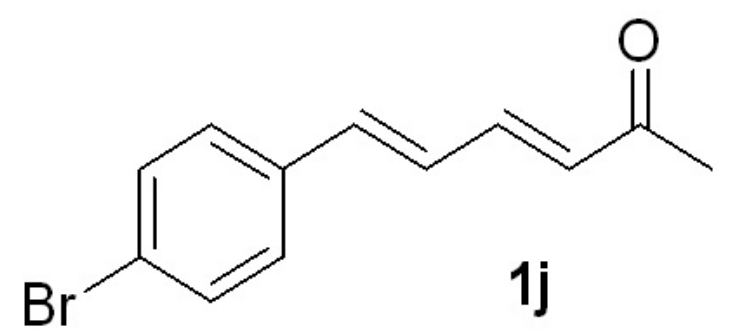

${ }^{13} \mathrm{C}$ NMR $\left(125 \mathrm{MHz}, \mathrm{CDCl}_{3}\right)$

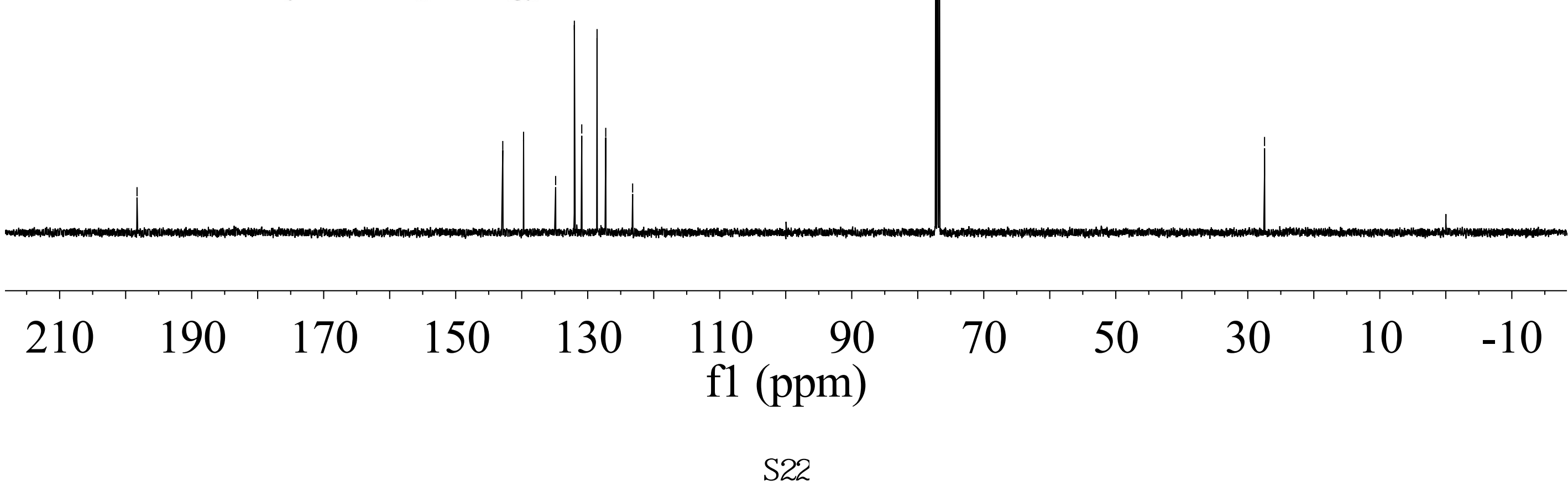




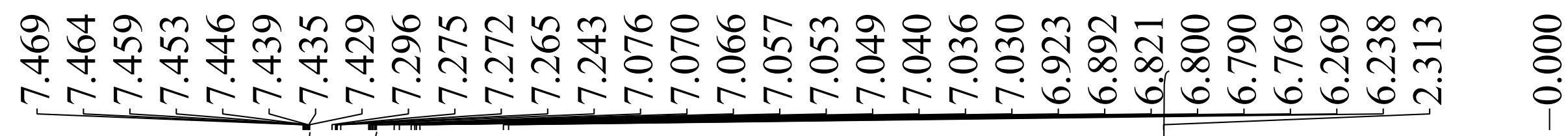

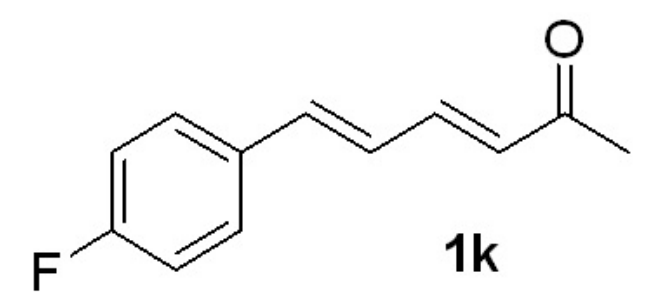

${ }^{1} \mathrm{H}$ NMR $\left(500 \mathrm{MHz}, \mathrm{CDCl}_{3}\right)$

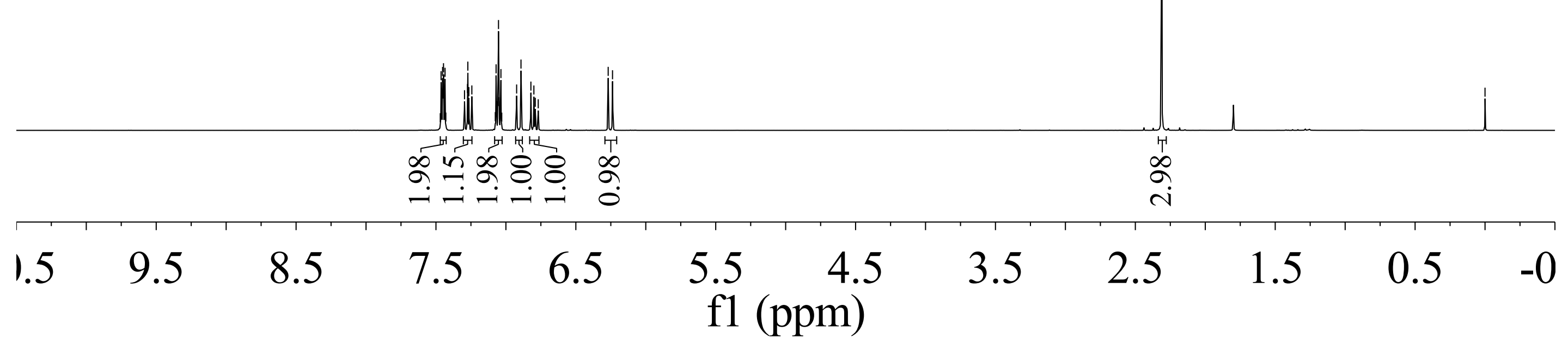




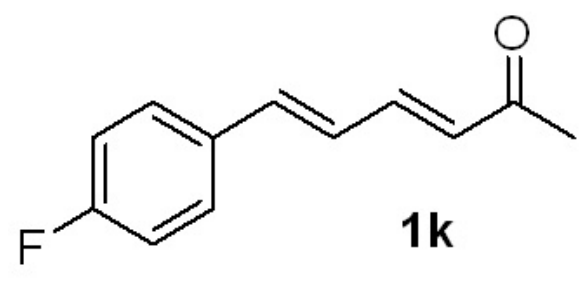

${ }^{13} \mathrm{C} \mathrm{NMR}\left(125 \mathrm{MHz}, \mathrm{CDCl}_{3}\right)$

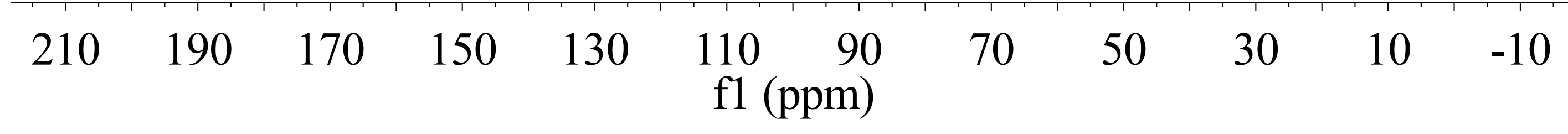




$$
\stackrel{9}{=}
$$

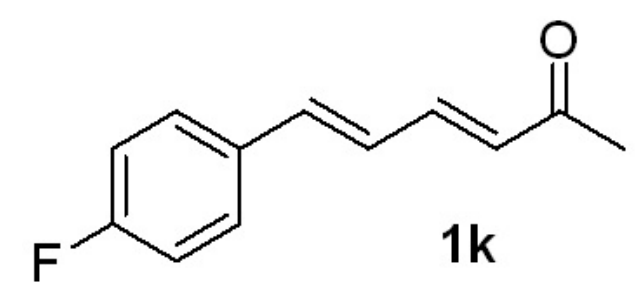

${ }^{19} \mathrm{~F} \mathrm{NMR}\left(471 \mathrm{MHz}, \mathrm{CDCl}_{3}\right)$

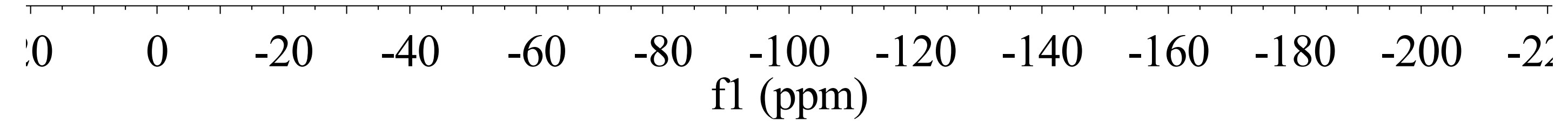




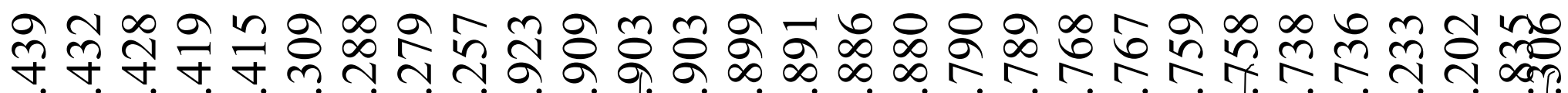

N N N N N N N N N
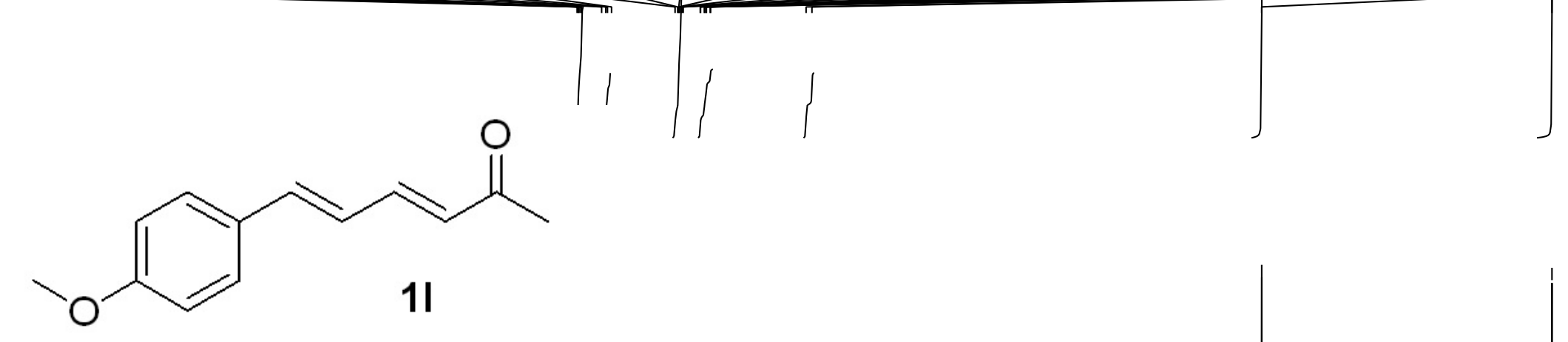

8
$\varnothing$
$\stackrel{0}{1}$
1

${ }^{1} \mathrm{H}$ NMR $\left(500 \mathrm{MHz}, \mathrm{CDCl}_{3}\right)$

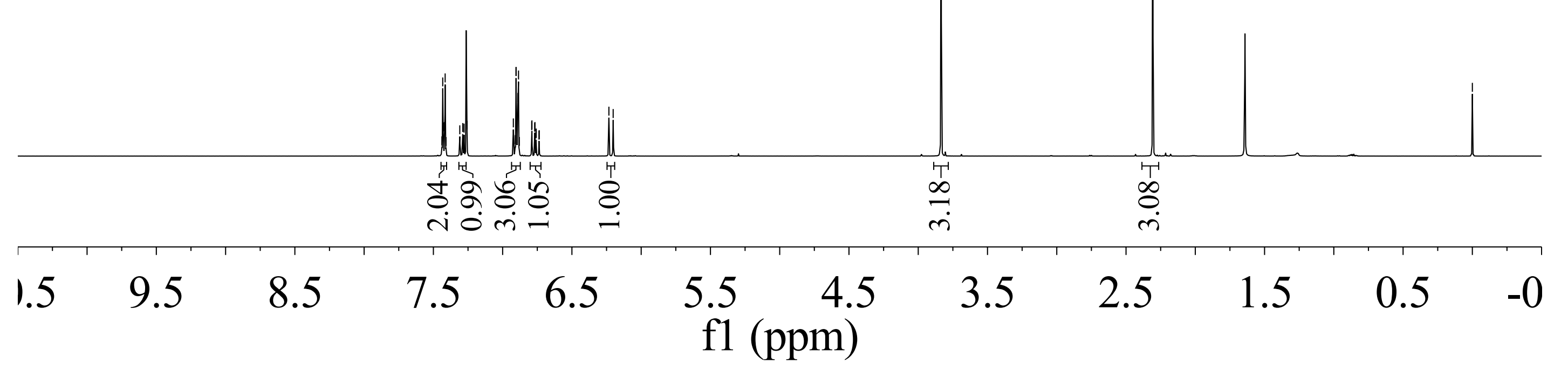



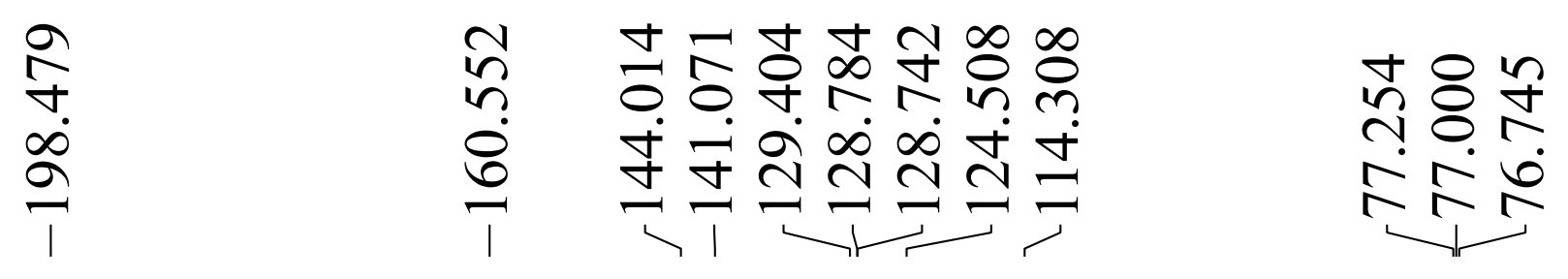

$n$
$n$
$n$
$n$

$\frac{\hat{N}}{\stackrel{1}{1}}$

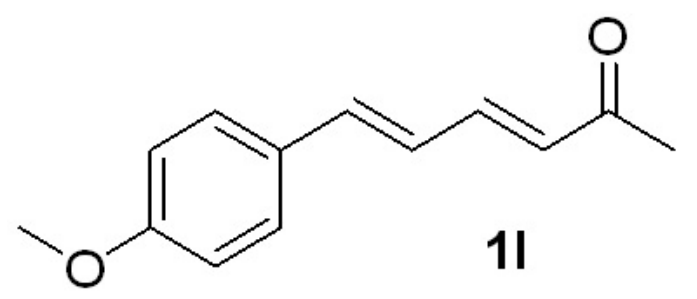

${ }^{13} \mathrm{C}$ NMR $\left(125 \mathrm{MHz}, \mathrm{CDCl}_{3}\right)$

$\begin{array}{lllllllllll}210 & 190 & 170 & 150 & 130 & \begin{array}{c}110 \underset{\mathrm{f} 1(\mathrm{ppm})}{90} \\ \text { (1) }\end{array} & 70 & 50 & 30 & 10 & -10\end{array}$




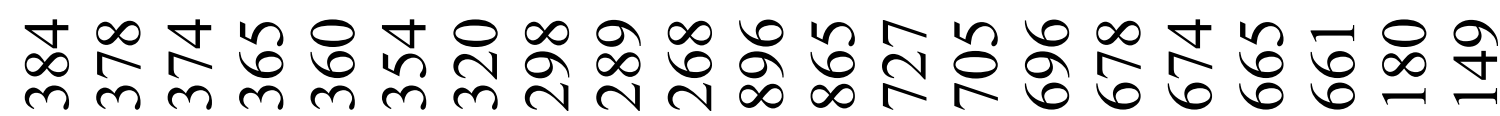

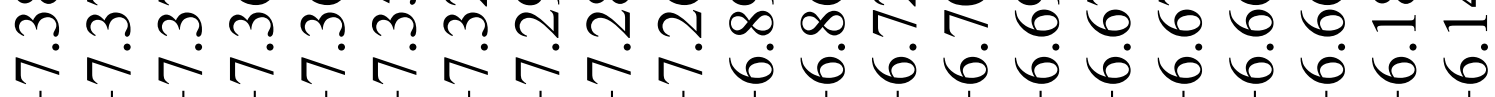

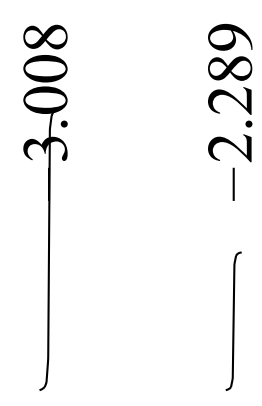

$\underset{\wp}{8}$
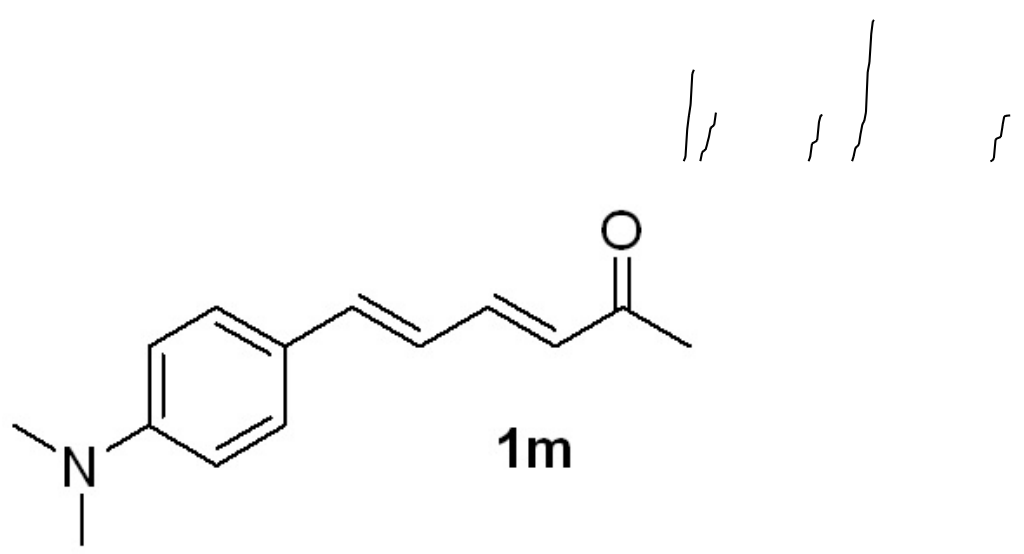

${ }^{1} \mathrm{H}$ NMR $\left(500 \mathrm{MHz}, \mathrm{CDCl}_{3}\right)$

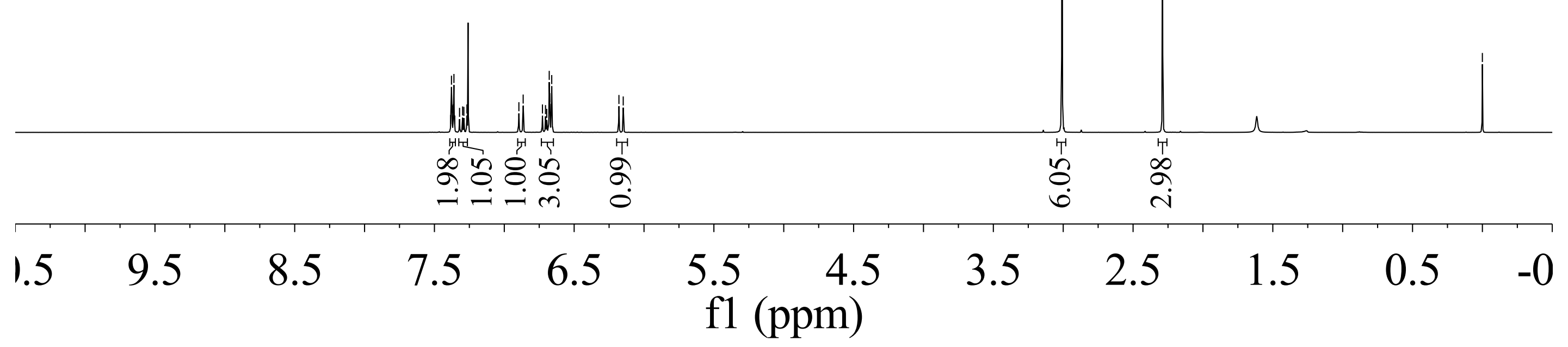



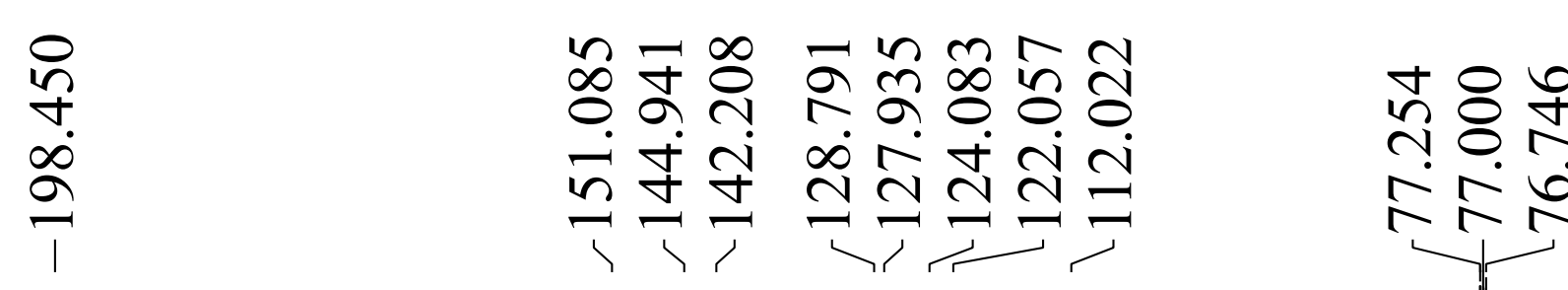

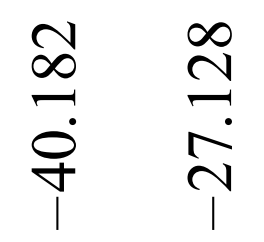

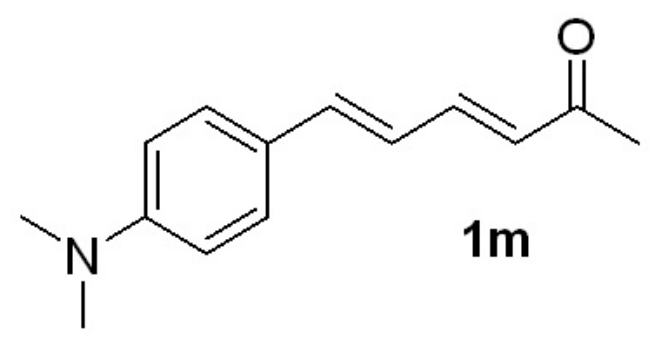

${ }^{13} \mathrm{C}$ NMR $\left(125 \mathrm{MHz}, \mathrm{CDCl}_{3}\right)$

\begin{tabular}{|c|c|c|c|c|c|c|c|c|c|c|}
\hline 210 & 190 & 170 & 150 & 130 & 110 & $\begin{array}{r}90 \\
\mathrm{pm})\end{array}$ & 70 & 50 & 30 & 10 \\
\hline
\end{tabular}




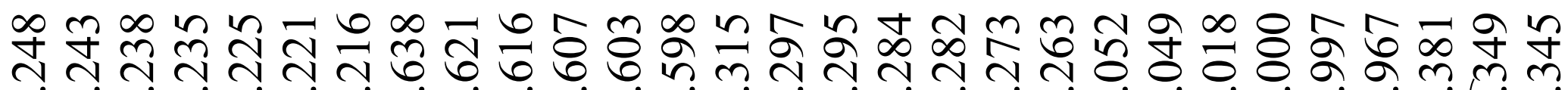

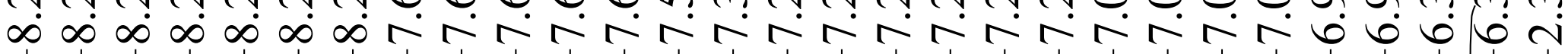

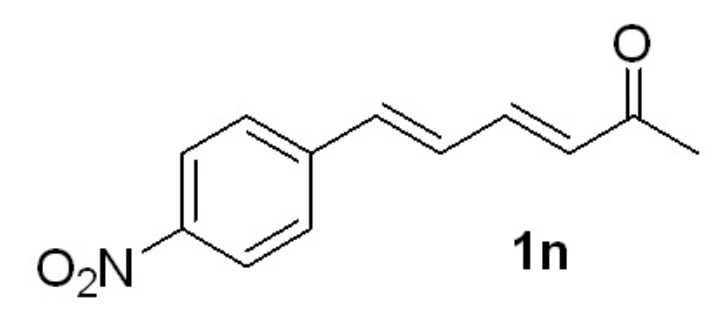

${ }^{1} \mathrm{H}$ NMR $\left(500 \mathrm{MHz}, \mathrm{CDCl}_{3}\right)$

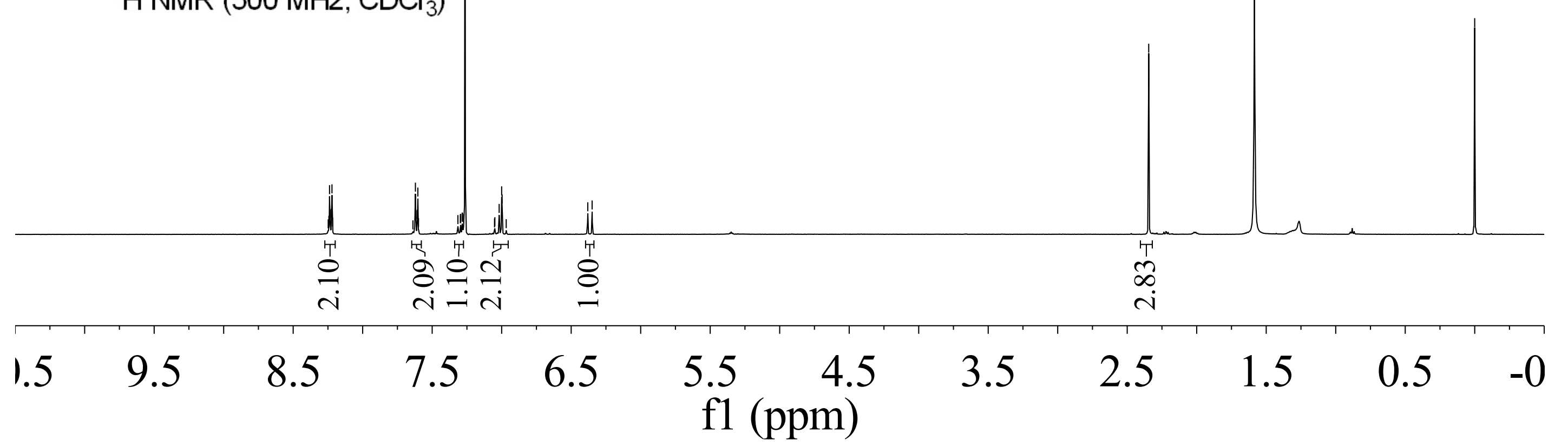




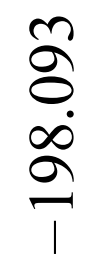

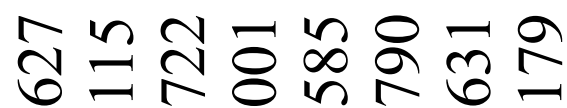

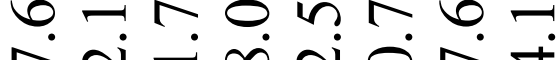

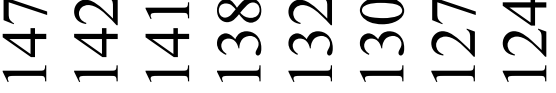

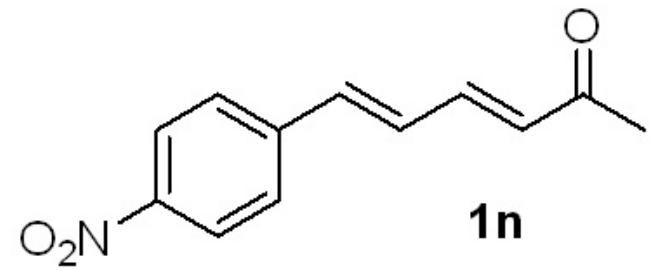

${ }^{13} \mathrm{C}$ NMR $\left(125 \mathrm{MHz}, \mathrm{CDCl}_{3}\right)$

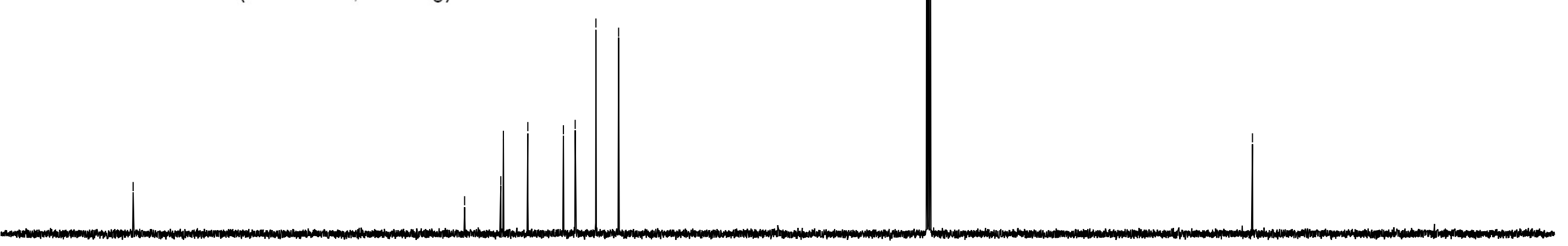

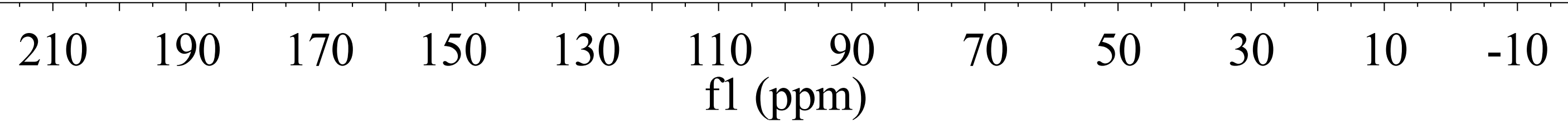


$\frac{8}{8}$
$\frac{1}{1}$

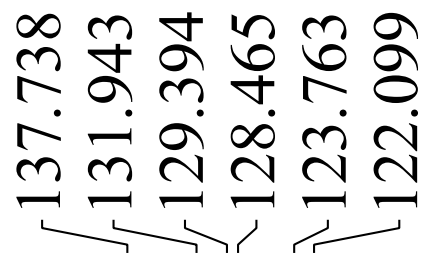

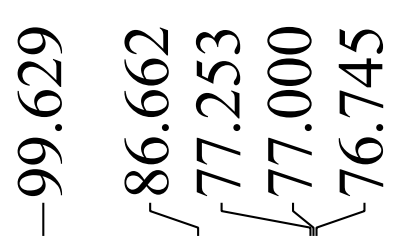

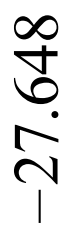

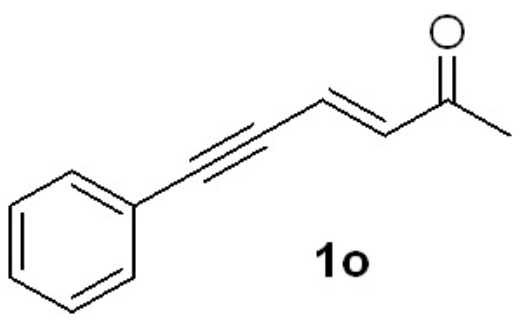

${ }^{13} \mathrm{C} \mathrm{NMR}\left(125 \mathrm{MHz}, \mathrm{CDCl}_{3}\right)$

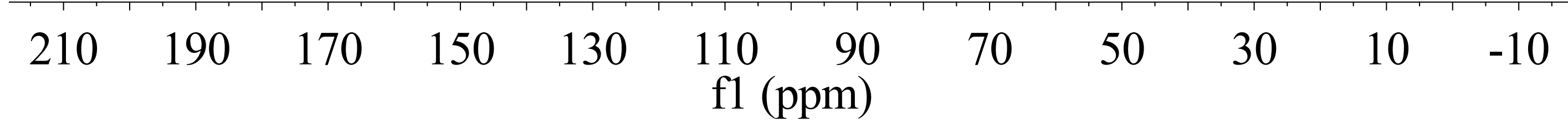




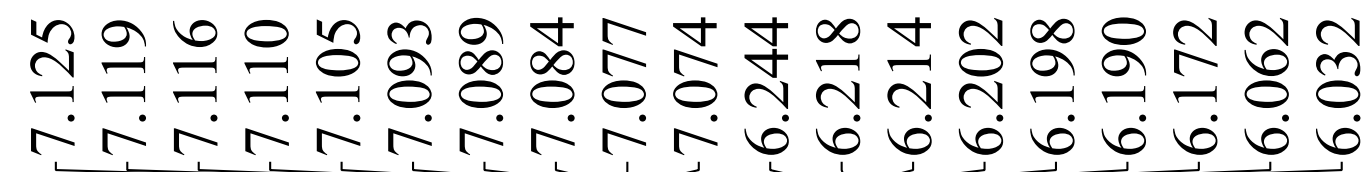

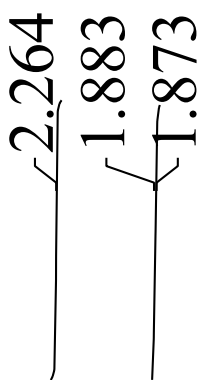

8
0
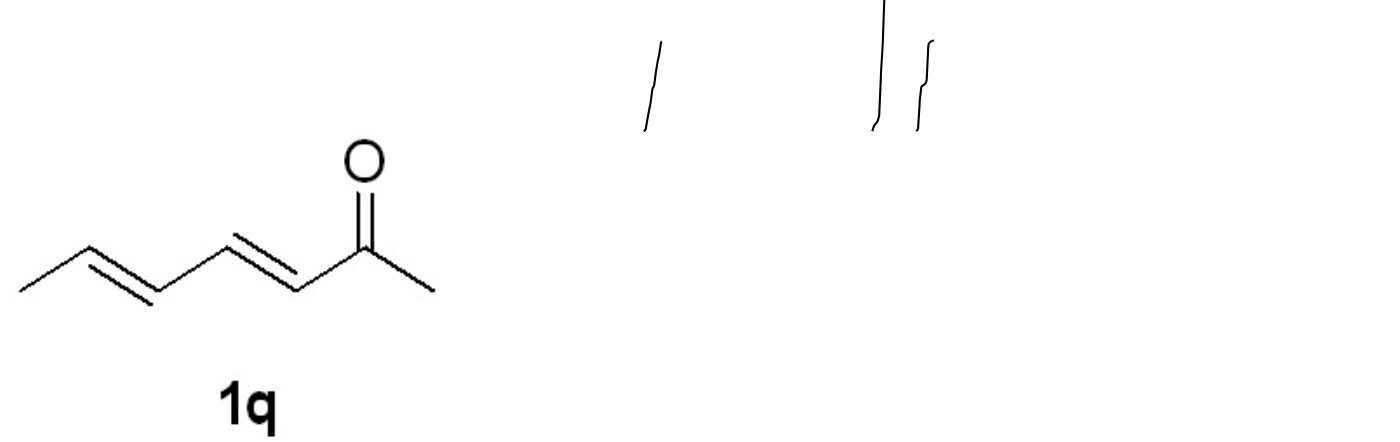

${ }^{1} \mathrm{H}$ NMR $\left(500 \mathrm{MHz}, \mathrm{CDCl}_{3}\right)$

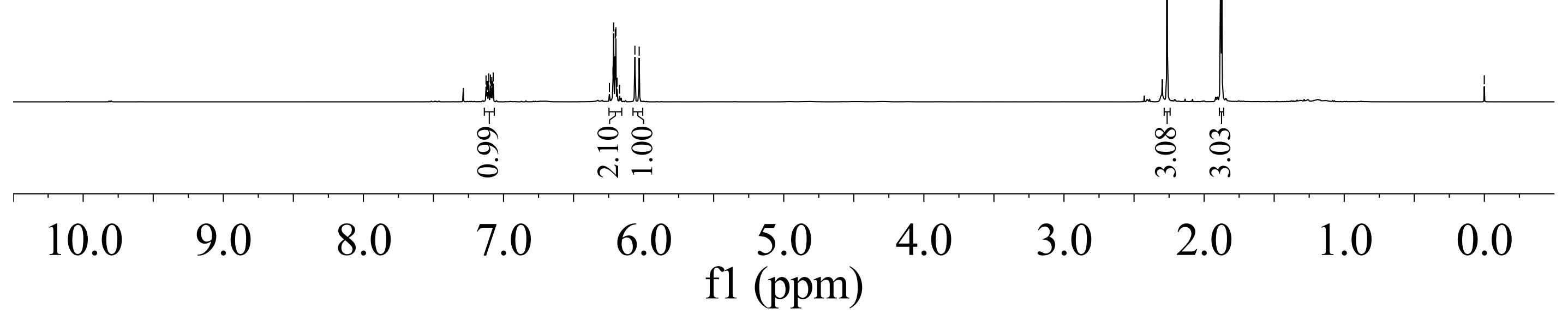



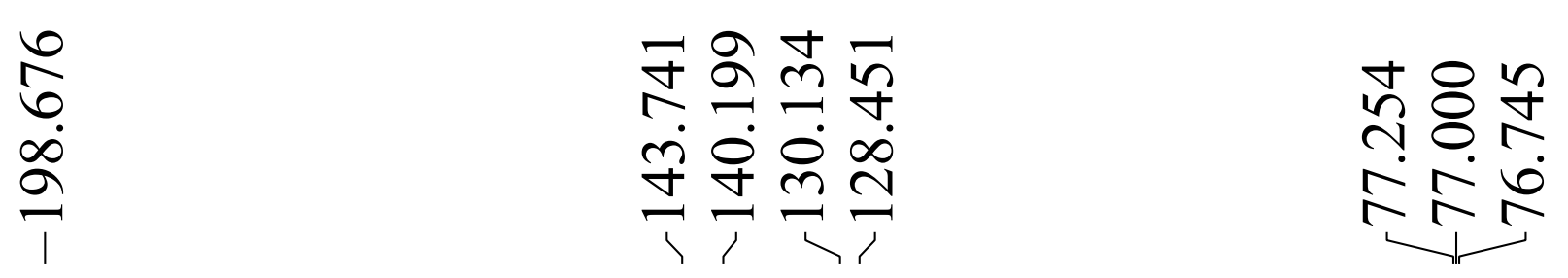

$\begin{array}{ll}n & n \\ 0 & 0 \\ & \infty\end{array}$

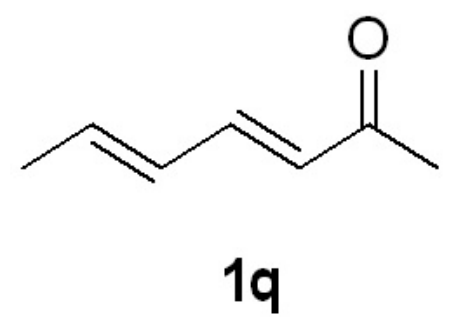

${ }^{13} \mathrm{C}$ NMR $\left(125 \mathrm{MHz}, \mathrm{CDCl}_{3}\right)$

$\begin{array}{lllllll}210 & 190 & 170 & 150 & 130 & 110 & 90 \\ \text { f1 (ppm) }\end{array}$




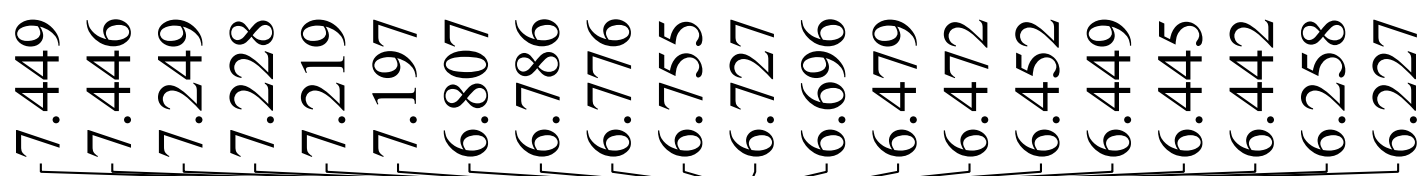

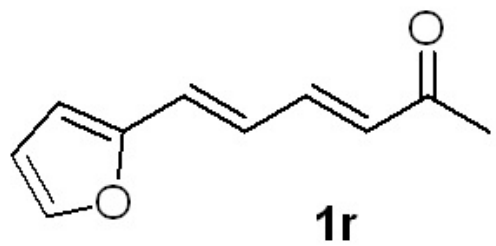

${ }^{1} \mathrm{H}$ NMR $\left(500 \mathrm{MHz}, \mathrm{CDCl}_{3}\right)$

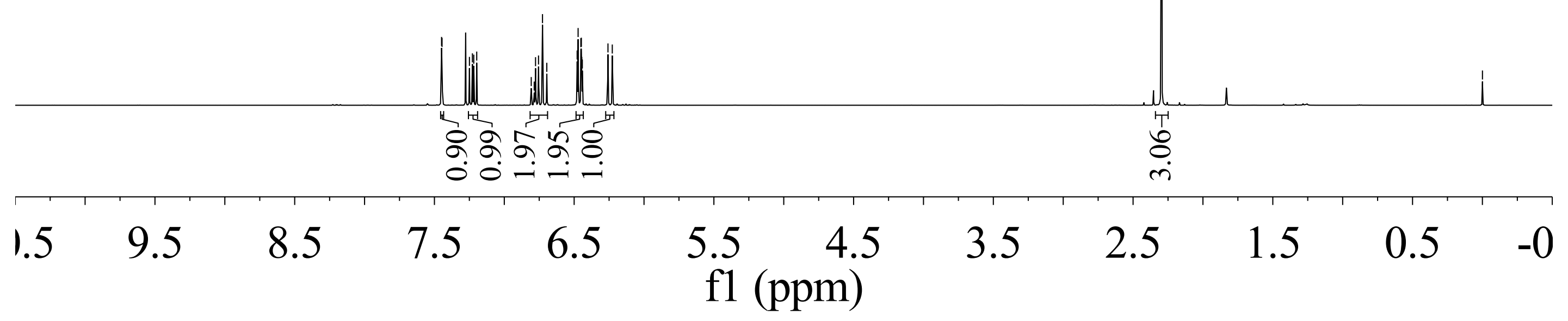




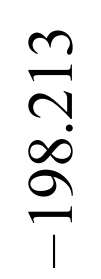
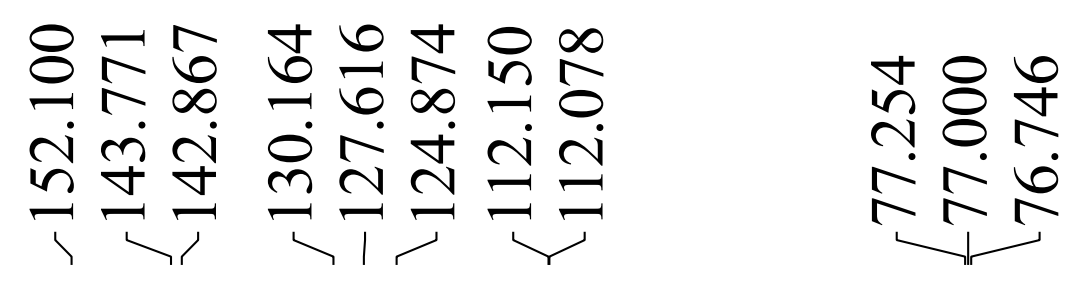

궁

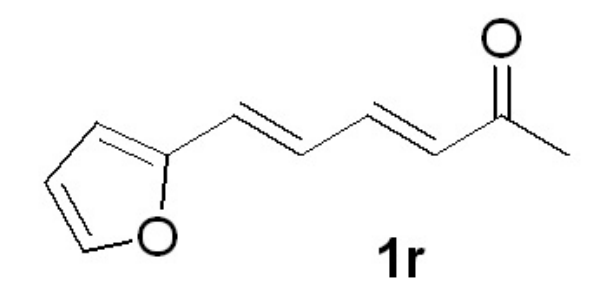

${ }^{13} \mathrm{C}$ NMR $\left(125 \mathrm{MHz}, \mathrm{CDCl}_{3}\right)$

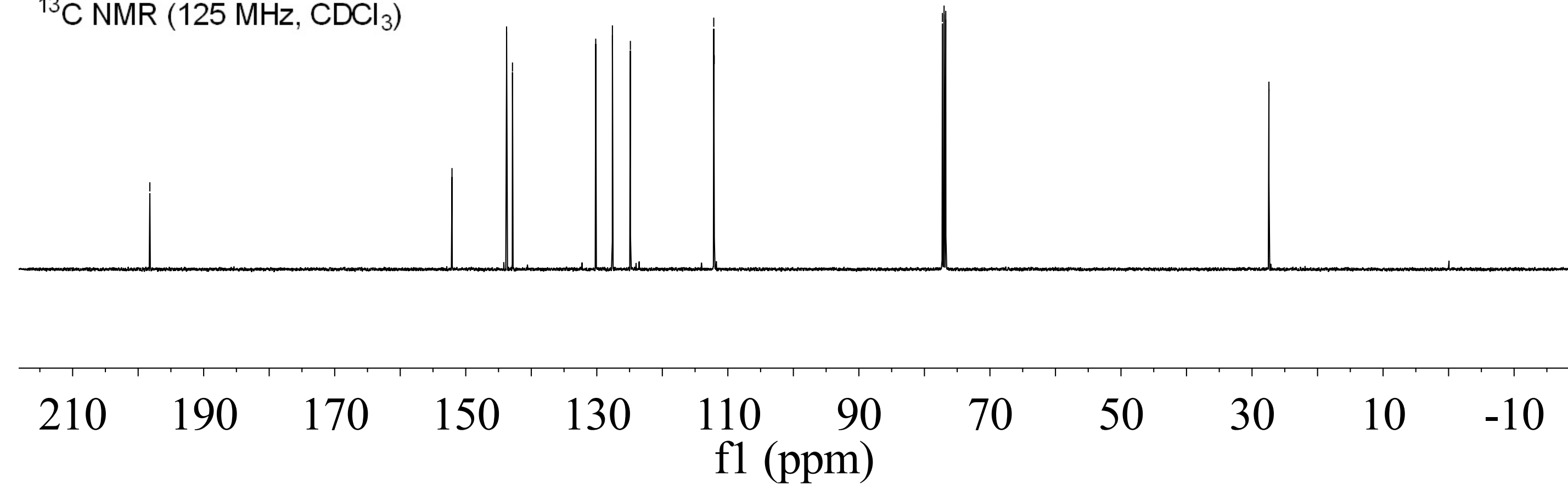




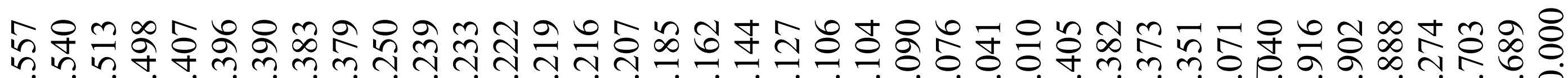

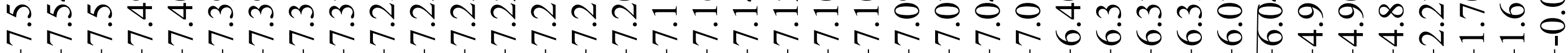
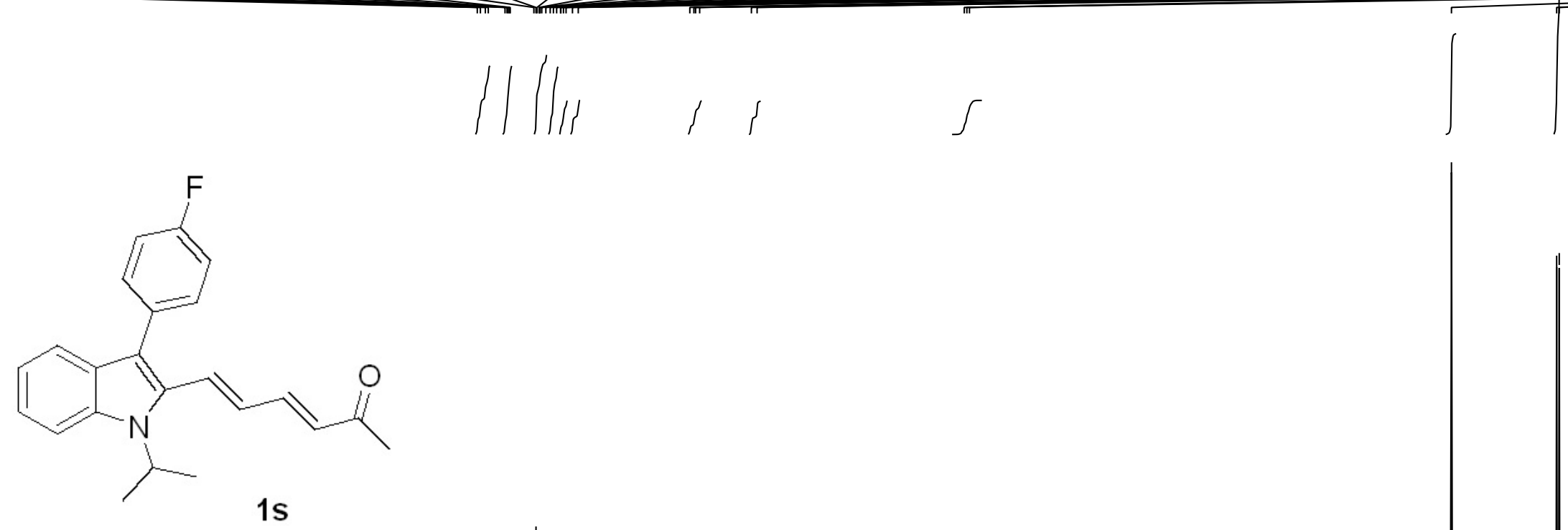

${ }^{1} \mathrm{H} \mathrm{NMR}\left(500 \mathrm{MHz}, \mathrm{CDCl}_{3}\right)$

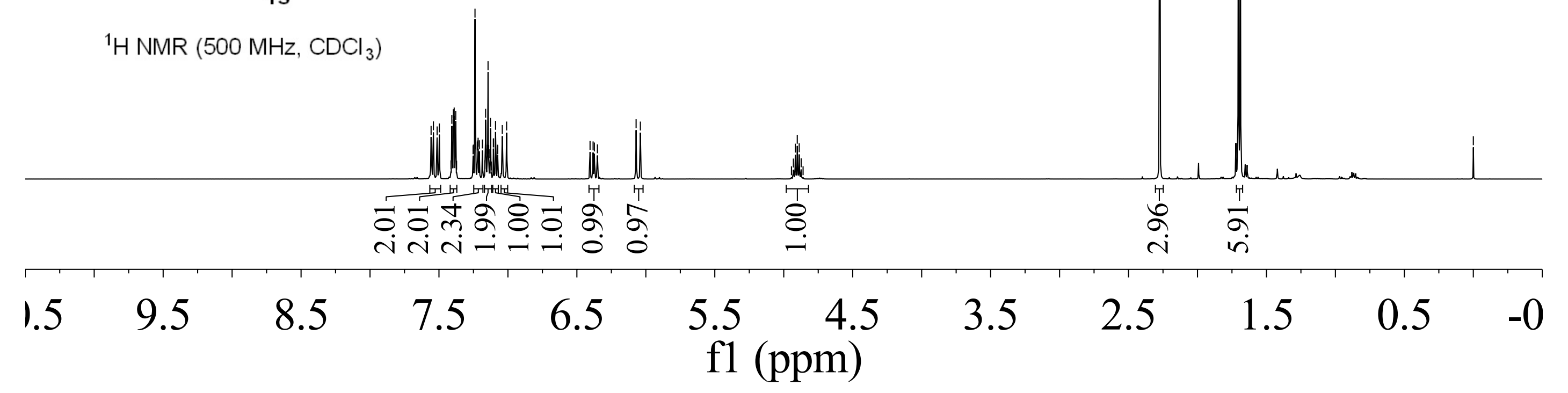

ठ๘ i i i . 


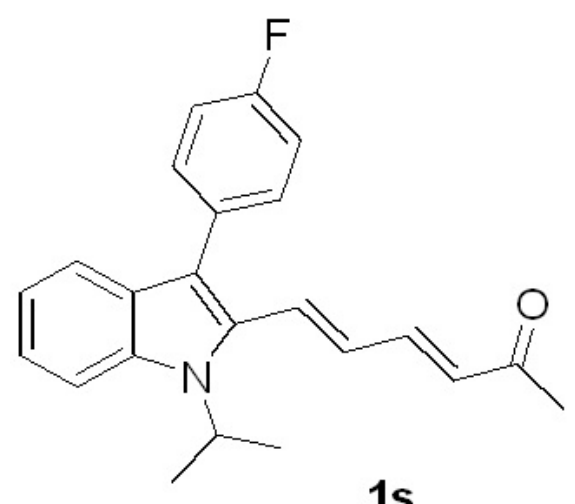

1s

${ }^{13} \mathrm{C}$ NMR $\left(125 \mathrm{MHz}, \mathrm{CDCl}_{3}\right)$

$\begin{array}{rrrrrr}210 & 190 & 170 & 150 & 130 & 110 \quad 90 \\ \text { f1 (ppm) }\end{array}$




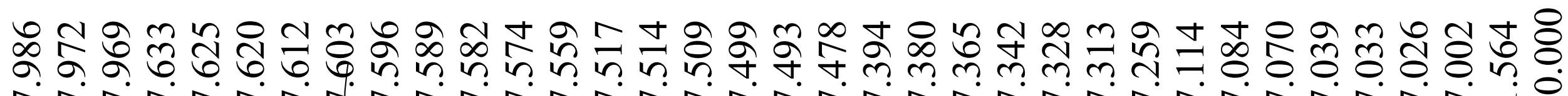

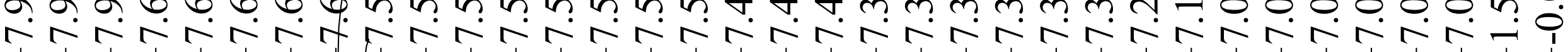
$\left.\int \|\right\}$

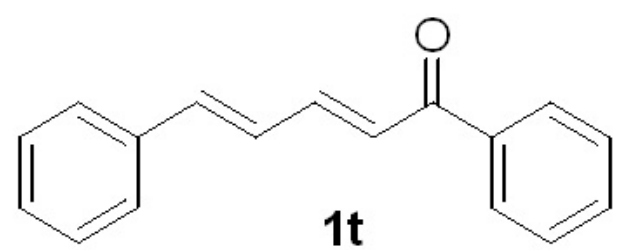

${ }^{1} \mathrm{HNMR}\left(500 \mathrm{MHz}, \mathrm{CDCl}_{3}\right)$

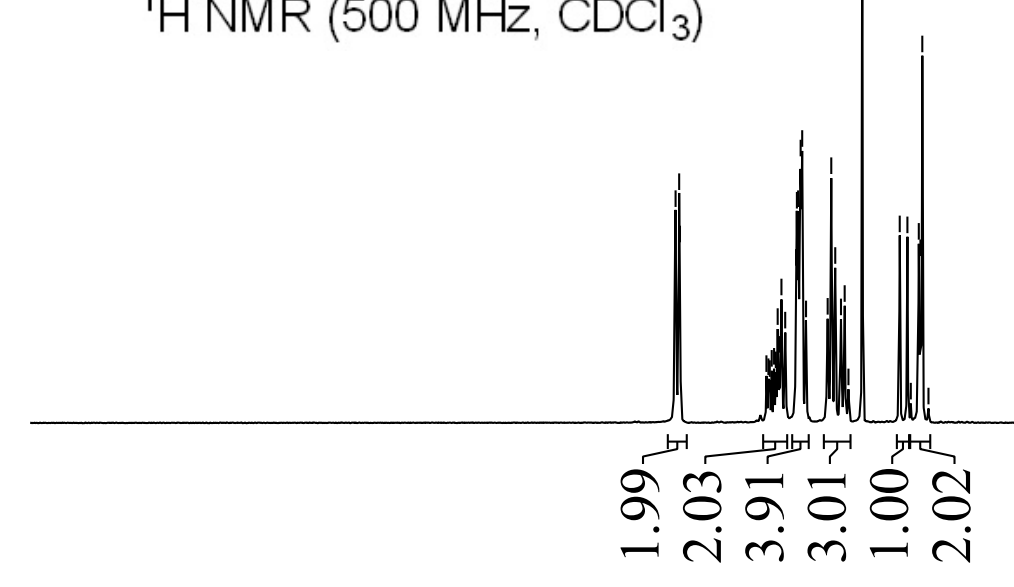

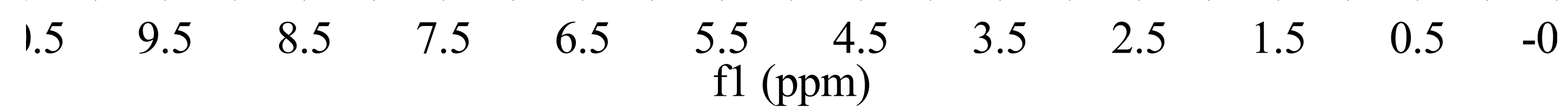



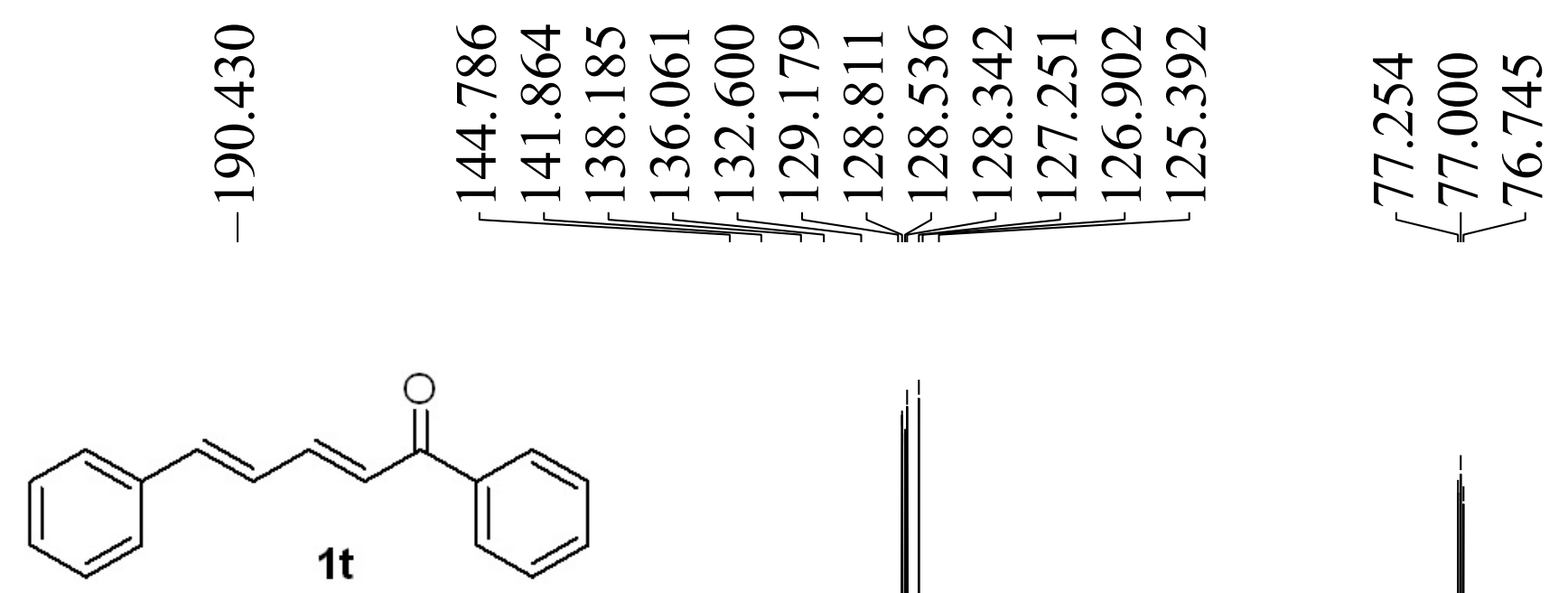

${ }^{13} \mathrm{C}$ NMR $\left(125 \mathrm{MHz}, \mathrm{CDCl}_{3}\right)$

\begin{tabular}{|c|c|c|c|c|c|c|c|c|c|c|}
\hline 210 & 190 & 170 & 150 & 130 & $\begin{array}{l}110 \\
\mathrm{fl}(\mathrm{p}\end{array}$ & $\begin{array}{r}90 \\
\mathrm{pm})\end{array}$ & 70 & 50 & 30 & 10 \\
\hline
\end{tabular}




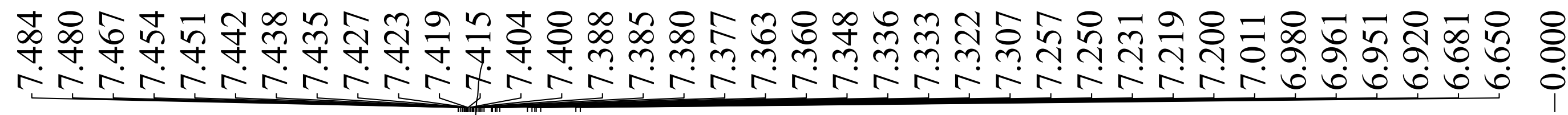

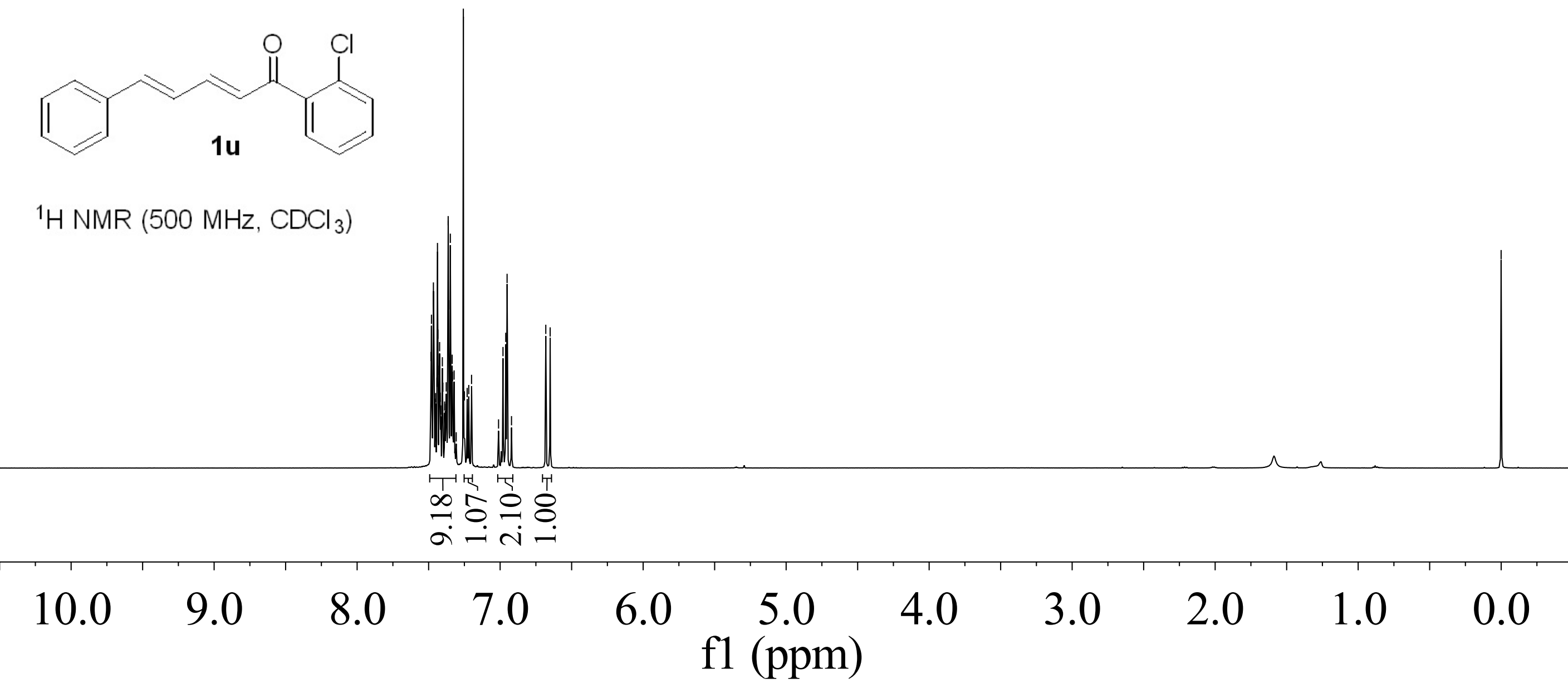



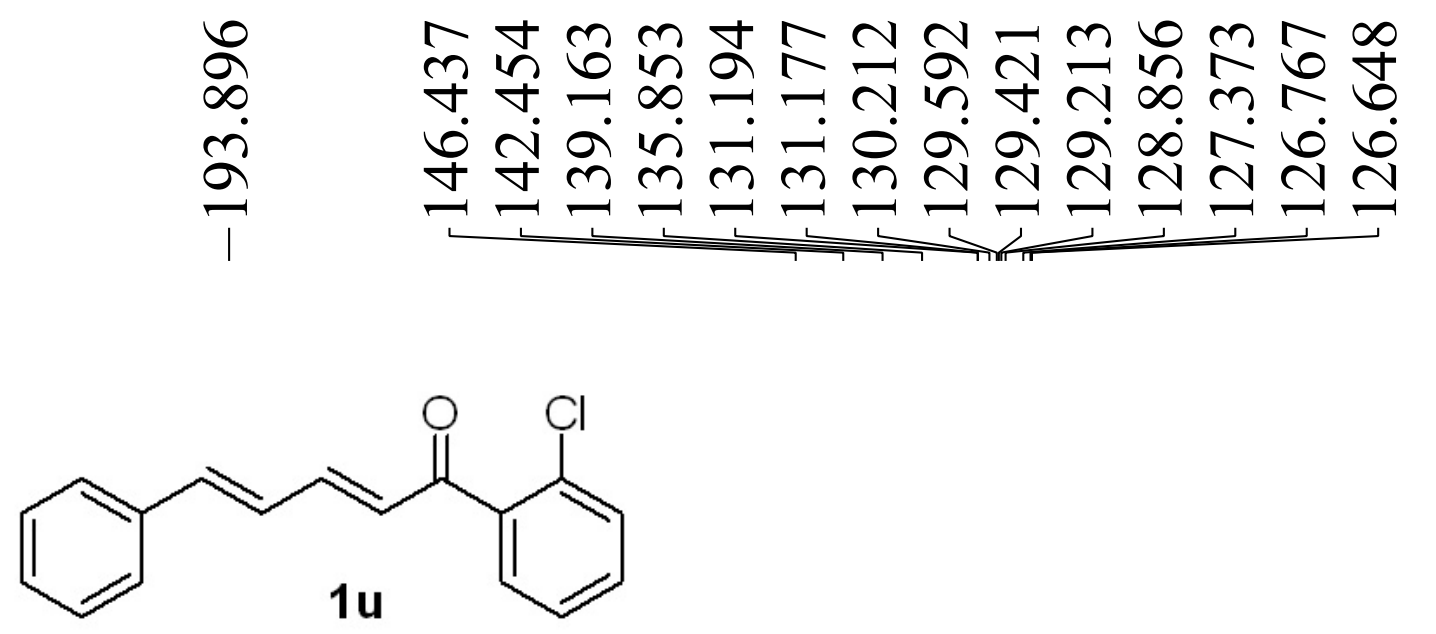

${ }^{13} \mathrm{C} \mathrm{NMR}\left(125 \mathrm{MHz}, \mathrm{CDCl}_{3}\right)$

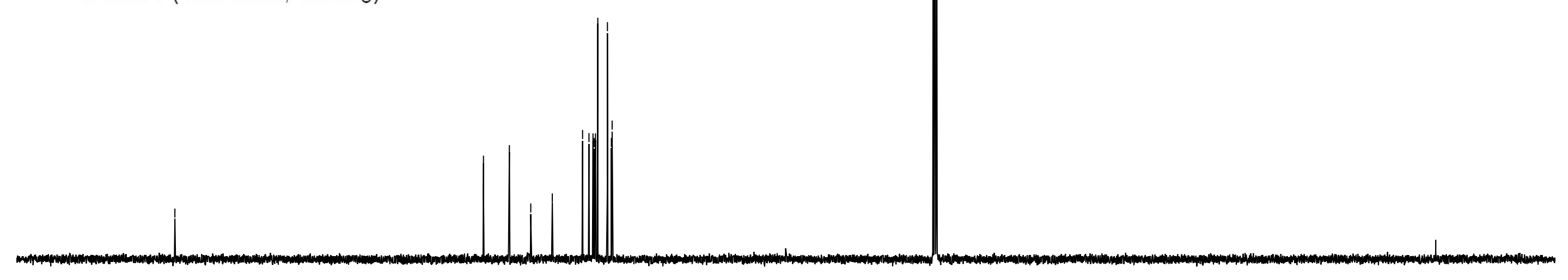

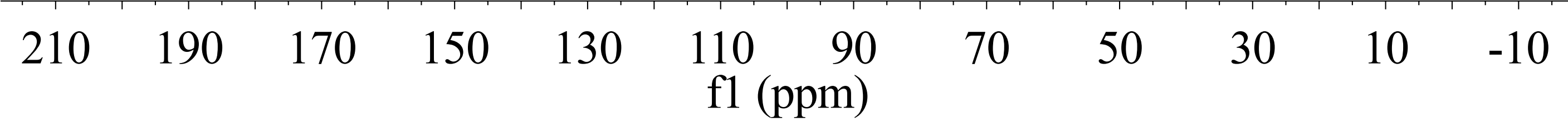




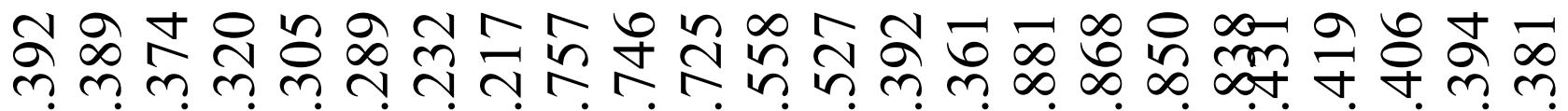
rNN NN

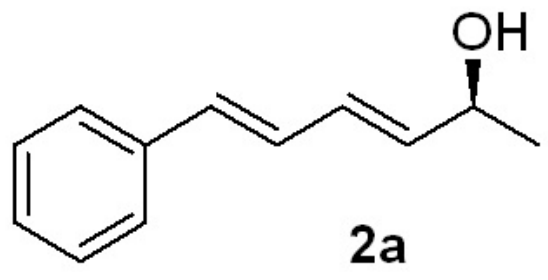

${ }^{1} \mathrm{H}$ NMR $\left(500 \mathrm{MHz}, \mathrm{CDCl}_{3}\right)$
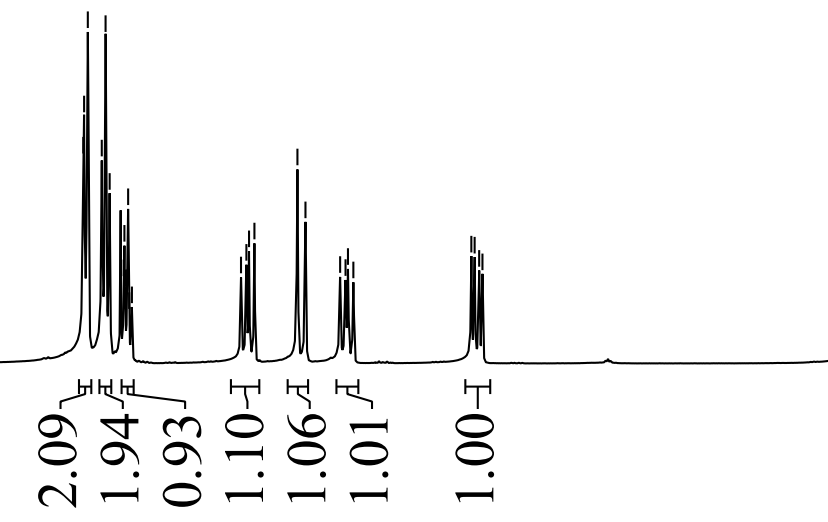

9.5

$8.5 \quad 7.5$

6.5

5.5

fl (ppm) 

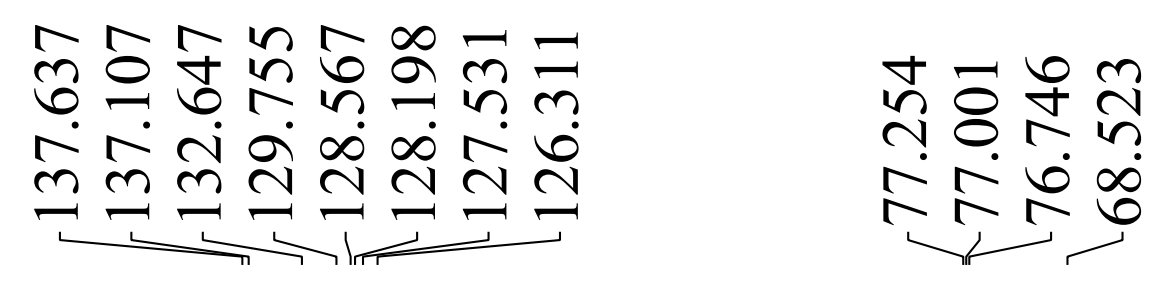

$\stackrel{\bar{o}}{\grave{n}}$

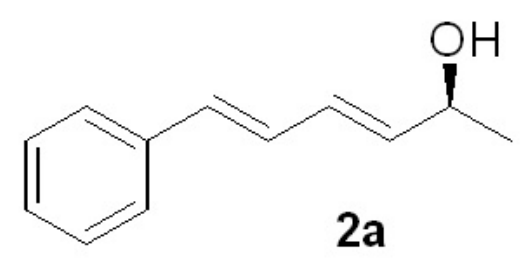

${ }^{13} \mathrm{C} \mathrm{NMR}\left(125 \mathrm{MHz}, \mathrm{CDCl}_{3}\right)$

\begin{tabular}{|c|c|c|c|c|c|c|c|c|c|c|c|}
\hline 210 & 190 & 170 & 150 & 130 & $\begin{array}{l}110 \\
\mathrm{fl}(\mathrm{p}\end{array}$ & $\begin{array}{r}90 \\
\mathrm{pm})\end{array}$ & 70 & 50 & 30 & 10 & -10 \\
\hline
\end{tabular}


广

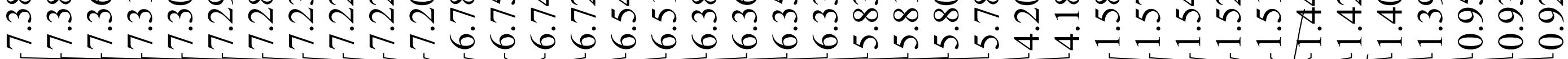
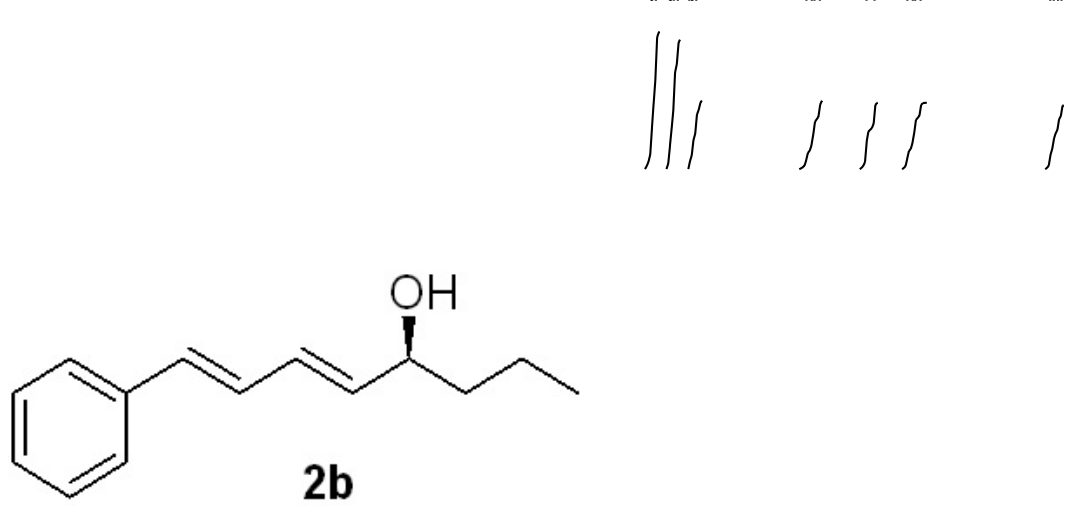

${ }^{1} \mathrm{HNMR}\left(500 \mathrm{MHz}, \mathrm{CDCl}_{3}\right)$

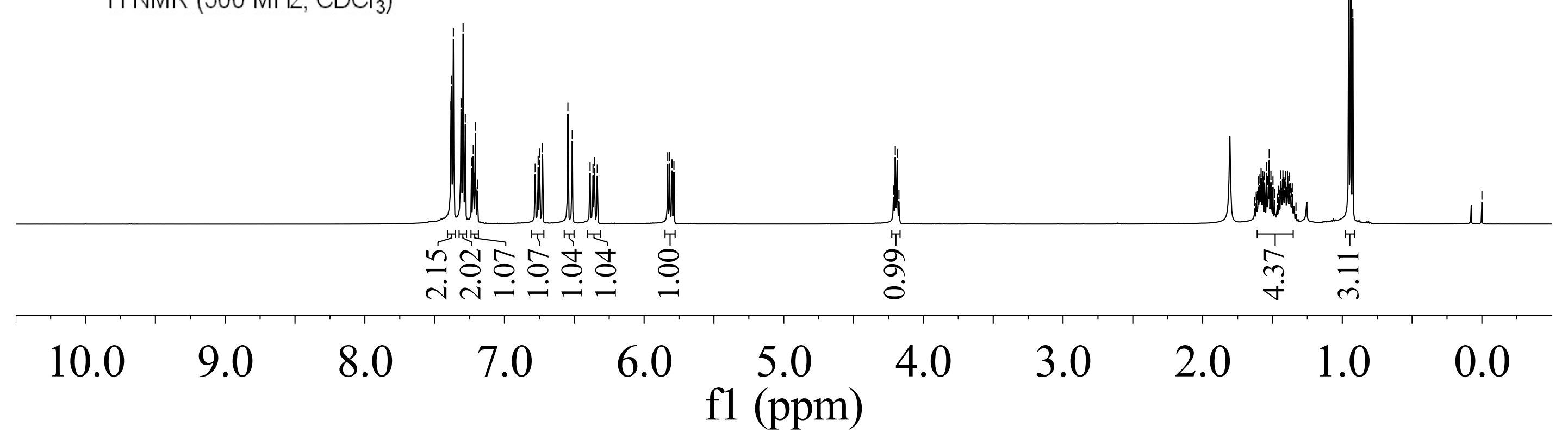



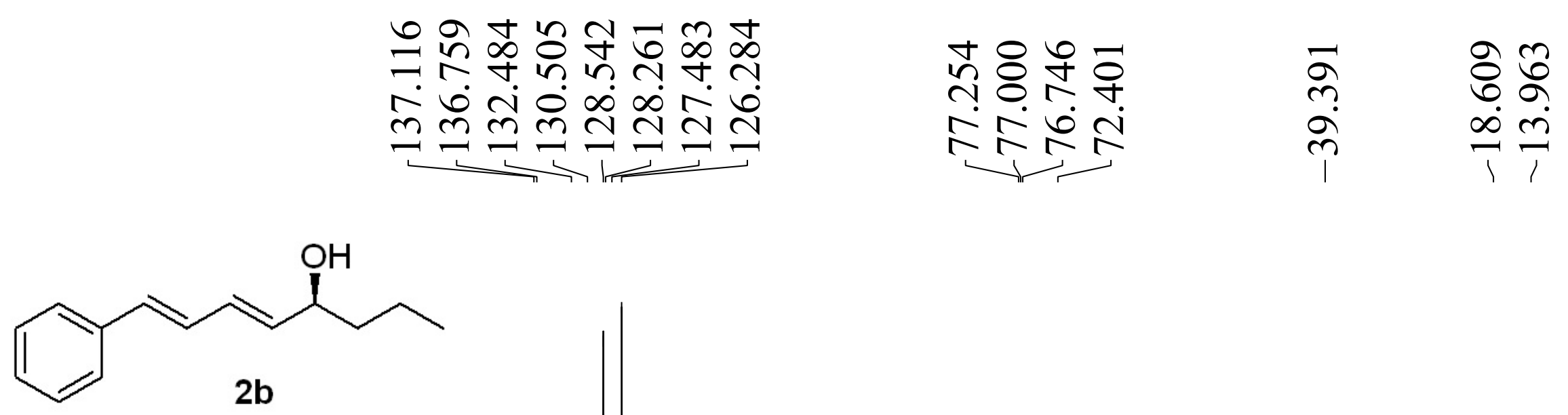

${ }^{13} \mathrm{C}$ NMR $\left(125 \mathrm{MHz}, \mathrm{CDCl}_{3}\right)$

\begin{tabular}{|c|c|c|c|c|c|c|c|c|c|c|}
\hline 210 & 190 & 170 & 150 & 130 & $\begin{array}{c}110 \quad 90 \\
\mathrm{fl}(\mathrm{ppm})\end{array}$ & 70 & 50 & 30 & 10 & -10 \\
\hline
\end{tabular}



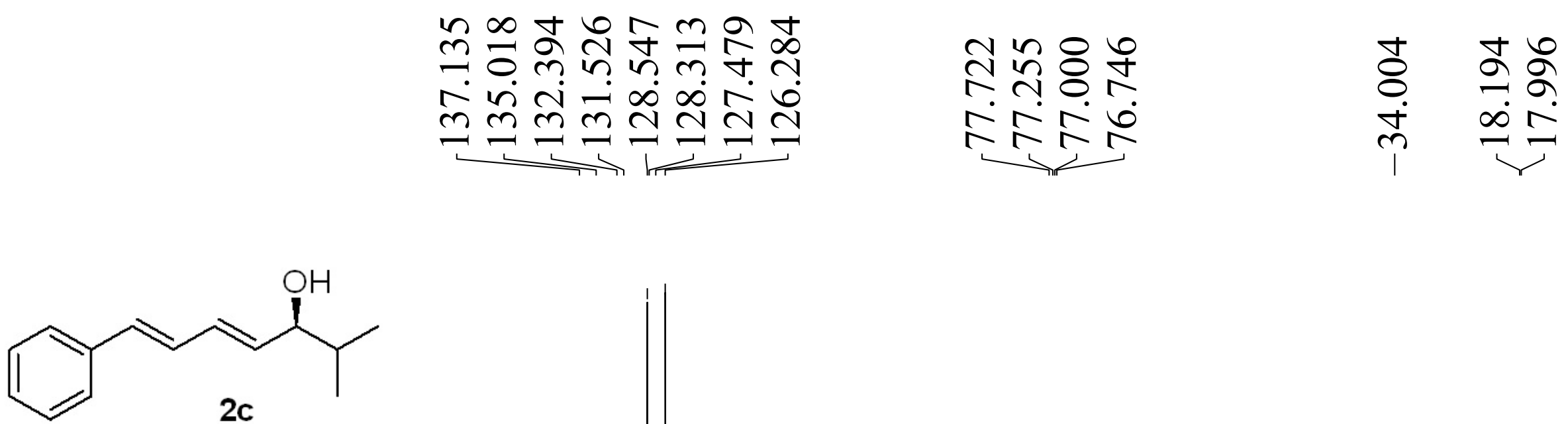

${ }^{13} \mathrm{C}$ NMR $\left(125 \mathrm{MHz}, \mathrm{CDCl}_{3}\right)$

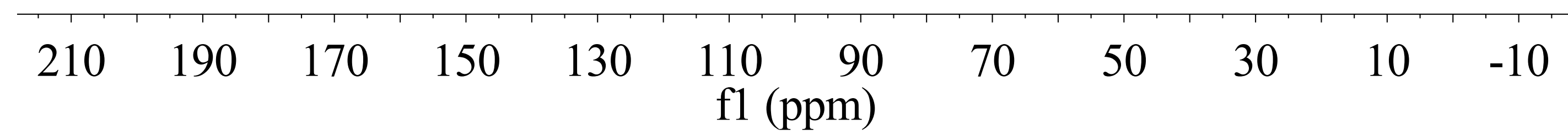




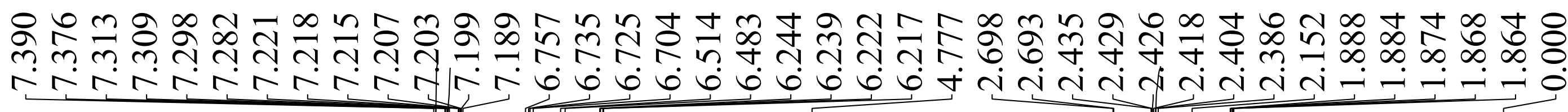

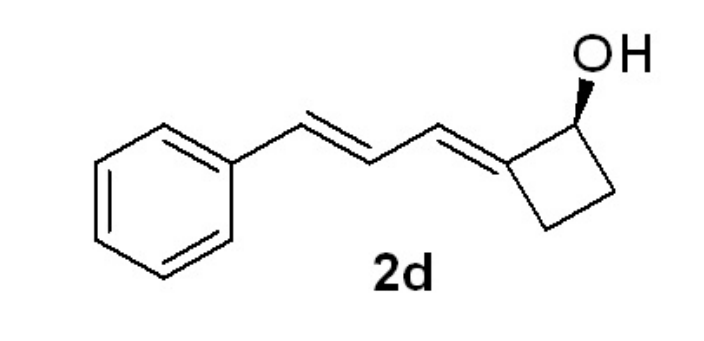

${ }^{1} \mathrm{H}$ NMR $\left(500 \mathrm{MHz}, \mathrm{CDCl}_{3}\right)$
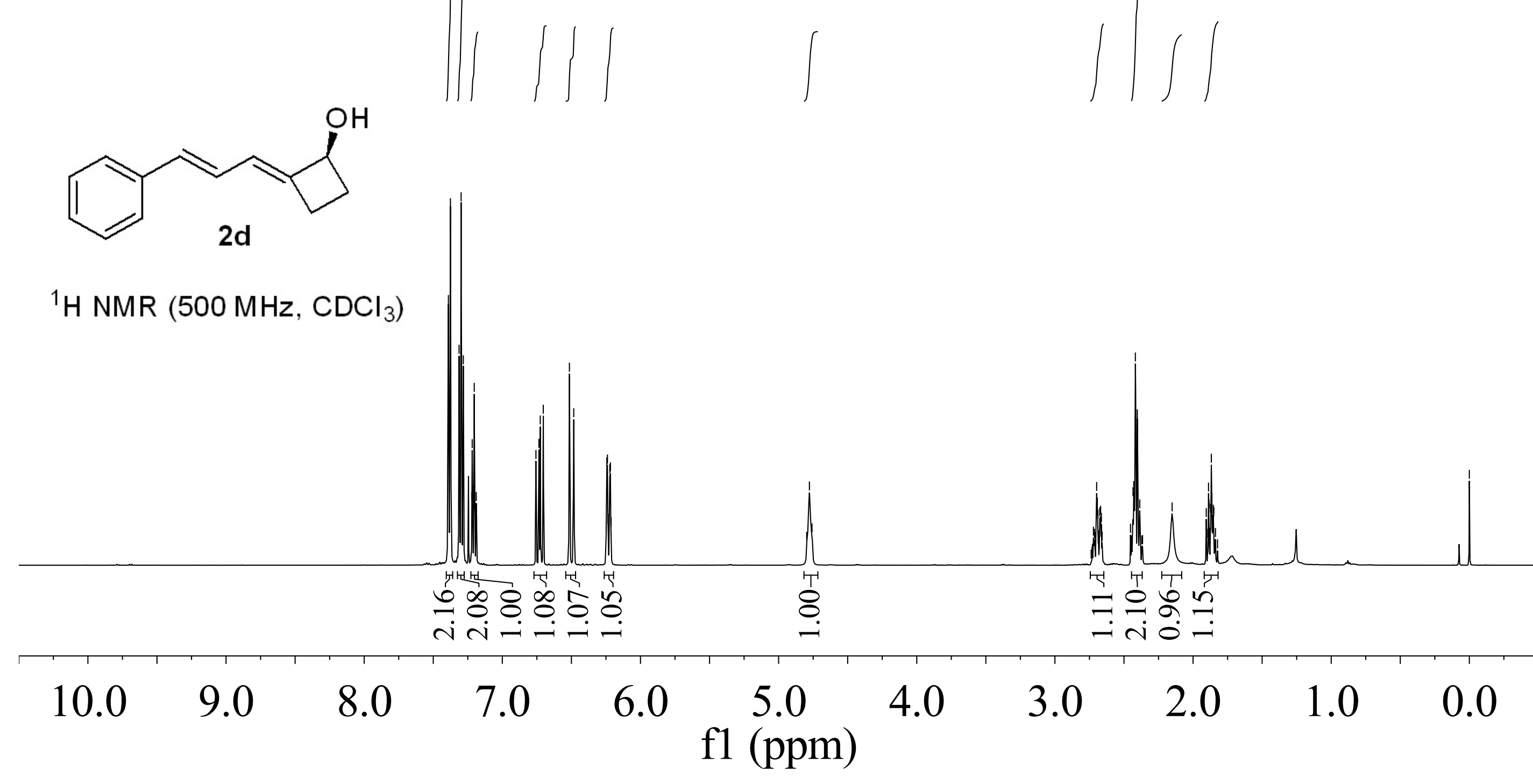

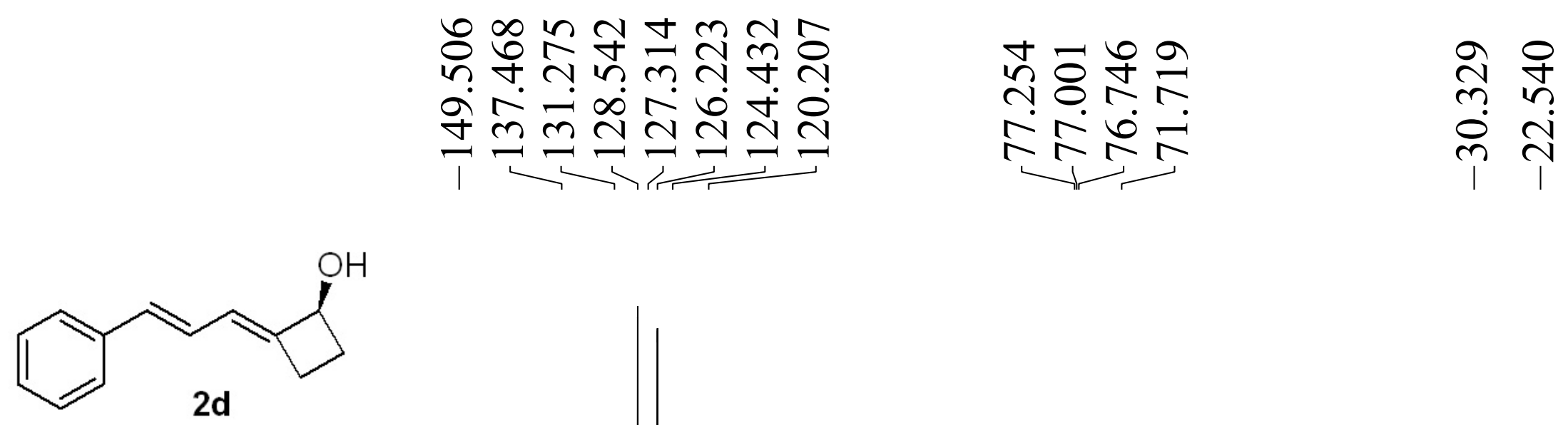

${ }^{13} \mathrm{C} \mathrm{NMR}\left(125 \mathrm{MHz}, \mathrm{CDCl}_{3}\right)$

\begin{tabular}{|c|c|c|c|c|c|c|c|c|c|c|}
\hline 210 & 190 & 170 & 150 & 130 & $\begin{array}{l}110 \\
\mathrm{fl}(\mathrm{p}\end{array}$ & $\begin{array}{r}90 \\
\mathrm{pm})\end{array}$ & 70 & 50 & 30 & 10 \\
\hline
\end{tabular}




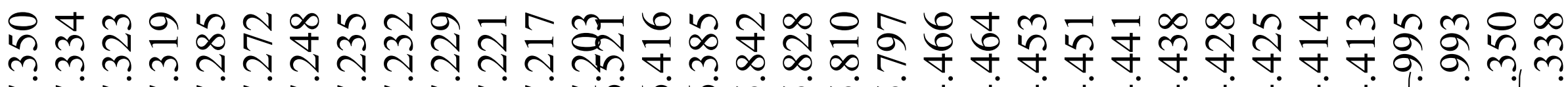

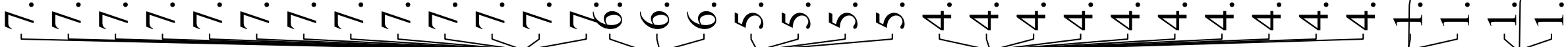
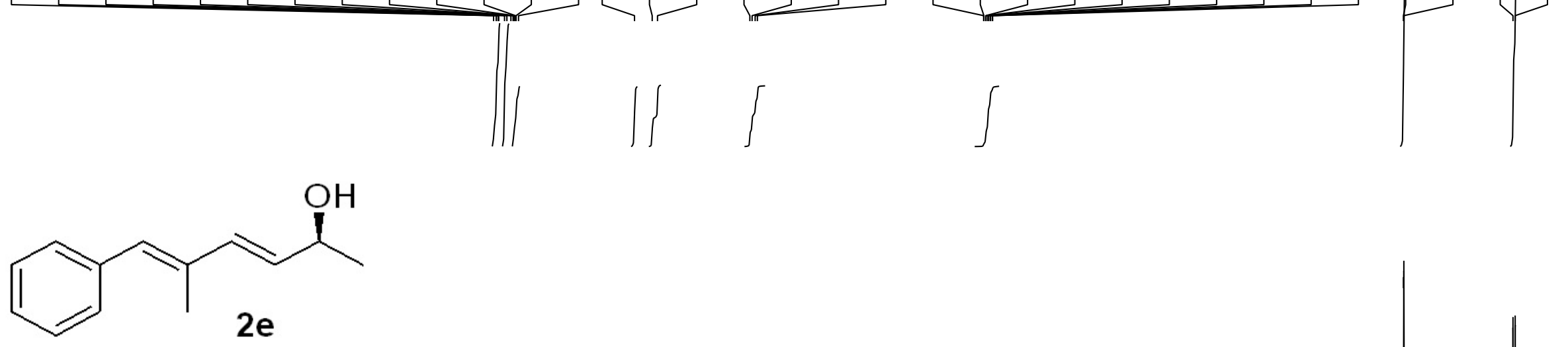

${ }^{1} \mathrm{H}$ NMR $\left(500 \mathrm{MHz}, \mathrm{CDCl}_{3}\right)$

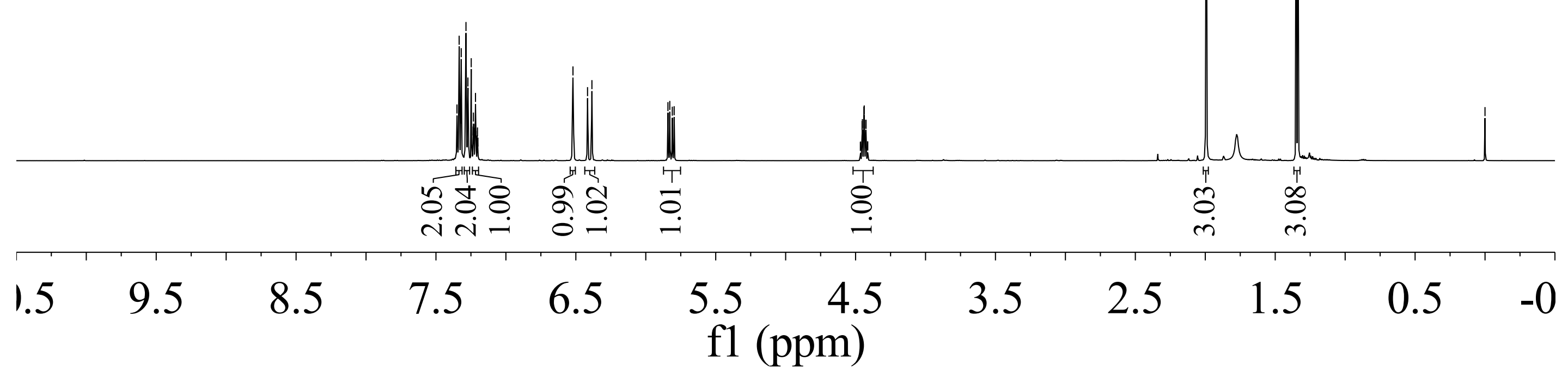



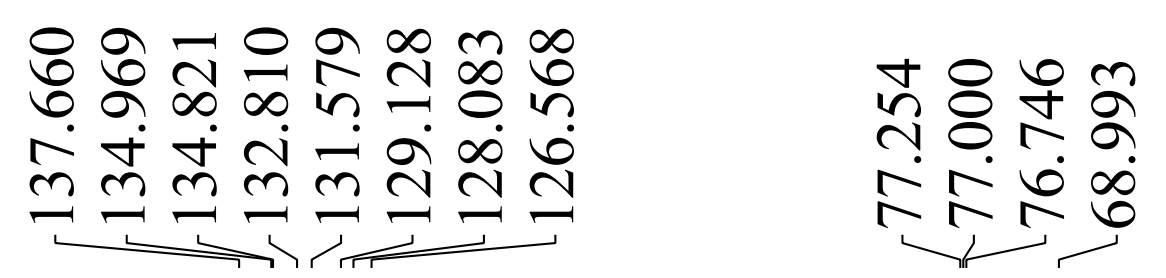

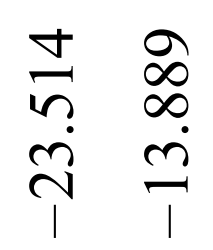

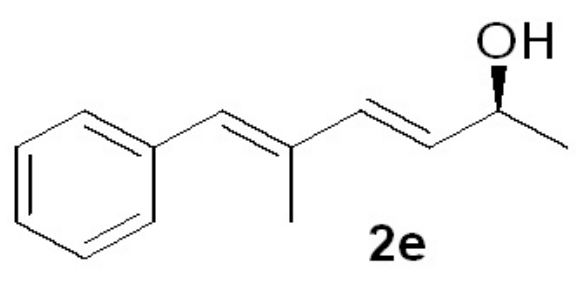

${ }^{13} \mathrm{C}$ NMR $\left(125 \mathrm{MHz}, \mathrm{CDCl}_{3}\right)$
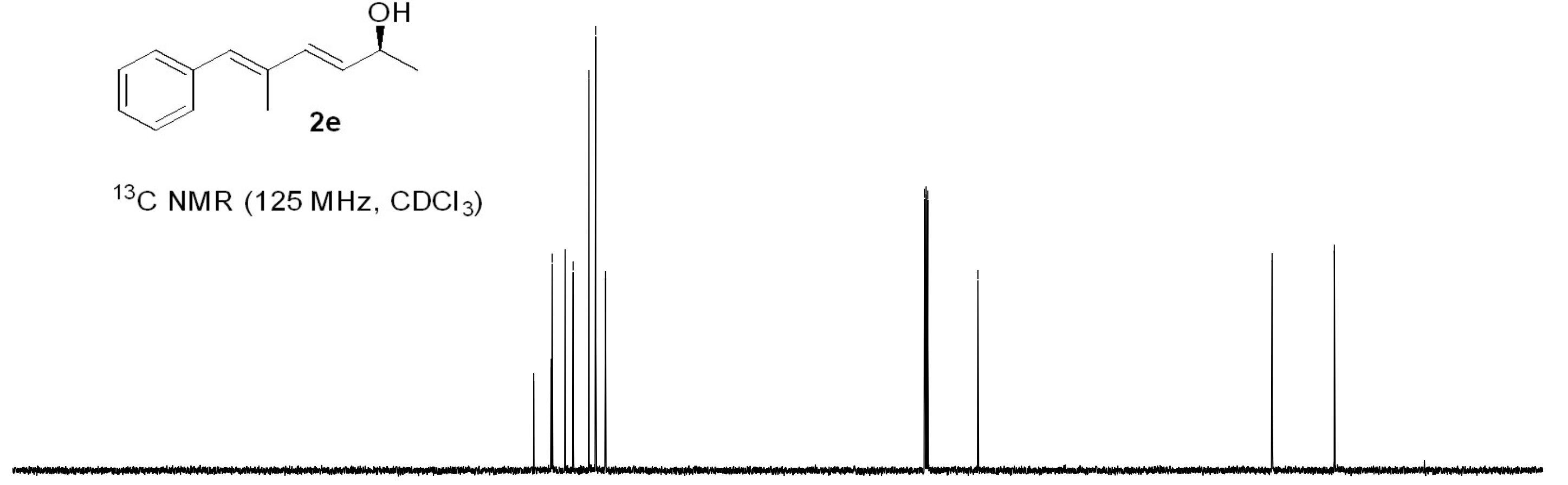

\begin{tabular}{|c|c|c|c|c|c|c|c|c|c|c|}
\hline 210 & 190 & 170 & 150 & 130 & $\begin{array}{c}110 \\
\text { f1 }\end{array}$ & $\begin{array}{c}90 \\
\mathrm{~m})\end{array}$ & 70 & 50 & 30 & 10 \\
\hline
\end{tabular}




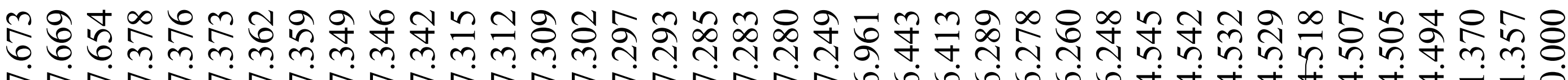

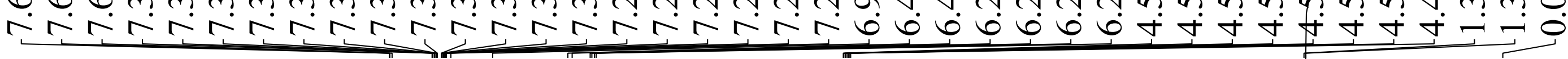
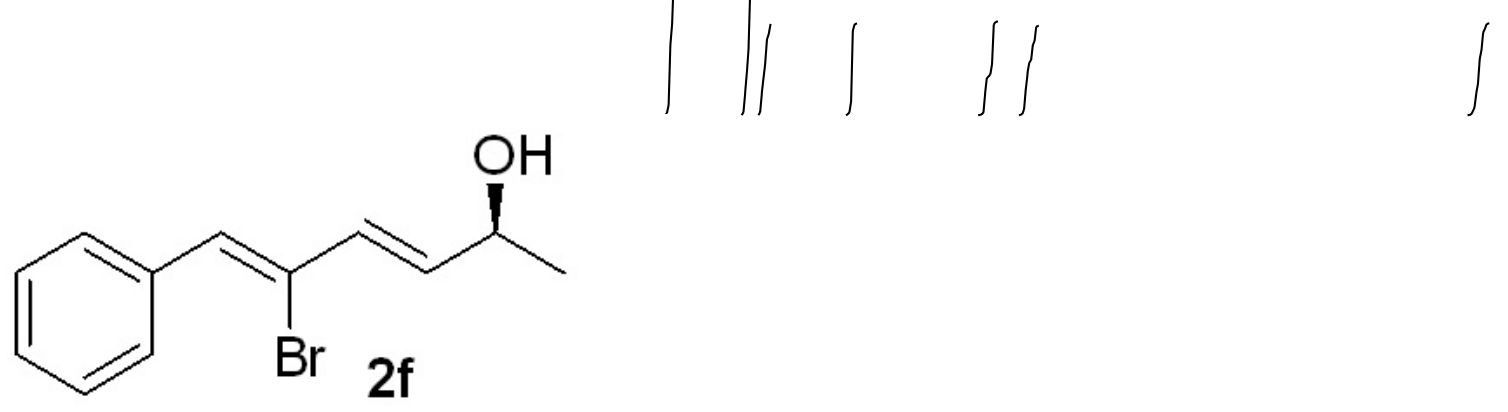

${ }^{1} \mathrm{H}$ NMR (500 MHz, CDCl 3 )

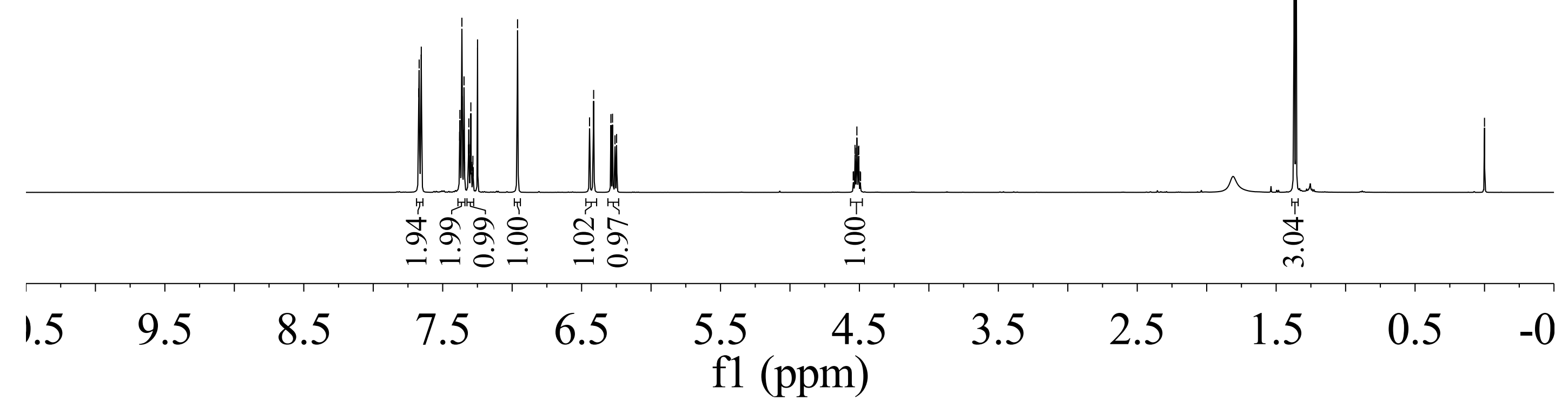



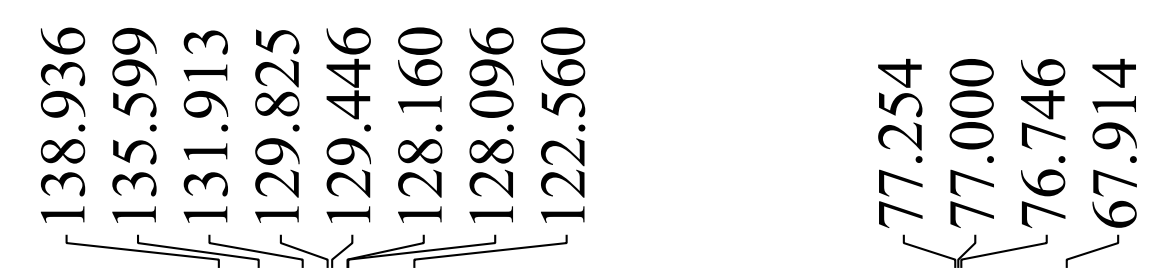

0
ñ
กิ

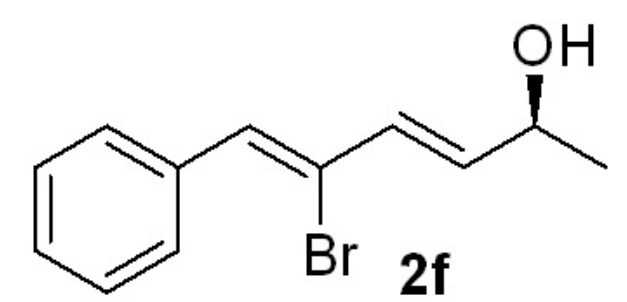

${ }^{13} \mathrm{C} \mathrm{NMR}\left(125 \mathrm{MHz}, \mathrm{CDCl}_{3}\right)$

$\begin{array}{rrrrrr}210 & 190 & 170 & 150 & 130 & 110 \quad 90 \\ \mathrm{fl}(\mathrm{ppm})\end{array}$




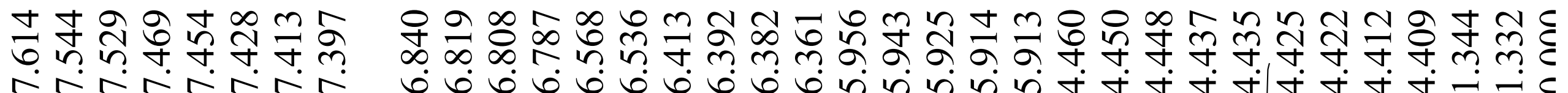
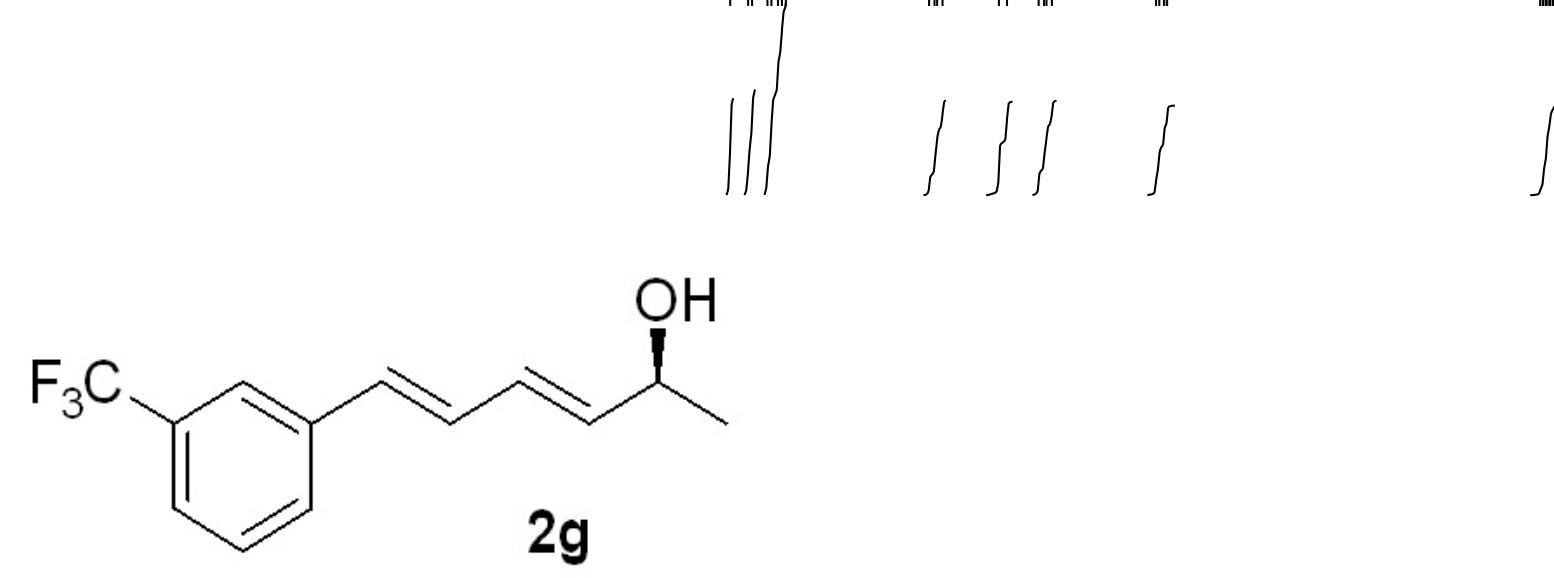

${ }^{1} \mathrm{H}$ NMR $\left(500 \mathrm{MHz}, \mathrm{CDCl}_{3}\right)$

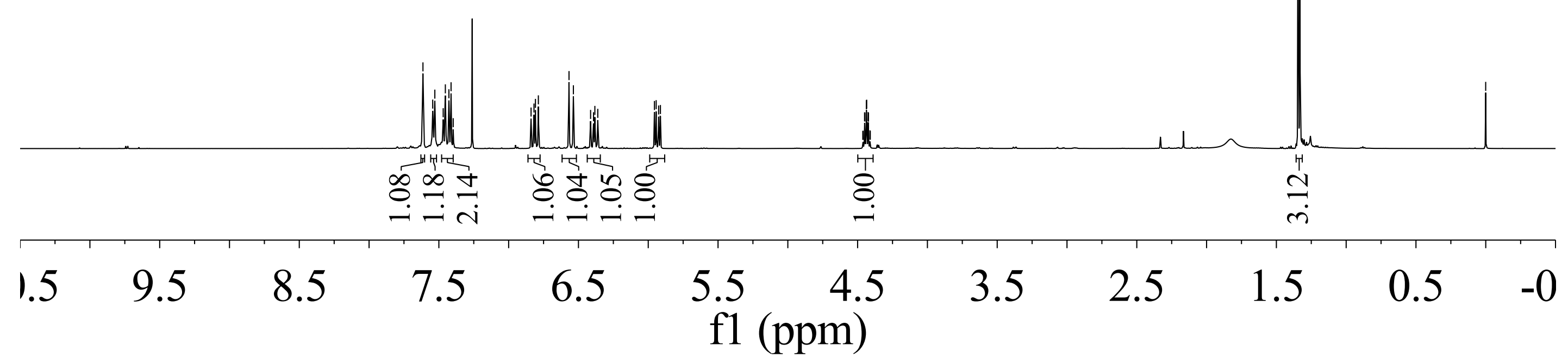




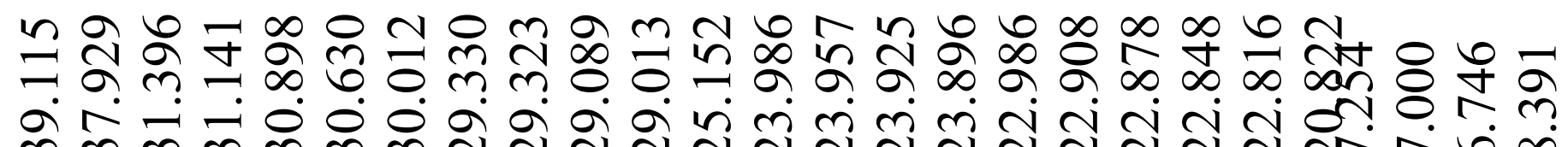
mำ

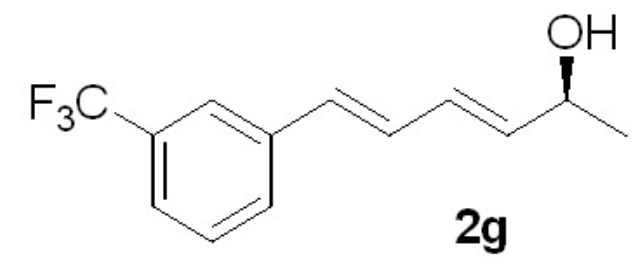

${ }^{13} \mathrm{C} \mathrm{NMR}\left(125 \mathrm{MHz}, \mathrm{CDCl}_{3}\right)$

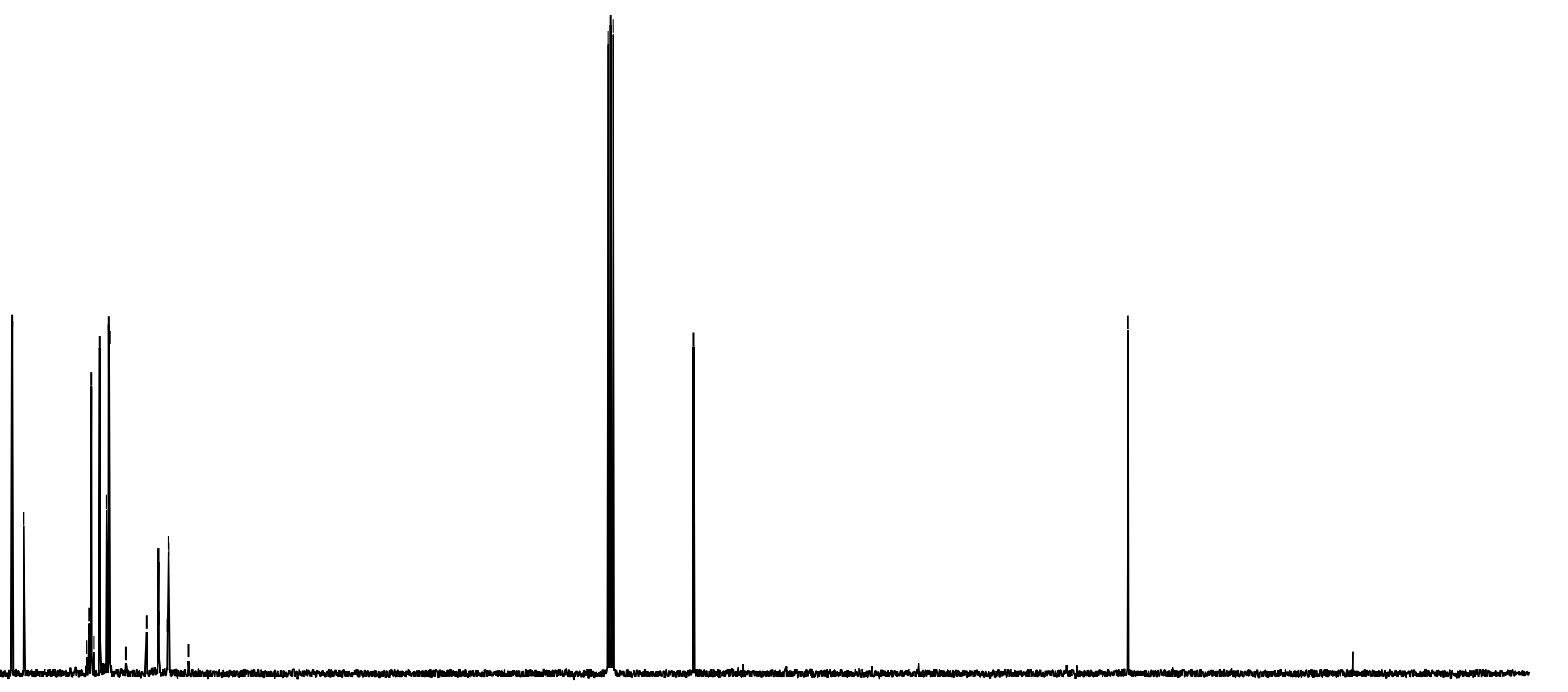

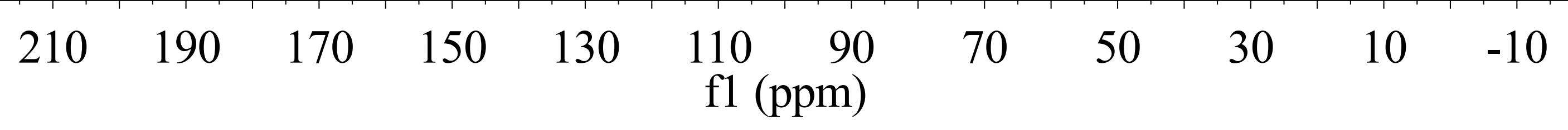


줌ํำ ซ

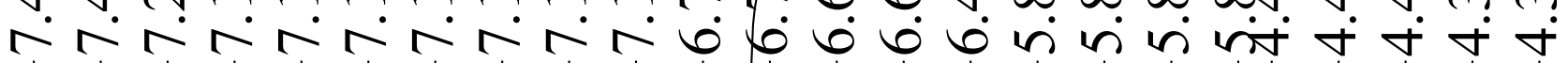
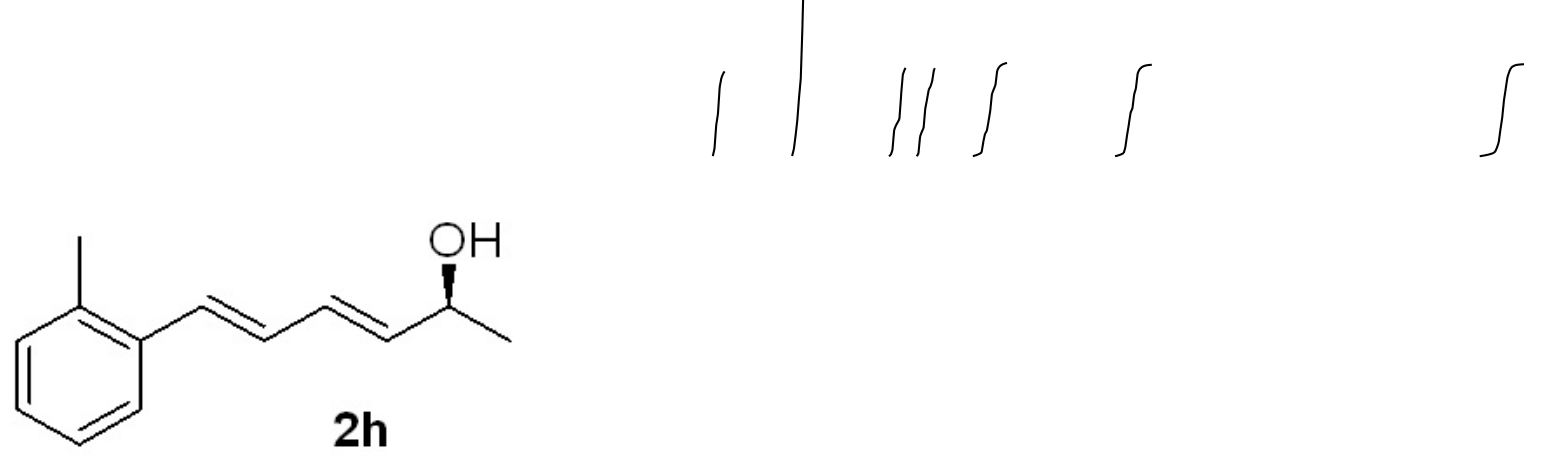

${ }^{1} \mathrm{HNMR}\left(500 \mathrm{MHz}, \mathrm{CDCl}_{3}\right)$

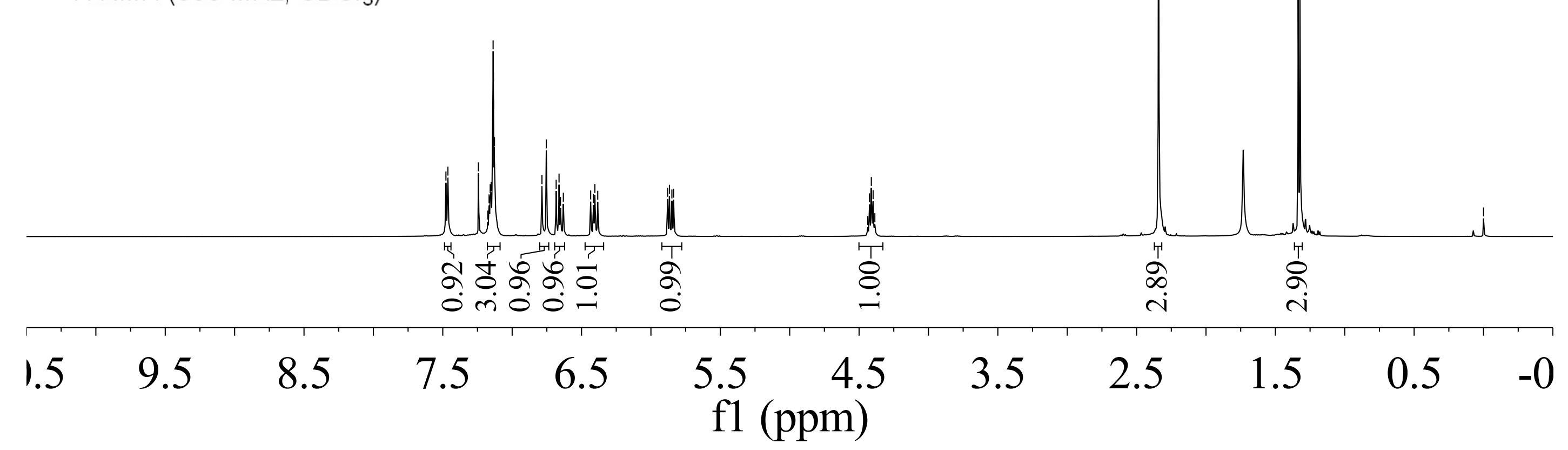



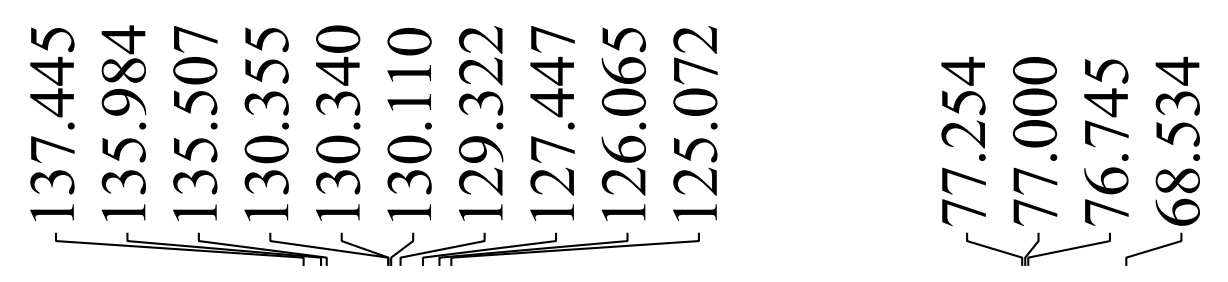

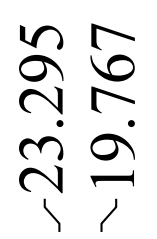

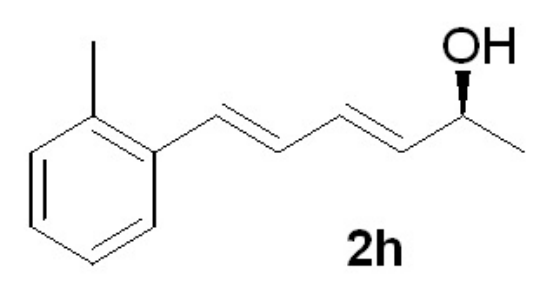

${ }^{13} \mathrm{C}$ NMR $\left(125 \mathrm{MHz}, \mathrm{CDCl}_{3}\right)$

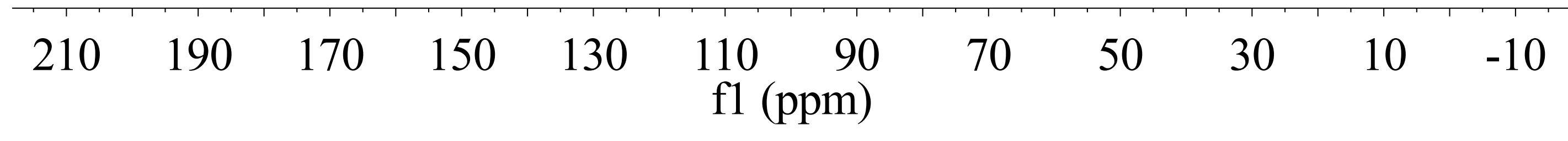



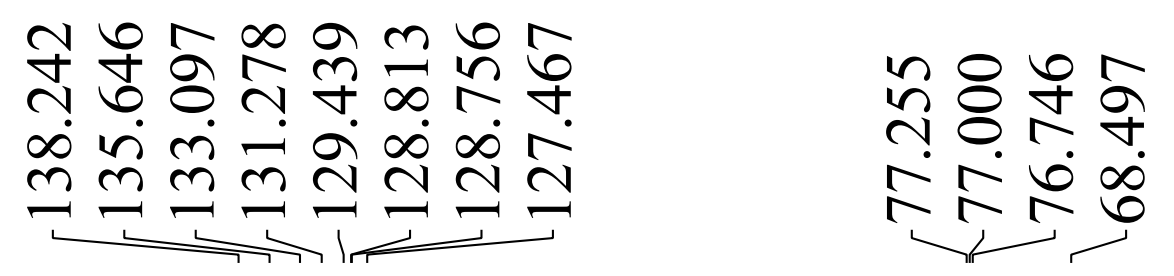

$\stackrel{m}{m}$

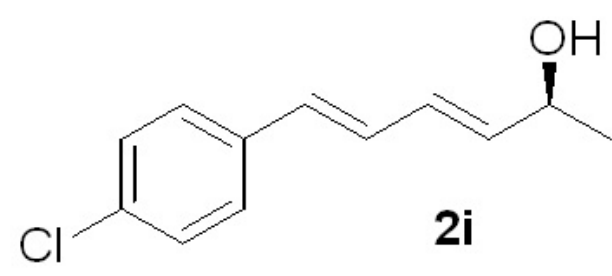

${ }^{13} \mathrm{C}$ NMR $\left(125 \mathrm{MHz}, \mathrm{CDCl}_{3}\right)$

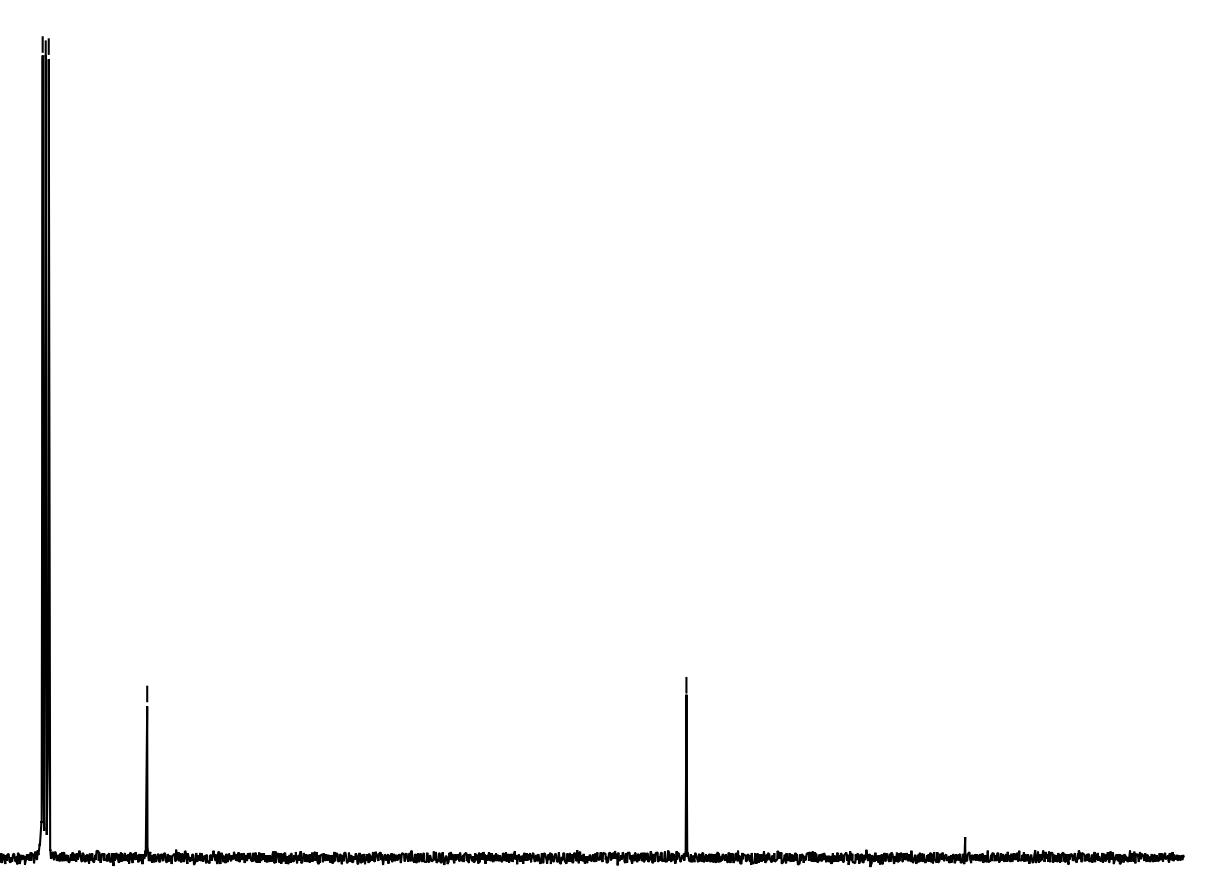

\begin{tabular}{|c|c|c|c|c|c|c|c|c|c|c|}
\hline 210 & 19 & 170 & 150 & 130 & $\begin{array}{l}110 \\
\mathrm{fl}(\mathrm{p}\end{array}$ & $\begin{array}{c}90 \\
\text { om) }\end{array}$ & 70 & 50 & 30 & 10 \\
\hline
\end{tabular}




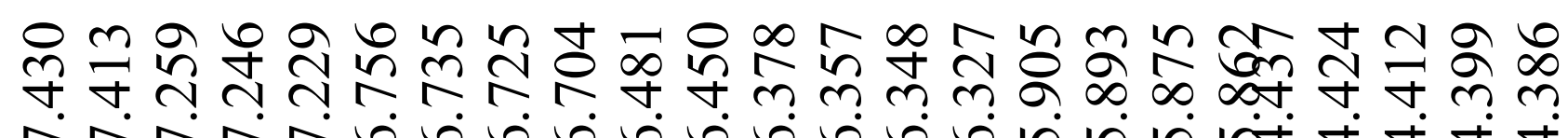
NNN

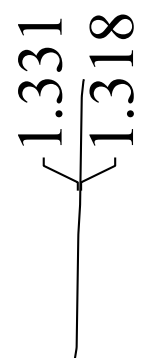

$\oint_{i}$

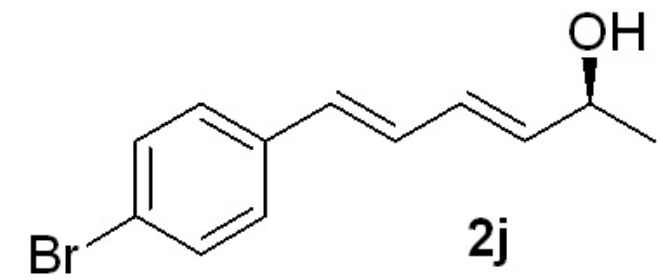

${ }^{1} \mathrm{H} \mathrm{NMR}\left(500 \mathrm{MHz}, \mathrm{CDCl}_{3}\right)$
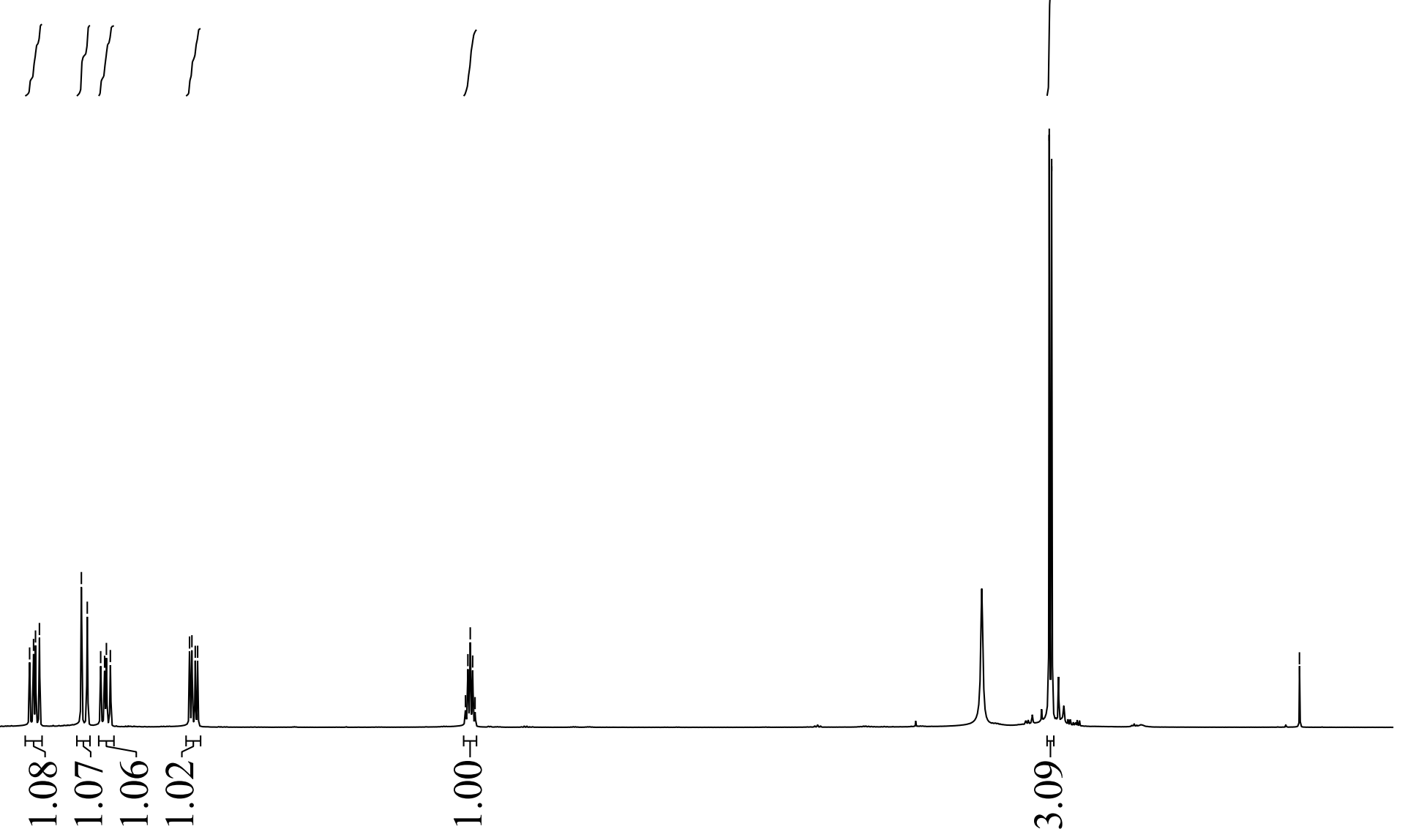

1.5

.5

9.5

9.5

8.5

7.5

6.5

5.5

f1 (ppm) 

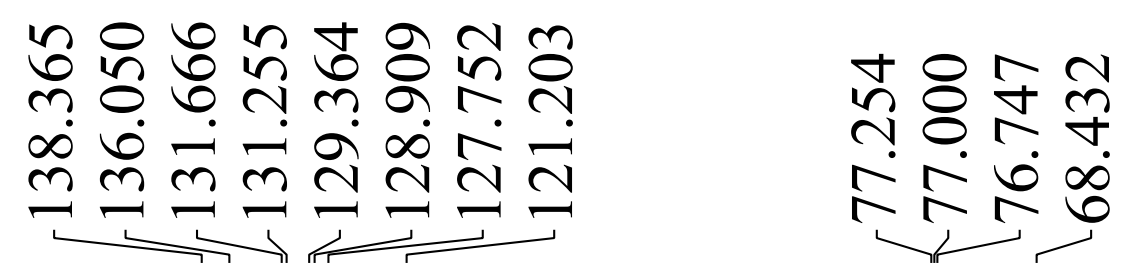

กิ

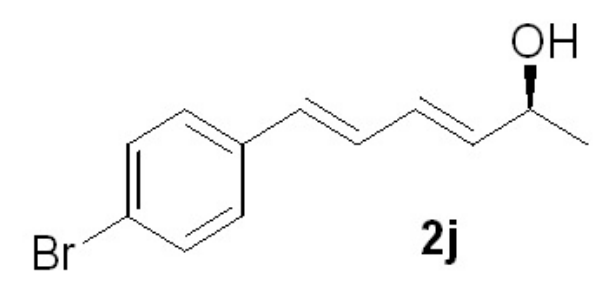

${ }^{13} \mathrm{C} \mathrm{NMR}\left(125 \mathrm{MHz}, \mathrm{CDCl}_{3}\right)$

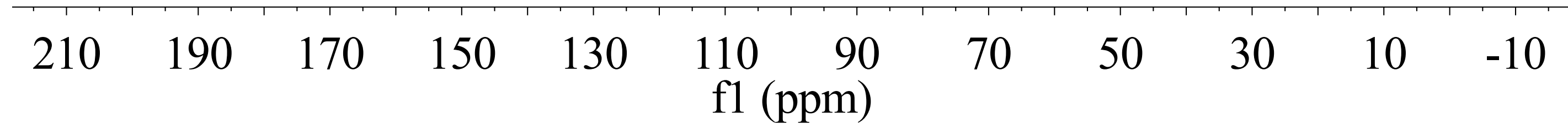




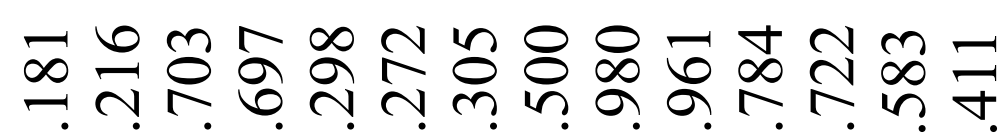

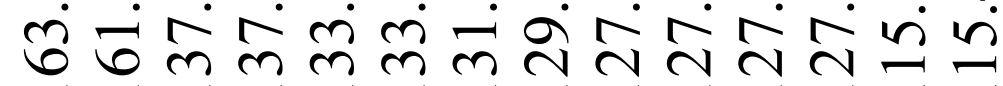

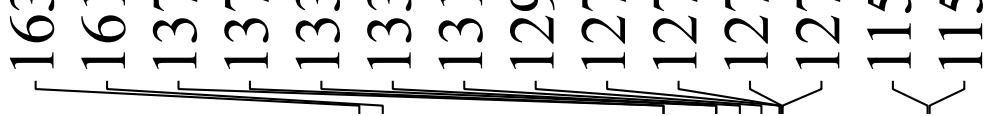

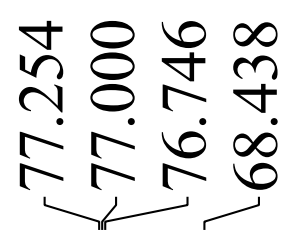

กิ

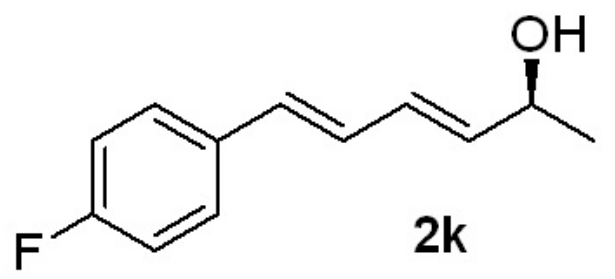

${ }^{13} \mathrm{C} \mathrm{NMR}\left(125 \mathrm{MHz}, \mathrm{CDCl}_{3}\right)$

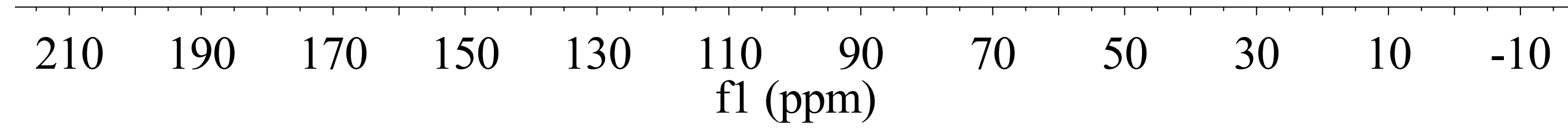




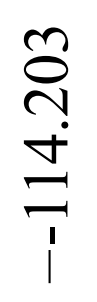

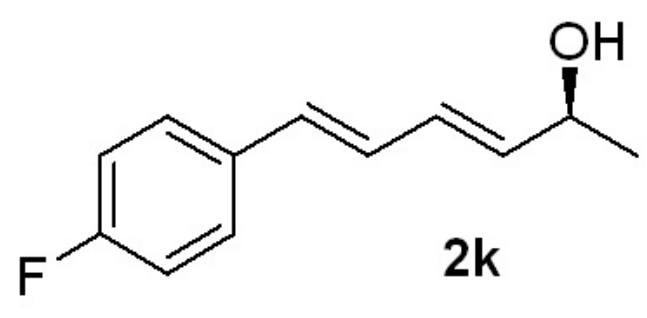

${ }^{19} \mathrm{~F}$ NMR $\left(471 \mathrm{MHz}, \mathrm{CDCl}_{3}\right)$

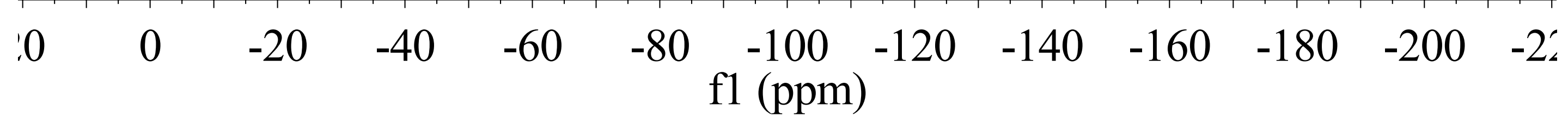




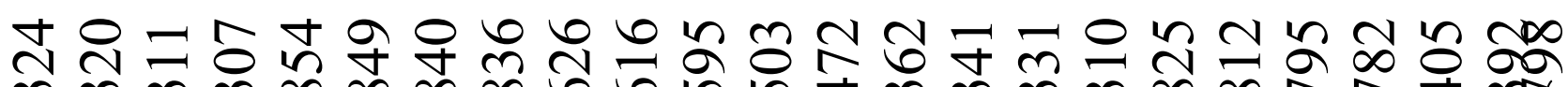

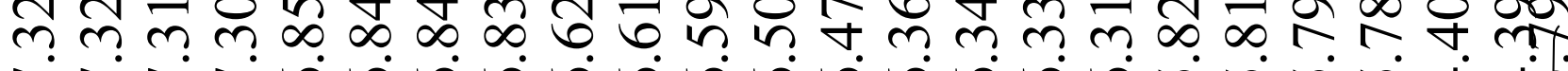
NN $N$.
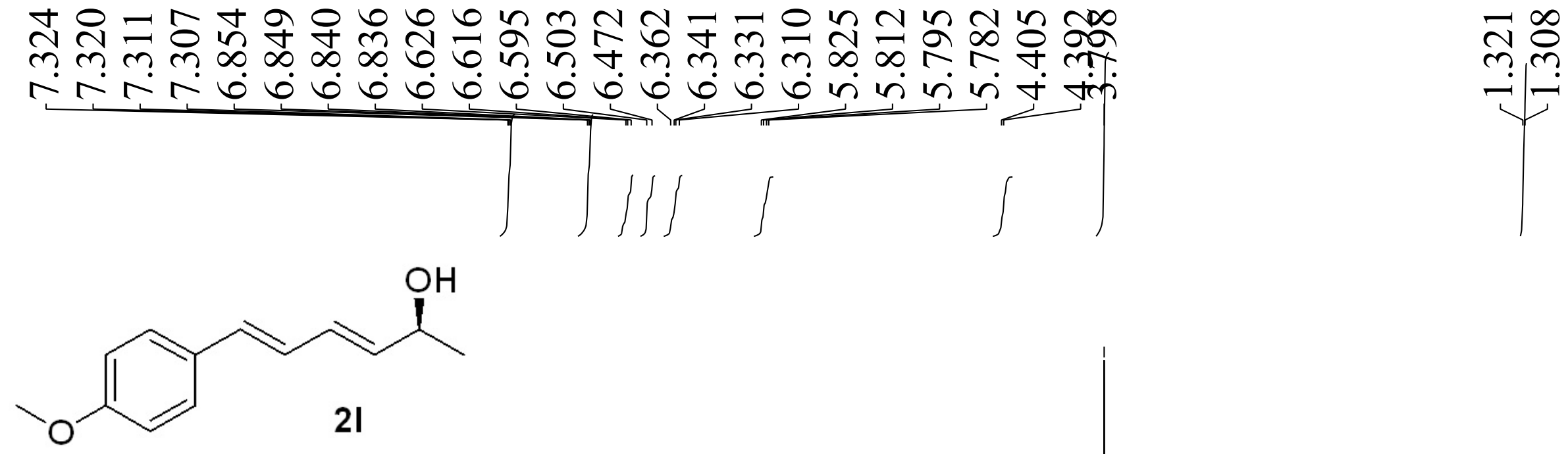

${ }^{1} \mathrm{H}$ NMR $\left(500 \mathrm{MHz}, \mathrm{CDCl}_{3}\right)$
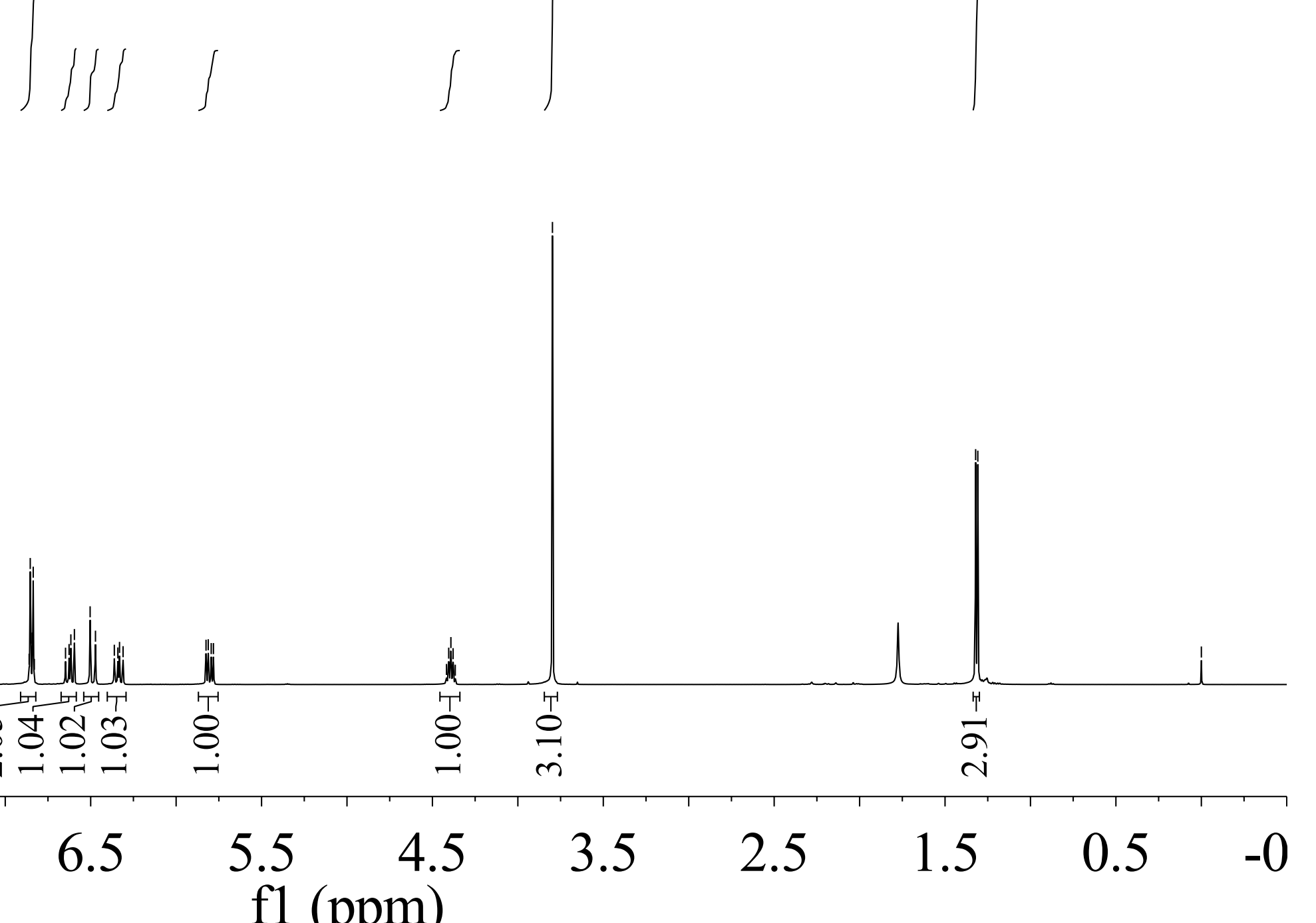

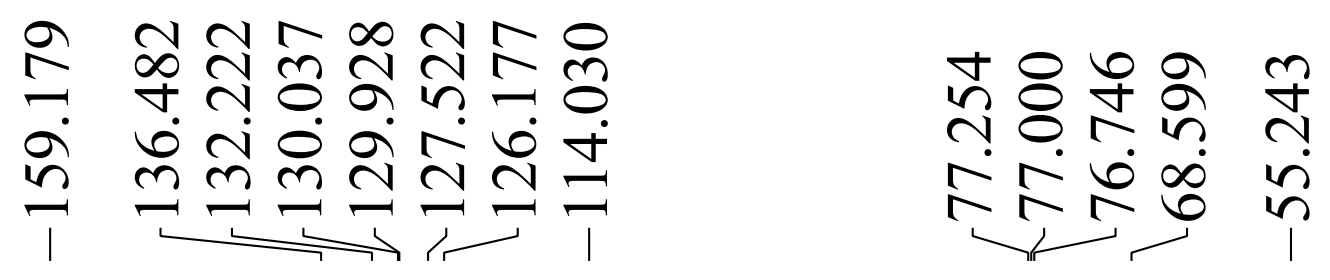

$N$
$\cdots$
$\cdots$

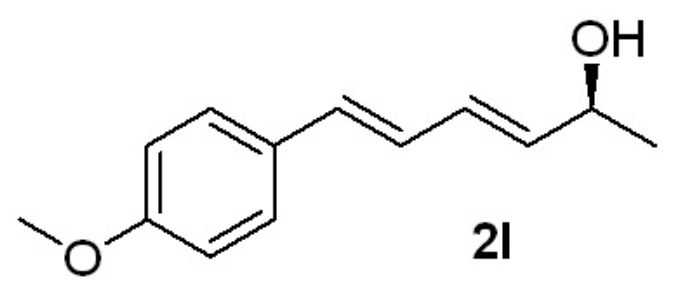

${ }^{13} \mathrm{C}$ NMR $\left(125 \mathrm{MHz}, \mathrm{CDCl}_{3}\right)$

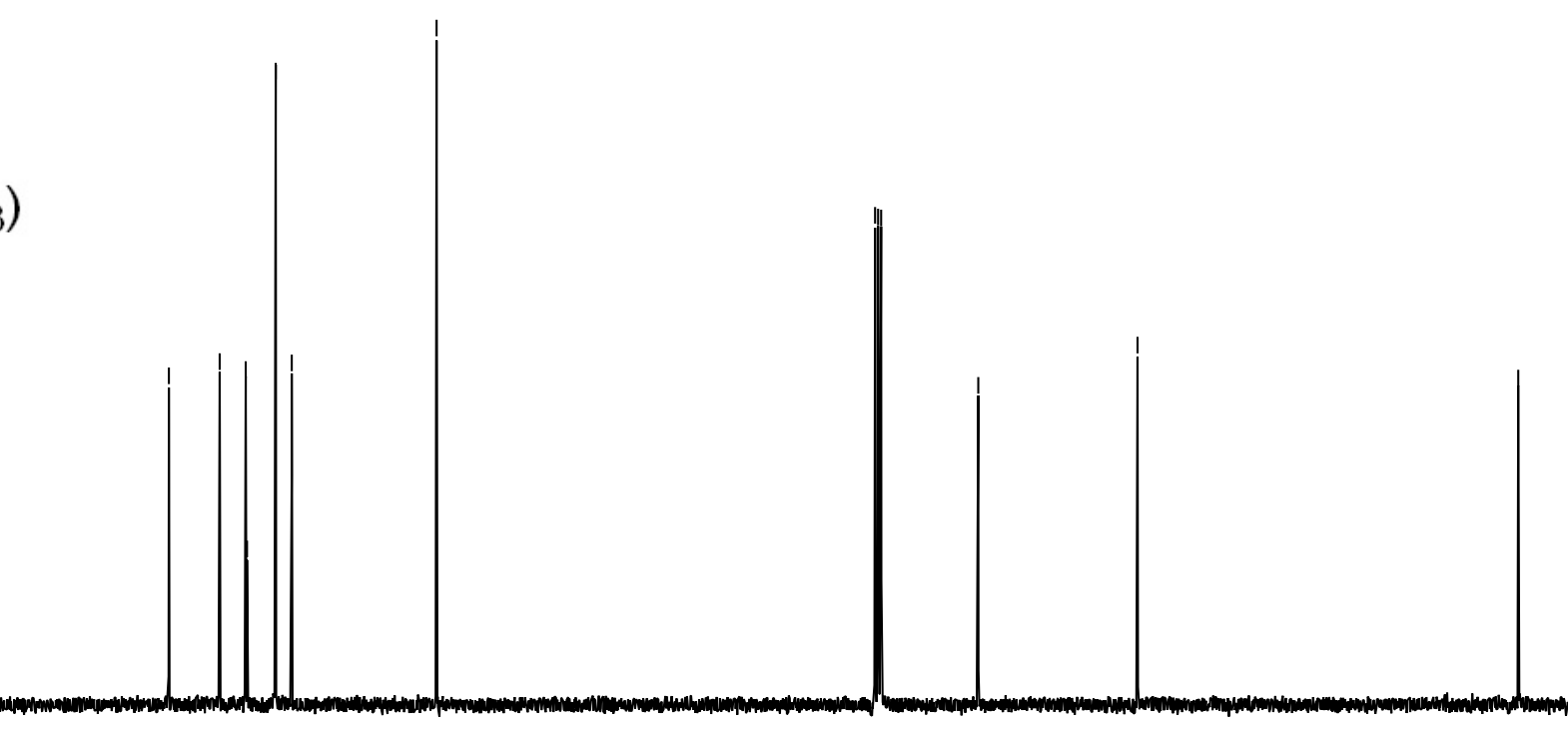

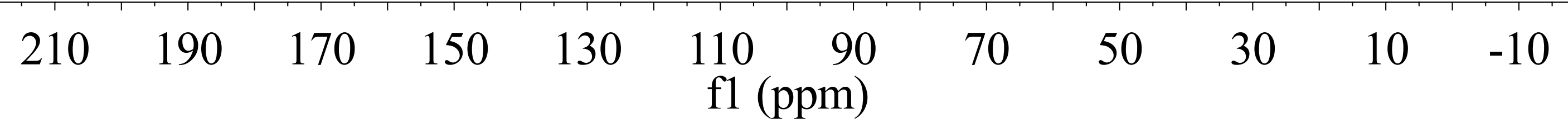




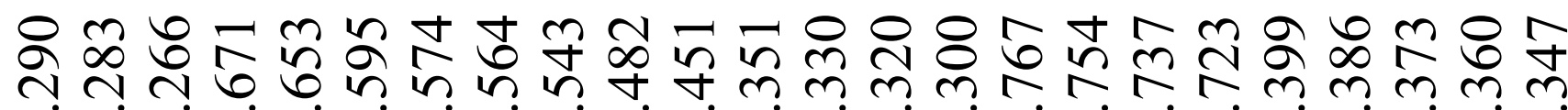

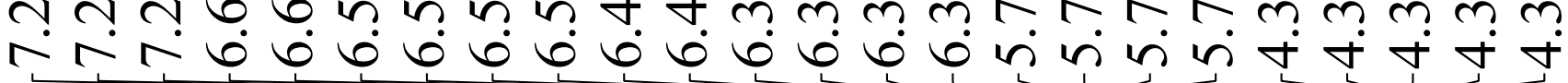
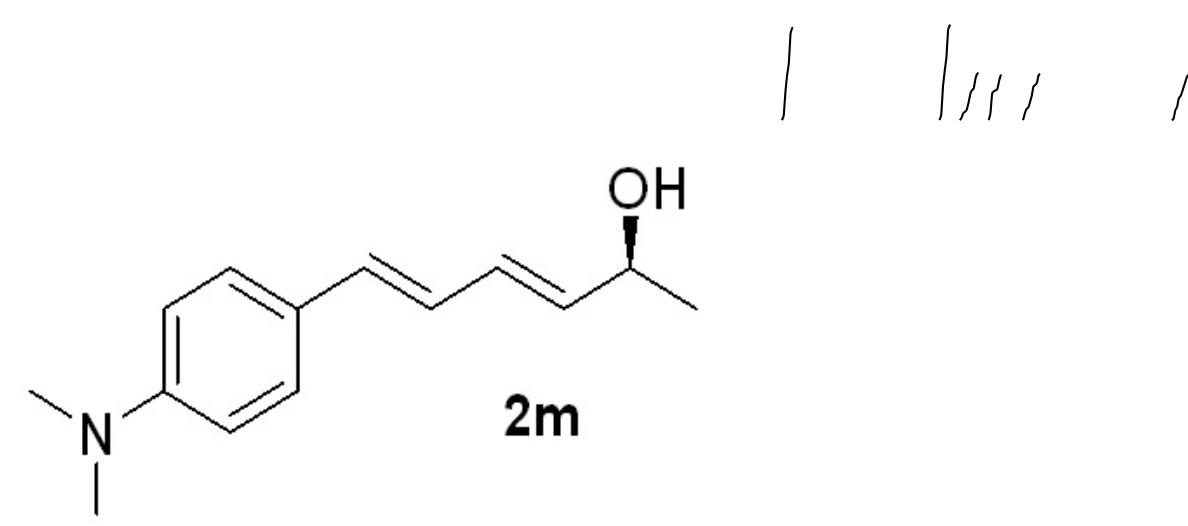

${ }^{1} \mathrm{H}$ NMR $\left(500 \mathrm{MHz}, \mathrm{CDCl}_{3}\right)$

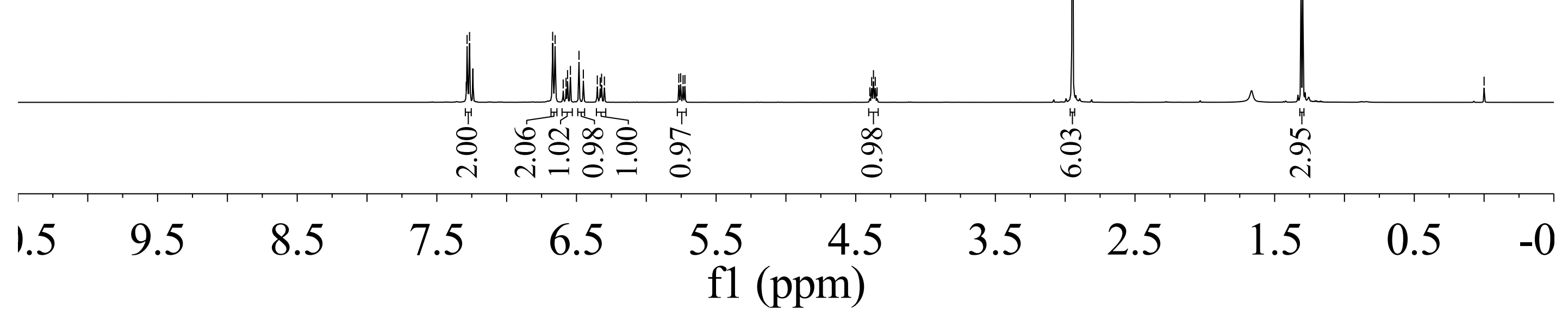



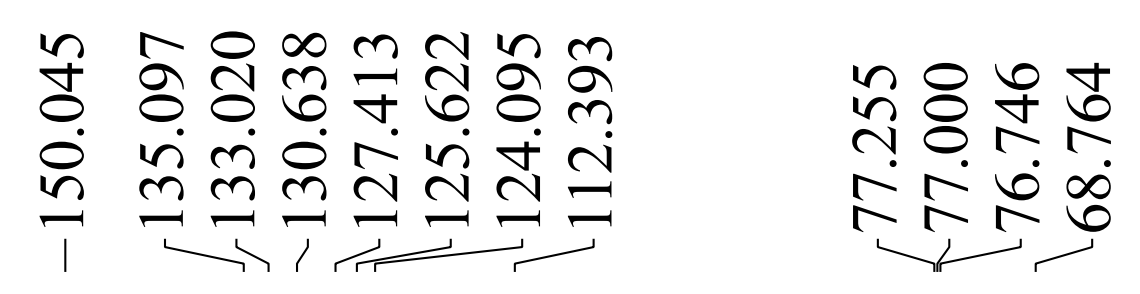

$\begin{array}{ll}\stackrel{m}{+} & \stackrel{n}{+} \\ \stackrel{+}{+} & \stackrel{1}{1}\end{array}$

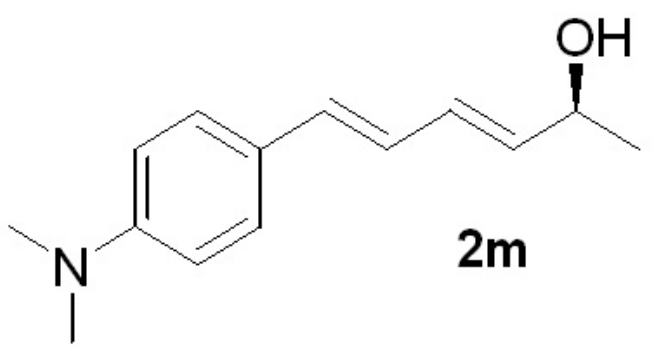

${ }^{13} \mathrm{C}$ NMR (125 MHz, $\left.\mathrm{CDCl}_{3}\right)$

\begin{tabular}{|c|c|c|c|c|c|c|c|c|c|}
\hline 210 & 190 & 170 & 150 & 130 & $\begin{array}{c}110 \quad 90 \\
\mathrm{fl}(\mathrm{ppm})\end{array}$ & 70 & 50 & 30 & 10 \\
\hline
\end{tabular}


$\infty$
$-\infty$ ए
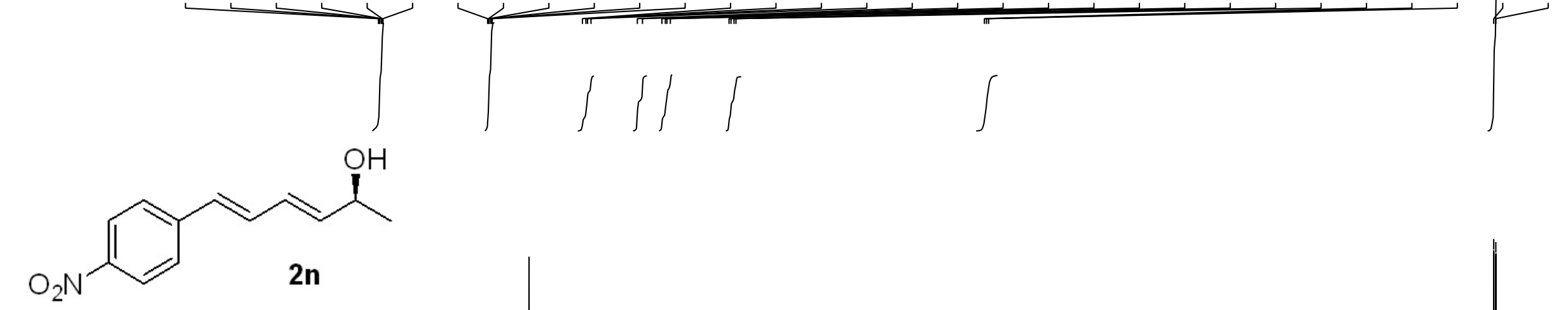

${ }^{1} \mathrm{HNMR}\left(500 \mathrm{MHz}, \mathrm{CDCl}_{3}\right)$

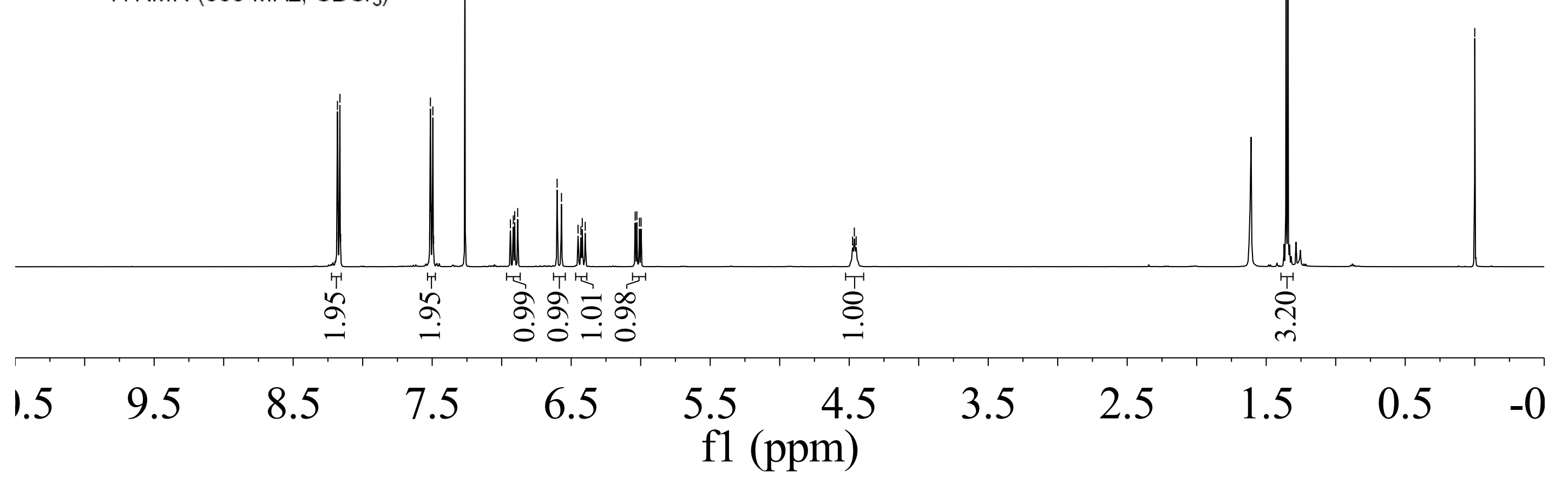




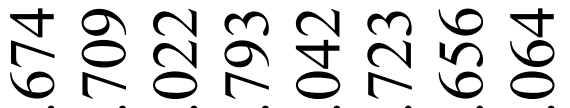

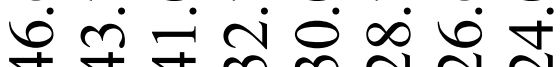

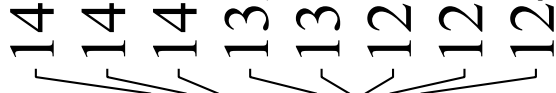

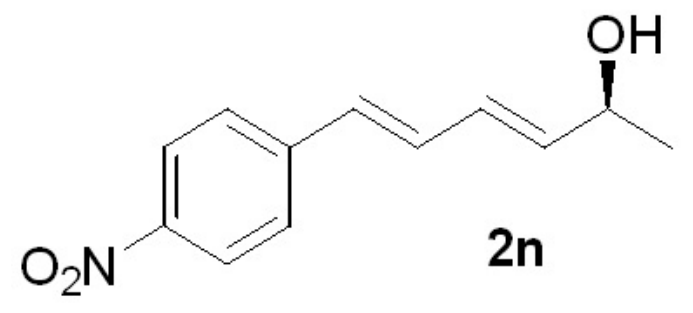

${ }^{13} \mathrm{C}$ NMR $\left(125 \mathrm{MHz}, \mathrm{CDCl}_{3}\right)$

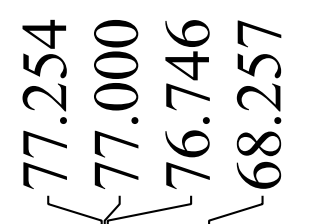

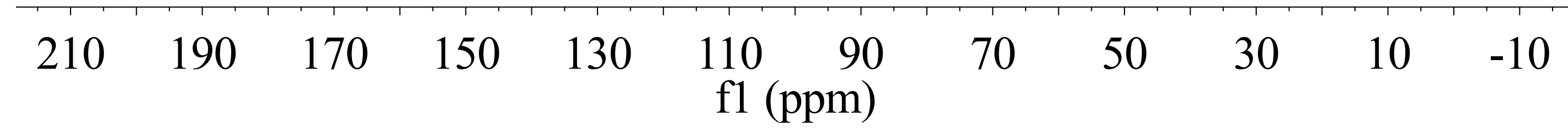




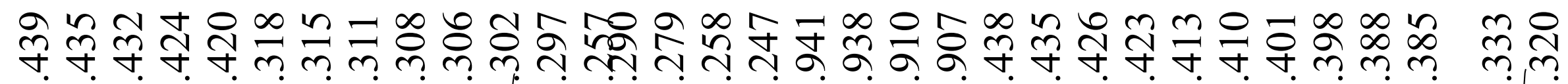

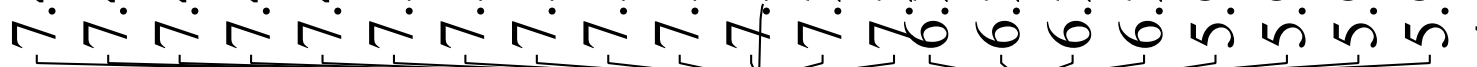
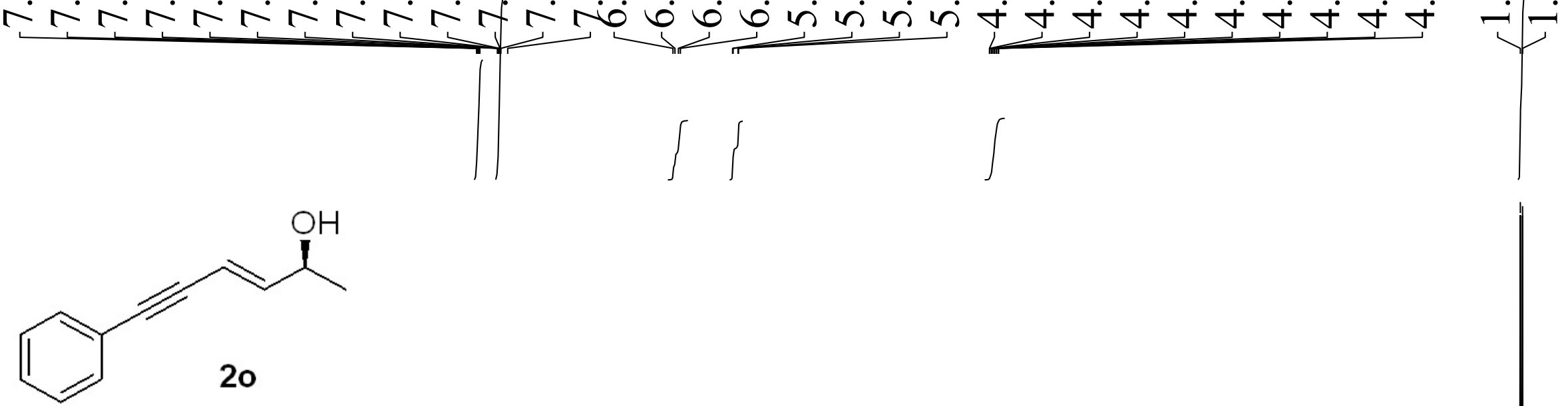

$\mathrm{HNMR}\left(500 \mathrm{MHz}, \mathrm{CDCl}_{3}\right)$

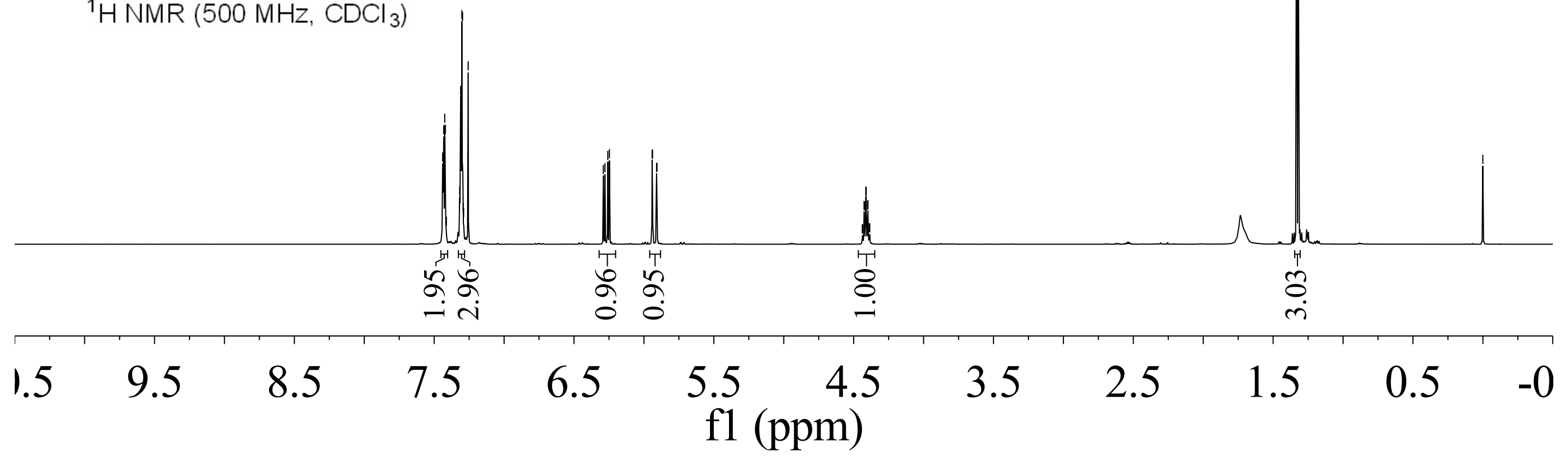




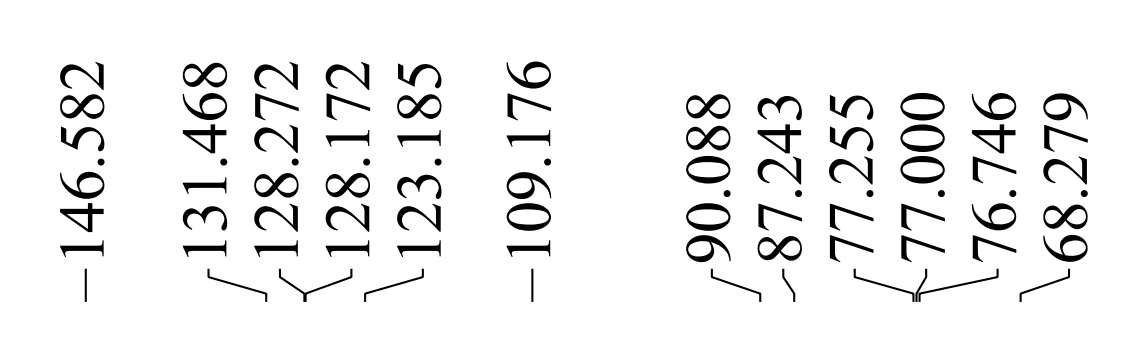

å

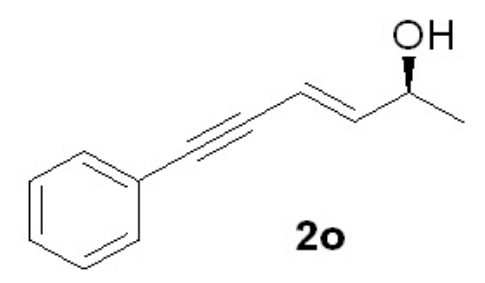

${ }^{13} \mathrm{C} \mathrm{NMR}\left(125 \mathrm{MHz}, \mathrm{CDCl}_{3}\right)$

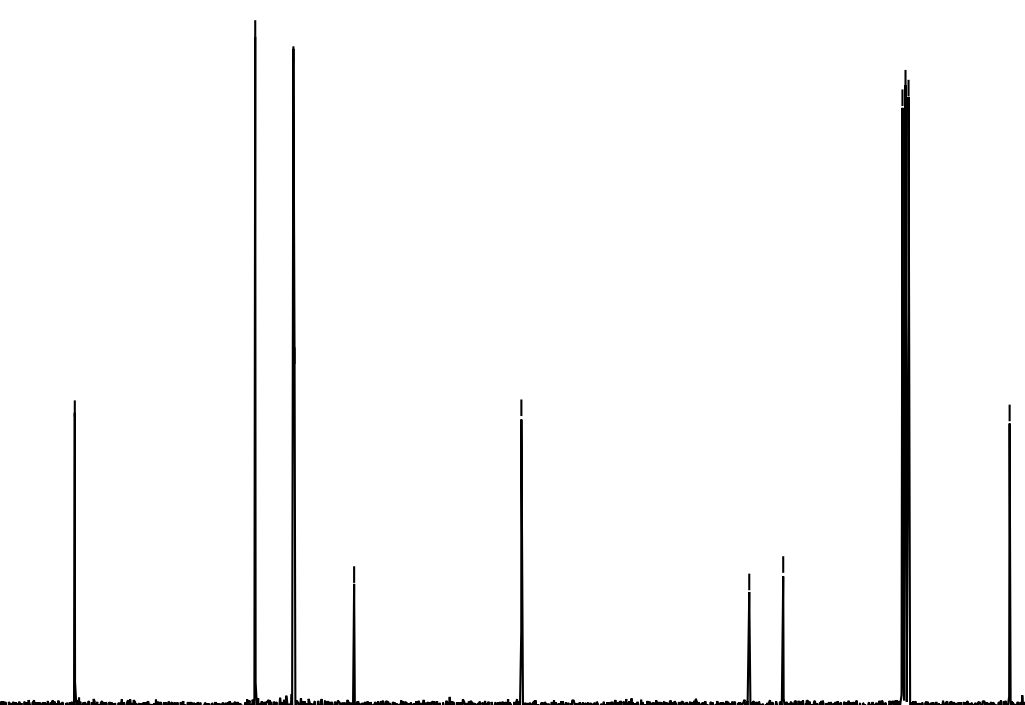

\begin{tabular}{|c|c|c|c|c|c|c|c|c|c|c|}
\hline 210 & 190 & 170 & 150 & 130 & 110 & $\begin{array}{l}90 \\
\mathrm{~m})\end{array}$ & 70 & 50 & 30 & 10 \\
\hline
\end{tabular}




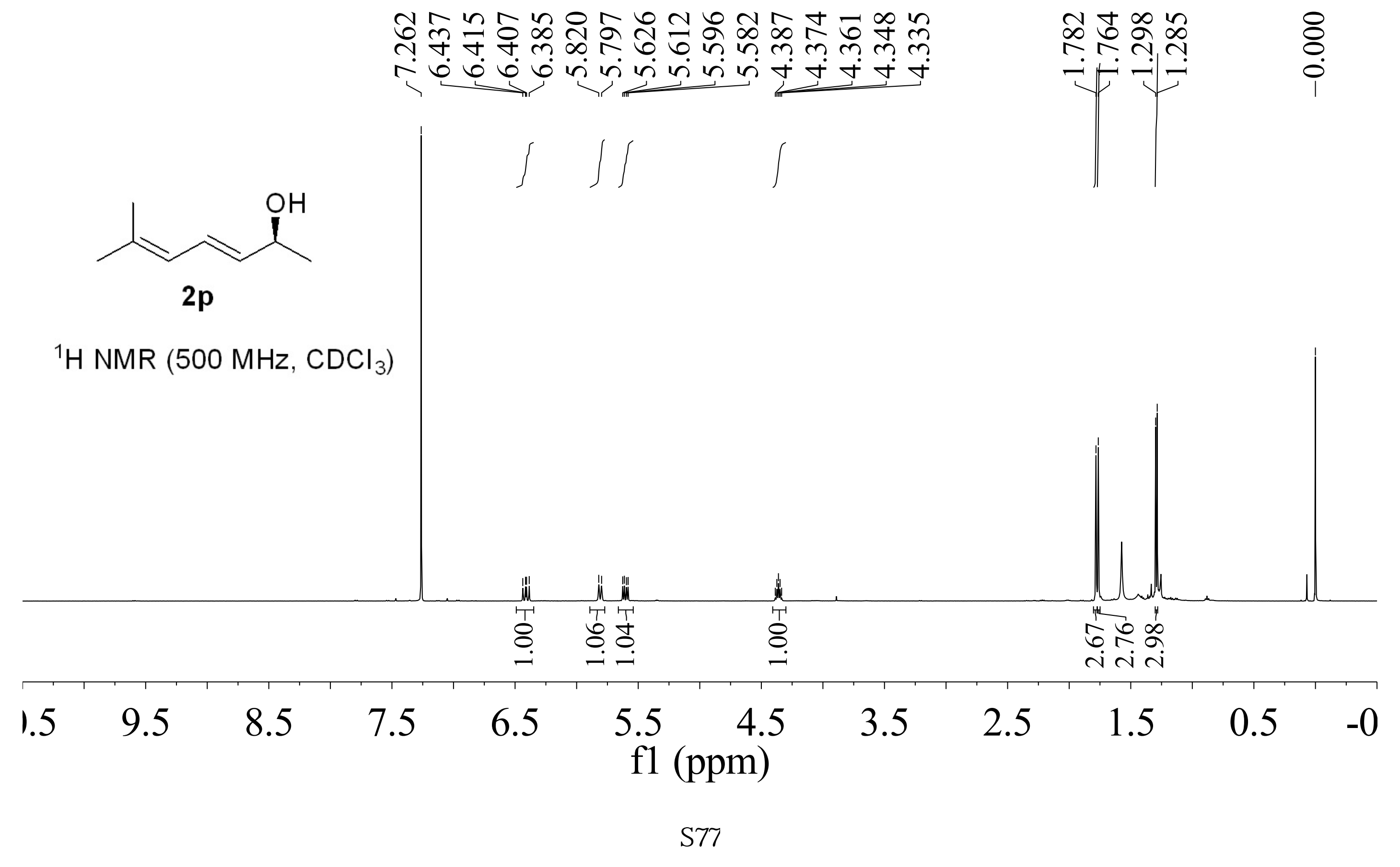



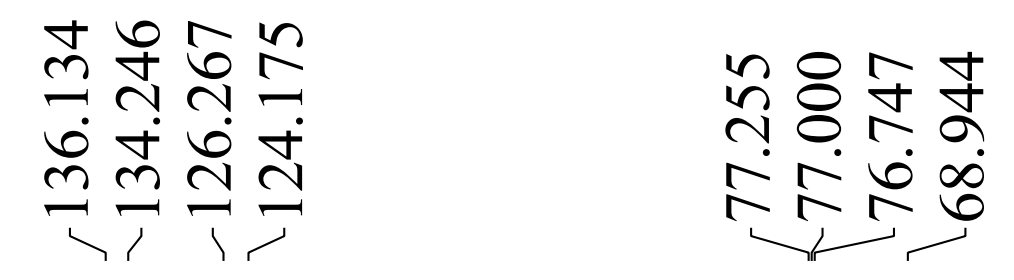

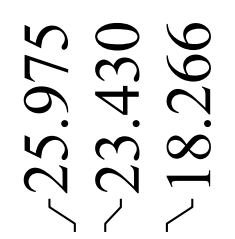

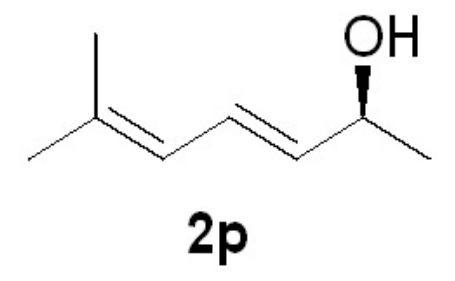

${ }^{13} \mathrm{C}$ NMR $\left(125 \mathrm{MHz}, \mathrm{CDCl}_{3}\right)$

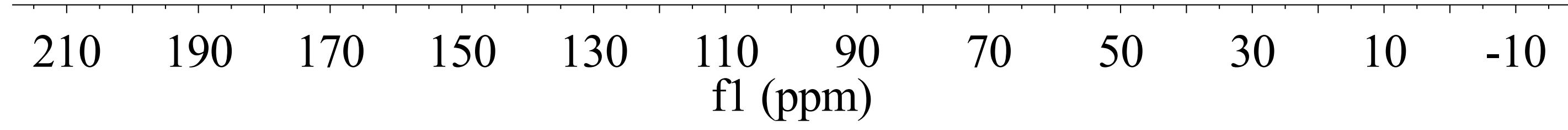



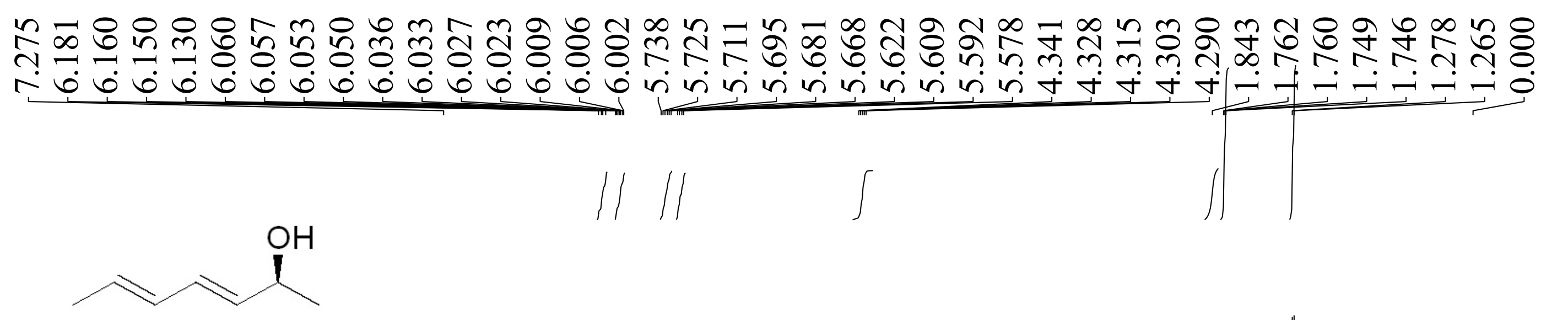

2q

${ }^{1} \mathrm{H}$ NMR $\left(500 \mathrm{MHz}, \mathrm{CDCl}_{3}\right)$

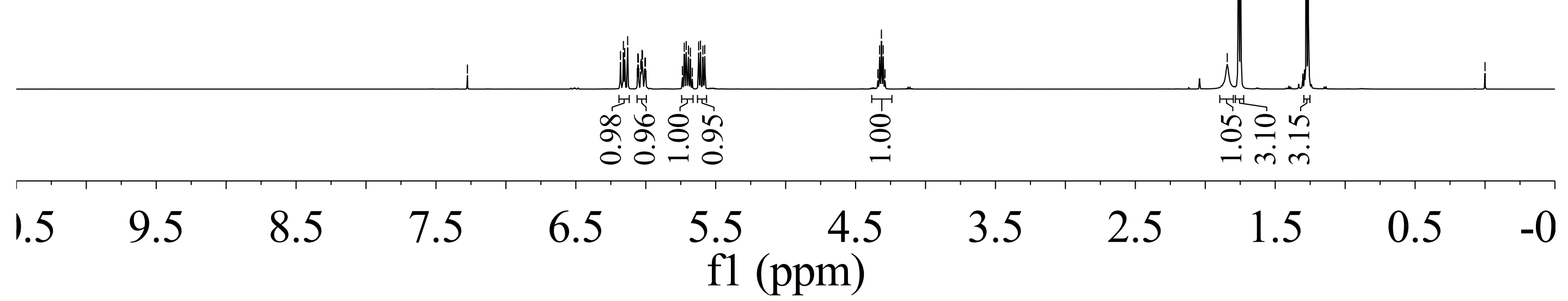




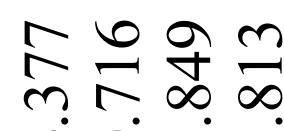

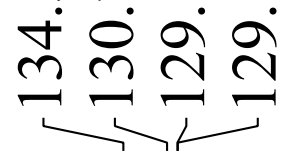

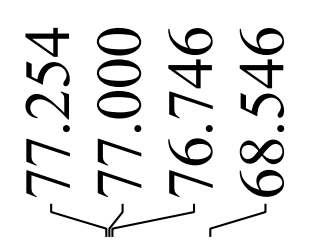

กิ

m $\infty$

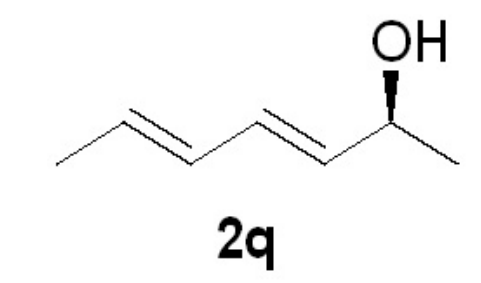

${ }^{13} \mathrm{C}$ NMR $\left(125 \mathrm{MHz}, \mathrm{CDCl}_{3}\right)$

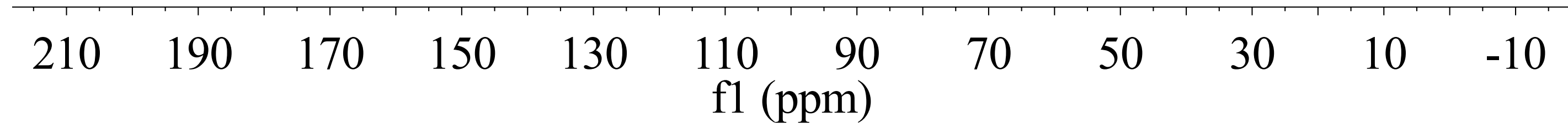




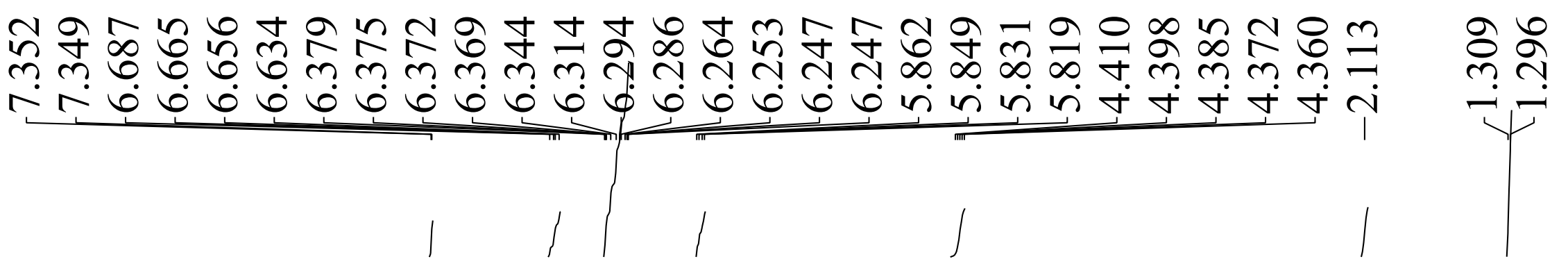

㐔

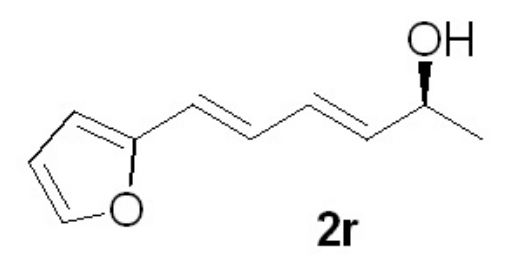

${ }^{1} \mathrm{H}$ NMR (500 MHz, $\mathrm{CDCl}_{3}$ )

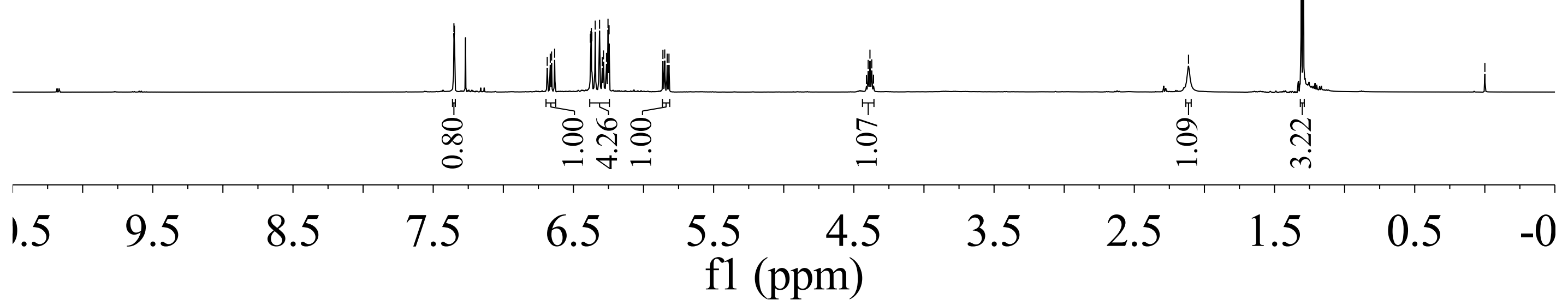



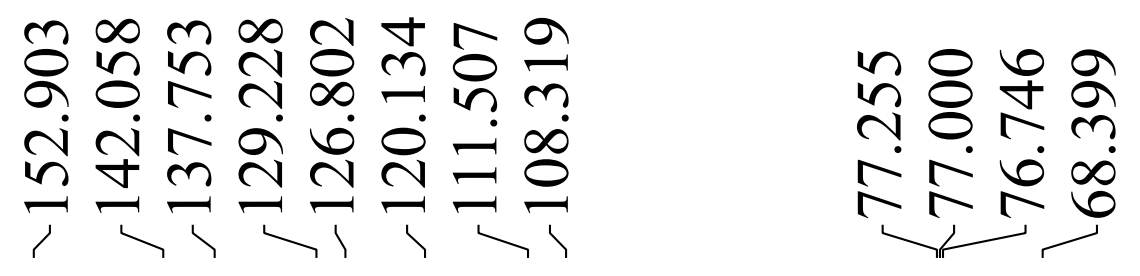

$\frac{0}{2}$

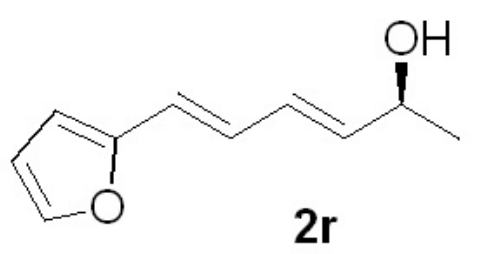

${ }^{13} \mathrm{C}$ NMR $\left(125 \mathrm{MHz}, \mathrm{CDCl}_{3}\right)$

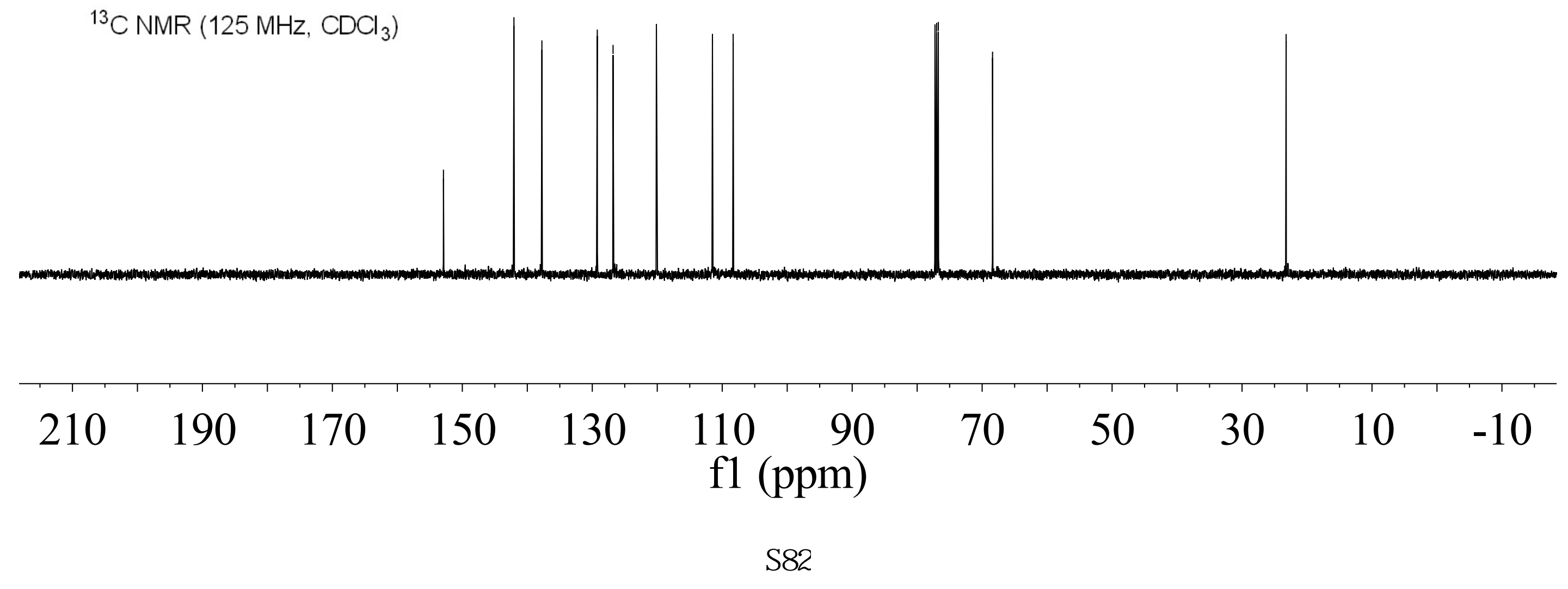




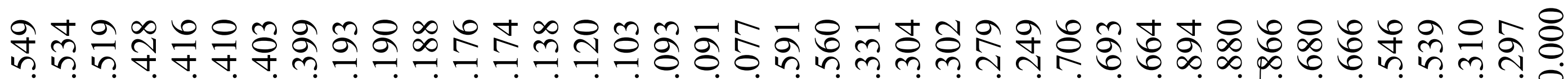

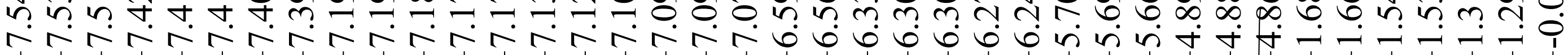
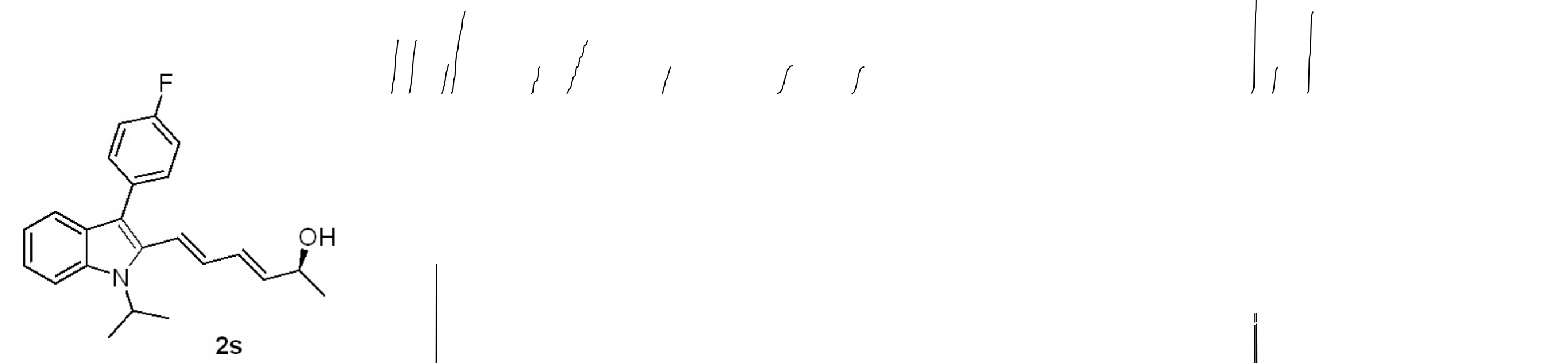

${ }^{1} \mathrm{H} \mathrm{NMR}\left(500 \mathrm{MHz}, \mathrm{CDCl}_{3}\right)$

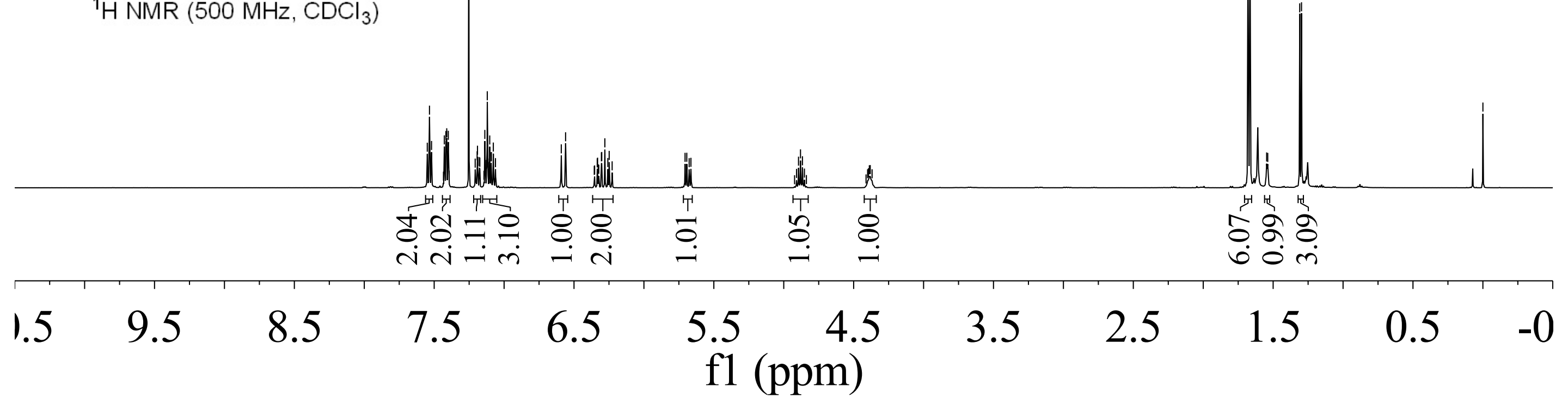




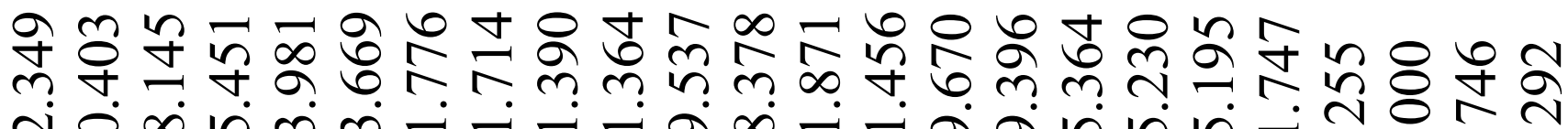

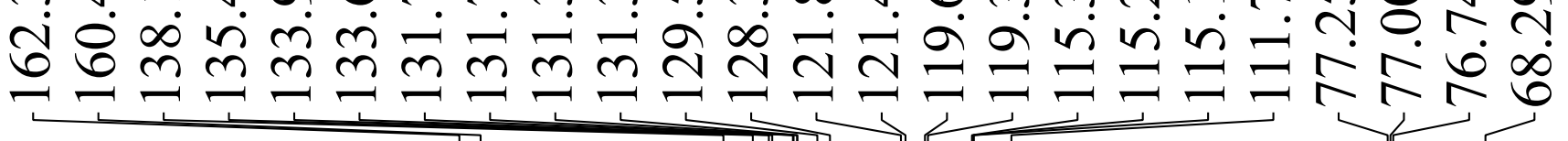
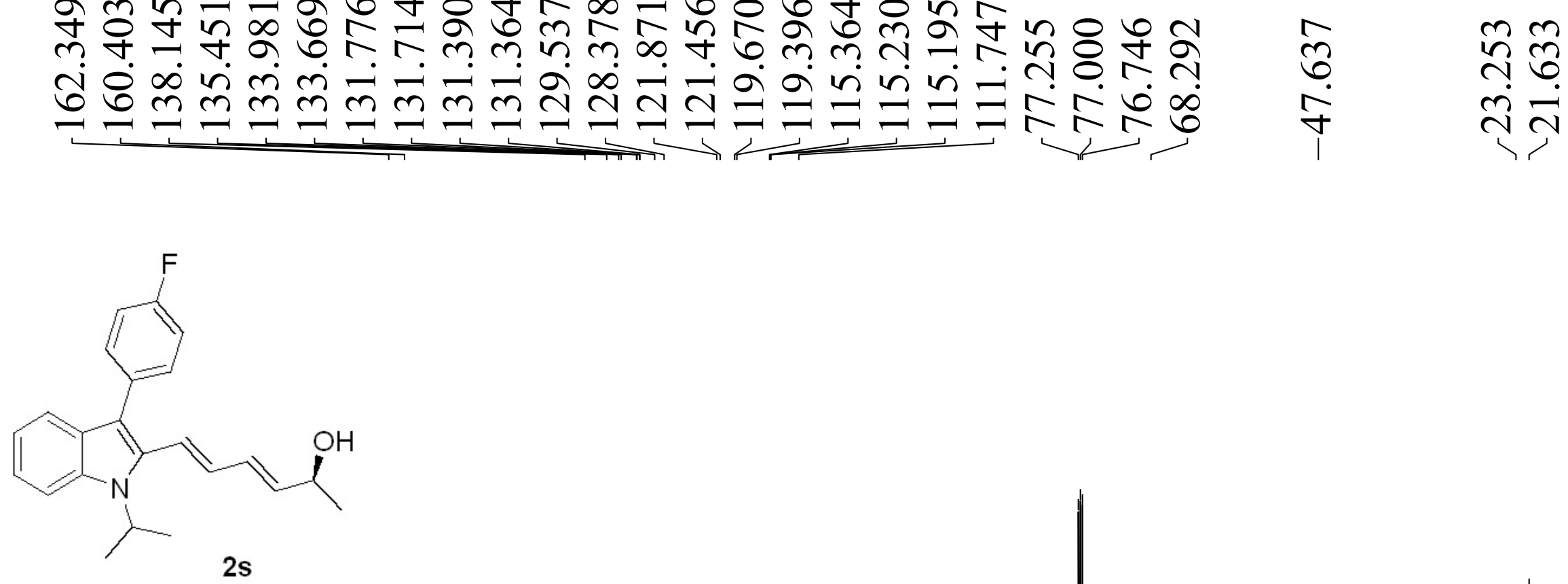

${ }^{13} \mathrm{C} \mathrm{NMR}\left(125 \mathrm{MHz}, \mathrm{CDCl}_{3}\right)$

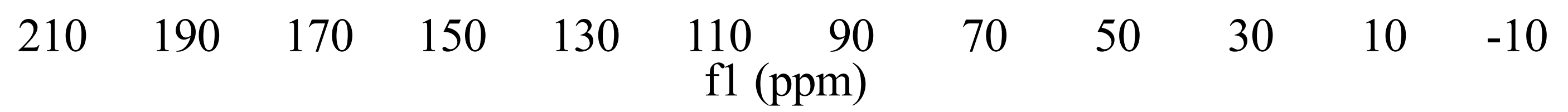



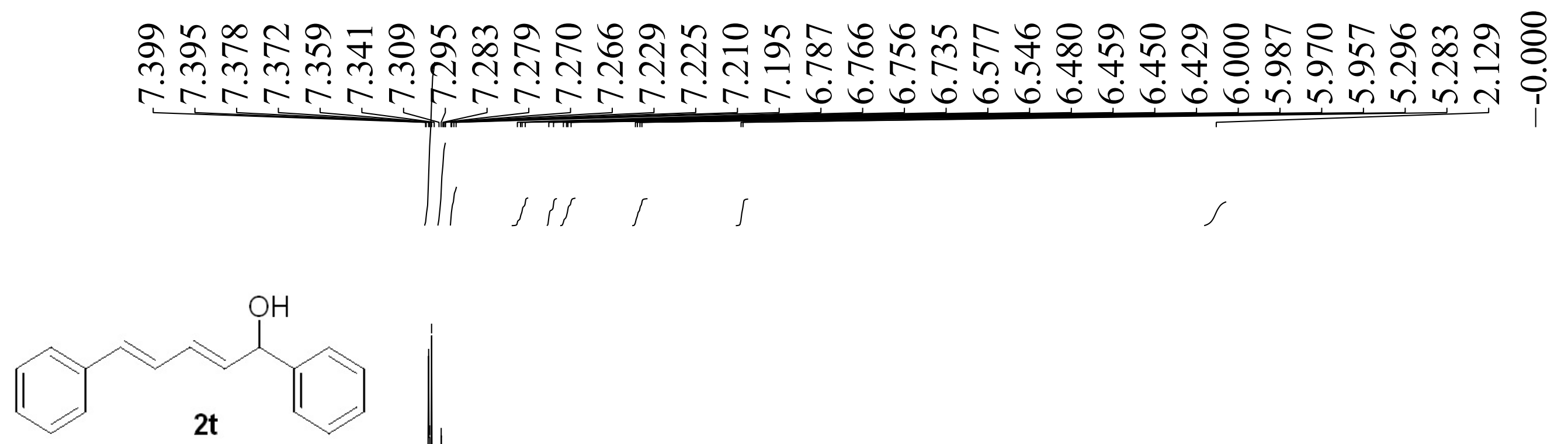

${ }^{1} \mathrm{H}$ NMR $\left(500 \mathrm{MHz}, \mathrm{CDCl}_{3}\right)$

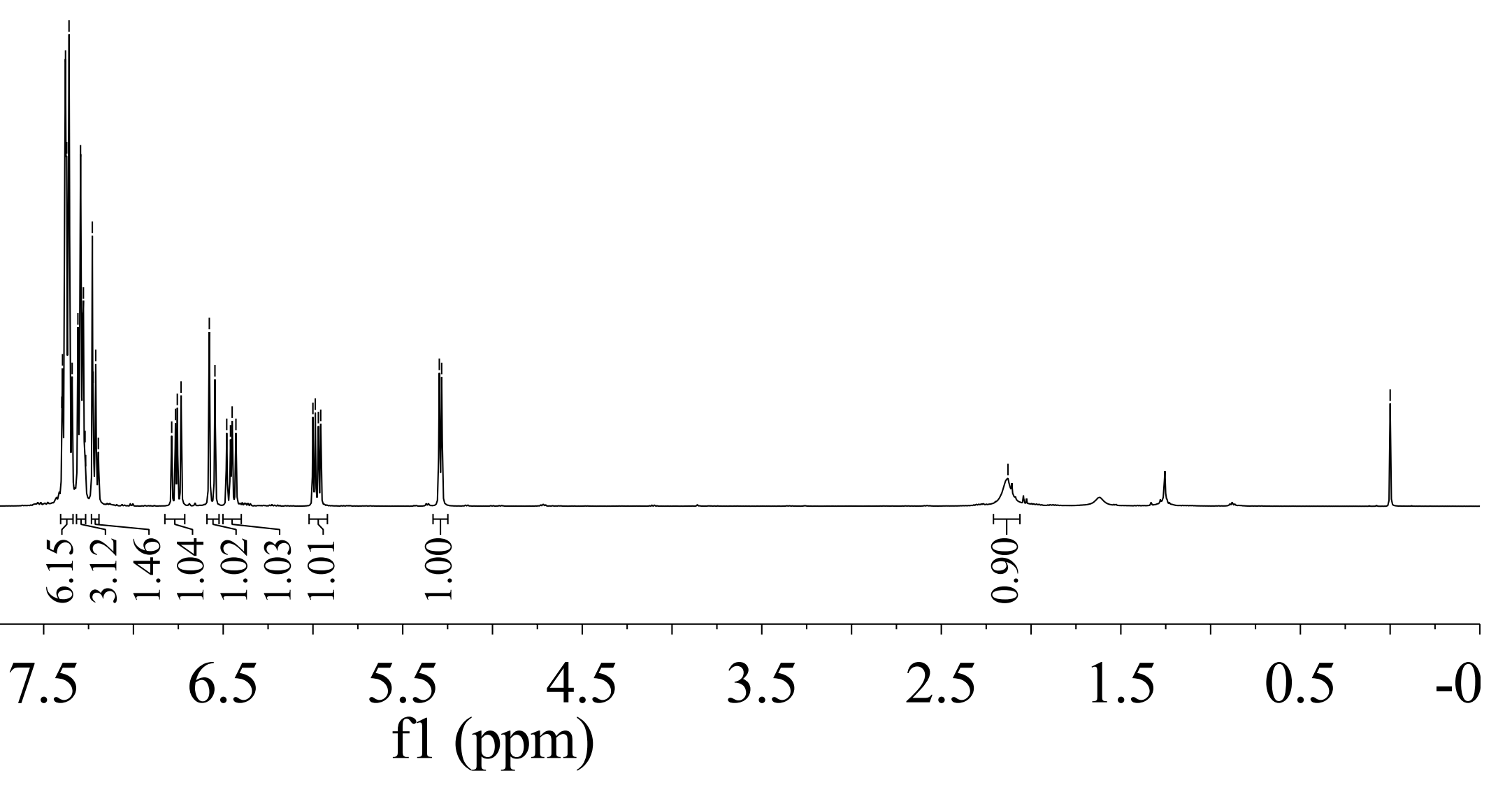



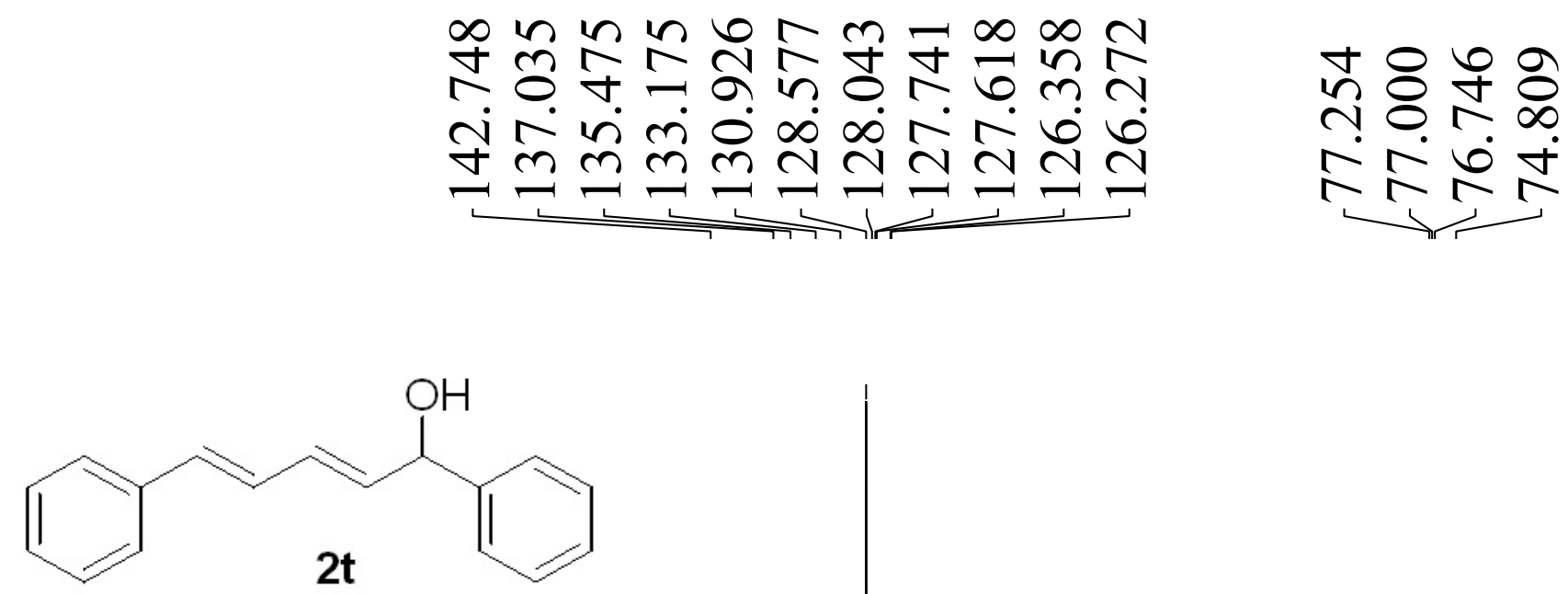

${ }^{13} \mathrm{C}$ NMR $\left(125 \mathrm{MHz}, \mathrm{CDCl}_{3}\right)$

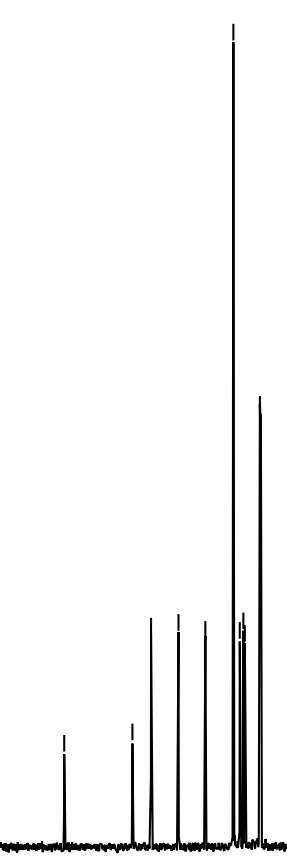

\begin{tabular}{|c|c|c|c|c|c|c|c|c|c|c|}
\hline 210 & 190 & 170 & 150 & 130 & 110 & $\begin{array}{c}90 \\
\text { om) }\end{array}$ & 70 & 50 & 30 & 10 \\
\hline
\end{tabular}




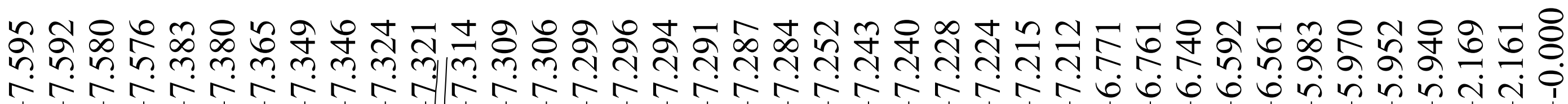
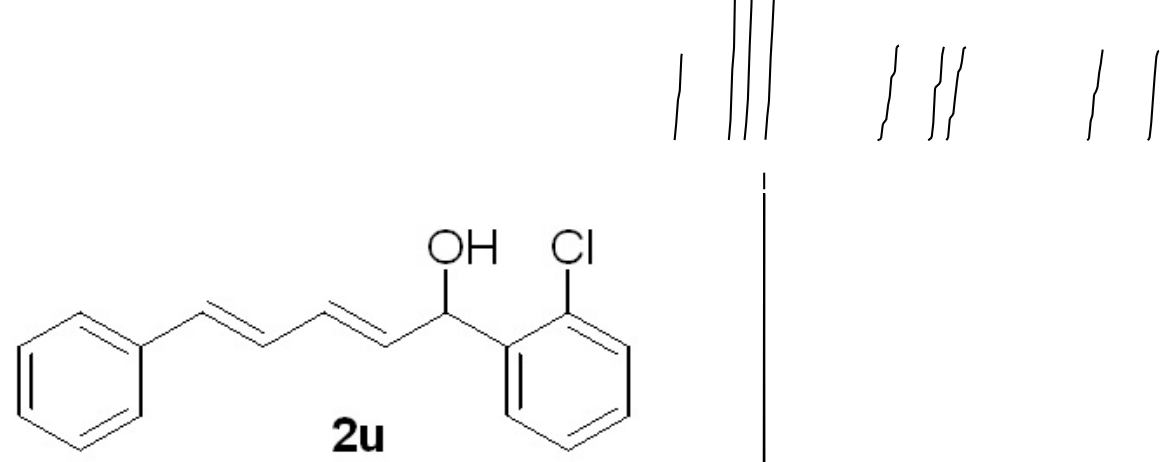

${ }^{1} \mathrm{H}$ NMR $\left(500 \mathrm{MHz}, \mathrm{CDCl}_{3}\right)$

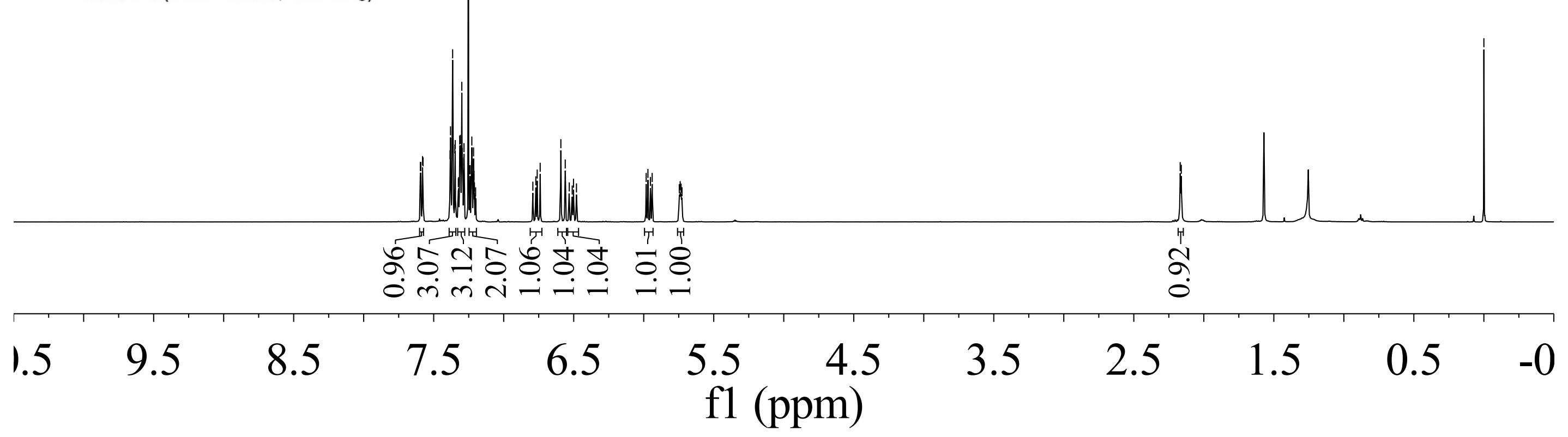




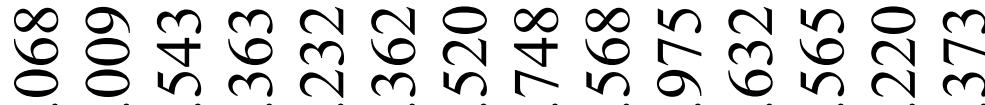 $0 \mathrm{~m} m \mathrm{~N}-\mathrm{i} \infty \mathbf{N} \mathrm{N} N$

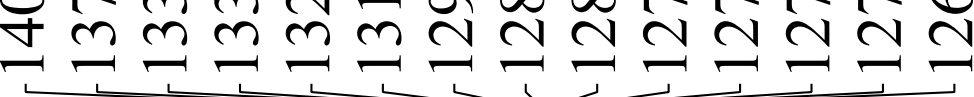

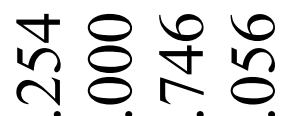 \\ N

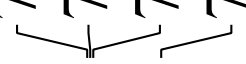

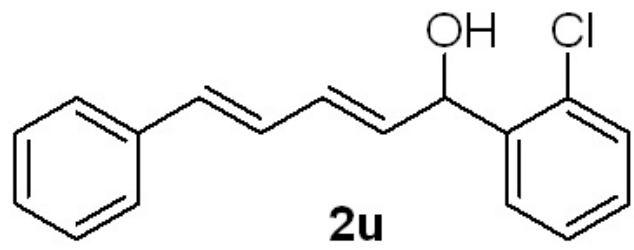

${ }^{13} \mathrm{C} \mathrm{NMR}\left(125 \mathrm{MHz}, \mathrm{CDCl}_{3}\right)$

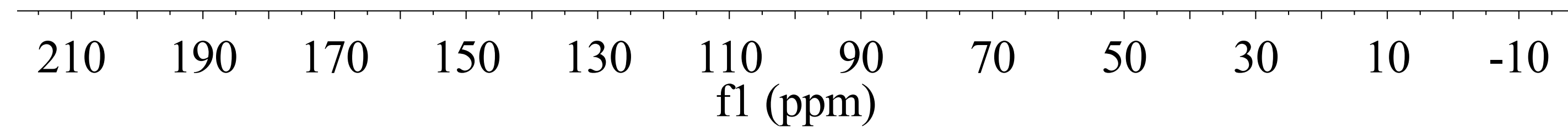


<smiles>CC(O)/C=C/C=C/c1ccccc1</smiles>

racemate for $\mathbf{2 a}$

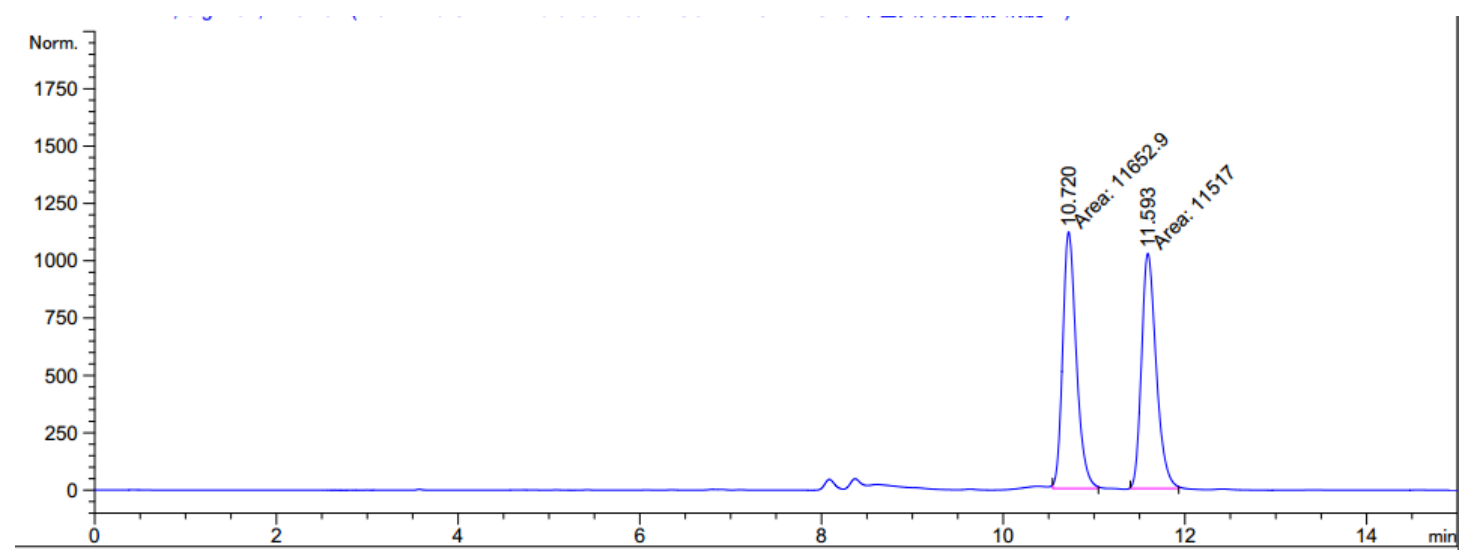

Signal 1: DAD1 B, Sig=254,4 Ref=off

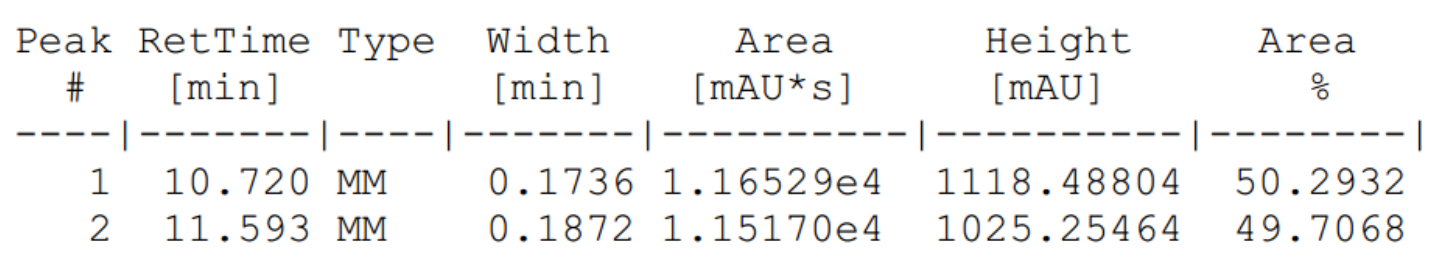<smiles>CC(O)C=CC=Cc1ccccc1</smiles>

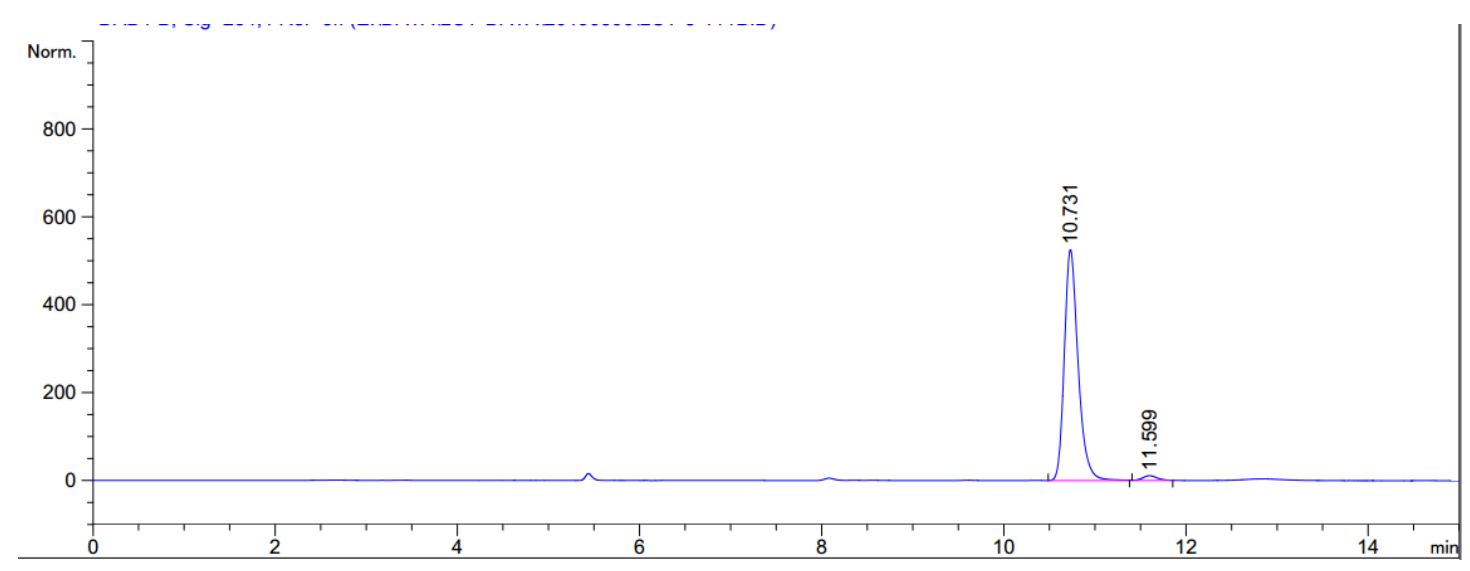

Signal 1: DAD1 B, Sig=254,4 Ref=off

\begin{tabular}{|c|c|c|c|c|c|c|}
\hline $\begin{array}{c}\text { Peak } \\
\#\end{array}$ & $\begin{array}{c}\text { RetTime } \\
\text { [min] }\end{array}$ & Type & $\begin{array}{l}\text { Width } \\
\text { [min] }\end{array}$ & $\begin{array}{c}\text { Area } \\
{\left[\mathrm{mAU}^{\star} \mathrm{s}\right]}\end{array}$ & $\begin{array}{l}\text { Height } \\
{[\mathrm{mAU}]}\end{array}$ & $\begin{array}{c}\text { Area } \\
\frac{\circ}{0}\end{array}$ \\
\hline-1 & 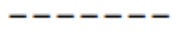 & & - & --- & -- & 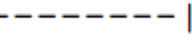 \\
\hline 1 & 10.731 & BV & 0.1588 & 5530.25098 & 525.35400 & 8115 \\
\hline 2 & 11.599 & VV & 0.1370 & 123.73991 & 10.97841 & 2.1885 \\
\hline
\end{tabular}


<smiles>CCCC(O)/C=C/C=C/c1ccccc1</smiles>

racemate for $\mathbf{2 b}$

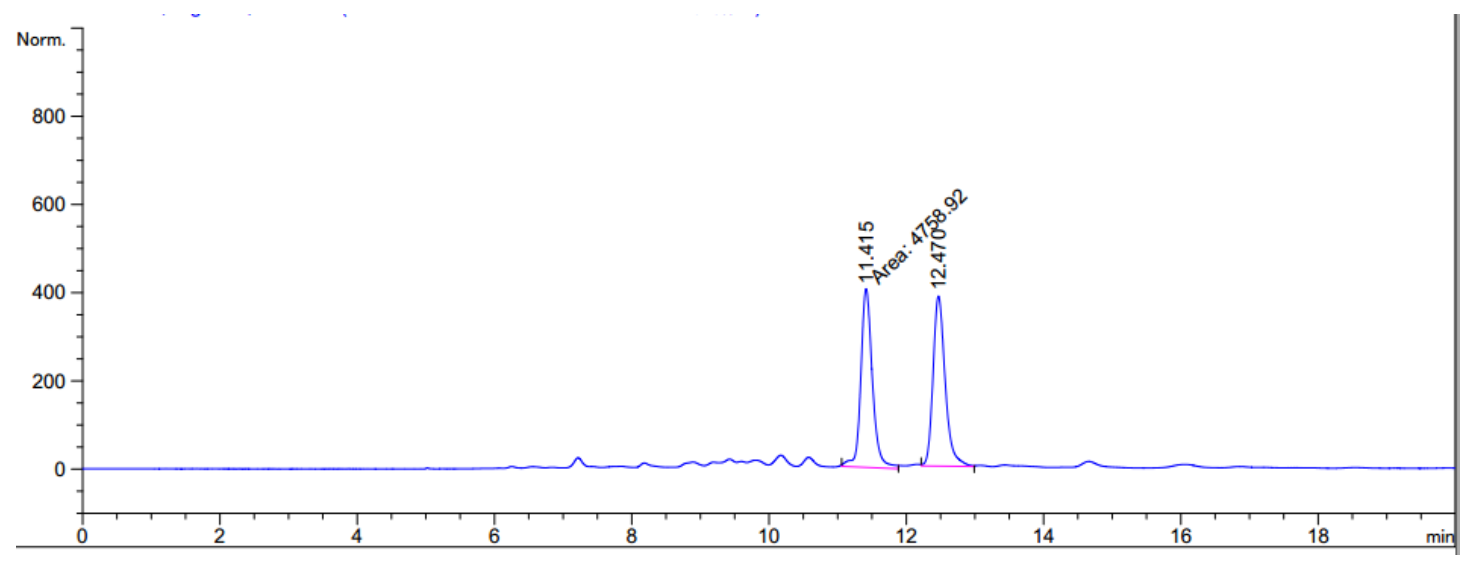

Signal 1: DAD1 B, Sig=254,4 Ref=off

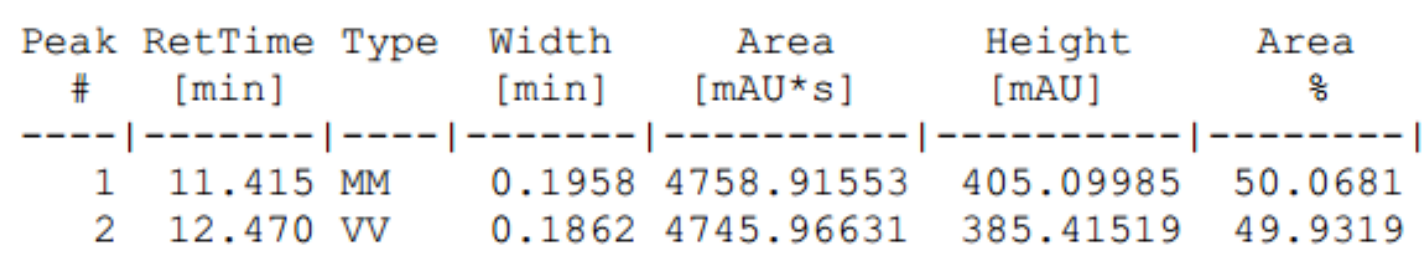<smiles>CCC[C@H](O)/C=C/C=C/c1ccccc1</smiles>

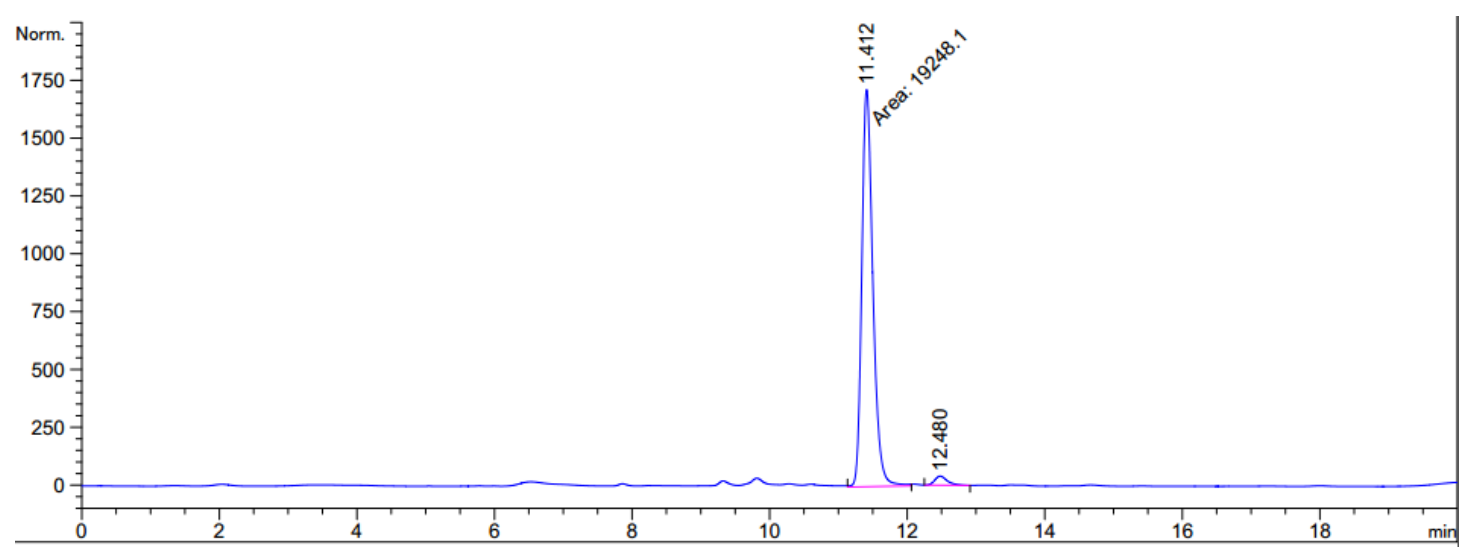

Signal 1: DAD1 B, Sig=254, 4 Ref=off

\begin{tabular}{|c|c|c|c|c|c|c|}
\hline $\begin{array}{c}\text { Peak } \\
\#\end{array}$ & $\begin{array}{c}\text { RetTime } \\
\text { [min] }\end{array}$ & Type & $\begin{array}{l}\text { Width } \\
\text { [min] }\end{array}$ & $\begin{array}{c}\text { Area } \\
{\left[\mathrm{mAU}^{\star} \mathrm{S}\right]}\end{array}$ & $\begin{array}{l}\text { Height } \\
\text { [mAU] }\end{array}$ & $\begin{array}{c}\text { Area } \\
\frac{8}{6}\end{array}$ \\
\hline 1 & 1141 & MM & 0.18 & 19248104 & 17169 & 710 \\
\hline 2 & 12.480 & BB & 0.1662 & 499.41776 & 39.43636 & 5290 \\
\hline
\end{tabular}


<smiles>CC(C)C(O)/C=C/C=C/c1ccccc1</smiles>

racemate for $\mathbf{2 c}$

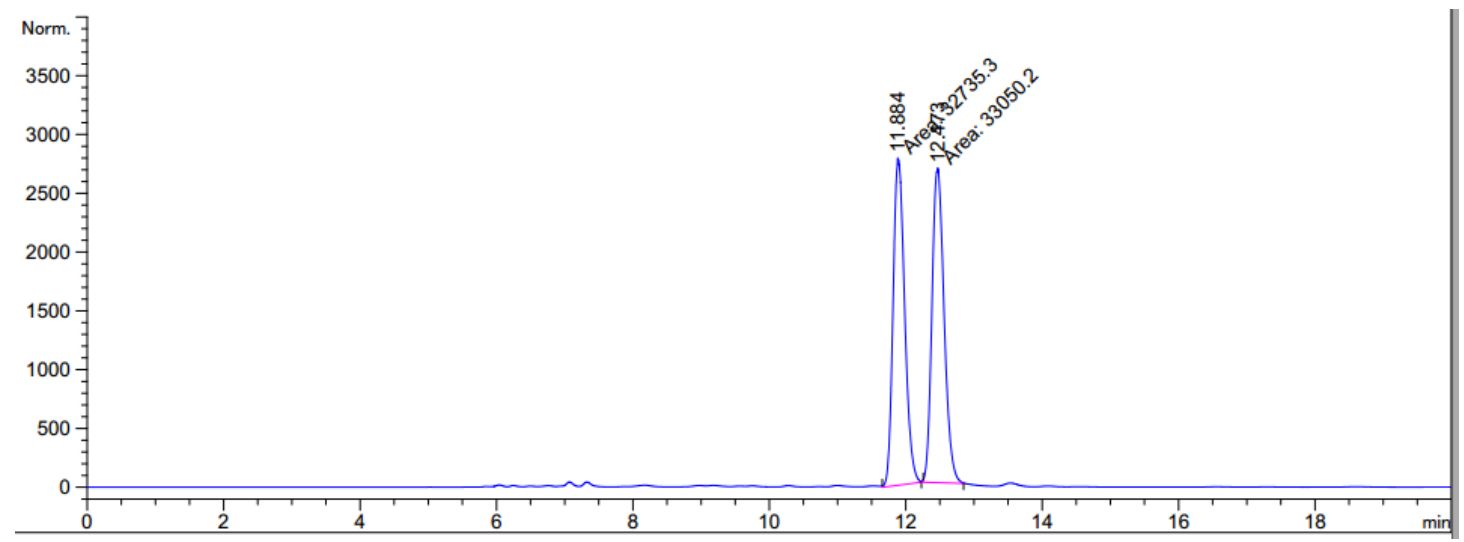

Signal 1: DAD1 E, Sig=280, 4 Ref=off

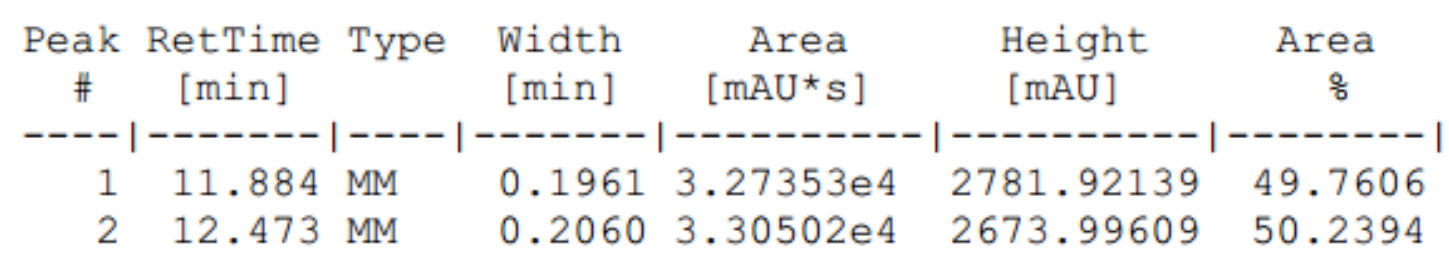<smiles>CC(C)C(O)/C=C/C=C/c1ccccc1</smiles>

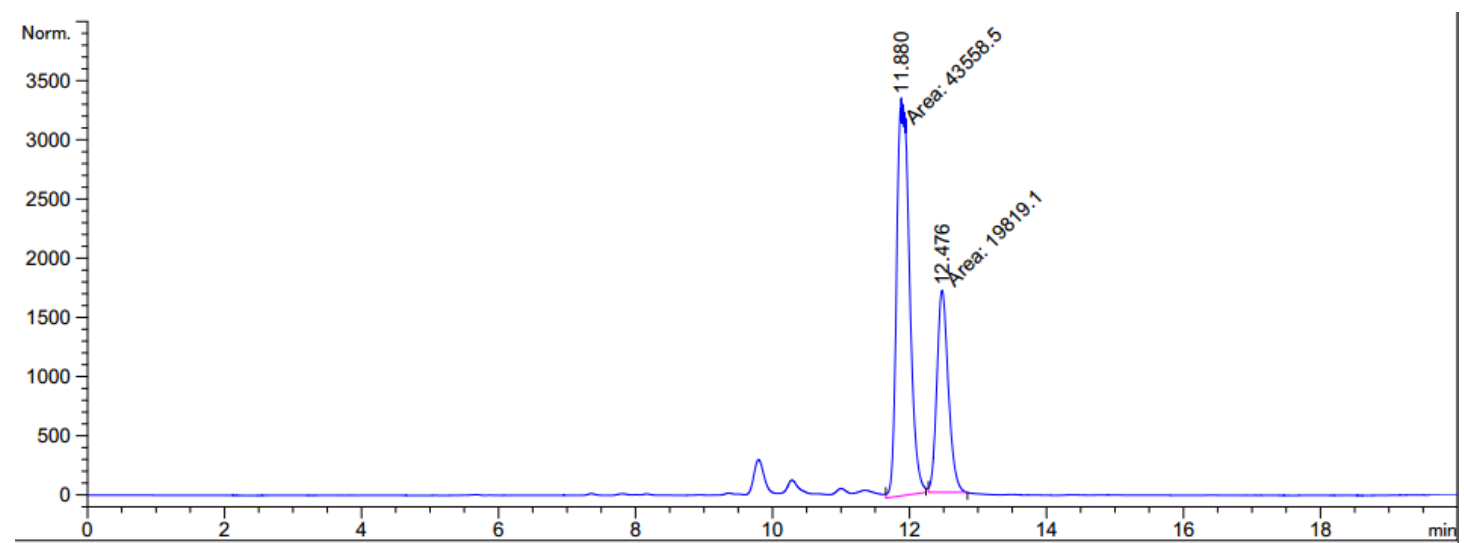

Signal 1: DAD1 E, Sig=280, 4 Ref=off

\begin{tabular}{|c|c|c|c|c|c|c|}
\hline $\begin{array}{c}\text { Peak } \\
\#\end{array}$ & $\begin{array}{c}\text { RetTime } \\
\text { [min] }\end{array}$ & Type & $\begin{array}{l}\text { Width } \\
\text { [min] }\end{array}$ & $\begin{array}{c}\text { Area } \\
{\left[\mathrm{mAU}^{*} \mathrm{~s}\right]}\end{array}$ & $\begin{array}{l}\text { Height } \\
{[\mathrm{mAU}]}\end{array}$ & $\begin{array}{c}\text { Area } \\
\frac{8}{\sigma}\end{array}$ \\
\hline-1 & & & & --- & & --1 \\
\hline 1 & 11.880 & MM & 0.2165 & $4.35585 e 4$ & 3353.92285 & 68.7286 \\
\hline 2 & 12.476 & MM & 0.1935 & $1.98191 \mathrm{e} 4$ & 1706.93652 & 31.2714 \\
\hline
\end{tabular}


<smiles>OC1CCC1=CC=Cc1ccccc1</smiles>

racemate for $\mathbf{2 d}$

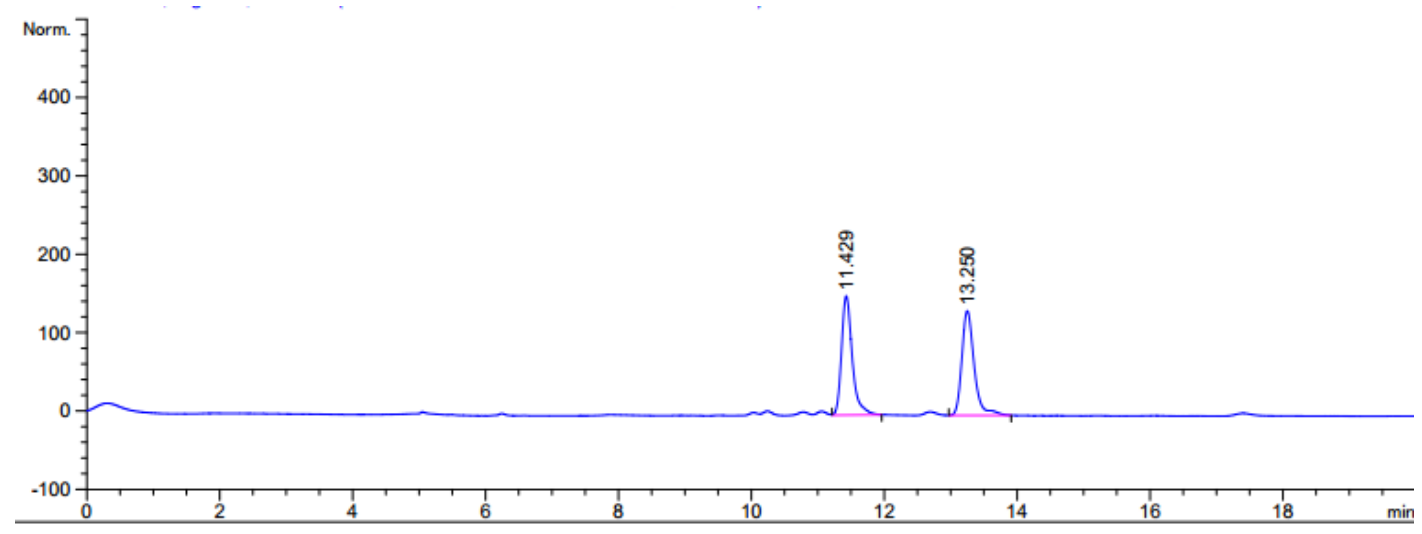

Signal 1: DAD1 B, Sig=254,4 Ref=off

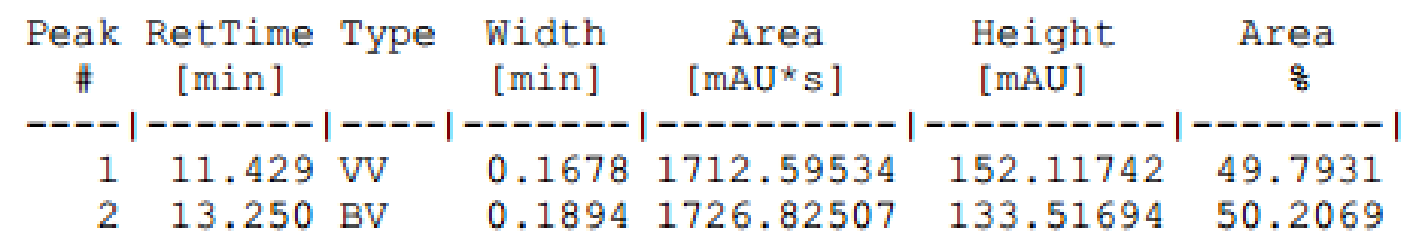<smiles>OC1CCC1=CC=Cc1ccccc1</smiles>

2d

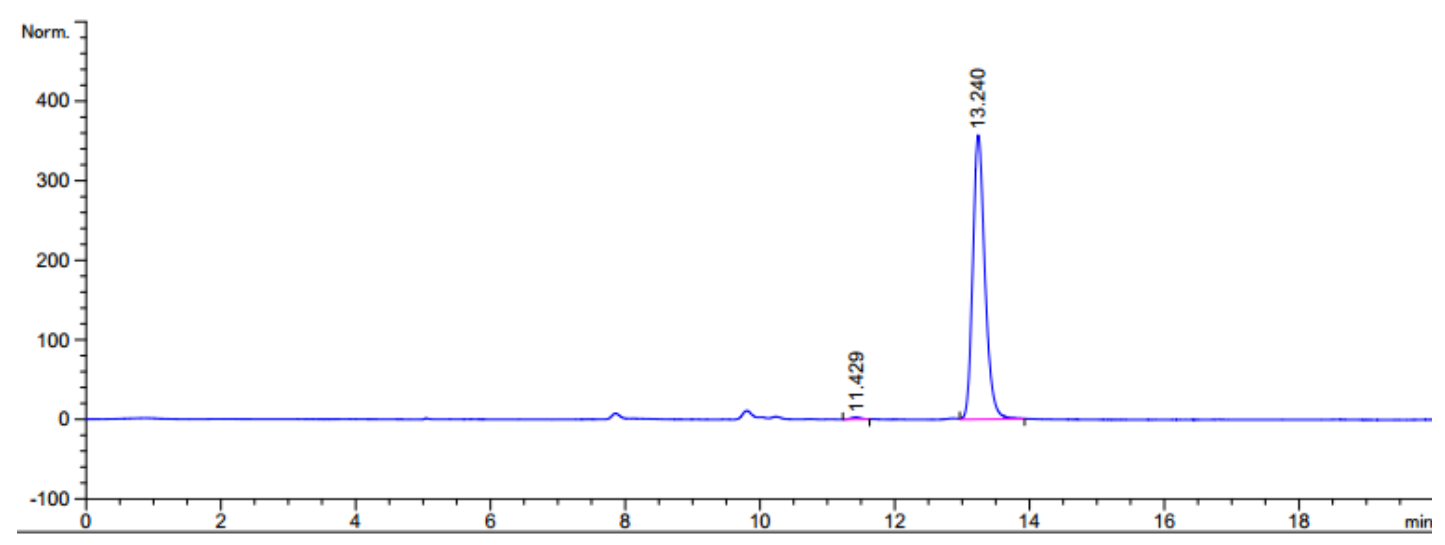

Signal 1: DAD1 B, Sig=254,4 Ref=off

\begin{tabular}{|c|c|c|c|c|c|c|}
\hline $\begin{array}{c}\text { Peak } \\
\#\end{array}$ & $\begin{array}{c}\text { RetTime } \\
\text { [min] }\end{array}$ & Type & $\begin{array}{c}\text { Width } \\
\text { [min] }\end{array}$ & $\begin{array}{c}\text { Area } \\
{\left[\mathrm{mAU}^{\star} \mathrm{s}\right]}\end{array}$ & $\begin{array}{l}\text { Height } \\
\text { [mAU] }\end{array}$ & $\begin{array}{c}\text { Area } \\
\text { 옿 }\end{array}$ \\
\hline$=-$ & -- & & & 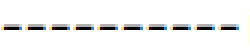 & -- & -1 \\
\hline 1 & 11.429 & BB & 0.1196 & 26.15461 & 2.60640 & 0.5814 \\
\hline 2 & 13.240 & VV & 0.1905 & 4472.66162 & 357.45590 & 99.4186 \\
\hline
\end{tabular}


<smiles>CC(/C=C/C(C)O)=C\c1ccccc1</smiles>

racemate for $\mathbf{2 e}$

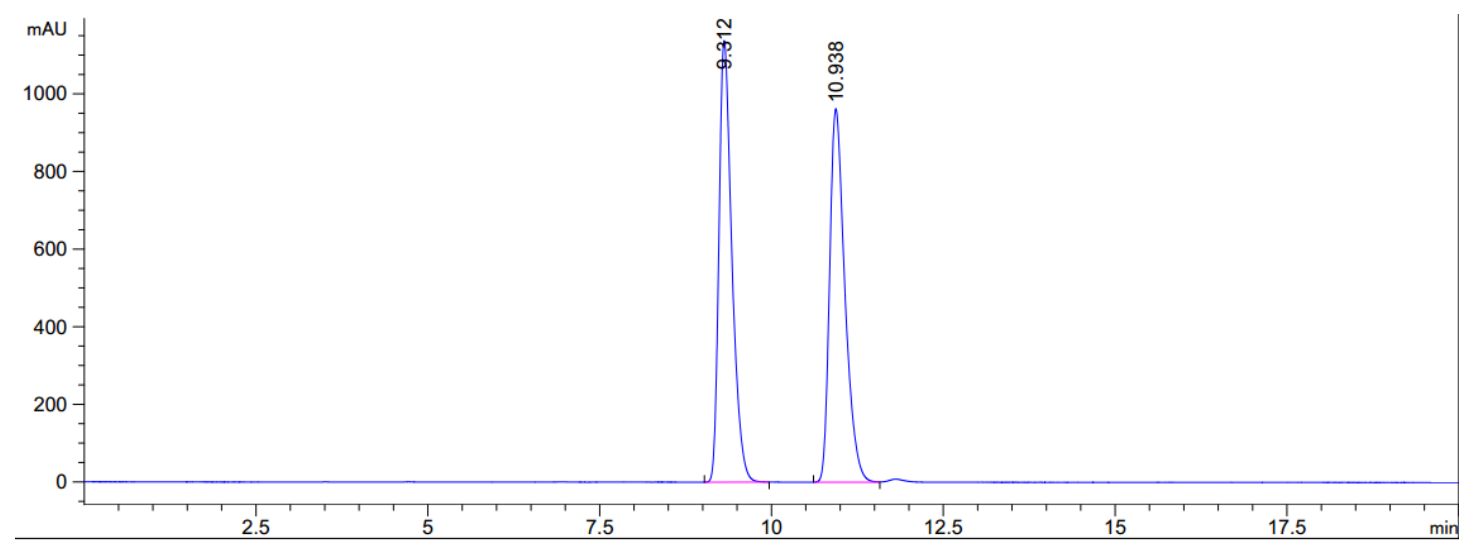

Signal 1: DAD1 E, Sig=280,4 Ref=off

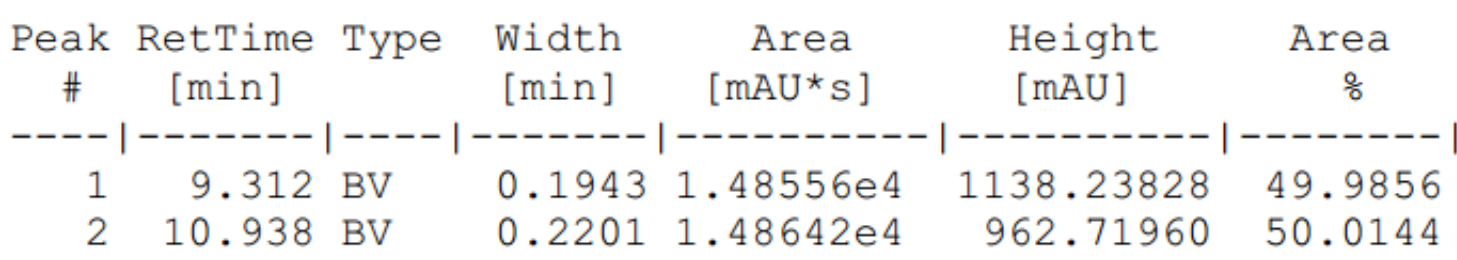<smiles>CC(/C=C/C(C)O)=C\c1ccccc1</smiles>

$2 e$

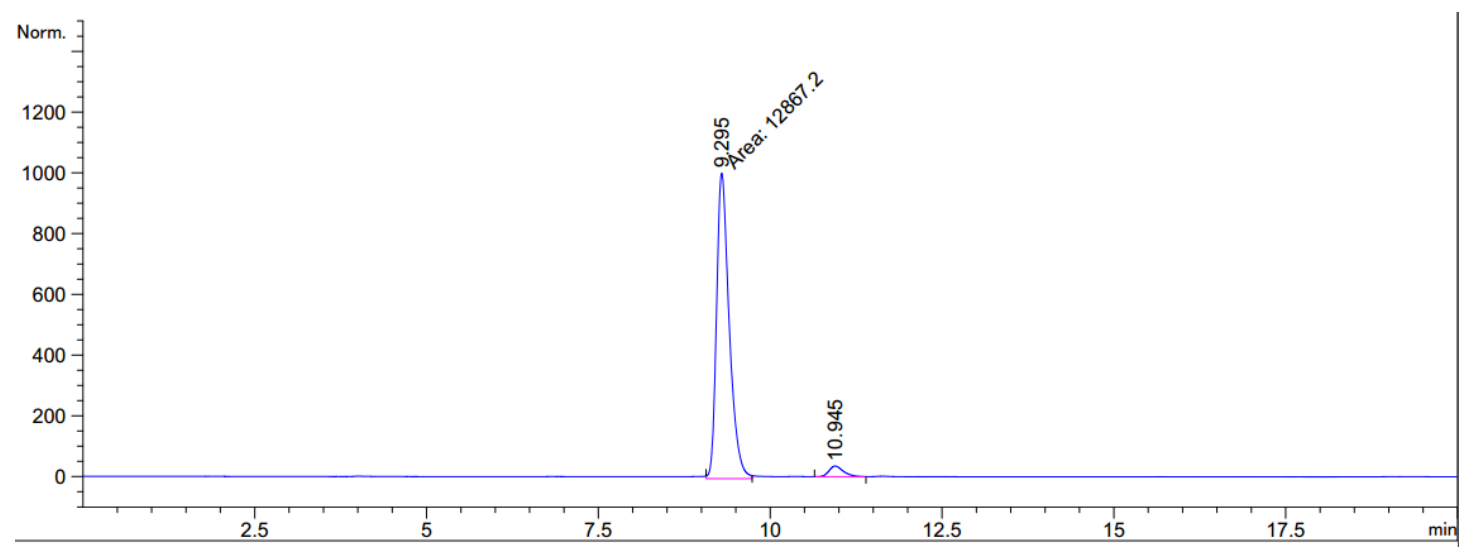

Signal 1: DAD1 E, Sig=280, 4 Ref=off

\begin{tabular}{|c|c|c|c|c|c|c|}
\hline $\begin{array}{c}\text { Peak } \\
\#\end{array}$ & $\begin{array}{c}\text { RetTime } \\
\text { [min] }\end{array}$ & Type & $\begin{array}{l}\text { Width } \\
\text { [min] }\end{array}$ & $\begin{array}{c}\text { Area } \\
{\left[\mathrm{mAU}^{\star} \mathrm{s}\right]}\end{array}$ & $\begin{array}{l}\text { Height } \\
{[\mathrm{mAU}]}\end{array}$ & $\begin{array}{c}\text { Area } \\
\frac{\%}{\delta}\end{array}$ \\
\hline & & & ------- & ---------- & & \\
\hline 1 & 9.295 & MM & 0.2132 & $1.28672 \mathrm{e} 4$ & 1005.71558 & 96.1827 \\
\hline 2 & 10.945 & BV & 0.1867 & 510.68045 & 35.21732 & 3.8173 \\
\hline
\end{tabular}


<smiles>CC(O)C=CC(Br)=Cc1ccccc1</smiles>

racemate for $\mathbf{2 f}$

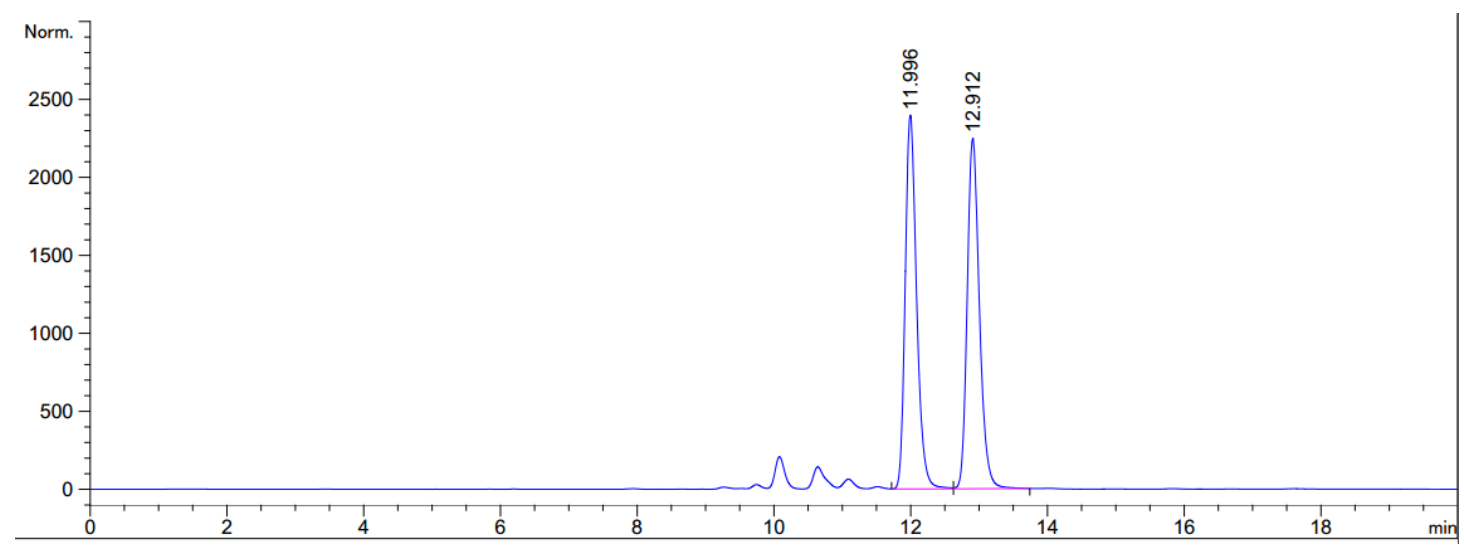

Signal 1: DAD1 E, Sig=280,4 Ref=off

\begin{tabular}{|c|c|c|c|c|c|c|}
\hline $\begin{array}{c}\text { Peak } \\
\#\end{array}$ & $\begin{array}{c}\text { RetTime } \\
\text { [min] }\end{array}$ & Type & $\begin{array}{l}\text { Width } \\
\text { [min] }\end{array}$ & $\begin{array}{c}\text { Area } \\
{\left[\mathrm{mAU}^{*} \mathrm{~S}\right]}\end{array}$ & $\begin{array}{l}\text { Height } \\
\text { [mAU] }\end{array}$ & $\begin{array}{c}\text { Area } \\
\%\end{array}$ \\
\hline-- & & & & --- & 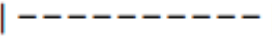 & --1 \\
\hline 1 & 11.9 & BV & 0.13 & $2.77347 e 4$ & 74854 & 148 \\
\hline 2 & 12.912 & VV & 0.1464 & $2.78294 \mathrm{e} 4$ & 2248.31738 & 0852 \\
\hline
\end{tabular}<smiles>CC(O)C=CC(Br)=Cc1ccccc1</smiles>

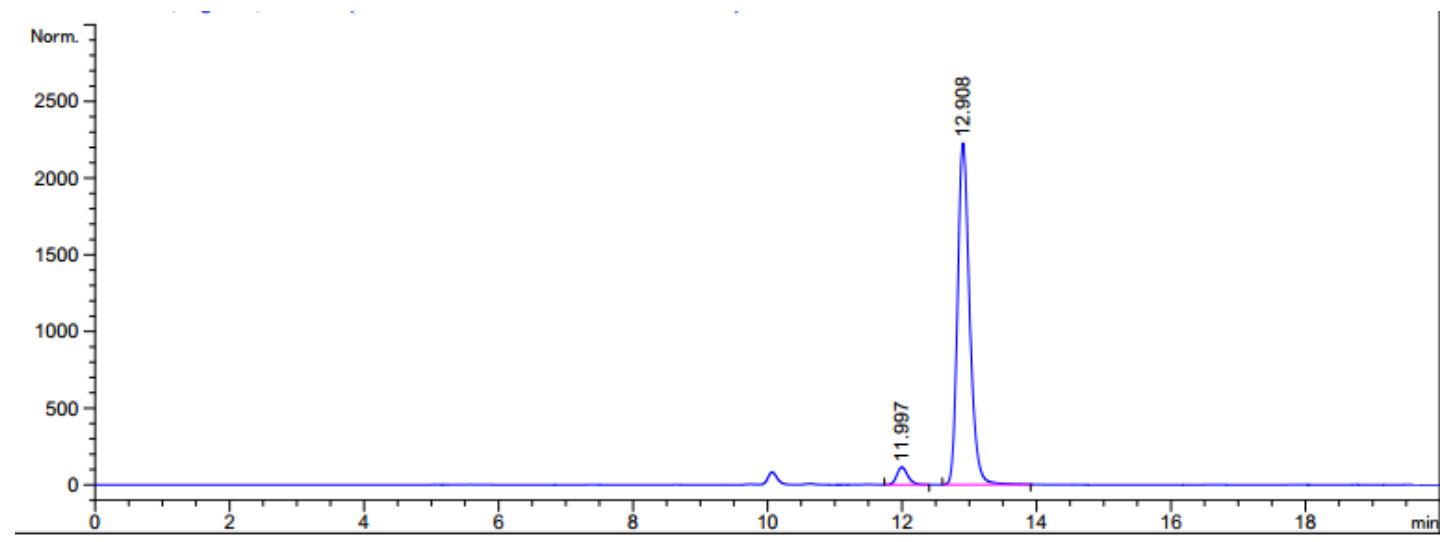

Signal 1: DAD1 E, Sig=280,4 Ref=off

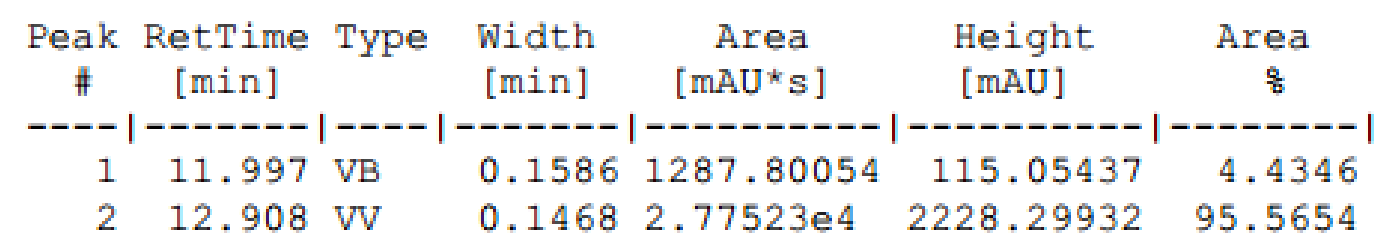


<smiles>CC(O)C=CC=Cc1cccc(C(F)(F)F)c1</smiles>

racemate for $\mathbf{2 g}$

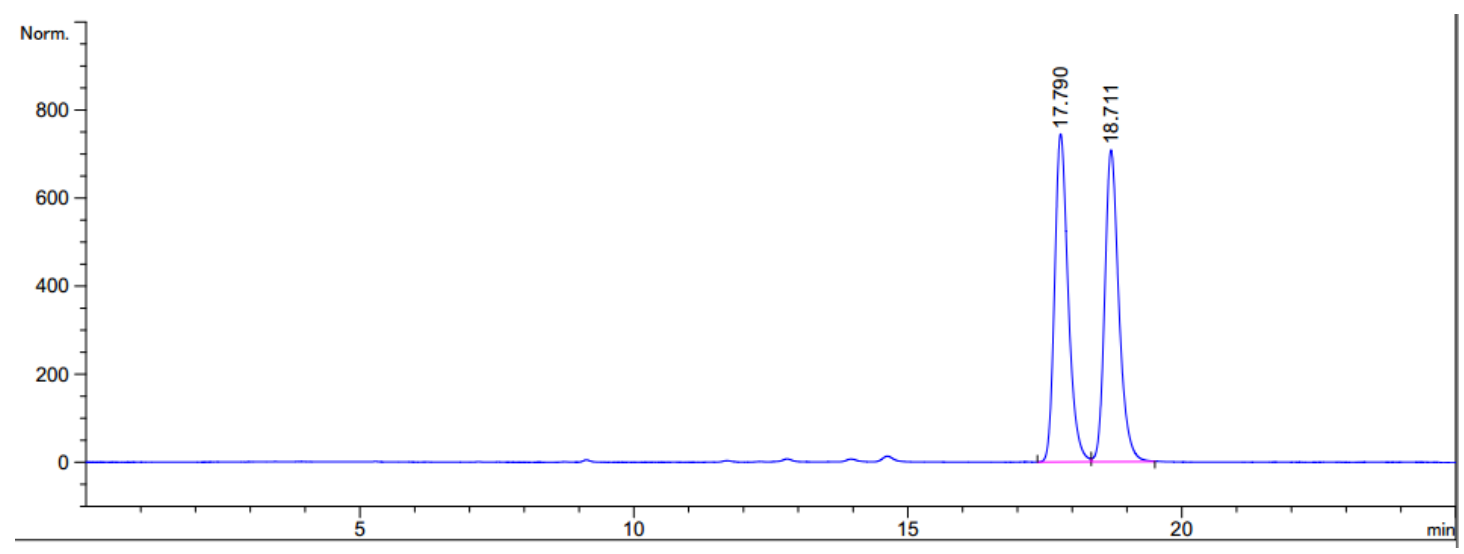

Signal 1: DAD1 E, Sig=280, 4 Ref=off

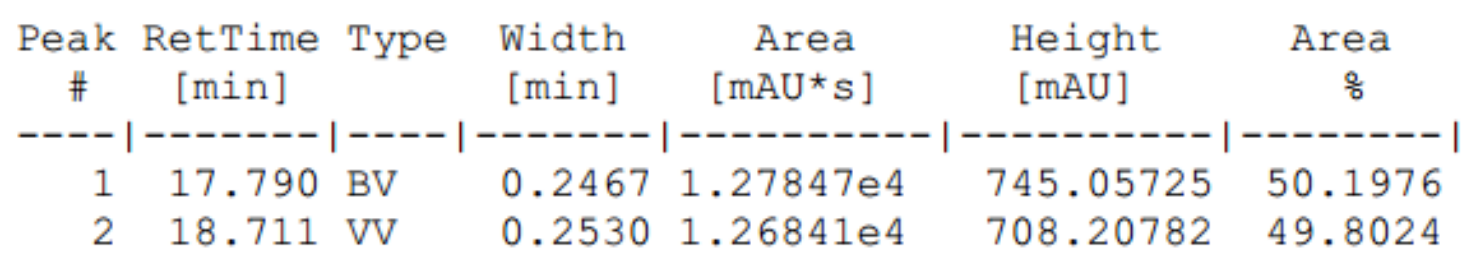<smiles>CC(O)C=CC=Cc1cccc(C(F)(F)F)c1</smiles>

$2 g$

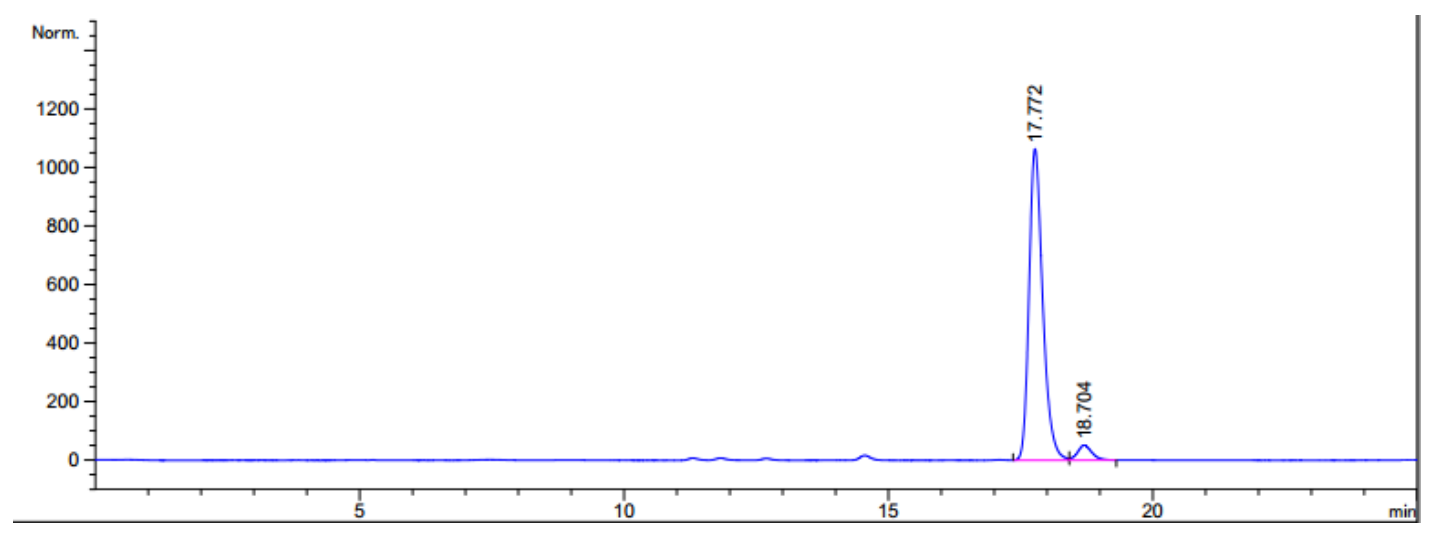

Signal 1: DAD1 E, Sig=280, 4 Ref $=$ off

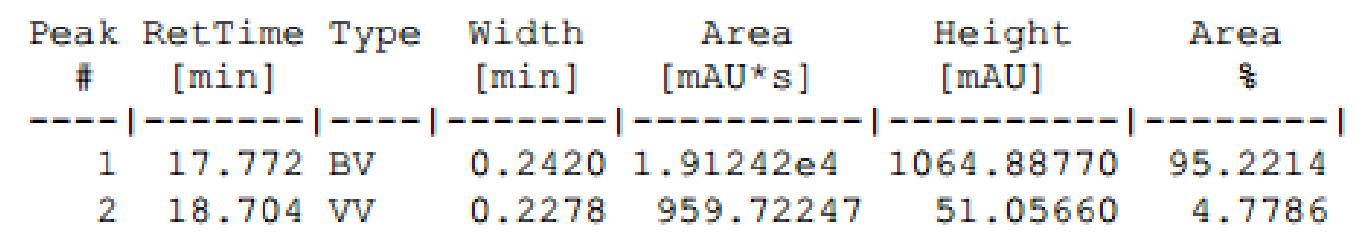


<smiles>Cc1ccccc1/C=C/C=C/C(C)O</smiles>

racemate for $\mathbf{2 h}$

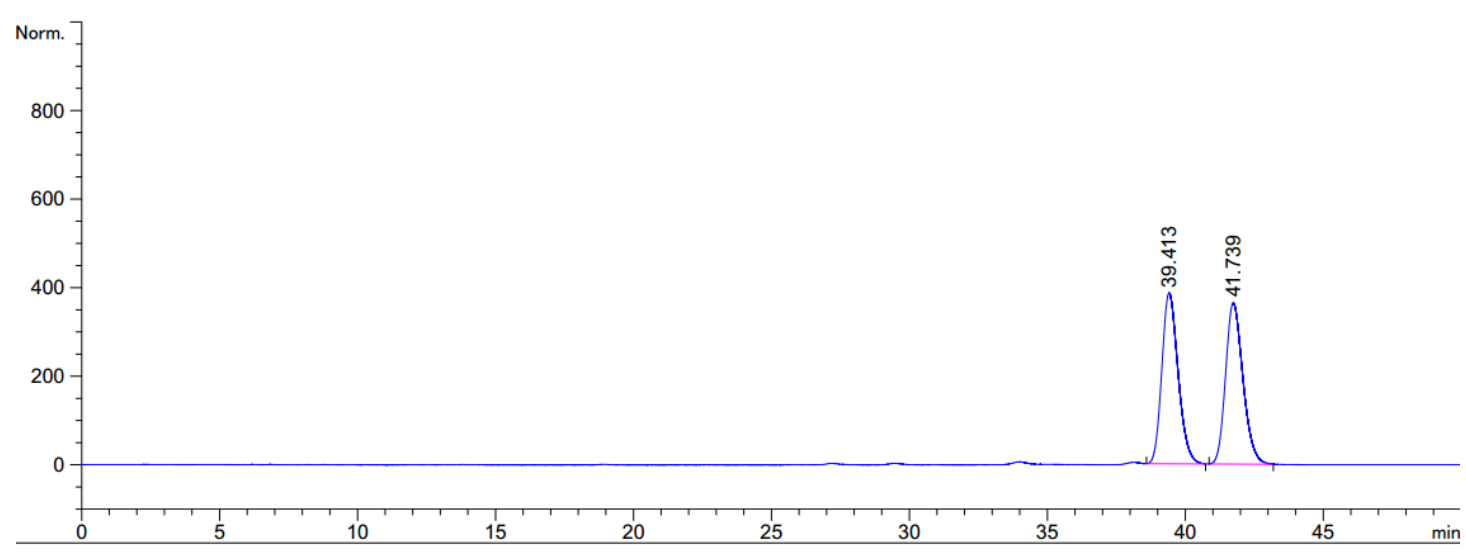

Signal 1: DAD1 E, Sig=280,4 Ref=off

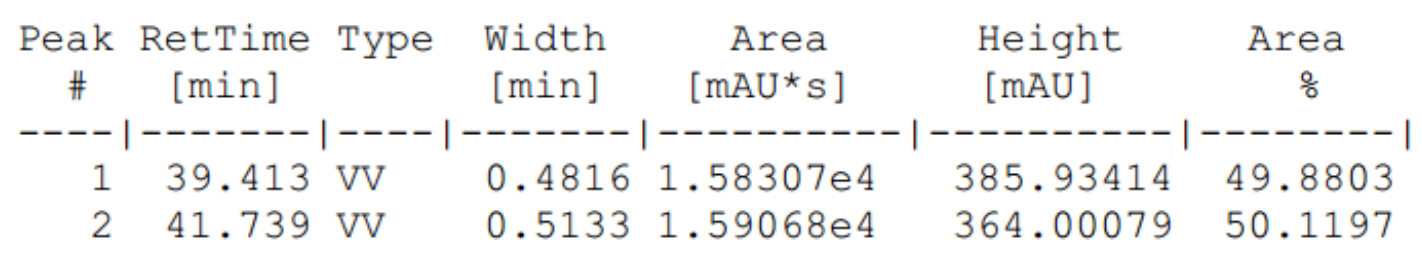<smiles>Cc1ccccc1/C=C/C=C/C(C)O</smiles>

$2 \mathrm{~h}$

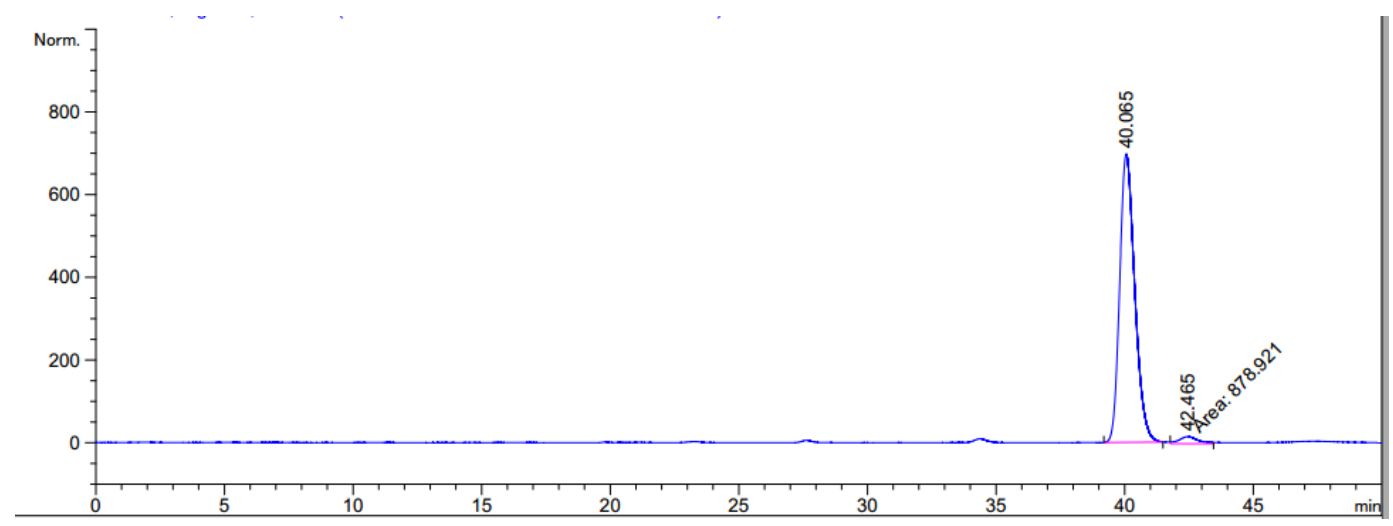

Signal 1: DAD1 E, Sig=280,4 Ref=off

\begin{tabular}{|c|c|c|c|c|c|c|}
\hline $\begin{array}{c}\text { Peak } \\
\#\end{array}$ & $\begin{array}{l}\text { RetTime } \\
\text { [min] }\end{array}$ & Type & $\begin{array}{l}\text { Width } \\
\text { [min] }\end{array}$ & $\begin{array}{c}\text { Area } \\
{\left[\mathrm{mAU}^{\star} \mathrm{s}\right]}\end{array}$ & $\begin{array}{l}\text { Height } \\
\text { [mAU] }\end{array}$ & $\begin{array}{c}\text { Area } \\
\frac{8}{6}\end{array}$ \\
\hline- & $\mid------$ & & & --------- & ------- & $-------\mid$ \\
\hline 1 & 40.065 & VV & 0.4768 & $2.83649 \mathrm{e} 4$ & 696.97681 & 96.9945 \\
\hline 2 & 42.465 & MM & 0.8596 & 878.92078 & 17.04056 & 3.0055 \\
\hline
\end{tabular}


<smiles>CC(O)/C=C/C=C/c1ccc(Cl)cc1</smiles>

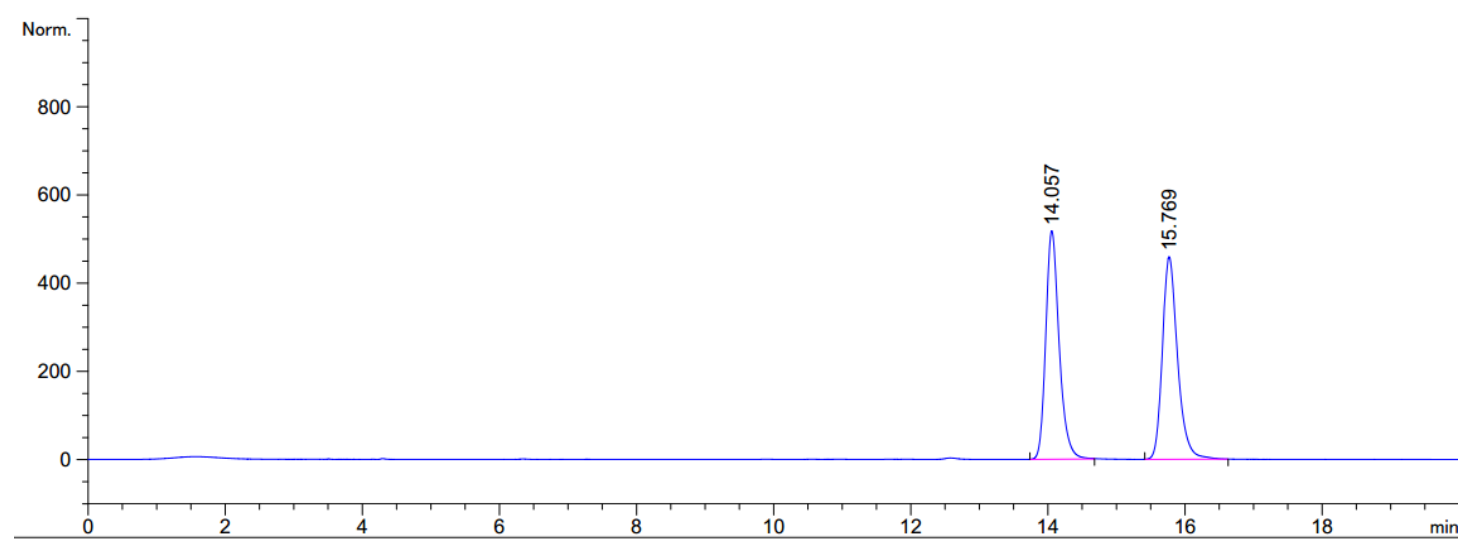

Signal 1: DAD1 E, Sig=280, 4 Ref=off

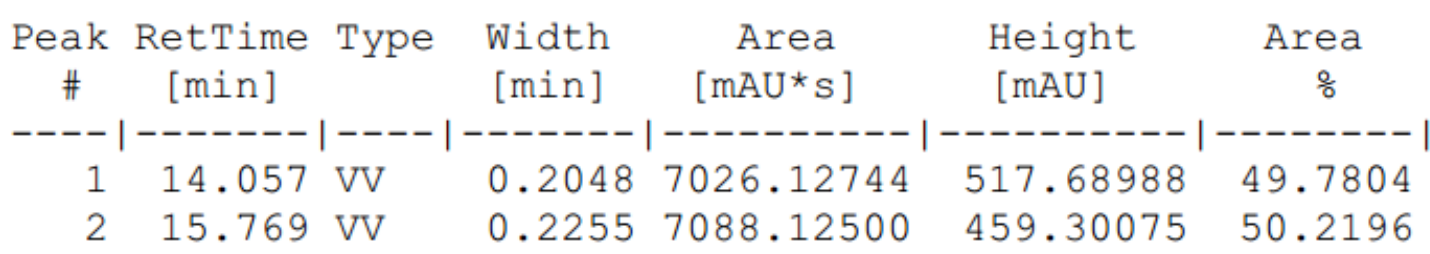<smiles>C[C@H](O)/C=C/C=C/c1ccc(Cl)cc1</smiles>

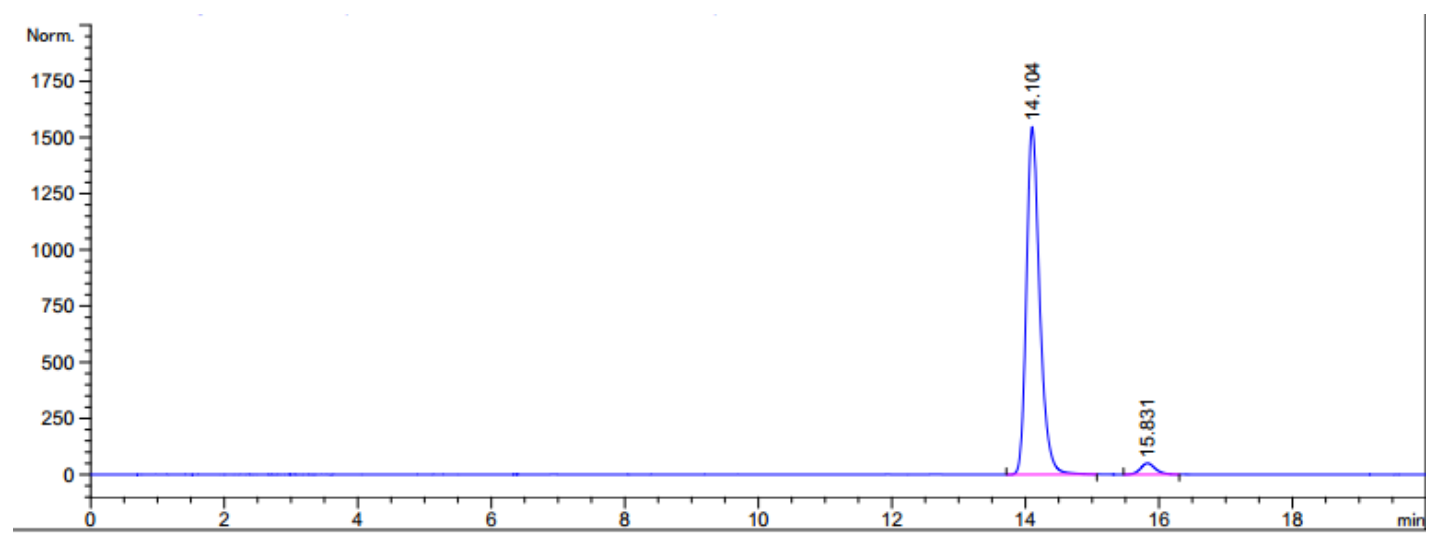

Signal 1: DAD1 E, Sig=280,4 Ref=off

\begin{tabular}{|c|c|c|c|c|c|c|}
\hline $\begin{array}{c}\text { Peak } \\
\#\end{array}$ & $\begin{array}{c}\text { RetTime } \\
\text { [min] }\end{array}$ & Type & $\begin{array}{l}\text { Width } \\
\text { [min] }\end{array}$ & $\begin{array}{c}\text { Area } \\
{\left[\mathrm{mAU}^{\star} \mathrm{s}\right]}\end{array}$ & $\begin{array}{l}\text { Height } \\
\text { [mAU] }\end{array}$ & $\begin{array}{c}\text { Area } \\
\text { 옹 }\end{array}$ \\
\hline 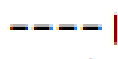 & & & & --- & & $-2=-1$ \\
\hline $\begin{array}{l}1 \\
2\end{array}$ & $\begin{array}{l}14.104 \\
15.831\end{array}$ & $\begin{array}{l}\text { BV } \\
\text { BV }\end{array}$ & $\begin{array}{l}0.1937 \\
0.1889\end{array}$ & $\begin{array}{c}2.13838 \mathrm{e} 4 \\
778.56598\end{array}$ & $\begin{array}{r}1547.52356 \\
50.20196\end{array}$ & $\begin{array}{r}96.4870 \\
3.5130\end{array}$ \\
\hline
\end{tabular}


<smiles>CC(O)/C=C/C=C/c1ccc(Br)cc1</smiles>

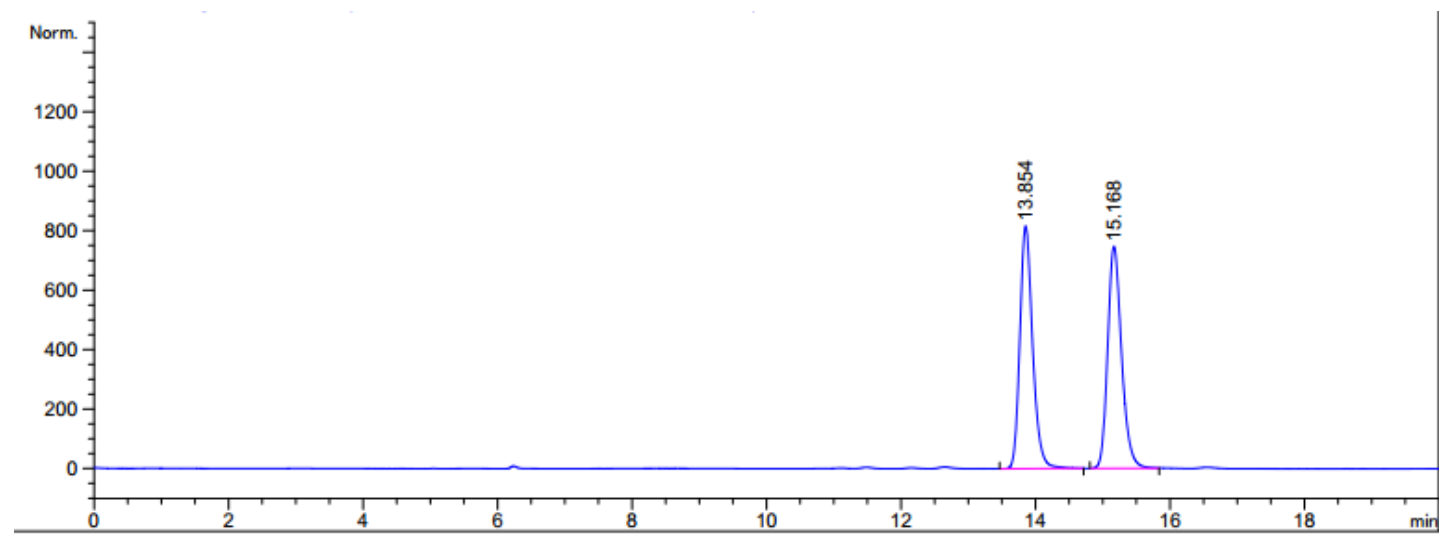

Signal 1: DAD1 E, Sig=280, 4 Ref=off

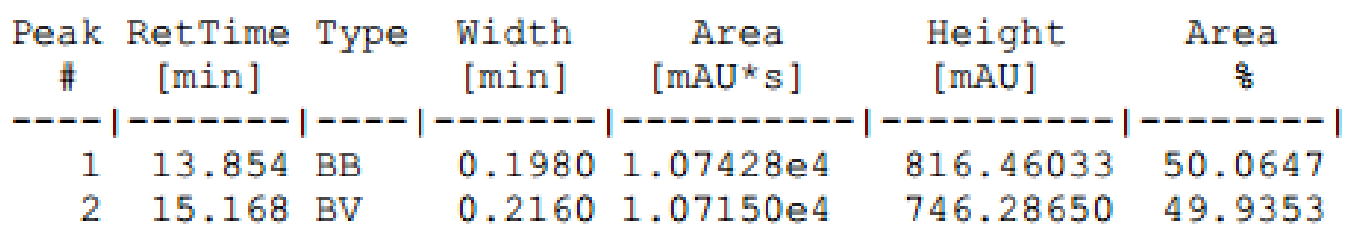<smiles>C[C@H](O)/C=C/C=C/c1ccc(Br)cc1</smiles>

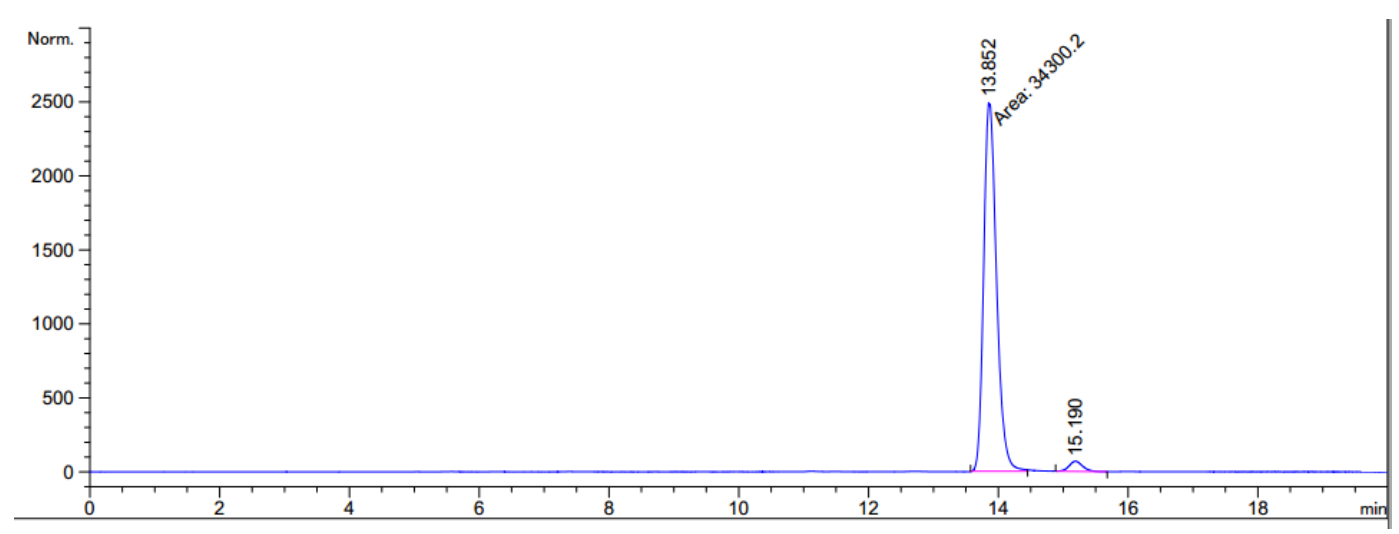

Signal 1: DAD1 E, Sig=280,4 Ref=off

\begin{tabular}{|c|c|c|c|c|c|c|}
\hline $\begin{array}{c}\text { Peak } \\
\#\end{array}$ & $\begin{array}{c}\text { RetTime } \\
\text { [min] }\end{array}$ & Type & $\begin{array}{l}\text { Width } \\
\text { [min] }\end{array}$ & $\begin{array}{c}\text { Area } \\
{\left[\mathrm{mAU}^{*} \mathrm{~s}\right]}\end{array}$ & $\begin{array}{l}\text { Height } \\
\text { [mAU] }\end{array}$ & $\begin{array}{c}\text { Area } \\
\frac{8}{6}\end{array}$ \\
\hline---- & & & ------- & ---- & |----------| & -------1 \\
\hline 1 & 13.852 & MM & 0.2293 & $3.43002 \mathrm{e} 4$ & 2493.22095 & 97.2078 \\
\hline 2 & 15.190 & VB & 0.1840 & 985.23712 & 68.60590 & 2.7922 \\
\hline
\end{tabular}


<smiles>CC(O)C=CC=Cc1ccc(F)cc1</smiles>

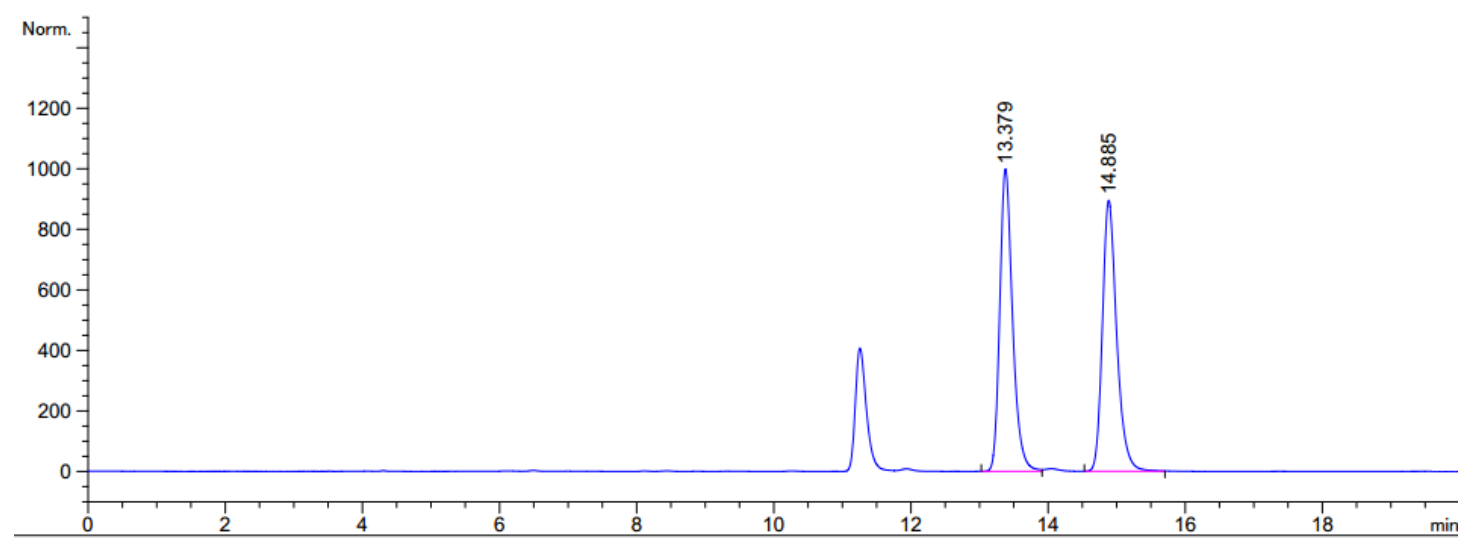

Signal 1: DAD1 E, Sig=280, 4 Ref=off

\begin{tabular}{|c|c|c|c|c|c|c|}
\hline $\begin{array}{c}\text { Peak } \\
\#\end{array}$ & $\begin{array}{c}\text { RetTime } \\
\text { [min] }\end{array}$ & Type & $\begin{array}{l}\text { Width } \\
\text { [min] }\end{array}$ & $\begin{array}{c}\text { Area } \\
{\left[\mathrm{mAU}^{*} \mathrm{~s}\right]}\end{array}$ & $\begin{array}{l}\text { Height } \\
{[\mathrm{mAU}]}\end{array}$ & $\begin{array}{c}\text { Area } \\
\quad \%\end{array}$ \\
\hline- & & & & ---------- & 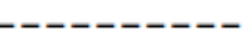 & \\
\hline 1 & 13.379 & VV & 0.1929 & 1. $29328 e 4$ & 999.75269 & 513 \\
\hline 2 & 14.885 & BV & 0.2168 & $1.29580 \mathrm{e} 4$ & 895.49182 & 50.0487 \\
\hline
\end{tabular}<smiles>C[C@H](O)/C=C/C=C/c1ccc(F)cc1</smiles>

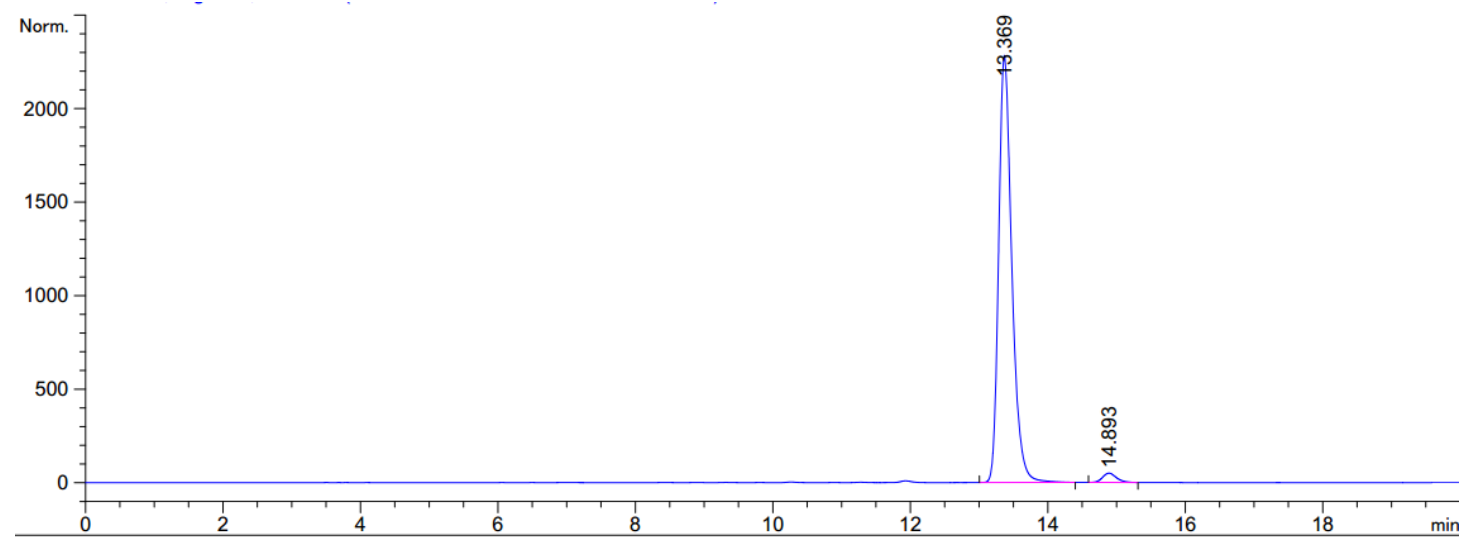

Signal 1: DAD1 E, Sig=280,4 Ref=off

\begin{tabular}{|c|c|c|c|c|c|c|}
\hline $\begin{array}{c}\text { Peak } \\
\#\end{array}$ & $\begin{array}{l}\text { RetTime } \\
\text { [min] }\end{array}$ & Type & $\begin{array}{l}\text { Width } \\
\text { [min] }\end{array}$ & $\begin{array}{c}\text { Area } \\
{\left[\mathrm{mAU}{ }^{\star} \mathrm{S}\right]}\end{array}$ & $\begin{array}{l}\text { Height } \\
{[\mathrm{mAU}]}\end{array}$ & $\begin{array}{c}\text { Area } \\
\frac{\%}{\delta}\end{array}$ \\
\hline$=--$ & $-0-0-0$ & & ------ & ----------- & |---------- & --- \\
\hline 1 & 13.369 & VV & 0.158 & $3.03403 e 4$ & 2283.40894 & 97.7401 \\
\hline 2 & 14.893 & VV & 0.172 & 701.52496 & 49.87486 & 2.2599 \\
\hline
\end{tabular}


<smiles>COc1ccc(/C=C/C=C/C(C)O)cc1</smiles>

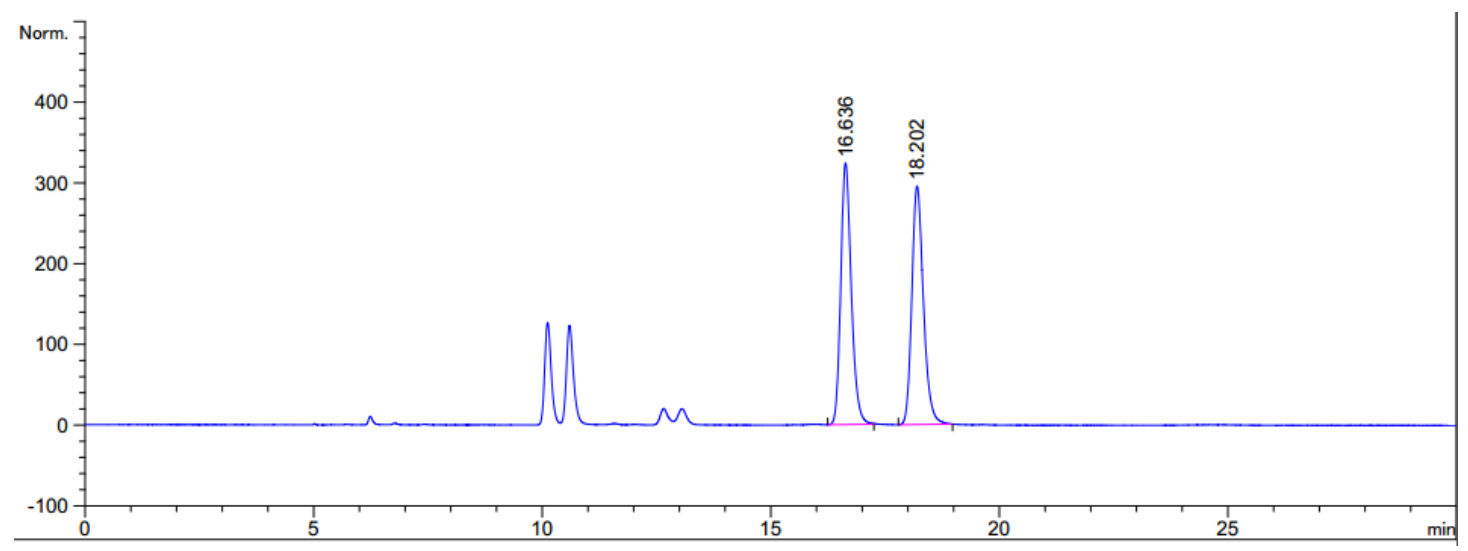

Signal 1: DAD1 E, Sig=280, 4 Ref=off

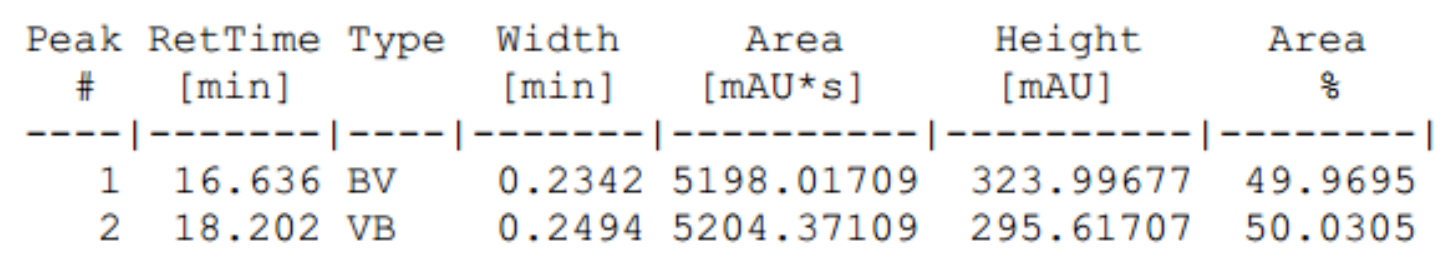<smiles>COc1ccc(/C=C/C=C/C(C)O)cc1</smiles>

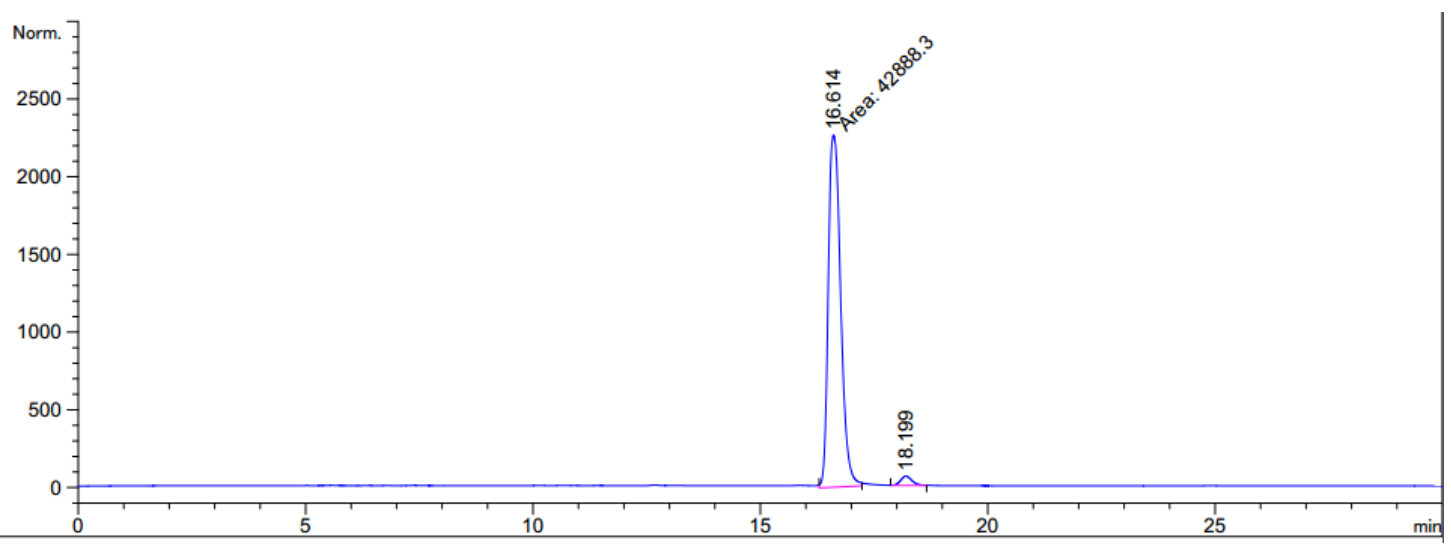

Signal 1: DAD1 E, Sig=280,4 Ref=off

\begin{tabular}{|c|c|c|c|c|c|c|}
\hline $\begin{array}{c}\text { Peak } \\
\#\end{array}$ & $\begin{array}{c}\text { RetTime } \\
\text { [min] }\end{array}$ & Type & $\begin{array}{c}\text { Width } \\
\text { [min] }\end{array}$ & $\begin{array}{c}\text { Area } \\
{\left[\mathrm{mAU}^{\star} \mathrm{s}\right]}\end{array}$ & $\begin{array}{l}\text { Height } \\
\text { [mAU] }\end{array}$ & $\begin{array}{c}\text { Area } \\
\frac{8}{\sigma}\end{array}$ \\
\hline$=-$ & & & ------ & --------- & |-------- & ------- \\
\hline 1 & 16.61 & MM & 0.3156 & $4.28883 e 4$ & 2265.06372 & 97.6415 \\
\hline 2 & 18.199 & VV & 0.2053 & 1035.96643 & 59.87758 & 2.3585 \\
\hline
\end{tabular}


<smiles>CC(O)/C=C/C=C/c1ccc(N(C)C)cc1</smiles>

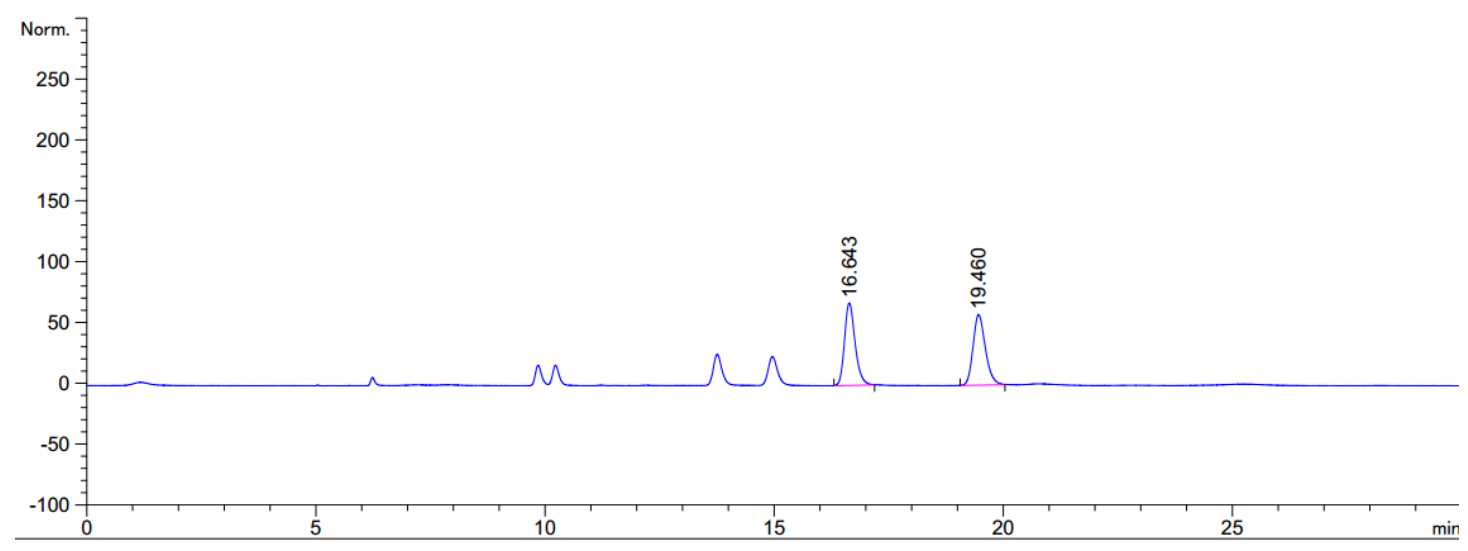

Signal 1: DAD1 H, Sig=290,4 Ref=off

\begin{tabular}{|c|c|c|c|c|c|c|}
\hline eak & $\begin{array}{l}\text { RetTime } \\
\text { [min] }\end{array}$ & Type & $\begin{array}{l}\text { Width } \\
\text { [min] }\end{array}$ & $\begin{array}{c}\text { Area } \\
{\left[\mathrm{mAU}^{*} \mathrm{~s}\right]}\end{array}$ & $\begin{array}{l}\text { Height } \\
\text { [MAU] }\end{array}$ & $\begin{array}{c}\text { Area } \\
\frac{\circ}{\delta}\end{array}$ \\
\hline 1 & & & & 8 & & \\
\hline & 60 & & 0 & 1086.1785 & 8.0 & 38 \\
\hline
\end{tabular}<smiles>CC(O)/C=C/C=C/c1ccc(N(C)C)cc1</smiles>

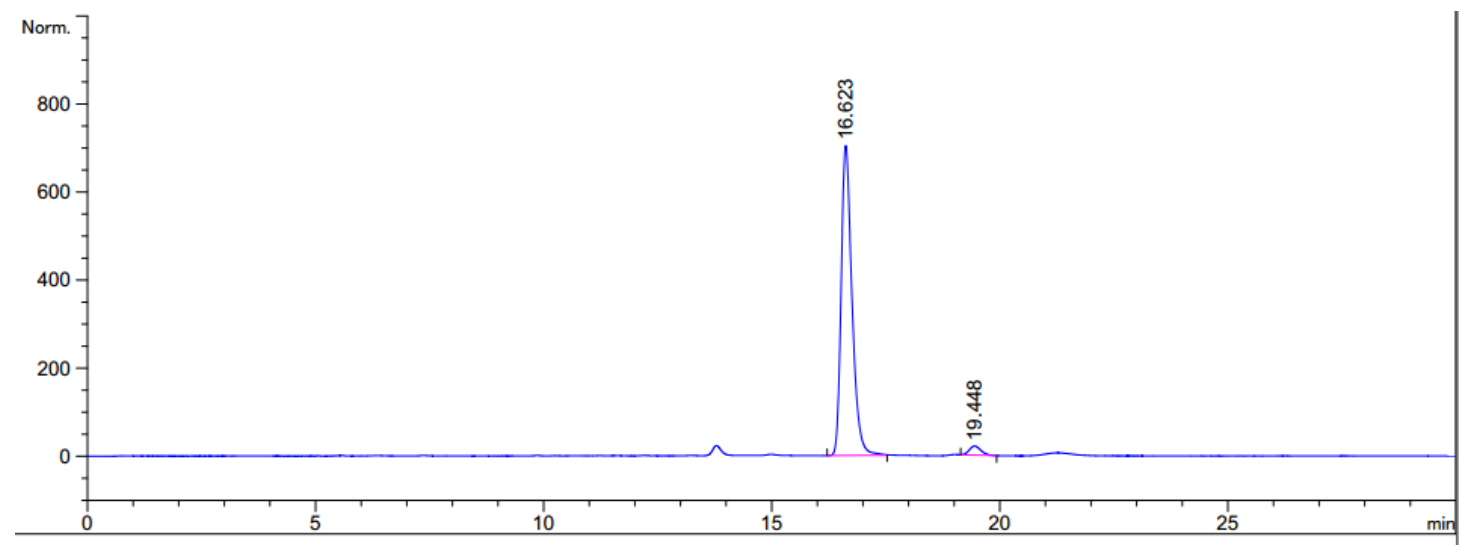

Signal 1: DAD1 H, Sig=290, 4 Ref=off

\begin{tabular}{|c|c|c|c|c|c|c|}
\hline $\begin{array}{c}\text { Peak } \\
\#\end{array}$ & $\begin{array}{c}\text { RetTime } \\
\text { [min] }\end{array}$ & Type & $\begin{array}{l}\text { Width } \\
\text { [min] }\end{array}$ & $\begin{array}{c}\text { Area } \\
{\left[\mathrm{mAU}^{\star} \mathrm{S}\right]}\end{array}$ & $\begin{array}{l}\text { Height } \\
\text { [mAU] }\end{array}$ & $\begin{array}{c}\text { Area } \\
\frac{8}{\sigma}\end{array}$ \\
\hline 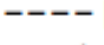 & & & & --------0 & ---------1 & ------ \\
\hline 1 & 16.623 & BV & 0.2325 & $1.16120 \mathrm{e} 4$ & 703.76849 & 96.9861 \\
\hline 2 & 19.448 & BB & 0.2068 & 360.85001 & 20.55704 & 3.0139 \\
\hline
\end{tabular}


<smiles>CC(O)/C=C/C=C/c1ccc([N+](=O)[O-])cc1</smiles>

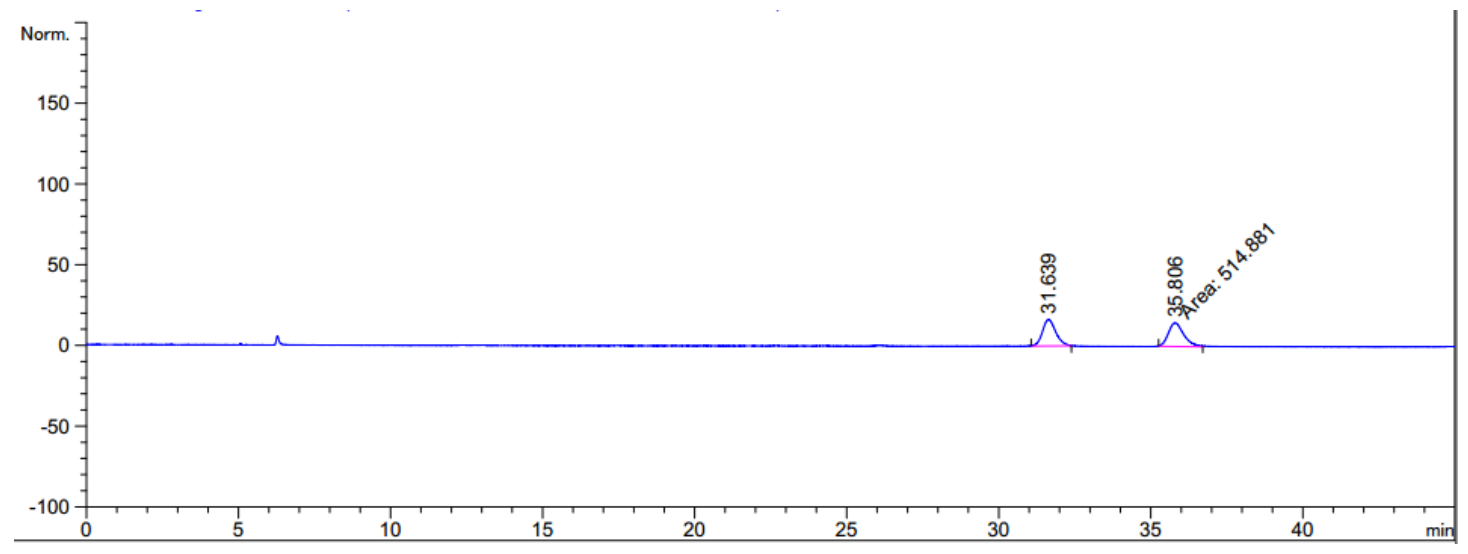

Signal 1: DAD1 B, Sig=254,4 Ref=off

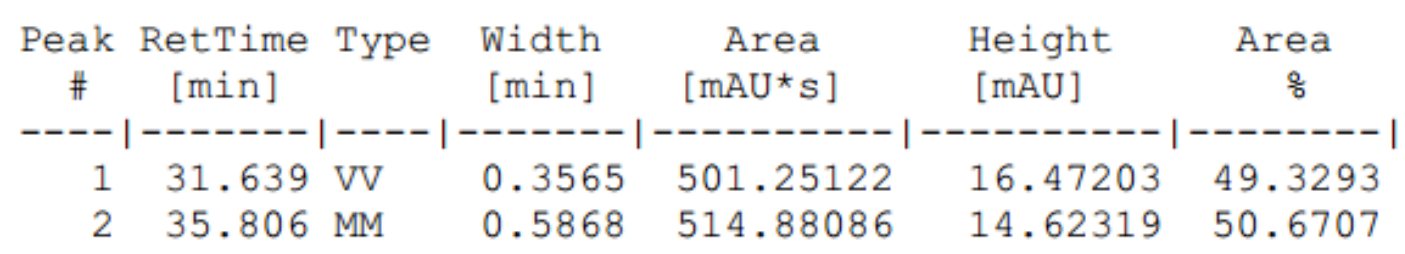<smiles>C[C@H](O)/C=C/C=C/c1ccc([N+](=O)[O-])cc1</smiles>

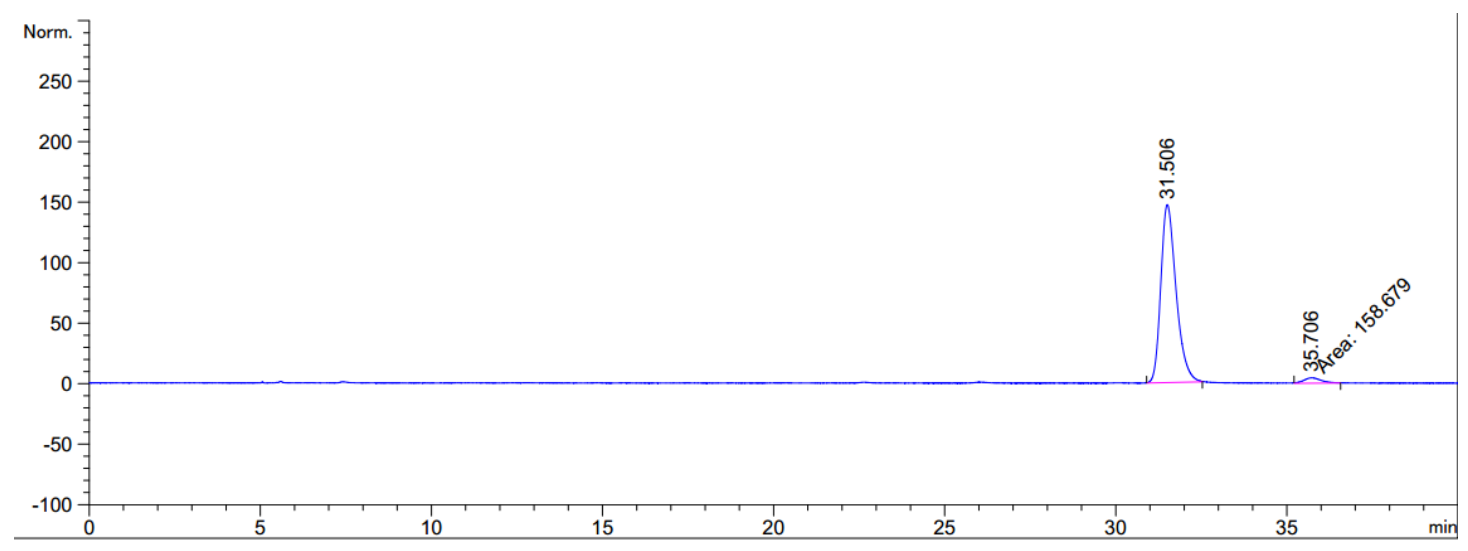

Signal 1: DAD1 B, Sig=254, 4 Ref=off

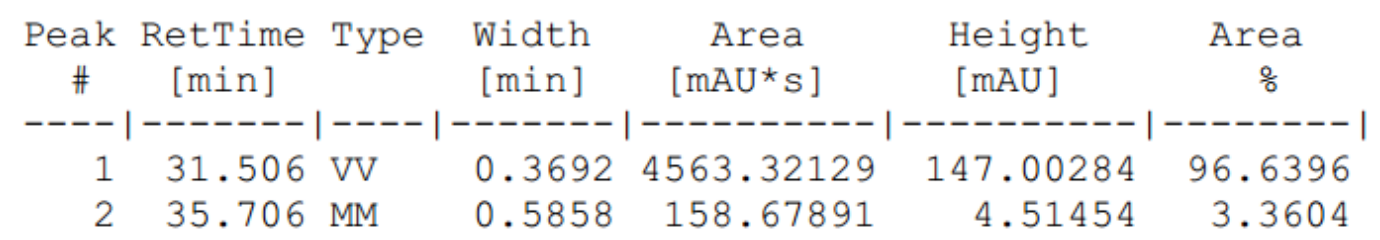



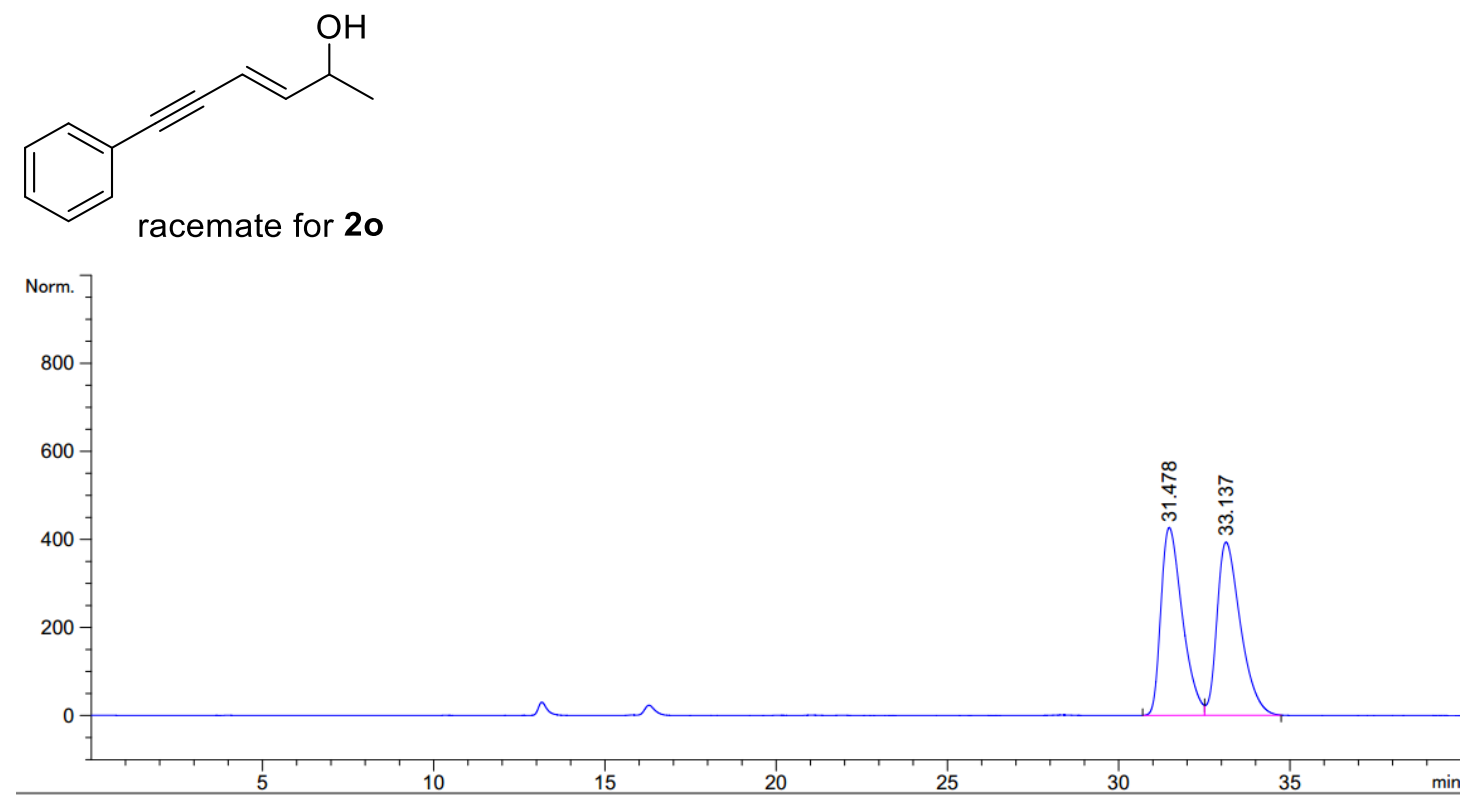

Signal 1: DAD1 E, Sig=280,4 Ref=off

\begin{tabular}{|c|c|c|c|c|c|c|}
\hline $\begin{array}{c}\text { Peak } \\
\#\end{array}$ & $\begin{array}{l}\text { RetTime } \\
\text { [min] }\end{array}$ & Type & $\begin{array}{c}\text { Width } \\
\text { [min] }\end{array}$ & $\begin{array}{c}\text { Area } \\
{\left[\mathrm{mAU}^{\star} \mathrm{S}\right]}\end{array}$ & $\begin{array}{l}\text { Height } \\
\text { [mAU] }\end{array}$ & $\begin{array}{c}\text { Area } \\
\frac{\circ}{0}\end{array}$ \\
\hline & & & & & & \\
\hline 1 & 31.478 & VV & 0.5 & $1.86236 \mathrm{e} 4$ & 6334 & 7022 \\
\hline 2 & 33.137 & VV & 0. & $1.88467 e 4$ & 1058 & 2978 \\
\hline
\end{tabular}<smiles>CC(O)/C=C/C#Cc1ccccc1</smiles>

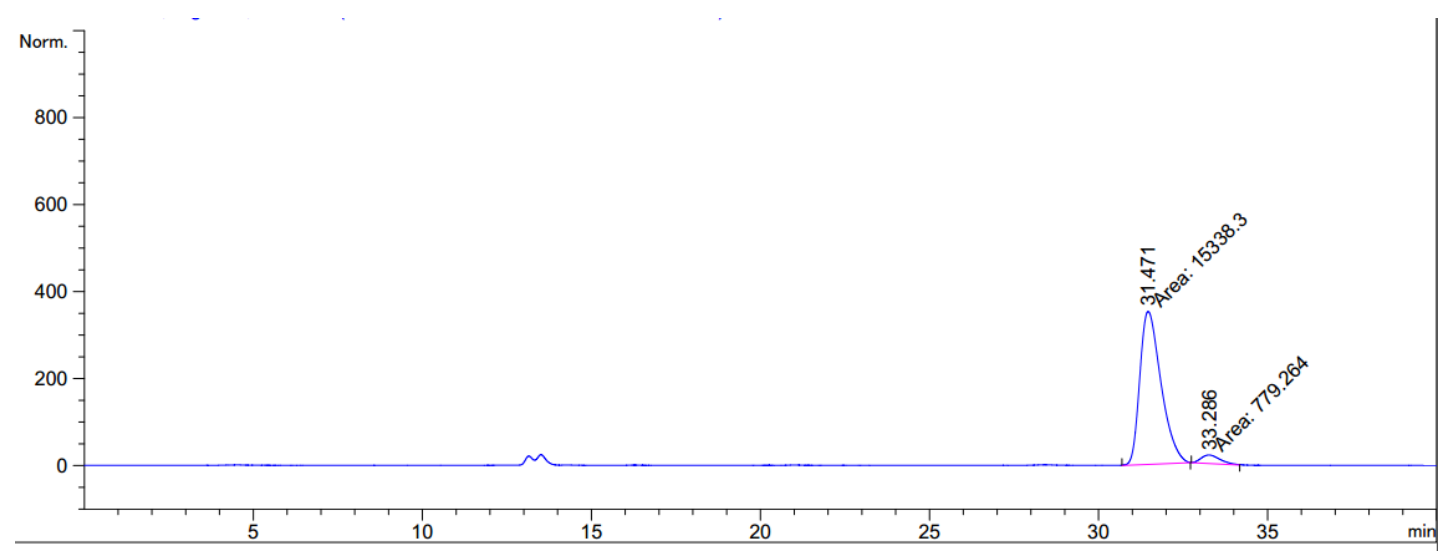

Signal 1: DAD1 E, Sig=280,4 Ref=off

\begin{tabular}{|c|c|c|c|c|c|c|}
\hline $\begin{array}{c}\text { Peak } \\
\quad \#\end{array}$ & $\begin{array}{c}\text { RetTime } \\
\text { [min] }\end{array}$ & Type & $\begin{array}{l}\text { Width } \\
{[\text { min] }}\end{array}$ & $\begin{array}{c}\text { Area } \\
{\left[\mathrm{mAU}^{\star} \mathrm{S}\right]}\end{array}$ & $\begin{array}{l}\text { Height } \\
\text { [mAU] }\end{array}$ & $\begin{array}{c}\text { Area } \\
\frac{\%}{0}\end{array}$ \\
\hline & & & & & & \\
\hline $\begin{array}{l}1 \\
2\end{array}$ & $\begin{array}{l}31.471 \\
33.286\end{array}$ & $\begin{array}{l}\text { MM } \\
\text { MM }\end{array}$ & $\begin{array}{l}0.7265 \\
0.6592\end{array}$ & $\begin{array}{l}1.53383 e 4 \\
779.26410\end{array}$ & $\begin{array}{r}351.89407 \\
19.70347\end{array}$ & $\begin{array}{r}95.1651 \\
4.8349\end{array}$ \\
\hline
\end{tabular}


<smiles>CC(C)=CC=CC(C)O</smiles>

racemate for $\mathbf{2 p}$

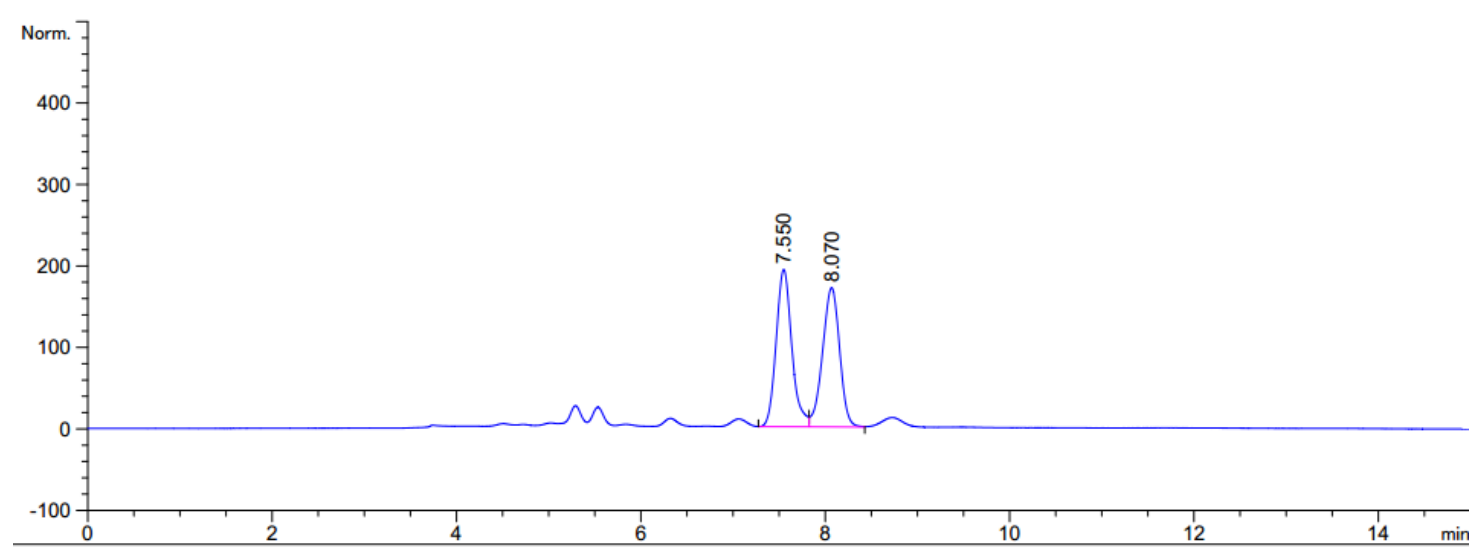

Signal 1: DAD1 B, Sig=254, 4 Ref=off

\begin{tabular}{|c|c|c|c|c|c|c|}
\hline $\begin{array}{c}\text { Peak } \\
\#\end{array}$ & $\begin{array}{c}\text { RetTime } \\
\text { [min] }\end{array}$ & Type & $\begin{array}{c}\text { Width } \\
\text { [min] }\end{array}$ & $\begin{array}{c}\text { Area } \\
{\left[\mathrm{mAU}{ }^{\star} \mathrm{s}\right]}\end{array}$ & $\begin{array}{l}\text { Height } \\
{[\mathrm{mAU}]}\end{array}$ & $\begin{array}{c}\text { Area } \\
\frac{8}{6}\end{array}$ \\
\hline-0 & 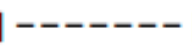 & & - & ---------- & - & --- \\
\hline 1 & 7.550 & BV & 0.1760 & 2282.42969 & 192.96524 & 50.875 \\
\hline 2 & 8.070 & VV & 0.1909 & 2203.87036 & 170.98810 & 49.1245 \\
\hline
\end{tabular}<smiles>CC(C)=C/C=C/[C@H](C)O</smiles>

$2 p$

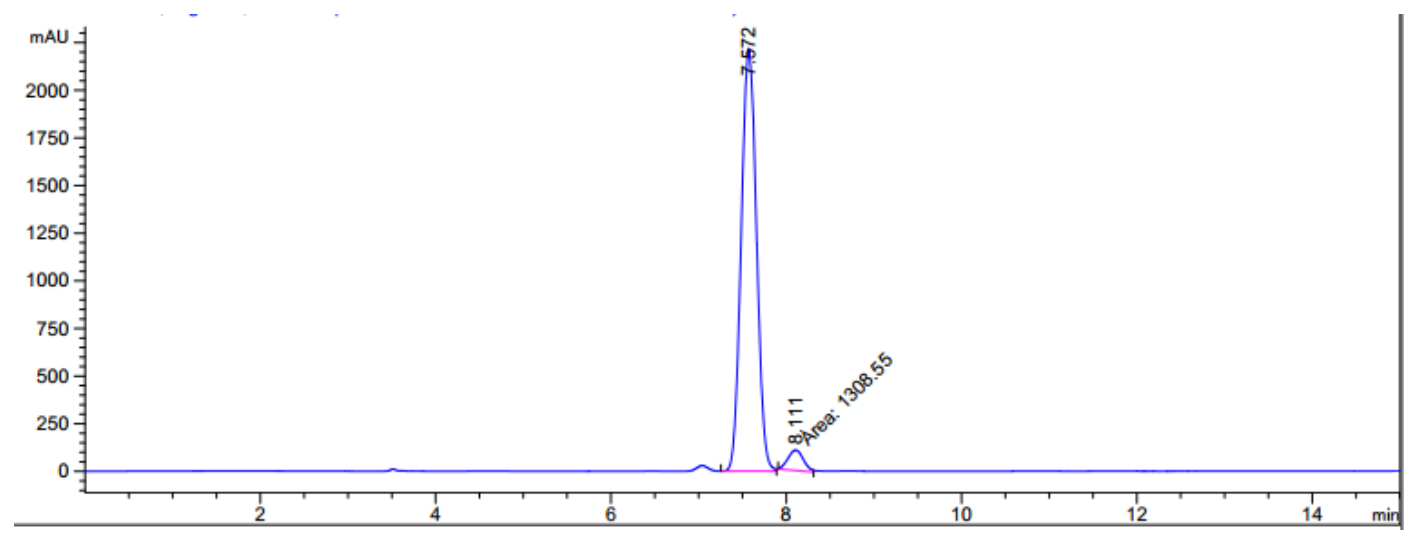

Signal 1: DAD1 B, Sig=254,4 Ref=off

\begin{tabular}{|c|c|c|c|c|c|c|}
\hline $\begin{array}{c}\text { Peak } \\
\#\end{array}$ & $\begin{array}{c}\text { RetTime } \\
\text { [min] }\end{array}$ & Type & $\begin{array}{c}\text { Width } \\
\text { [min] }\end{array}$ & $\begin{array}{c}\text { Area } \\
{\left[\mathrm{mAU}^{\star} \mathrm{S}\right]}\end{array}$ & $\begin{array}{l}\text { Height } \\
\text { [mAU] }\end{array}$ & $\begin{array}{c}\text { Area } \\
\text { 옿 }\end{array}$ \\
\hline- & ------ & & & --------- & |-------- & $-------\mid$ \\
\hline 1 & 7.572 & BV & 0.1431 & $2.68385 e 4$ & 2218.52661 & 95.3510 \\
\hline 2 & 8.111 & MM & 0.2035 & 1308.54895 & 107.16475 & 4.6490 \\
\hline
\end{tabular}


<smiles>C/C=C/C=C/C(C)O</smiles>

racemate for $\mathbf{2 q}$

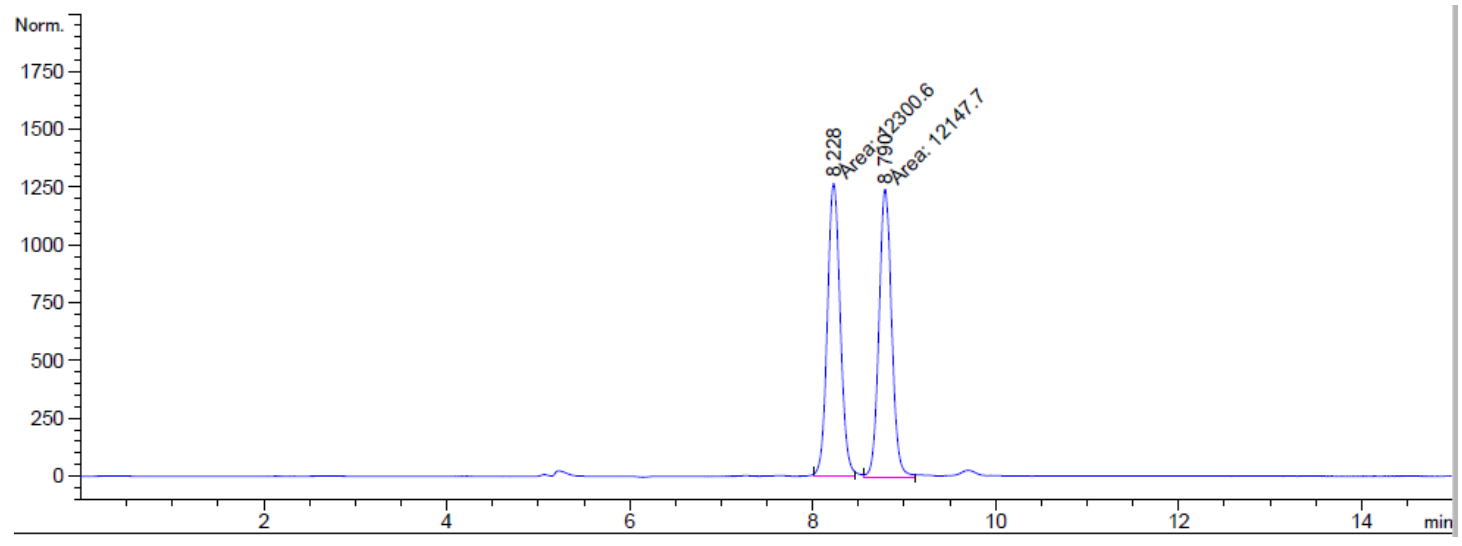

Signal 1: DAD1 C, Sig=210,4 Ref=off

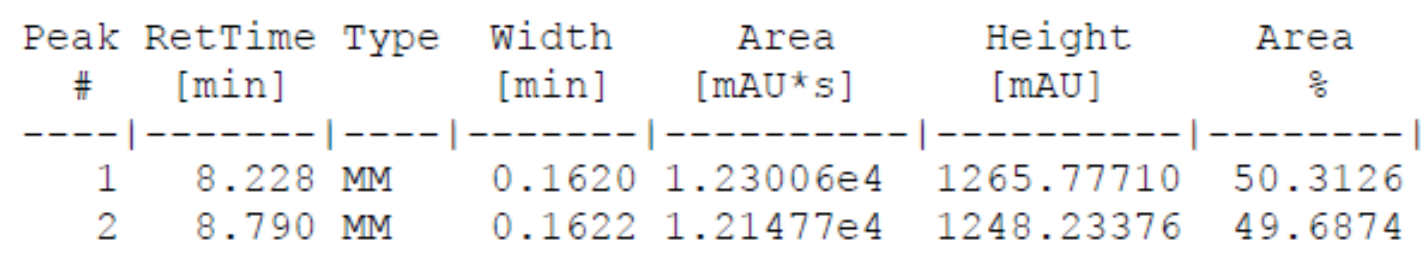<smiles>C/C=C/C=C/C(C)O</smiles>

$2 q$

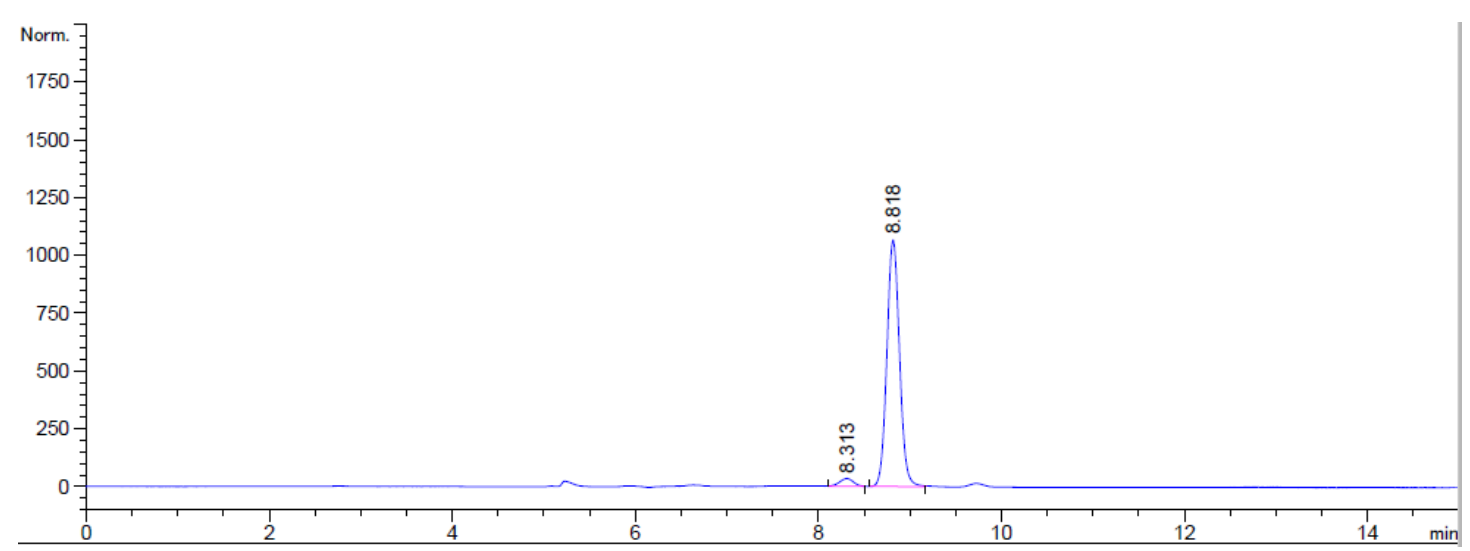

Signal 1: DAD1 C, Sig=210,4 Ref=off

\begin{tabular}{|c|c|c|c|c|c|c|}
\hline $\begin{array}{c}\text { Peak } \\
\#\end{array}$ & $\begin{array}{c}\text { RetTime } \\
\text { [min] }\end{array}$ & Type & $\begin{array}{l}\text { width } \\
\text { [min] }\end{array}$ & $\begin{array}{c}\text { Area } \\
{\left[m A U^{\star} S^{\prime}\right]}\end{array}$ & $\begin{array}{l}\text { Height } \\
{[\mathrm{mAU}]}\end{array}$ & $\begin{array}{c}\text { Area } \\
\%\end{array}$ \\
\hline & & & & $---------\mid$ & --------- & -- \\
\hline $\begin{array}{l}1 \\
2\end{array}$ & $\begin{array}{l}8.313 \\
8.818\end{array}$ & $\begin{array}{l}\text { VV } \\
\text { BV }\end{array}$ & $\begin{array}{l}0.1226 \\
0.1244\end{array}$ & $\begin{array}{c}353.15842 \\
1.01860 e 4\end{array}$ & $\begin{array}{r}34.16260 \\
1066.27808\end{array}$ & $\begin{array}{l}509 \\
491\end{array}$ \\
\hline
\end{tabular}


<smiles>CC(O)/C=C/C=C/c1ccco1</smiles>

racemate for $2 r$

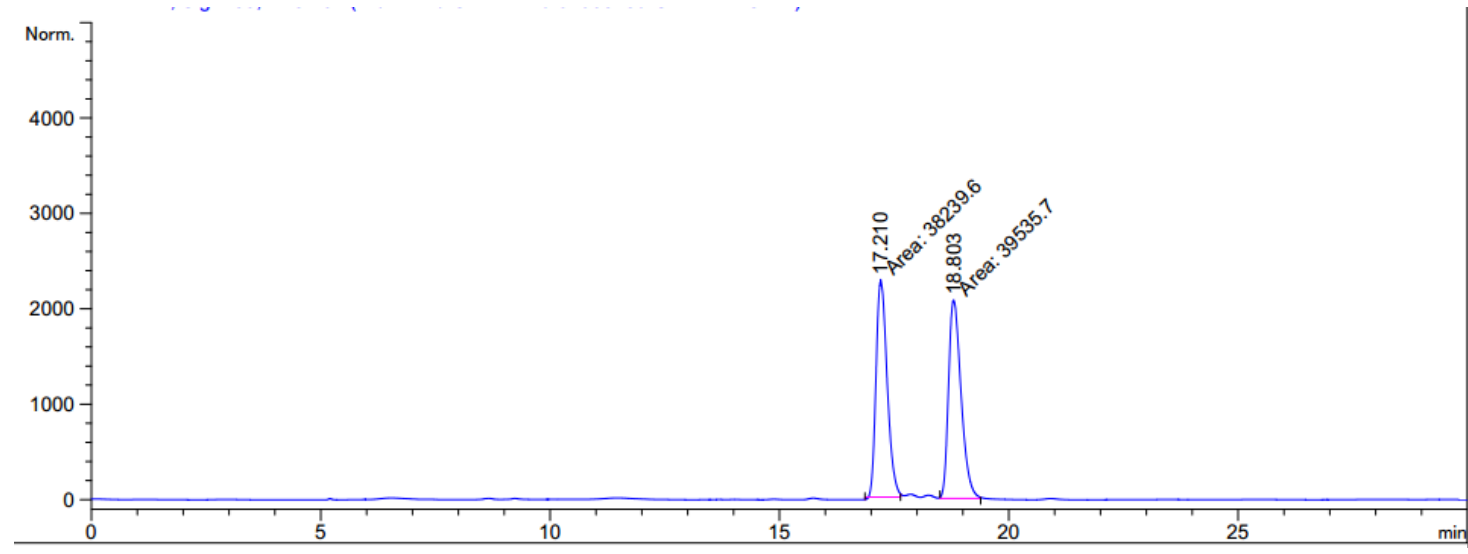

Signal 1: DAD1 E, Sig=280,4 Ref=off

\begin{tabular}{|c|c|c|c|c|c|c|}
\hline $\begin{array}{c}\text { Peak } \\
\quad \#\end{array}$ & $\begin{array}{c}\text { RetTime } \\
\text { [min] }\end{array}$ & Type & $\begin{array}{l}\text { Width } \\
\text { [min] }\end{array}$ & $\begin{array}{c}\text { Area } \\
{\left[\mathrm{mAU}{ }^{\star} \mathrm{s}\right]}\end{array}$ & $\begin{array}{l}\text { Height } \\
\text { [mAU] }\end{array}$ & $\begin{array}{c}\text { Area } \\
\frac{8}{\partial}\end{array}$ \\
\hline 1 & 1 & $\mathbb{M}$ & & $82396 \mathrm{e} 4$ & 2279.90381 & 668 \\
\hline 2 & 18.803 & MM & 0.3168 & $3.95357 \mathrm{e} 4$ & 2079.69360 & 50.8332 \\
\hline
\end{tabular}<smiles>C[C@H](O)/C=C/C=C/c1ccco1</smiles>

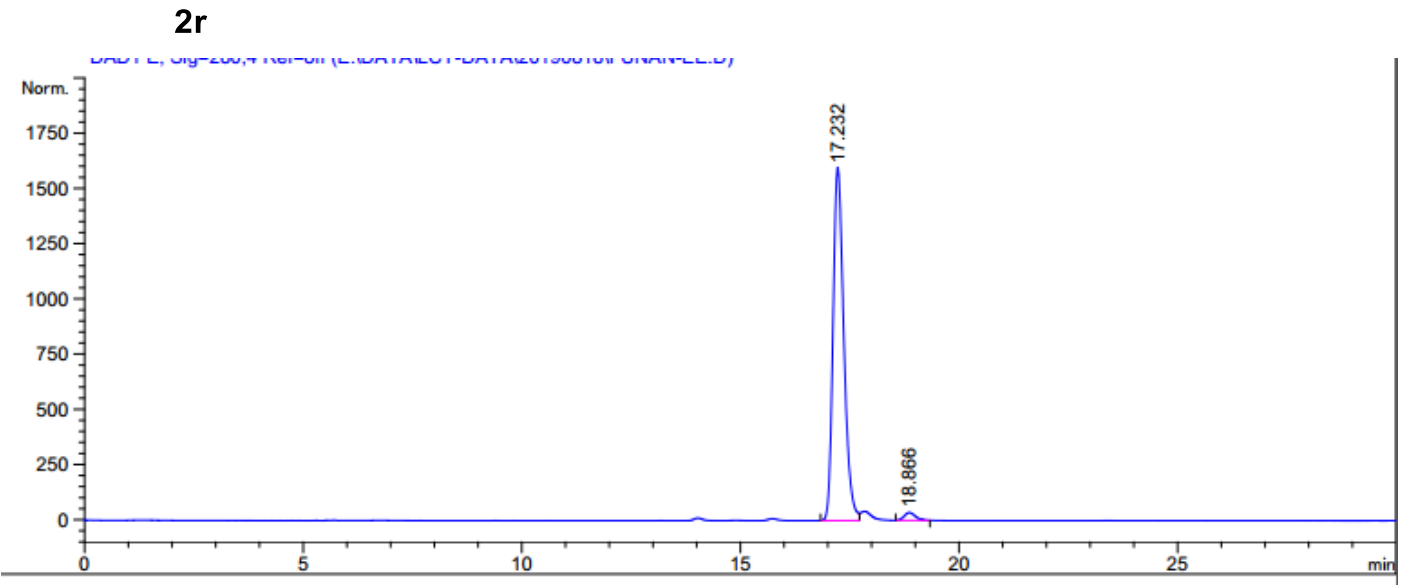

Signal 1: DAD1 E, Sig=280, 4 Ref $=$ off

\begin{tabular}{|c|c|c|c|c|c|c|}
\hline $\begin{array}{c}\text { Peak } \\
\#\end{array}$ & $\begin{array}{c}\text { RetTime } \\
\text { [min] }\end{array}$ & Type & $\begin{array}{l}\text { Width } \\
\text { [min] }\end{array}$ & $\begin{array}{c}\text { Area } \\
{\left[\mathrm{mAU}^{*} \mathrm{~s}\right]}\end{array}$ & $\begin{array}{l}\text { Height } \\
\text { [mAU] }\end{array}$ & $\begin{array}{c}\text { Area } \\
\frac{8}{6}\end{array}$ \\
\hline$=-$ & $-0-0$ & & - & $-----0--0-$ & 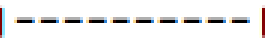 & -----1 \\
\hline 1 & 17.232 & vV & 0.2072 & $2.73673 \mathrm{e} 4$ & 1595.01526 & 97.7220 \\
\hline 2 & 18.866 & VV & 0.2095 & 637.96539 & 35.77692 & 2.2780 \\
\hline
\end{tabular}



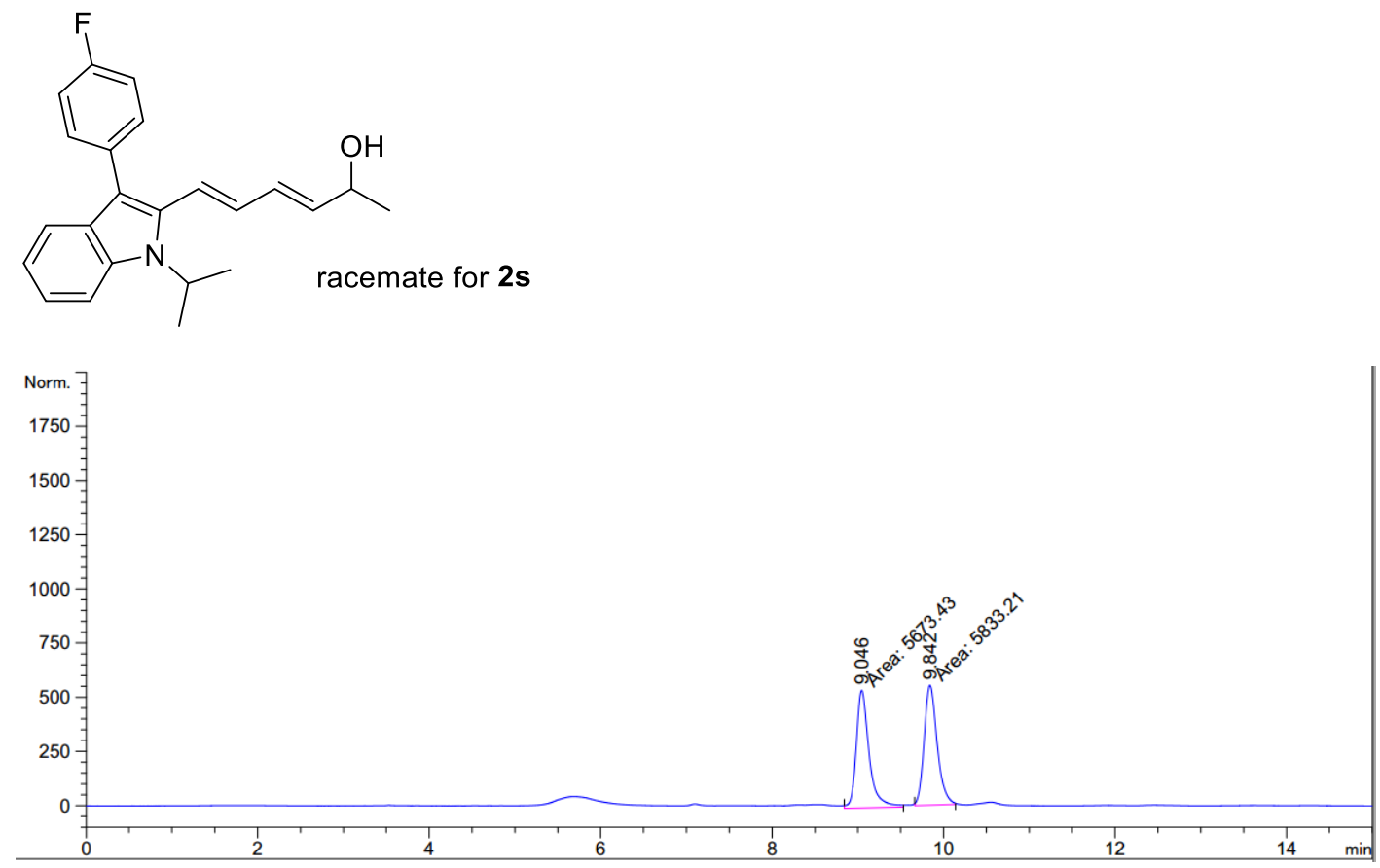

Signal 1: DAD1 E, Sig=280,4 Ref=off
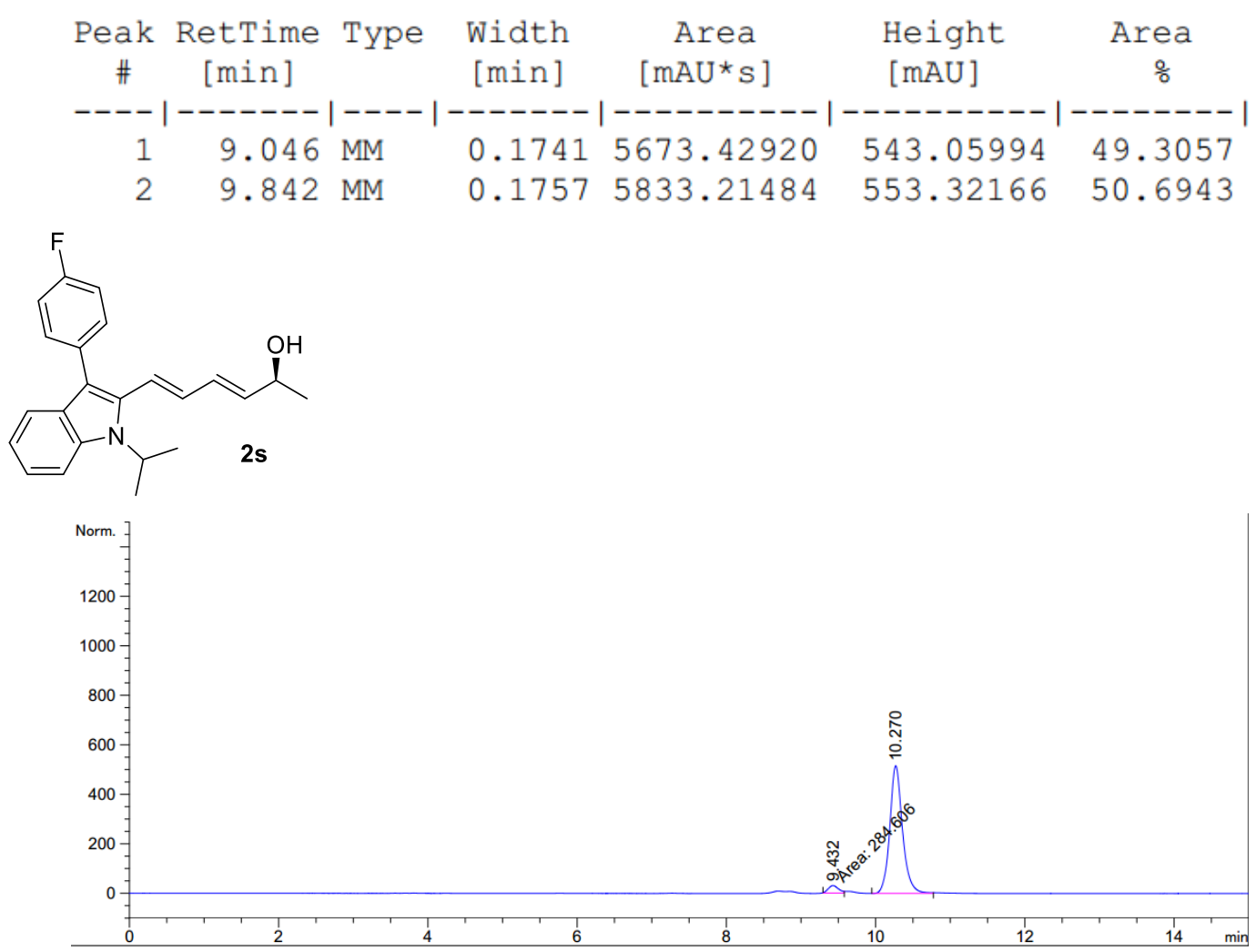

Signal 1: DAD1 E, Sig=280,4 Ref=off

\begin{tabular}{|c|c|c|c|c|c|c|}
\hline $\begin{array}{c}\text { eak } \\
\#\end{array}$ & $\begin{array}{l}\text { RetTime } \\
\text { [min] }\end{array}$ & Type & $\begin{array}{l}\text { Width } \\
\text { [min] }\end{array}$ & $\begin{array}{c}\text { Area } \\
{\left[\mathrm{mAU} U^{\star} \mathrm{S}\right]}\end{array}$ & $\begin{array}{l}\text { Height } \\
\text { [mAU] }\end{array}$ & $\begin{array}{c}\text { Area } \\
\frac{\circ}{0}\end{array}$ \\
\hline & & & & & & \\
\hline
\end{tabular}


<smiles>OC(/C=C/C=C/c1ccccc1)c1ccccc1</smiles>

racemate for $\mathbf{2 t}$

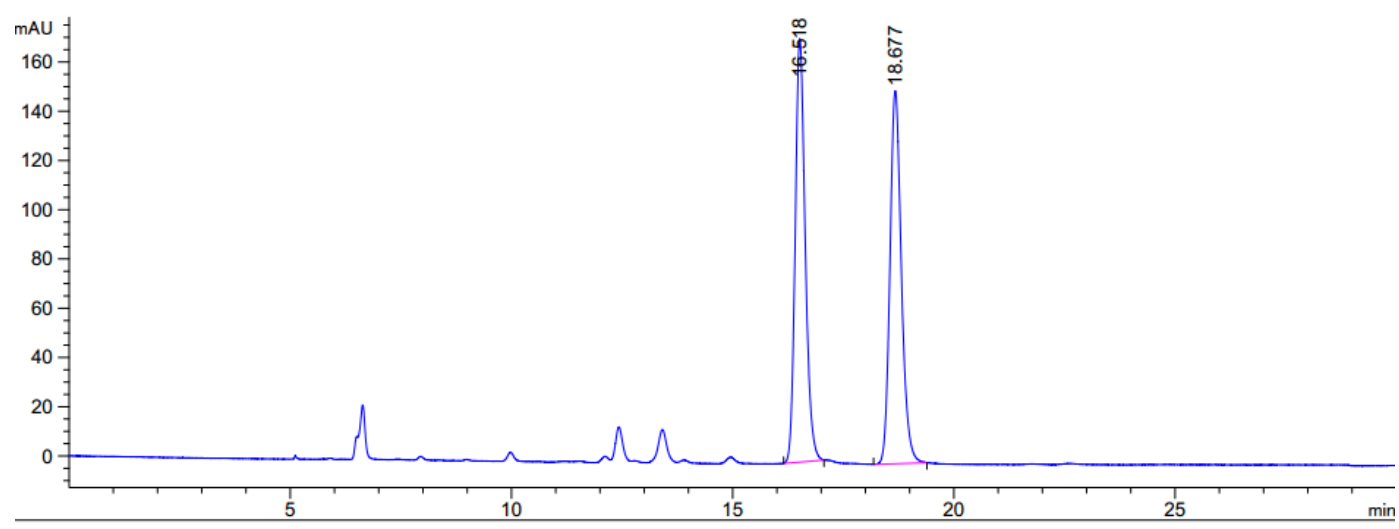

Signal 1: DAD1 B, Sig=254,4 Ref=off

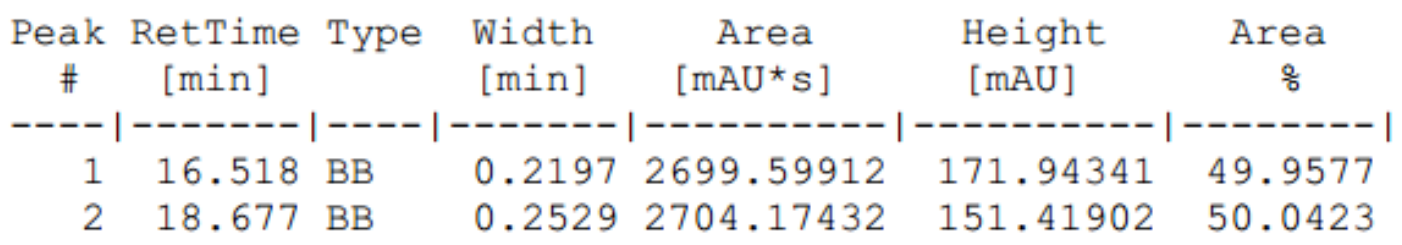<smiles>OC(/C=C/C=C/c1ccccc1)c1ccccc1</smiles>

2t

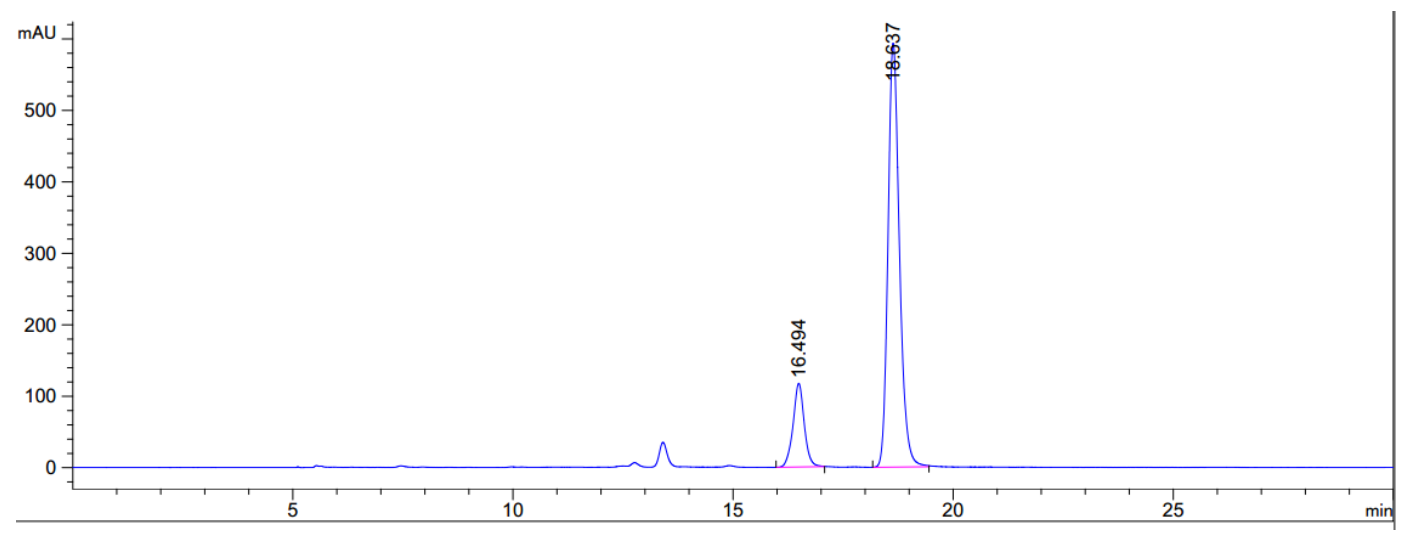

Signal 1: DAD1 B, Sig=254,4 Ref=off

\begin{tabular}{|c|c|c|c|c|c|c|}
\hline $\begin{array}{c}\text { Peak } \\
\#\end{array}$ & $\begin{array}{c}\text { RetTime } \\
\text { [min] }\end{array}$ & Type & $\begin{array}{c}\text { Width } \\
\text { [min] }\end{array}$ & $\begin{array}{c}\text { Area } \\
{\left[\mathrm{mAU}^{*} \mathrm{~S}\right]}\end{array}$ & $\begin{array}{l}\text { Height } \\
\text { [mAU] }\end{array}$ & $\begin{array}{c}\text { Area } \\
\frac{\%}{\delta}\end{array}$ \\
\hline-- & 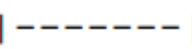 & & ----- & -------- & -------- & ---1 \\
\hline 1 & 16.494 & BV & 0.2300 & 2073.53101 & 117.14567 & 16.2360 \\
\hline 2 & 18.637 & BV & 0.2678 & $1.06976 \mathrm{e} 4$ & 593.48291 & 83.7640 \\
\hline
\end{tabular}


<smiles>OC(/C=C/C=C/c1ccccc1)c1ccccc1Cl</smiles>

racemate for $2 u$

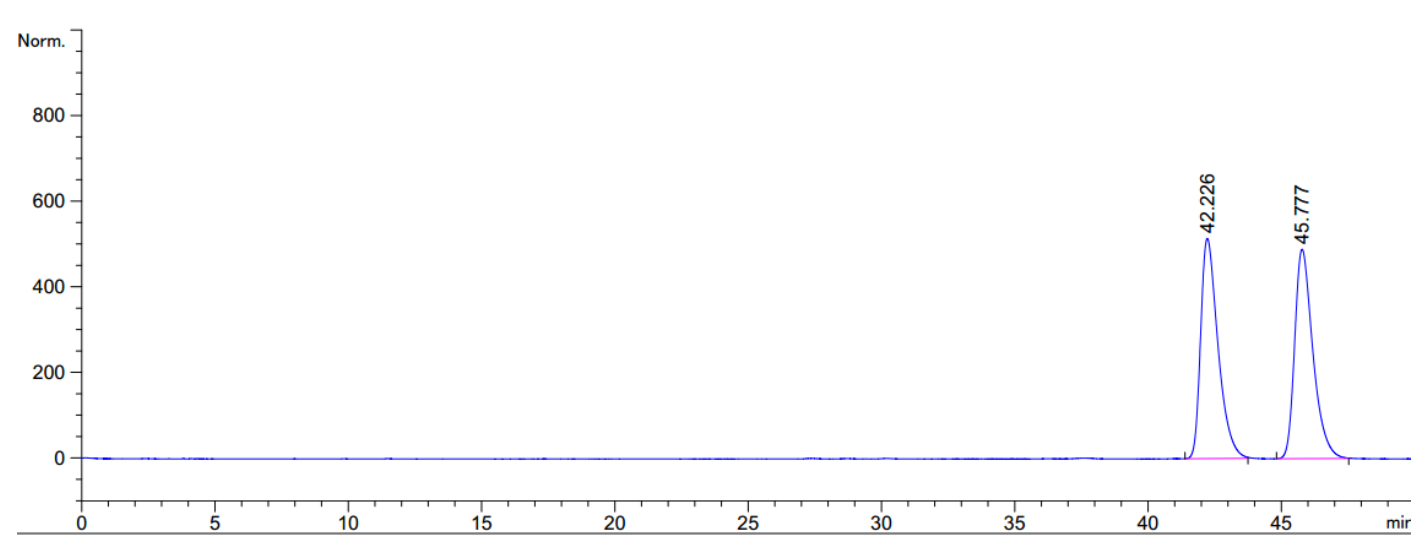

Signal 1: DAD1 E, Sig=280,4 Ref=off

\begin{tabular}{|c|c|c|c|c|c|c|}
\hline $\begin{array}{c}\text { Peak } \\
\quad \#\end{array}$ & $\begin{array}{c}\text { RetTime } \\
\text { [min] }\end{array}$ & Type & $\begin{array}{c}\text { Width } \\
{[\mathrm{min}]}\end{array}$ & $\begin{array}{c}\text { Area } \\
{\left[\mathrm{mAU}^{\star} \mathrm{s}\right]}\end{array}$ & $\begin{array}{l}\text { Height } \\
\text { [mAU] }\end{array}$ & $\begin{array}{c}\text { Area } \\
\%\end{array}$ \\
\hline$=--$ & 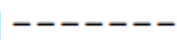 & & & ---- & 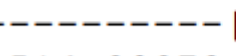 & -------- \\
\hline 1 & 42.226 & VV & 0.5278 & $2.32246 \mathrm{e} 4$ & 514.80872 & 50.0024 \\
\hline 2 & 45.777 & VV & 0.5566 & $2.32224 \mathrm{e} 4$ & 489.37970 & 49.9976 \\
\hline
\end{tabular}<smiles>OC(/C=C/C=C/c1ccccc1)c1ccccc1Cl</smiles>

$2 u$

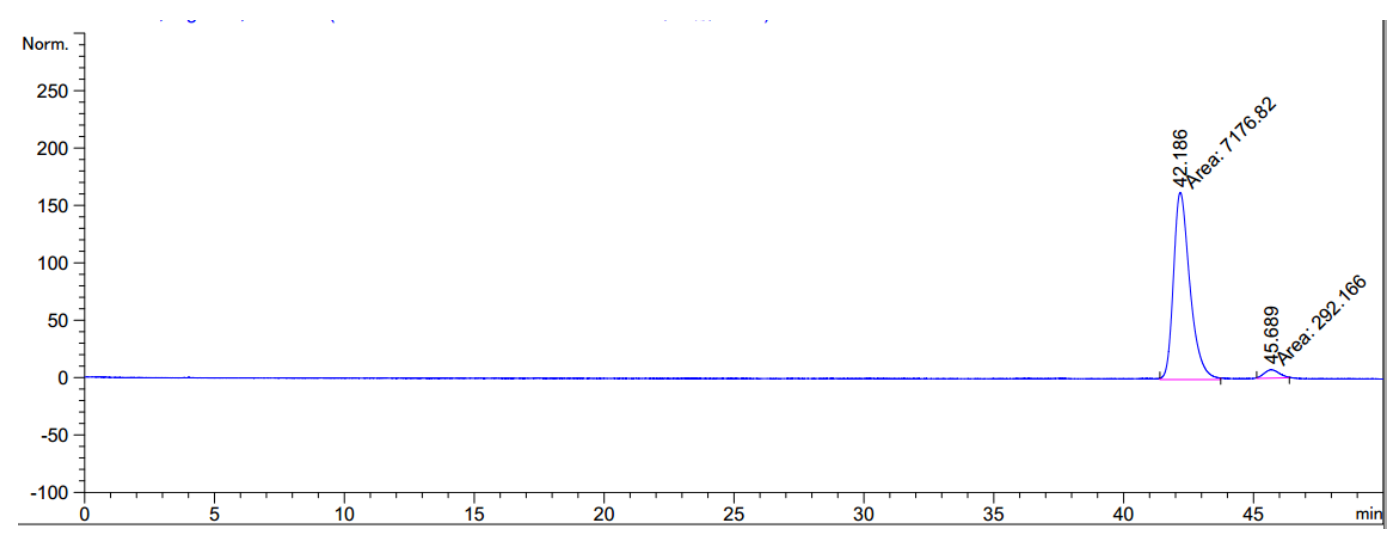

Signal 1: DAD1 E, Sig=280,4 Ref=off

\begin{tabular}{|c|c|c|c|c|c|c|}
\hline $\begin{array}{c}\text { Peak } \\
\quad \#\end{array}$ & $\begin{array}{l}\text { RetTime } \\
\text { [min] }\end{array}$ & Type & $\begin{array}{c}\text { Width } \\
\text { [min] }\end{array}$ & $\begin{array}{c}\text { Area } \\
{\left[\mathrm{mAU}^{\star} \mathrm{s}\right]}\end{array}$ & $\begin{array}{l}\text { Height } \\
\text { [mAU] }\end{array}$ & $\begin{array}{c}\text { Area } \\
\frac{\circ}{0}\end{array}$ \\
\hline 1 & 42.186 & MM & 0.7331 & 7176.82129 & 163.16011 & 96.0883 \\
\hline 2 & 45.689 & MM & 0.6695 & 292.16583 & 7.27352 & 3.9117 \\
\hline
\end{tabular}

